\title{
Local and Global Analysis of Relaxed DOUGLas-RACHFORD FOR
}

Nonconvex FeAsibility Problems

\author{
Dissertation \\ zur Erlangung des mathematisch-naturwissenschaftlichen Doktorgrades \\ "Doctor rerum naturalium" \\ der Georg-August-Universität Göttingen
}

im Promotionsprogramm Mathematical Sciences

der Georg-August University School of Science (GAUSS)

vorgelegt von

Anna-Lena Martins

aus Buxtehude

Göttingen, 2019 


\section{Betreuungsausschuss:}

Prof. Dr. Russell Luke

Institut für Numerische und Angewandte Mathematik

Georg-August-Universität Göttingen

Prof. Dr. Thorsten Hohage

Institut für Numerische und Angewandte Mathematik

Georg-August-Universität Göttingen

\section{Mitglieder der Prüfungskommission:}

\section{Referent:}

Prof. Dr. Russell Luke

Institut für Numerische und Angewandte Mathematik

Georg-August-Universität Göttingen

\section{Korreferent:}

Prof. Dr. Thorsten Hohage

Institut für Numerische und Angewandte Mathematik Georg-August-Universität Göttingen

\section{Weitere Mitglieder der Prüfungskommission:}

Prof. Dr. Jörg Brüdern

Mathematisches Institut

Georg-August-Universität Göttingen

Prof. Dr. Stephan Huckemann

Institut für Mathematische Stochastik

Georg-August-Universität Göttingen

Prof. Dr. Gerlind Plonka-Hoch

Institut für Numerische und Angewandte Mathematik Georg-August-Universität Göttingen

Prof. Dr. Anja Sturm

Institut für Mathematische Stochastik

Georg-August-Universität Göttingen

Tag der mündichten Prüfung: 19.03.2019 


\section{ACKNOWLEDGEMENTS}

To my supervisor Russell Luke I wish to express my deepest gratitude for the last three years. Without your guidance and support I would have been lost during this time. Your knowledge and enthusiasm always helped whenever I was in doubt about a problem.

My gratitude also goes to my co-supervisor Thorsten Hohage for his support and helpful comments. I am thankful for the financial support by the SFB 755 and the GRK 2088. Their meetings will always be a good memory.

During the last three (and something) years my working group became a big part of my life. Without you my PhD time would not have been as good as it was. Thank you for coffee, chocolate, beer, wine, and of course all the nice moments we had together. Especially I would like to thank Neal for being such a good officemate. Getting distracted from work was always fun. To Matt, Neal and Yura I am grateful for proofreading this thesis.

For always having a sympathetic ear when I wanted to complain about mathematics I have to express my gratitude to Felix, Kathi and Maxi. Thank you for being such good friends and your valuable comments on this work.

Last but not least I have to thank Jörn. Thank you for your love and always being there. Without you I would not have finished this work!

P.S. To everyone I forgot to mention here: Thank you! 

Abstract $\quad x i$

1 Introduction $\quad 1$

2 Notation and Preliminaries 5

2.1 Feasibility Problems . . . . . . . . . . . . . . . 5

2.2 Projectors and Reflectors . . . . . . . . . . . . . . . . . 6

2.3 Projection Methods . . . . . . . . . . . . . . . . . . . 10

3 A Toolkit for Convergence 15

3.1 Regularities of Mappings . . . . . . . . . . . . . . . . . . . . . . . 15

3.2 Convergence Theorems . . . . . . . . . . . . . . . . . . . 20

3.3 Regularities of Sets . . . . . . . . . . . . . . . . . . . 23

3.4 Regularities of Collections of Sets . . . . . . . . . . . . 30

4 Relaxed Douglas-Rachford $\quad 35$

$4.1 T_{A, B}^{\lambda}$ is Almost Averaged at Fix $T_{A, B}^{\lambda} \ldots \ldots \ldots . \ldots . \ldots 36$

4.2 Characterization of Fix $T_{A, B}^{\lambda} \ldots \ldots \ldots \ldots$. . . . . . . . 41

$4.3 T_{A, B}^{\lambda}$ at Fix $T_{A, B}^{\lambda}$ : Metric Subregularity . . . . . . . . . . . . . . 49

4.4 Local Linear Convergence of $T_{A, B}^{\lambda} \ldots \ldots \ldots \ldots$

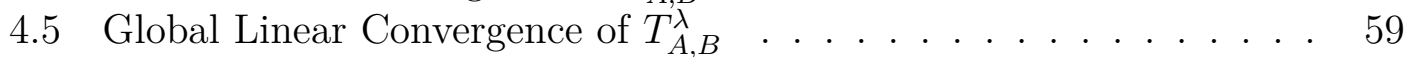

4.6 Elementary Examples . . . . . . . . . . . . . . . . . . 62

4.7 Special Case: Subspaces . . . . . . . . . . . . . . . . . 72

5 Cyclic Relaxed Douglas-Rachford $\quad 83$

5.1 General Convergence Analysis . . . . . . . . . . . . . . . . . . . 84

5.2 Fixed Points for Two Convex Sets . . . . . . . . . . . . . . . . . 86

5.3 Fixed Points for $m$ Subspaces . . . . . . . . . . . . . . . . . . . 91

6 Phase Retrieval $\quad 95$

6.1 Phase Retrieval as a Feasibility Problem . . . . . . . . . . . . . . 96

6.2 Projectors onto Constraint Sets . . . . . . . . . . . . . . . . . . . 99 
6.3 Regularities of Constraint Sets . . . . . . . . . . . . . . . . . . . 101

6.4 Phase Retrieval Algorithms . . . . . . . . . . . . . . . . . . . . . 102

6.5 Convergence Results . . . . . . . . . . . . . . . . . . . . . . . 104

6.6 Numerical Analysis . . . . . . . . . . . . . . . . . . . 106

$\begin{array}{ll}7 \text { A Matrix World Approach } & 115\end{array}$

8 Conclusion and Future Work 123

8.1 Relaxed Douglas-Rachford . . . . . . . . . . . . . . . . . . . . 124

8.2 Cyclic Relaxed Douglas-Rachford . . . . . . . . . . . . . . . . . . . 124

8.3 Phase Retrieval . . . . . . . . . . . . . . . . . . . 125

$\begin{array}{lr}\text { Bibliography } & 127\end{array}$

$\begin{array}{ll}\text { Index } & 137\end{array}$

$\begin{array}{ll}\text { Notation and Symbols } & 139\end{array}$

$\begin{array}{ll}\text { Curriculum Vitae } & 141\end{array}$ 


\section{List OF FiguRES}

2.1 Examples of projectors and reflectors. . . . . . . . . . . .

2.2 Illustration of MAP, DR, relaxed DR and its cyclic version $(\lambda=0.4)$ applied to two affine sets $A=\mathbb{R} \times\{0\}$ and $B=\left\{x \in \mathbb{R}^{2} \mid x_{1}=x_{2}\right\}$ with initial point $x=(1,2) \in \mathbb{R}^{2}$ showing 5 iterations. . . . . . . 12

3.1 Illustration of the sets in Example 3.1.1. . . . . . . . . . . . . 16

3.2 Illustration of the sets in Example 3.3.8. . . . . . . . . . . . . 25

3.3 Illustration of the sets in Example 3.3.10. . . . . . . . . . . . . . 27

4.1 Illustration of applying $T_{A, B}^{\lambda}$ on a point $x$ yielding $y=T_{A, B}^{\lambda} x$ for a

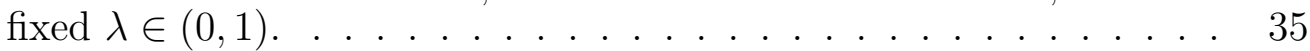

4.2 Illustration of a possible choice of neighborhoods in Example 4.1.4(ii). 40

4.3 Illustration of the sets in Example 4.1.4(i)-(iii). . . . . . . . . . . . 41

4.4 Illustration of the sets $E$ and $F$ and the displacement vector $g$ in the three different settings of Example 4.2.5 . . . . . . . . . . 45

4.5 Example 4.2 .10 for a point $x \in \mathbb{R}^{2}$ and $\lambda=0.8$. . . . . . . . . . 47

4.6 Framework for the convergence analysis illustrated in $\mathcal{E}$. . . . . . . 50

4.7 Illustration of the sets in Example 4.6.1 for $a=-1.5$ and $R=1$. . . 63

4.8 Illustration of the sets in Example 4.6.2 for $R=1 \ldots$. . . . . . . 67

4.9 Illustration of the sets in Example 4.6.3 for $R=1 \ldots \ldots$. . . . . . 68

4.10 Illustration of the sets in Example 4.6 .4 for $R=2$. . . . . . . . . . 70

4.11 Illustration of the sets in Example 4.6.5 for $R=1$. . . . . . . . . . 70

4.12 Illustration of the sets $A$ and $B$ in Example 4.7.15. . . . . . . . . 80

5.1 Illustration of cyclic relaxed DR $(\lambda=0.4)$ applied to two affine sets $A=\mathbb{R} \times\{0\}$ and $B=\left\{x \in \mathbb{R}^{2} \mid x_{1}=x_{2}\right\}$ and applied to two balls $A=\left\{x \in \mathbb{R}^{2} \mid x_{1}^{2}+x_{2}^{2} \leq 1\right\}$ and $B=\left\{x \in \mathbb{R}^{2} \mid\left(x_{1}^{2}-4\right)+x^{2} \leq 4\right\}$ with initial point $x=(1,2) \in \mathbb{R}^{2}$ showing 4 iterations each. Each arrow represents a 2-set relaxed Douglas-Rachford iteration. . . . . 87

5.2 Illustration of Fix $\left.T_{[A}^{\lambda} B\right]$ for two closed convex sets $A$ and $B(\lambda=0.4) .90$

5.3 Cyclic relaxed DR $(\lambda=0.4)$ applied to three lines in $\mathbb{R}^{2}$. Shown are the iterations and the fixed points for cyclic projections $(A, B, C)$ for some initial point $x^{0}$. Each green line represents a 2 -set relaxed Douglas-Rachford iteration. . . . . . . . . . . . . . . . 
5.4 Cyclic relaxed DR $(\lambda=0.4)$ applied to three balls in $\mathbb{R}^{2}$. Shown are the iterations and the fixed points for cyclic projections $(D, E, F)$ for some initial point $x^{0}$. Each green line represents a 2-set relaxed Douglas-Rachford iteration. . . . . . . . . . . . . . . . . 94

6.1 Measurement and support constraint. . . . . . . . . . . . . . . 107

6.2 Change in iterates for data set "tasse" until iteration 1000. . . . . . 108

6.3 Change in iterates for full data set "tasse". . . . . . . . . . . . . . 108

6.4 Gap for data set "tasse" until iteration 50. . . . . . . . . . . . . . . 109

6.5 Change in iterates for limited data set ("tasse"). . . . . . . . . . . . 110

6.6 Gap for limited data set ("tasse") until iteration 50. . . . . . . . . . 110

6.7 Reconstruction of the optical diffraction experiment "tasse" using MAP, relaxed DR and its cyclic version. . . . . . . . . . . . . 112 


\section{LIST OF TABLES}

6.1 Comparison of the number of iterations till stopping criterium is reached of MAP, relaxed DR and its cyclic version. . . . . . . . . . 111

6.2 Mean, minimum, and maximum number of iterations of MAP, relaxed DR and its cyclic version for 100 random instances. . . . . . . 111

6.3 Mean, minimum, and maximum number of iterations of $\mathrm{CP}$, relaxed DR and its cyclic version for 100 random instances on synthetic data.113

6.4 Mean, minimum, and maximum relative error to the true solution $\times 10^{-} 5$ of $\mathrm{CP}$, relaxed $\mathrm{DR}$ and its cyclic version for 100 random instances on synthetic data. . . . . . . . . . . . . . . 113 



\section{AbstraCt}

This thesis investigates the local and global convergence analysis of the relaxed Douglas-Rachford method. This algorithm, which was first proposed over a decade ago, has become a standard procedure in applications. Convergence results for this algorithm are limited either to convex feasibility or consistent nonconvex feasibility with strong assumptions on the regularity of the underlying sets. After discussing feasibility problems and projection methods to solve these in general, we investigate the relaxed Douglas-Rachford method in detail for inconsistent and nonconvex feasibility problems. By introducing a new type of regularity of sets, called superregularity at a distance, we establish sufficient conditions for local linear convergence of the corresponding sequence for the method of relaxed Douglas-Rachford subsuming already existing results in the literature. We analyze a cyclic relaxed Douglas-Rachford scheme and state convergence results for closed and convex sets, by considering many-set feasibility problems. We then apply the theory developed to the famous phase retrieval problem and discuss the numerical performance of the algorithms. 



\section{CHAPTER 1}

\section{INTRODUCTION}

Feasibility problems can be found in numerous areas such as engineering, physics and economics. Given constraints that describe the problem instance, one is seeking a solution that fits all of the constraints. In physics, this framework can represent some experiment including the experimental setup and some measurements. Problems in economics might consist of empirical observations and some theoretical model. In mathematics, we can describe the constraints in each of these problem instances as sets of points in a suitable space. A solution to the problem is then a point that lies in all of the constraint sets. Thus, a feasibility problem consists in finding a point in the collection of a finite number of sets as introduced in Section 2.1. If no such point exists, that is, the sets do not intersect, we are instead seeking an adequate approximation to the problem. We call the problem in this case inconsistent. Feasibility problems are commonly solved by projection methods. These are iterative algorithms defined by a mapping $T$, composed of projectors, that generate a sequence $\left(x^{k}\right)_{k \in \mathbb{N}}$ by

$$
x^{k+1} \in T x^{k} .
$$

Some of the most famous projection algorithms are von Neumann's method of alternating projections for two sets [124], and its many set version the cyclic projection algorithm first discovered by Kaczmarz [83]. A different projection algorithm for a two set approach is the Douglas-Rachford algorithm [56]. Due to their simplicity alternating projections and its cyclic version enjoy great popularity. Although the iterates of these procedures almost always converge, the corresponding limit might have no connection to the initial feasibility problem (see for instance [7]). While the Douglas-Rachford method shows remarkable performances in feasibility problems when both sets intersect, it diverges when the problem is inconsistent. By presenting theoretical tools to prove convergence of general projection methods, we focus in this thesis on a particular algorithm for two sets problems avoiding the drawbacks of the cyclic projections algorithm and the Douglas-Rachford algorithm just mentioned: the relaxed Douglas-Rachford method. Formulated over a decade ago by Luke [97], this projection method is defined by a mapping that is a convex 
composition of the Douglas-Rachford method and a single projection. In fact, given two sets $A$ and $B$ and some initial point $x^{0}$ the relaxed Douglas-Rachford method generates a sequence $\left(x^{k}\right)_{k \in \mathbb{N}}$ by

$$
x^{k+1} \in T_{A, B}^{\lambda} x^{k}:=\bigcup_{b \in P_{B} x^{k}}\left\{\frac{\lambda}{2}\left(R_{A}\left(2 b-x^{k}\right)+x^{k}\right)+(1-\lambda) b\right\} \quad(\forall k \in \mathbb{N}),
$$

where the first part in this definition, $\frac{1}{2}\left(R_{A}\left(2 b-x^{k}\right)+x^{k}\right)$, is a Douglas-Rachford step. Introducing a new type of regularity, super-regularity at a distance, we are able to prove local convergence of the algorithm for nonconvex inconsistent feasibility problems. Our results subsume previous works that depend on strong regularity assumptions like convexity (see $[97,98]$ ), or show only convergence to stationary points (see for instance [94]). To do so, we rely on a framework established by Luke, Thao and Tam in [105]. In addition to the existence of fixed points of the related mapping, here $T_{A, B}^{\lambda}$, there are two main ingredients in the local convergence recipe. The first is pointwise almost averagedness of the mapping. In the context of projection methods and feasibility problems, averagedness of the mapping is strongly connected to the regularity properties of the involved sets (see Section 3.3). The notion of super-regularity at a distance allows us to describe the regularity of a set relative to a point not in the set and is a special case of other, more general, regularity notions like $\epsilon$-subregularity (see $[48,105]$ ). This is of particular importance when analyzing convergence of the algorithms to fixed points that are not in either of the two sets (see Section 4.2). The second ingredient is metric subregularity of a related mapping, $T_{A, B}^{\lambda}-\mathrm{Id}$, a property that was recently shown to be necessary for local linear convergence [104]. While averagedness is dependent on the regularity of the sets itself, metric subregularity relies on the regularity of the collection of sets. In the context of this thesis we work with substransversality. The authors in [104] used this notion to cover the regularity of an inconsistent collection of sets. If we are in the setting of consistent feasibility subtransversality was shown to be necessary for R-linear convergence of alternating projections [104]. If, in addition, the sets are convex, subtransversality is even necessary and sufficient, i.e. equivalent to R-linear convergence [104, Theorem 8 and 9]. In Chapter 4, we present a description of the fixed points of the relaxed Douglas-Rachford method for super-regular at a distance sets. Moreover, we demonstrate in the main result of Section 4.4 how the assumptions on the regularities of both the sets $A$ and $B$ as well as their collection $\{A, B\}$ influence the local convergence behavior of the algorithm independent of whether or not the sets $A$ and $B$ have points in common. Restricting both sets to be convex, we can even prove global convergence. In Section 4.7, we present a class of sets that always satisfy the assumptions of the local convergence result globally. In fact, we investigate subspaces and compare our results on the relaxed Douglas-Rachford method to other projection methods using the notion of the Friedrichs angle to express the regularity of the collection of sets.

The downside of the relaxed Douglas-Rachford method is its formulation for just two sets. To overcome this, we propose the cyclic relaxed Douglas-Rachford method 
in Chapter 5. First mentioned but not theoretically discussed in [103] by Luke, Sabach and Teboulle, this algorithm is designed for many-set feasibility problems. The authors in [103] analyzed the numerical performance of this algorithm, justifying a deeper theoretical analysis. As a first step, we analyze its behavior when applied to convex sets and show convergence as well as an explicit description of the set of fixed points for the 2-set case that differs from the original relaxed DouglasRachford method. Moreover, we discuss the many-set case on subspaces. Parts of these results were published in [102] in co-authorship with Matthew K. Tam and D. Russell Luke.

In addition to the general discussion of feasibility problems and our approach for the relaxed Douglas-Rachford method and its cyclic version, we focus on a specific feasibility problem, the phase retrieval problem. This problem is known from the field of physics and appears there in several areas such as microscopy, astronomy, optical design and holography. Given some a priori knowledge about the experimental setup and some measurements, one has to reconstruct the object that was measured. The problem is that the measurements only contain the modulus of the Fourier transform of the object but not the phase. We present popular algorithms to solve this problem and demonstrate how they fit into the framework of feasibility problems in Chapter 6. Using the results from the chapters before, we state local convergence results of the relaxed Douglas-Rachford method and other algorithms for the phase retrieval problem. Comparing several algorithms, we point out characteristics of their individual numerical performance and theoretical features used in the statements given. We expand the discussion of the phase retrieval problem with a chapter on uniqueness for this nonconvex problem, Chapter 7. By analyzing when the phase retrieval problem has a unique solution, we discuss an approach for when fixed points of projection methods are not just approximations but solutions to the problem. We close this thesis with a short conclusion and outline possible branches and questions for further research. 



\section{CHAPTER 2}

\section{Notation AND PRELiminaries}

This chapter introduces the basic notation used in this thesis and presents preliminary results. We introduce the notion of a feasibility problem and explain how it fits into the framework of general optimization problems. In addition to the notions of projectors and reflectors in Section 2.2, we discuss how these constructions can help to solve feasibility problems. In fact, we introduce iterative algorithms including the most common algorithms based on projectors and reflectors in Section 2.3. Among these are the method of alternating projections and the Douglas-Rachford algorithm.

Throughout this thesis, we denote by $\mathcal{E}$ a finite dimensional Euclidean space with inner product $\langle\cdot, \cdot\rangle$ and induced norm $\|\cdot\|$. We denote by $\mathbb{B}$ the open unit ball and by $\mathbb{S}$ the unit sphere in $\mathcal{E}$. The open ball with radius $\delta$ centered around a point $x \in \mathcal{E}$ is denoted by $\mathbb{B}_{\delta}(x) . \mathbb{N}:=\{0,1,2, \ldots\}$ denotes the natural numbers.

\subsection{Feasibility Problems}

The problem of finding a point $x \in \mathcal{E}$ in the intersection of a finite collection of sets is called a feasibility problem. That is, given $m \geq 2$ subsets $\Omega_{1}, \Omega_{2}, \ldots \Omega_{m}$ of $\mathcal{E}$,

$$
\text { find } x \in \bigcap_{i=1}^{m} \Omega_{i} \text {. }
$$

If the sets involved are convex, we call (2.1) a convex feasibility problem. If the intersection of sets $\Omega_{i}$ in $(2.1)$ is nonempty, we say that the feasibility problem is consistent. If, however, the intersection is empty the problem is said to be inconsistent and one is interested in a suitable approximation to each of the involved sets. Depending on what "suitable" means for the problem instance. Feasibility problems are a special case of general optimization problems that consist in minimizing 
a function over some domain. In particular, given a function $f: \mathcal{E} \rightarrow \mathbb{R} \cup\{+\infty\}$ and some set $\Omega \subset \mathcal{E}$, an optimization problem is defined by

$$
\min _{x \in \Omega} f(x) .
$$

That is, we want to find a point $x \in \Omega \subset \mathcal{E}$ that minimizes the function. In particular, by taking $f=\sum_{i=1}^{m} \iota_{i}$ and $\Omega=\mathcal{E}$, where

$$
\iota_{i}(x):= \begin{cases}0 & \text { if } x \in \Omega_{i} \\ +\infty & \text { else }\end{cases}
$$

the minimization problem in (2.2) turns into

$$
\min _{x \in \mathcal{E}} \sum_{i=1}^{m} \iota_{i}(x) .
$$

A point $x \in \mathcal{E}$ is a solution to the feasibility problem (2.1) if and only if it is a solution to the optimization problem (2.3). If the intersection is empty the optimal value of $(2.3)$ is defined as $+\infty$.

\subsection{Projectors And Reflectors}

The distance between two points $x, y \in \mathcal{E}$ associated to $\mathcal{E}$ is given by

$$
\operatorname{dist}(x, y):=\|x-y\|,
$$

whereas

$$
\operatorname{dist}(x, \Omega):=\inf _{y \in \Omega}\|x-y\|,
$$

stands for the distance between the point $x$ and the set $\Omega$. The distance between two subsets $A$ and $B$ of $\mathcal{E}$ is defined as

$$
\operatorname{dist}(A, B):=\inf _{\substack{a \in A \\ b \in B}}\|a-b\| .
$$

The projector onto the set $\Omega$ is given by

$$
P_{\Omega}: \mathcal{E} \rightrightarrows \mathcal{E}: x \mapsto\{y \in \Omega \mid \operatorname{dist}(x, \Omega)=\|y-x\|\},
$$

where $\rightrightarrows$ indicates that $P_{\Omega}$ is a point-to-set mapping. A single element of $P_{\Omega} x$ is called a projection. Note that we restrict our analysis here to representing the distance via the Euclidean norm, i.e. $\operatorname{dist}(x, y)=\|y-x\|$. The existence of projections for all $x \in \mathcal{E}$, i.e. $P_{\Omega} x \neq \emptyset$, is equivalent to the closedness of $\Omega$ (see for instance [51, Theorem 3.1, Theorem 3.8]). Note that the equivalence is due to the finite dimensionality of $\mathcal{E}$. In infinite dimensions closed subspaces do not have to satisfy $P_{\Omega} x \neq \emptyset$ for all points in the space (for an example in a Banach space 


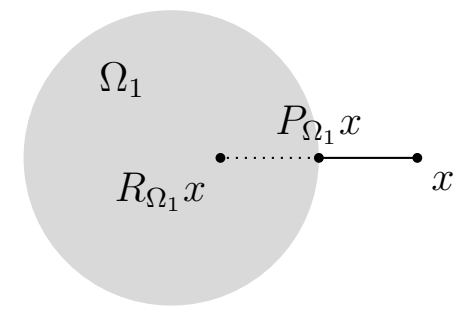

(a) A convex set with its single-valued projector and reflector at $x$.

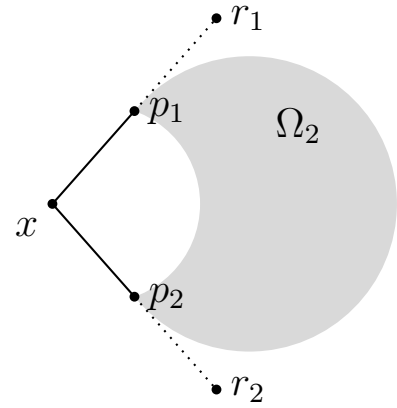

(b) A nonconvex set with a multi-valued projector and reflector at $x$ $\left(P_{\Omega_{2}} x=\left\{p_{1}, p_{2}\right\}\right.$ and $\left.R_{\Omega_{2}} x=\left\{r_{1}, r_{2}\right\}\right)$.

Figure 2.1: Examples of projectors and reflectors.

see [51, Example 3.2]). A set $\Omega$ with the property " $P_{\Omega} x \neq \emptyset$ for all $x \in \mathcal{E}$ " is called a proximal set. Similarly to the projector, we define the reflector across a set $\Omega$ by

$$
R_{\Omega}: \mathcal{E} \rightrightarrows \mathcal{E}: x \mapsto 2 P_{\Omega} x-x
$$

which is again a set-valued mapping. The difference $2 P_{\Omega} x-x$ is taken by the Minkowski difference for the two sets $2 P_{\Omega} x$ and $\{x\}$. That is, given two sets $A$ and $B$ the Minkowski difference is defined by

$$
A-B:=\{a-b \mid a \in A, b \in B\} .
$$

Furthermore, we define the Minkowski sum for $A$ and $B$ by

$$
A+B:=\{a+b \mid a \in A, b \in B\} .
$$

A single element in $R_{\Omega}$ is called a reflection. For an illustration, we consider two sets in $\mathbb{R}^{2}$ as in Fig. 2.1. In the two examples the reflector describes the point $x$ reflected, or mirrored, at the projection onto the set $\Omega_{1}$ and $\Omega_{2}$ respectively.

The regularity of a set has an influence on the properties of the corresponding projector onto the set. Convex sets yield the best properties. A convex set $C$ is defined as a set that contains the line segment $\{\lambda x+(1-\lambda) y \mid \lambda \in(0,1)\}$ for any two points $x, y \in C$. The projector onto convex sets is not only single-valued (see for instance [15, Theorem 3.14]), but satisfies also the following conditions.

Proposition 2.2.1 (characterization of projector onto convex sets and affine subspaces [15, Theorem 3.14, Corollary 3.20]). Let $C \subset \mathcal{E}$ be closed, convex and nonempty. Then the following are equivalent:

(i) $p=P_{C} x$

(ii) $p \in C$ and $\langle y-p, x-p\rangle \leq 0$ for all $y \in C$. 
Moreover, if the set $C$ is an affine subspace, then (ii) holds with equality.

Following the same idea, an analogous result is true for reflectors onto convex sets.

Corollary 2.2.2 (characterization of reflector across convex sets). Let $C \subset \mathcal{E}$ be closed, convex and nonempty. Then the following are equivalent:

(i) $r=R_{C} x$

(ii) $\frac{1}{2}(r+x) \in C$ and $\langle y-r, x-r\rangle \leq \frac{1}{2}\|x-r\|^{2}$ for all $y \in C$.

Proof. This is a direct consequence of Proposition 2.2.1. Let $r=R_{C} x$. By the definition of the reflector given in (2.5), (i) is equivalent to $r=2 P_{C} x-x$. Thus,

$$
P_{C} x=\frac{1}{2}(r+x) \text {. }
$$

Applying Proposition 2.2.1, we deduce that (2.6) is equivalent to $\frac{1}{2}(r+x) \in C$ and

$$
\left\langle y-\frac{1}{2}(r+x), x-\frac{1}{2}(r+x)\right\rangle \leq 0 \quad \text { for all } y \in C .
$$

The last term is nothing more than $\langle y-r, x-r\rangle \leq \frac{1}{2}\|x-r\|^{2}$ for all $y \in C$, by which the result follows.

Given a projector onto a set $\Omega$, the projector onto the shifted set $\Omega+y$, for some $y \in \mathcal{E}$, can be easily determined.

Proposition 2.2.3 (translation formula for projector). Let $\Omega$ be a closed set and $x, y \in \mathcal{E}$. Then

$$
P_{y+\Omega} x=y+P_{\Omega}(x-y) .
$$

Proof. By the definition of the projector given in (2.4), it holds with a few basic reformulations that

$$
\begin{aligned}
P_{y+\Omega} x & =\{z \in \Omega+y \mid \operatorname{dist}(x, \Omega+y)=\|z-x\|\} \\
& =\{z \in \Omega+y \mid \operatorname{dist}(x-y, \Omega)=\|(z-y)-(x-y)\|\} \\
& =\{z+y \in \Omega+y \mid \operatorname{dist}(x-y, \Omega)=\|(z)-(x-y)\|\} \\
& =y+\{z \in \Omega \mid \operatorname{dist}(x-y, \Omega)=\|(z)-(x-y)\|\} \\
& =y+P_{\Omega}(x-y),
\end{aligned}
$$

which proves the claim.

If a set is affine, we get the following fact in addition to the translation formula. 
Proposition 2.2.4 (projector is affine for affine sets). Let $\Omega$ be a closed affine subspace. Then the projector $P_{\Omega}$ is an affine mapping. If, in addition $\Omega$ is linear, then $P_{\Omega}$ is a linear mapping.

Proof. The first part can be found in [15, Corollary 3.20]. For the second part, let $\Omega$ be a closed and linear subspace. Let $x, y \in \mathcal{E}, \alpha, \beta \in \mathbb{R}$, and set $z=\alpha x+\beta y$ and $p=\alpha P_{\Omega} x+\beta P_{\Omega} y$. Since $\Omega$ is linear, we deduce $p \in \Omega$. Now, let $u$ and $v$ be in $\Omega$. By applying Proposition 2.2.1, we derive

$$
\begin{aligned}
\langle u-v, z-p\rangle & =\langle u-v, \alpha x+\beta y-p\rangle \\
& =\alpha\left\langle u-v, x-P_{\Omega} x\right\rangle+\beta\left\langle u-v, y-P_{\Omega} y\right\rangle=0 .
\end{aligned}
$$

Altogether, it follows from Proposition 2.2.1 that $p=P_{\Omega} z$ since a linear subspace is affine as well.

Another concept closely related to that of a projector is the concept of normal cones. For the purpose of this thesis, we define the normal cones in terms of the projector.

Definition 2.2.5 (Normal cones). Let $\Omega \subseteq \mathcal{E}$. Define the smallest cone containing $\Omega$ by

$$
\operatorname{cone}(\Omega):=\mathbb{R}_{+} \cdot \Omega:=\left\{\kappa s \mid \kappa \in \mathbb{R}_{+}, s \in \Omega\right\} .
$$

Let $\Omega \subseteq \mathcal{E}$ and $x \in \Omega$.

(i) The proximal normal cone of $\Omega$ at $x$ is defined by

$$
N_{\Omega}^{\mathrm{prox}}(x)=\operatorname{cone}\left(P_{\Omega}^{-1}(x)-x\right) .
$$

Equivalently, $x^{*} \in N_{\Omega}^{\text {prox }}(x)$ whenever there exists $\sigma \geq 0$ such that

$$
\left\langle x^{*}, y-x\right\rangle \leq \sigma\|y-x\|^{2} \quad(\forall y \in \Omega) .
$$

(ii) The limiting (proximal) normal cone of $\Omega$ at $x$ is defined by

$$
N_{\Omega}(x)=\operatorname{Limsup}_{y \rightarrow x} N_{\Omega}^{\text {prox }}(y),
$$

where the limit superior is taken in the sense of the Painlevé-Kuratowski outer limit (for more details on the outer limit see for instance [118, Chapter 4]).

When $x \notin \Omega$ all normal cones at $x$ are empty (by definition). If the set $\Omega$ is convex, the given normal cones coincide (see for instance [107]). 


\subsection{Projection Methods}

Feasibility problems, as introduced in Section 2.1, are often solved by projectionbased algorithms. In other words, projection-based algorithms are iterative algorithms composed of projectors and reflectors. An iterative algorithm is a procedure generating a sequence $\left(x^{k}\right)_{k \in \mathbb{N}}$, given an initial point $x^{0}$, via a set-valued mapping $T: \mathcal{E} \rightrightarrows \mathcal{E}$ by

$$
x^{k+1} \in T x^{k} .
$$

Remark 2.3.1 (importance of projection based algorithms). Often there are plenty of ways to formulate a problem and, then, how to solve it. The question that arises is, why one should choose to work with projection based algorithms. Recently this was also part of a scientific discussion [43,70,71]. Although there are examples where projection methods are outperformed by specialized solvers [70,71], their outcome in instances where they perform have some advantages as stated by Bauschke and Koch in [21]. In particular, projection methods are easy to understand, easy and inexpensive to implement, easy to maintain, easy to deploy, and can be very fast. Therefore, they are interesting in industrial applications. There are many areas where projection methods can be used such as, road design [21], phase retrieval $[16,17,106]$ (see also chapter Chapter 6), protein reconstruction $[3,60]$, quantum channel construction [58], matrix completion problems [3], and many more. For other examples see $[4,43]$ and references therein.

One of the oldest and simplest algorithms in the form of (2.7) composed of projectors is the method of alternating projections (MAP). The scheme projects back and forth between two closed subsets of $\mathcal{E}$. Its origin dates back to von Neumann who studied the MAP for closed subspaces in [124]. The MAP is defined as follows.

Algorithm 2.3.2 (method of alternating projections). Given an initial point $x^{0}$ and two closed subsets $A$ and $B$ of $\mathcal{E}$, the method of alternating projections generates a sequence $\left(x^{k}\right)_{k \in \mathbb{N}}$ by

$$
x^{k+1} \in T_{\mathrm{MAP}} x^{k}:=P_{B} P_{A} x^{k} \quad(\forall k \in \mathbb{N}) .
$$

There are several works that study the algorithm on convex sets. Cheney and Goldstein were the first analyzing the method of alternating projections without requiring the intersection to be nonempty [45]. Bauschke and Borwein proved linear convergence for bounded linearly regular sets in [11]. This was motivated by the fact that the rate of linear convergence for subspaces is highly dependent on the Friedrichs angle between both sets studied first by Aronszajn in [2] (for the explicit definition of the Friedrichs angle see Definition 4.7.5). A detailed survey can be found in a book of Deutsch [51] and references therein. The first studies for nonconvex sets and the MAP were made by Lewis and Malick who studied smooth 
manifolds in Euclidean spaces in [93] and Lewis et al. in [92] for super-regular sets. This was followed by studies extending the theory on nonconvexity and the regularity of intersections $[22-24,74]$. A natural extension of the MAP to more than two sets is the method of cyclic projections ( $C P$ ) first discovered by Kaczmarz for solving linear systems [83].

Algorithm 2.3.3 (method of cyclic projections). Given an initial point $x^{0}$ and $m \geq 2$ subsets $\Omega_{1}, \Omega_{2}, \ldots, \Omega_{m}$ of $\mathcal{E}$, the method of cyclic projections generates a sequence $\left(x^{k}\right)_{k \in \mathbb{N}}$ by

$$
x^{k+1} \in T_{\mathrm{CP}} x^{k}:=P_{\Omega_{m}} P_{\Omega_{m-1}} \cdots P_{\Omega_{1}} x^{k} \quad(\forall k \in \mathbb{N}) .
$$

The work of Neumann on MAP on two subspaces was extended by Halperin [73] for $\mathrm{CP}$ on finitely many subspaces. The result by Bauschke and Borwein in [11] for linear convergence for bounded linearly regular sets in the 2-set case was generalized by Deutsch and Hundal to $m \geq 2$ sets. They introduced an angle between convex sets [52-54]. Other works including $\mathrm{CP}$ are [13,25,105].

A more sophisticated algorithm is the Douglas-Rachford method (DR). It was first discovered by Douglas and Rachford in a paper about solving a nonlinear heat conduction problem [56]. In the context of feasibility problems the algorithm is defined as follows.

Algorithm 2.3.4 (Douglas-Rachford algorithm). Given an initial point $x^{0}$ and two closed subsets $A$ and $B$ of $\mathcal{E}$, the Douglas-Rachford algorithm generates a sequence $\left(x^{k}\right)_{k \in \mathbb{N}}$ by

$$
x^{k+1} \in T_{A, B} x^{k}:=\left(\frac{1}{2}\left(R_{A} R_{B}+\mathrm{Id}\right)\right) x^{k} \quad(\forall k \in \mathbb{N}) .
$$

Weak convergence of DR was shown by Lion and Mercier [96] in the context of operator splitting for two maximally monotone operators, and was later improved by Svaiter [121]. Linear convergence of DR for affine subspaces was deduced by [76] and thereafter addressed in [19].

Motivated by the performance of DR, Luke proposed and analyzed a convex relaxation of DR in the context of diffraction imaging [97].

Algorithm 2.3.5 (relaxed Douglas-Rachford algorithm). Given an initial point $x^{0}, \lambda \in(0,1)$ and two closed subsets $A$ and $B$ of $\mathcal{E}$, the relaxed Douglas-Rachford algorithm generates a sequence $\left(x^{k}\right)_{k \in \mathbb{N}}$ by

$$
x^{k+1} \in T_{A, B}^{\lambda} x^{k}:=\bigcup_{b \in P_{B} x^{k}}\left\{\frac{\lambda}{2}\left(R_{A}\left(2 b-x^{k}\right)+x^{k}\right)+(1-\lambda) b\right\} \quad(\forall k \in \mathbb{N}) .
$$

We analyze this algorithm in detail in Chapter 4 . The different behavior of the four introduced algorithms for subspaces is illustrated in Fig. 2.2. 


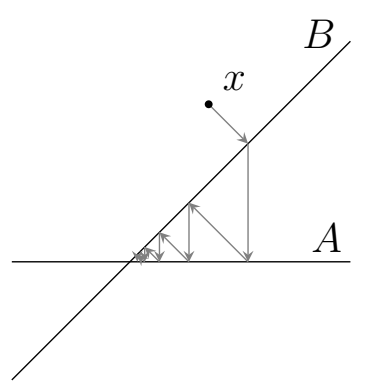

(a) MAP

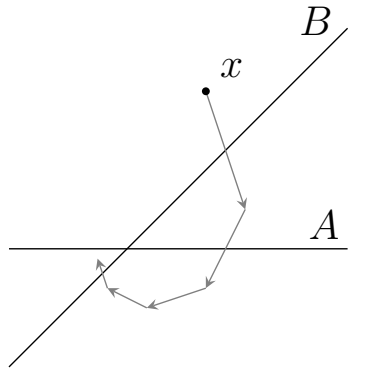

(b) DR

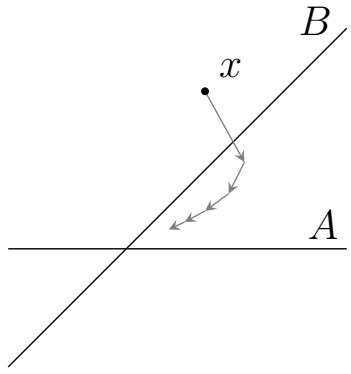

(c) relaxed DR

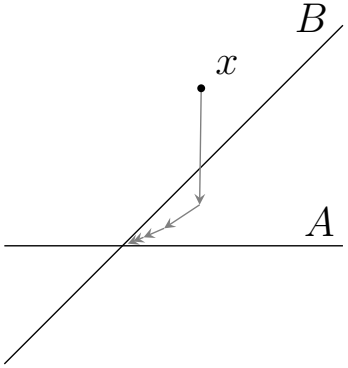

(d) cyc. relaxed DR

Figure 2.2: Illustration of MAP, DR, relaxed DR and its cyclic version $(\lambda=0.4)$ applied to two affine sets $A=\mathbb{R} \times\{0\}$ and $B=\left\{x \in \mathbb{R}^{2} \mid x_{1}=x_{2}\right\}$ with initial point $x=(1,2) \in \mathbb{R}^{2}$ showing 5 iterations.

Whereas it is simple to adapt the method of alternating projections from a 2-set version to its many-set version, the method of cyclic projections, it is not obvious how to do this for algorithms like DR or its relaxed version. Instead, one must use a so-called product space trick that goes back to Pierra [114] that lifts the problem in the product space $\mathcal{E}^{m}$, where $m$ is the number of sets involved. In particular, by defining the two sets

$$
\begin{aligned}
\Omega & :=\left\{x \in \mathcal{E}^{m} \mid x_{i} \in \Omega_{i} \forall i \in\{1,2, \ldots, m\}\right\}, \\
D & :=\left\{x \in \mathcal{E}^{m} \mid x_{1}=x_{2}=\cdots=x_{m}\right\},
\end{aligned}
$$

we can consider the following feasibility problem in $\mathcal{E}^{m}$

$$
\text { find } y \in \Omega \cap D \text {. }
$$

Thus, we reduced an $m$-set problem to a 2 -set problem. Therefore, an advantage of this approach is that we can apply all known algorithms that are restricted to two sets. Moreover, the projectors onto the sets $\Omega$ and $D$ are not more complicated than those onto the sets $\Omega_{1}, \Omega_{2} \ldots, \Omega_{m}$. In fact, the projectors are given by

$$
\begin{aligned}
& P_{\Omega}\left(y_{1}, y_{2}, \ldots, y_{m}\right)=\left\{\left(x_{1}, x_{2}, \ldots, x_{m}\right) \mid x_{i} \in P_{\Omega_{i}} y_{i} \forall i=1,2, \ldots, m\right\} \\
& P_{D}\left(y_{1}, y_{2}, \ldots, y_{m}\right)=\frac{1}{m}\left(\sum_{i=1}^{m} y_{i}, \sum_{i=1}^{m} y_{i}, \ldots, \sum_{i=1}^{m} y_{i}\right) .
\end{aligned}
$$

Note that, when actually computing the projectors $P_{\Omega}$ and $P_{D}$, we only have to compute the projectors $P_{\Omega_{i}}$ for all $i=1,2, \ldots, m$. Thus, we do not need any new knowledge on the problem. However, the increased dimension of the problem, which depends on the number of involved sets $m$ of the original problem in (2.1), does have an impact on the convergence speed. Instead of an $m$-set feasibility problem in $\mathcal{E}$, the problem given in $(2.8)$ is a 2 -set feasibility problem in $\mathcal{E}^{m}$. Furthermore, applying an algorithm in $\mathcal{E}^{m}$ on two sets as in (2.8) yields a fixed point that need not be directly connected to the solution of the same algorithm applied in $\mathcal{E}$. As an example, consider the method of alternating projections. When applied to the 
sets $C$ and $D$ in (2.3.2), the sequence we deduce is nothing more but averaged projections in each entry,

$$
P_{D} P_{\Omega}\left(y_{1}, y_{2}, \ldots, y_{m}\right)=\left(\frac{1}{m} \sum_{i=1}^{m} P_{\Omega_{i}} y_{i}, \frac{1}{m} \sum_{i=1}^{m} P_{\Omega_{i}} y_{i}, \ldots, \frac{1}{m} \sum_{i=1}^{m} P_{\Omega_{i}} y_{i}\right) .
$$

If the sets $D$ and $\Omega$ have an empty intersection, the fixed points of both algorithms do not relate to each other. A different approach to include more than two sets is motivated by the method of cyclic projections. In particular, having an algorithm defined for just two sets, one can apply it several times by interchanging the sets involved cyclically. This idea leads to the cyclic relaxed Douglas-Rachford method.

Algorithm 2.3.6 (cyclic relaxed Douglas-Rachford). Given some point $x^{0} \in \mathcal{E}$, a parameter $\lambda \in(0,1)$ and a finite collection of convex sets $\left\{\Omega_{0}, \Omega_{1}, \ldots, \Omega_{m}\right\}$. Then the relaxed Douglas-Rachford operator on two convex sets $\Omega_{j}, \Omega_{i}$ for $i, j \in$ $\{1,2, \ldots, m\}$ is defined similar as in Algorithm 2.3.5 by

$$
T_{i, j}^{\lambda}:=T_{\Omega_{i}, \Omega_{j}}^{\lambda}=\frac{\lambda}{2}\left(R_{\Omega_{j}} R_{\Omega_{i}}+\mathrm{Id}\right)+(1-\lambda) P_{\Omega_{i}} .
$$

The cyclic relaxed Douglas-Rachford operator is then given by

$$
T_{[1 \ldots m]}^{\lambda}:=T_{m, 1}^{\lambda} \circ \cdots \circ T_{2,3}^{\lambda} \circ T_{1,2}^{\lambda} .
$$

The cyclic relaxed Douglas-Rachford method generates a sequence $\left(x^{k}\right)_{k \in \mathbb{N}}$ through the inclusion $x^{k+1} \in T_{[1 \ldots m]}^{\lambda}\left(x^{k}\right)$.

Note that this definition is explicitly stated for convex sets. To include more general sets one has to define $T_{i, j}^{\lambda}$ similar to Algorithm 2.3.5 to incorporate set-valuedness of the corresponding projectors. We use here this simpler notation, since we concentrate in Chapter 5 on convex sets.

The idea of cyclically composing a certain operator to apply it to more than two sets is not new. Borwein and Tam introduced and analyzed a cyclic Douglas-Rachford method that, unlike Douglas-Rachford on its own, also converges in inconsistent settings in [32]. In the same manner as we did, they interchanged one set in each coupling. Bauschke et al. introduced a variant of this cyclic Douglas-Rachford method that was anchored to one set [25]. That is, instead of changing the role of both sets, one chosen set, the anchor, will always stay the same.

Remark 2.3.7. In addition to its formulation as a feasibility problem in terms of indicator functions, one can always study the more general minimization problem for general functions as mentioned in Section 2.1. The reformulation as an optimization problem in (2.2) where we minimize a function over some domain $\Omega$ is commonly addressed by forward-backward procedures. That is, given some initial point $x^{0}$ define the sequence $\left(x^{k}\right)_{k \in \mathbb{N}}$ via

$$
x^{k+1}=P_{\Omega}\left(x^{k}-\lambda \nabla f\left(x^{k}\right)\right),
$$


where $\lambda$ is a nonnegative constant. Similar to the idea of the simple forwardbackward algorithm, the proximal heterogeneous block implicit-explicit (PHeBIE) method [77], or the proximal alternating linearized minimization (PALM) algorithm [30] are used to solve a constraint optimization problem as in (2.2). Instead of projectors these algorithms may included more general objects like proximal mappings defined by

$$
\operatorname{prox}_{f, \gamma}(x):=\operatorname{argmin}_{y \in \mathcal{E}}\left\{f(y)+\frac{1}{2 \gamma}\|x-y\|\right\}
$$

for a proper lower-semicontinuous convex function $f: \mathcal{E} \rightarrow(-\infty,+\infty]$ and $\gamma>0$. If $f$ is the indicator function $\iota_{\Omega}$ of a closed convex set $\Omega,(2.13)$ simplifies to $P_{\Omega}$ (see for instance [15, Example 12.25]). 


\section{CHAPTER 3}

\section{A Toolkit for Convergence}

In the context of an underlying feasibility or general optimization problem, one usually applies an iterative procedure with the hope to obtain the solution in the limit. Therefore, questions that arise are: Does the algorithm (always) converge? And if so, does it always converge to the same limit point? Is a limit point a solution, or adequate approximation to a solution of the initial problem? Can we quantify the convergence? Can we do this with a certain rate? This chapter aims to address questions like these. Focusing on iterative algorithms defined by a mapping $T$, as in (2.7), we introduce properties of mappings that yield convergence. These are, for example, nonexpansiveness and averagedness, as well as Fejér monotonicity (see Section 3.1). Using these properties, we recall a convergence statement which can be traced back to Opial and, by introducing the notion of metric (sub)regularity, present a related convergence template that is able to quantify convergence (see Section 3.2).

Of special interest in this thesis are feasibility problems and thus projection-based algorithms as presented in Section 2.3. We survey how the regularities of mappings, as discussed in Section 3.1 in the case of projectors, are connected to regularities of sets (see Section 3.3). Beyond that, we examine the implication that regularities of collections of sets have on the rate of convergence. This is established in Section 3.4 by connecting regularity properties of the mapping $T$ to those of the collections of sets involved in the feasibility problem.

\subsection{Regularities of Mappings}

The concept of interest for convergence of iterative algorithms are fixed points. We refer to Fix $T$ as the set of fixed points of the mapping $T$, i.e. $x \in$ Fix $T$ if and only if $x \in T x$. Note that, by this definition, the set $T x$ need not consist entirely of fixed points even when $x \in$ Fix $T$ (see Example 3.1.1). When dealing with fixed points, we want to rule out special cases of those as illustrated in the following example. 
Example 3.1.1 (inhomogenous fixed point set [105, Example 2.1]). Consider the following two sets

$$
\begin{aligned}
& A=\left\{\left(x_{1}, x_{2}\right) \in \mathbb{R}^{2} \mid x_{2} \geq-2 x_{1}+3\right\} \cap\left\{\left(x_{1}, x_{2}\right) \in \mathbb{R}^{2} \mid x_{2} \geq 1\right\} \\
& B=\mathbb{R}^{2} \backslash \mathbb{R}_{++}^{2}
\end{aligned}
$$

as illustrated in Fig. 3.1, and the mapping $T=P_{A} P_{B}$, which defines the method of alternating projections as introduced in Algorithm 2.3.2. In this example

$$
P_{B}(1,1)=\{(0,1),(1,0)\}
$$

and the point $(1,1)$ is a fixed point of $T$ since $(1,1) \in P_{A}\{(0,1)(1,0)\}$. Nevertheless, the point $P_{A}(0,1)$ is also in $T(1,1)$ but not a fixed point of $T$.

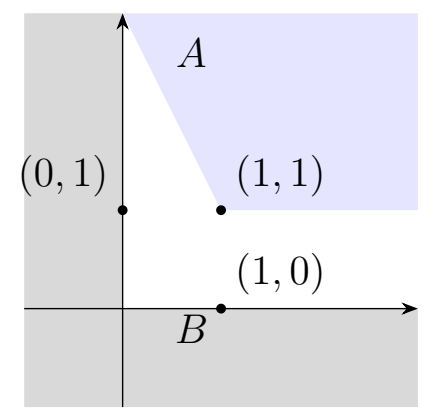

Figure 3.1: Illustration of the sets in Example 3.1.1.

To prevent a case like this, where we stagnate at a point that might not be of interest, we introduce the notions of nonexpansivity and averagedness of a setvalued mapping. The following definition is taken from [105, Definition 2.2].

Definition 3.1.2 (pointwise almost nonexpansive/averaged mappings). Let $D$ be a nonempty subset of $\mathcal{E}$ and let $T: D \rightrightarrows \mathcal{E}$ be a (set-valued) mapping.

(i) $T$ is said to be pointwise almost nonexpansive on $D$ at $y \in D$ if there exists a constant $\epsilon \in[0,1)$ such that

$$
\left\|x^{+}-y^{+}\right\| \leq \sqrt{1+\epsilon}\|x-y\| \quad\left(y^{+} \in T y\right)\left(\forall x^{+} \in T x\right)(\forall x \in D) .
$$

If (3.1) holds with $\epsilon=0$, then $T$ is called pointwise nonexpansive at $y$ on $D$. If $T$ is pointwise (almost) nonexpansive at every point on a neighborhood of $y$ (with the same violation constant $\epsilon$ ) on $D$, then $T$ is said to be (almost) nonexpansive at $y$ (with violation $\epsilon$ ) on $D$.

If $T$ is pointwise (almost) nonexpansive on $D$ at every point $y \in D$ (with the same violation constant $\epsilon$ ), then $T$ is said to be pointwise (almost) nonexpansive on $D$ (with violation $\epsilon$ ). If $D$ is open and $T$ is pointwise (almost) nonexpansive on $D$, then it is (almost) nonexpansive on $D$. 
(ii) $T$ is called pointwise almost averaged on $D$ at $y$ if there is an averaging constant $\alpha \in(0,1)$ and a violation constant $\epsilon \in[0,1)$ such that the mapping $\tilde{T}$ defined by

$$
T=(1-\alpha) \operatorname{Id}+\alpha \tilde{T}
$$

is pointwise almost nonexpansive at $y$ with violation $\epsilon / \alpha$ on $D$.

Similarly, if $\tilde{T}$ is (pointwise) (almost) nonexpansive on $D$ (at $y$ ) (with violation $\epsilon$ ), then $T$ is said to be (pointwise)(almost) averaged on $D$ (at $y$ ) (with averaging constant $\alpha$ and violation $\alpha \epsilon)$.

If the averaging constant $\alpha=\frac{1}{2}$, then $T$ is said to be (pointwise)(almost) firmly nonexpansive on $D$ (with violation $\epsilon$ ) (at $y$ ).

The above definition is very general and does not allow a deep insight in the terminology. However, such a broad description is necessary when working with nonconvex problems. To create a feeling for this condition, we consider the simplest version of the above definitions. In fact, we consider a non-pointwise setting without violation $(\epsilon=0)$.

Definition 3.1.3 (nonexpansive/averaged mappings without violation). Let $D$ be a nonempty subset of $\mathcal{E}$ and let $T: D \rightrightarrows \mathcal{E}$. $T$ is said to be

(i) nonexpansive if

$$
\left\|x^{+}-y^{+}\right\| \leq\|x-y\| \quad(\forall x, y \in D)\left(\forall x^{+} \in T x\right)\left(\forall y^{+} \in T y\right) ;
$$

(ii) averaged if there is a constant $\alpha \in(0,1)$ such that $\tilde{T}$ defined by

$$
T=(1-\alpha) \operatorname{Id}+\alpha \tilde{T}
$$

is nonexpansive. Equivalently, the mapping $T$ is averaged with constant $\alpha$ if the following hold

$$
\begin{aligned}
&\left\|x^{+}-y^{+}\right\| \leq\|x-y\|-\frac{1-\alpha}{\alpha}\left\|\left(x-x^{+}\right)-\left(y-y^{+}\right)\right\| \\
&(\forall x, y \in D)\left(\forall x^{+} \in T x\right)\left(\forall y^{+} \in T y\right),
\end{aligned}
$$

see for instance [15, Proposition 4.25].

Nonexpansivity implies that the mapping $T$ is single-valued (see also [105, Proposition 2.2]). Therefore, we can refer to [15] for a proof of the equivalence in (ii). A version of this property for general set-valued mappings $T$ that are pointwise almost averaged is given in Proposition 3.1.5. The property of being nonexpansive is nothing more than being Lipschitz continuous with constant 1 . That is, given two points, their images under the mapping $T$ are no further away from each other than the initial points. The distance will never increase. In that sense averagedness is a bit stricter. Here, an additional violation has to be satisfied. Thus, every averaged mapping is nonexpansive as well. Both properties have a great impact on the properties of the set of fixed points as well as on the convergence analysis of the related Picard iteration as presented in this and the following section. 
Example 3.1.4. The identity mapping Id might be the most trivial example of a nonexpansive mapping. Moreover, Id is averaged for any constant $\alpha \in(0,1)$. This is clear from Definition 3.1.3.

The following statement allows us to characterize averagedness in various ways.

Proposition 3.1.5 (characterization of almost averaged mappings [105, Proposition 2.1]). Let $T: \mathcal{E} \rightrightarrows \mathcal{E}, U \subset \mathcal{E}$ and let $\alpha \in(0,1)$. The following are equivalent:

(i) $T$ is pointwise almost averaged at $y$ on $U$ with violation $\epsilon$ and averaging constant $\alpha$.

(ii) $\left(1-\frac{1}{\alpha}\right) \operatorname{Id}+\frac{1}{\alpha} T$ is pointwise almost nonexpansive at $y$ on $U \subset \mathcal{E}$ with violation $\epsilon / \alpha$.

(iii) For all $x, y \in U, x^{+} \in T x$ and $y^{+} \in T y$ it holds that

$$
\left\|x^{+}-y^{+}\right\|^{2} \leq(1+\epsilon)\|x-y\|^{2}-\frac{1-\alpha}{\alpha}\left\|\left(x-x^{+}\right)-\left(y-y^{+}\right)\right\|^{2} .
$$

Consequently, if $T$ is pointwise almost averaged at $y$ on $U$ with violation $\epsilon$ and averaging constant $\alpha$, then $T$ is pointwise almost nonexpansive at $y$ on $U$ with violation $\epsilon$.

Using Proposition 3.1.5, we can show that the projection onto a closed convex set is averaged.

Proposition 3.1.6 (projection onto a convex set is averaged). Let $C \subset \mathcal{E}$ be closed and convex. Then its projector satisfies the following inequality.

$$
\left\|P_{C} x-P_{C} y\right\|^{2}+\left\|\left(\operatorname{Id}-P_{C}\right)(x)-\left(\operatorname{Id}-P_{C}\right)(y)\right\|^{2} \leq\|x-y\|^{2} \quad(\forall x, y \in \mathcal{E}) .
$$

Consequently, the projector onto the set $C$ is averaged with constant $\alpha=1 / 2$.

Proof. This is a simple implication of Proposition 2.2.1 and Proposition 3.1.5.

Next we see that having several averaged mappings, their composition is again averaged.

Proposition 3.1.7 (compositions of averages of relatively averaged operators [105, Proposition 2.10]). Let $T_{j}: \mathcal{E} \rightrightarrows \mathcal{E}$ for $j=1,2, \ldots, m$ be pointwise almost averaged on $U_{j}$ at all $y_{j} \in S_{j} \subset \mathcal{E}$ with violation $\epsilon_{j}$ and averaging constant $\alpha_{j} \in(0,1)$, where $U_{j} \supset S_{j}$ for $j=1,2, \ldots, m$.

(i) If $U:=U_{1}=U_{2}=\cdots=U_{m}$ and $S:=S_{1}=S_{2}=\cdots=S_{m}$, then the weighted mapping $T:=\sum_{j=1}^{m} w_{j} T_{j}$ with weights $w_{j} \in[0,1], \sum_{j=1}^{m} w_{j}=1$, is pointwise almost averaged at all $y \in S$ with violation $\epsilon=\sum_{j=1}^{m} w_{j} \epsilon_{j}$ and averaging constant $\alpha=\max _{j=1,2, \ldots, m}\left\{\alpha_{j}\right\}$ on $U$. 
(ii) If $T_{j} U_{j} \subseteq U_{j-1}$ and $T_{j} S_{j} \subseteq S_{j-1}$ for $j=2,3, \ldots, m$, then the composed mapping $T:=T_{1} \circ T_{2} \circ \cdots \circ T_{m}$ is pointwise almost nonexpansive at all $y \in S_{m}$ on $U_{m}$ with violation at most

$$
\epsilon=\prod_{j=1}^{m}\left(1+\epsilon_{j}\right)-1 .
$$

(iii) If $T_{j} U_{j} \subseteq U_{j-1}$ and $T_{j} S_{j} \subseteq S_{j-1}$ for $j=2,3, \ldots, m$, then the composed mapping $T:=T_{1} \circ T_{2} \circ \cdots \circ T_{m}$ is pointwise almost averaged at all $y \in S_{m}$ on $U_{m}$ with violation at most $\epsilon$ given by (3.2) and averaging constant at least

$$
\alpha=\frac{m}{m-1+\frac{1}{\max _{j=1,2, \ldots, m}\left\{\alpha_{j}\right\}}} .
$$

Example 3.1.8 (averaged mappings [105, Example 2.2(iv)]). We consider again the setting in Example 3.1.1. That is, $T:=P_{A} P_{B}$ and the sets

$$
\begin{aligned}
& A=\left\{\left(x_{1}, x_{2}\right) \in \mathbb{R}^{2} \mid x_{2} \geq-2 x_{1}+3\right\} \cap\left\{\left(x_{1}, x_{2}\right) \in \mathbb{R}^{2} \mid x_{2} \geq 1\right\} \\
& B=\mathbb{R}^{2} \backslash \mathbb{R}_{++}^{2} .
\end{aligned}
$$

In this case, $T$ is not pointwise almost averaged at $(1,1)$ for any $\epsilon>0$. In light of Example 3.1.1, this shows that the pointwise almost averaged property is incompatible with fixed points whose image under the mapping $T$ contains other points than fixed points.

Example 3.1.1 and Example 3.1.8 highlight that we have to rule fixed points whose image under the mapping $T$ includes more than just fixed points. Exploiting the property of being averaged we can avert such instances.

Proposition 3.1.9 (single-valuedness of averaged mappings [105, Proposition 2.2]). Let $T: \mathcal{E} \rightrightarrows \mathcal{E}$ be pointwise almost averaged on $D \subset \mathcal{E}$ at $\bar{x} \in D$ with violation $\epsilon \geq 0$. Then $T$ is single-valued at $\bar{x}$. In particular, if $\bar{x} \in$ Fix $T$, then $T \bar{x}=\{\bar{x}\}$.

Related to the concept of averagedness, but different, are Fejér monotonicity and asymptotic regularity.

Definition 3.1.10 (Fejér monotonicity). Let $\Omega$ be a nonempty subset of $\mathcal{E}$ and $\left(x^{k}\right)_{k \in \mathbb{N}}$ be a sequence in $\mathcal{E}$. Then $\left(x^{k}\right)_{k \in \mathbb{N}}$ is called Fejér monotone with respect to $\Omega$ if

$$
\left\|x^{k+1}-y\right\| \leq\left\|x^{k}-y\right\| \quad(\forall y \in \Omega)(\forall k \in \mathbb{N}) .
$$

Definition 3.1.11 (asymptotic regularity). Let $T: \mathcal{E} \rightarrow \mathcal{E}$ and $x^{0} \in \mathcal{E}$. Define the sequence $\left(x^{k}\right)_{k \in \mathbb{N}}$ via $x^{k+1} \in T x^{k}$ for all $k \in \mathbb{N}$. Then $\left(x^{k}\right)_{k \in \mathbb{N}}$ is called asymptotically regular if

$$
x^{k+1}-x^{k} \rightarrow 0 .
$$


Proposition 3.1.12 (averagedness implies Fejér monotonicity and asymptotic regularity). Let $T: \mathcal{E} \rightarrow \mathcal{E}$ be an averaged mapping with Fix $T \neq \emptyset$. Let $x^{0} \in \mathcal{E}$ and define the sequence $\left(x^{k}\right)_{k \in \mathbb{N}}$ by $x^{k+1} \in T x^{k}$. Then $\left(x^{k}\right)_{k \in \mathbb{N}}$ is Fejér monotone with respect to Fix $T$ and $x^{k+1}-x^{k} \rightarrow 0$.

Proof. This is a special case of [15, Proposition 5.15].

\subsection{Convergence Theorems}

So far, we have seen different kinds of algorithms for treating feasibility problems and some regularity properties of the involved mappings. This section aims to explore conditions that provide convergence. It turns out that convergence itself routes back to regularity properties of the mapping. In application, however, one often wants not only convergence but to be able to quantify it. This leads to a second essential property that is called metric (sub-)regularity. The basic convergence idea goes back to Opial [110]. Applying his result to our setting yields the following statement.

Theorem 3.2.1. Let $T: \mathcal{E} \rightrightarrows \mathcal{E}$ be averaged with Fix $T \neq \emptyset$. Then the sequence $\left(x^{k}\right)_{k \in \mathbb{N}}$ defined by $x^{k+1} \in T x^{k}$ converges to a point in Fix $T$ for any $x^{0} \in \mathcal{E}$.

Proof. Using averagedness of $T$ and Proposition 3.1.9, we deduce single-valuedness of the mapping. Moreover, since $T$ is averaged, we can apply Proposition 3.1.12 and deduce that the sequence $\left(x^{k}\right)_{k \in \mathbb{N}}$ is Fejér monotone and asymptotically regular. Applying Proposition 3.1.9, yields single-valuedness of the mapping $T$. The result then follows by Opial's original statement [110, Theorem 1].

Henceforth, we will see that averagedness of $T$ and a nonempty fixed point set is enough to get convergence. As one would expect, it can be difficult for a map to satisfy these properties globally (see Example 3.1.8). Nevertheless, this is often the case in nonconvex problem instances. Thus, we seek a statement that includes local properties. That is in our case pointwise almost averagedness as in Definition 3.1.2.

In addition to mere convergence, it is convenient to know how fast the sequence converges. We are especially interested in linear convergence. The following types of linear convergence can be found in [111, Chapter 9].

Definition 3.2.2 (types of linear convergence). Let $\left(x^{k}\right)_{k \in \mathbb{N}}$ be a sequence in $\mathcal{E}$. We say that the sequence $\left(x^{k}\right)_{k \in \mathbb{N}}$ converges

(i) R-linearly to a point $y$ with rate $c \in[0,1)$ if there exists a constant $\gamma>0$ such that

$$
\left\|x^{k}-y\right\| \leq \gamma c^{k} \quad(\forall k \in \mathbb{N})
$$


(ii) Q-linearly to a point $y$ with rate $c \in[0,1)$ if

$$
\left\|x^{k+1}-y\right\| \leq c\left\|x^{k}-y\right\| \quad(\forall k \in \mathbb{N}) .
$$

In the context of iterative algorithms and the sequences they produce, one needs an additional assumption besides averagedness that also relates to the stability of the fixed points. Here, we focus on metric regularity of set-valued mappings. This concept has been studied by many authors in the literature (see for instance $[5,55$, $78,79,112,118])$. For the definition of metric regularity we need gauge functions. A function $\mu:[0, \infty) \rightarrow[0, \infty)$ is a gauge function if it is continuous and strictly increasing with $\mu(0)=0$ and $\lim _{t \rightarrow \infty} \mu(t)=\infty$.

Definition 3.2.3 (metric regularity on a set [105, Definition 2.5]). Let $\Phi: \mathcal{E} \rightrightarrows \mathcal{Y}$, $U \subset \mathcal{E}, V \subset \mathcal{Y}$, where $\mathcal{Y}$ is a Euclidean space. Furthermore, let $\mu:[0, \infty) \rightarrow[0, \infty)$ be a gauge function. The mapping $\Phi$ is called metrically regular with gauge $\mu$ on $U \times V$ relative to $\Lambda \subset \mathcal{E}$ if

$$
\operatorname{dist}\left(x, \Phi^{-1}(y) \cap \Lambda\right) \leq \mu(\operatorname{dist}(y, \Phi(x)))
$$

holds for all $x \in U \cap \Lambda$ and $y \in V$ with $0<\mu(\operatorname{dist}(y, \Phi(x)))$. When the set $V$ consists of a single point, $V=\{\bar{y}\}$, then $\Phi$ is said to be metrically subregular for $\bar{y}$ on $U$ with gauge $\mu$ relative to $\Lambda \subset \mathcal{E}$.

When $\mu$ is linear on $[0, \infty)$ (that is, $\mu(t)=\kappa t, \forall t \in[0, \infty)$ ), one says "with constant $\kappa$ " instead of "with gauge $\mu(t)=\kappa t$ ". When $\Lambda=\mathcal{E}$, the quantifier "relative to" is dropped. When $\mu$ is linear, the smallest constant $\kappa$ for which (3.4) holds is called modulus of metric regularity.

Metric subregularity provides the existence of a local error bound (see $[80,86]$ ) and is thus connected to this area of research. Moreover, metric regularity of a set-valued mapping is equivalent to the Kurdyka-Łojasiewicz property of a related single-valued function (see for instance [29]). A different way to understand metric subregularity is its equivalent formulation by inverse calmness (see for instance [55, Section 3H.]).

Remark 3.2.4. Note, that in the subsequent analysis, such as Theorem 3.2.6 and the results on relaxed Douglas-Rachford in Chapter 4, we only consider the case when $\mu$ is linear. Since the type of function of $\mu$ has an impact on the convergence (see for instance [105, Theorem 2.2]), we limit the analysis to linear types of convergence.

While Definition 3.2.3 might seem abstract, there are sufficient conditions for metric regularity or reformulations that allow to prove metric regularity. One of these is polyhedrality. A mapping $T: \mathcal{E} \rightrightarrows \mathcal{E}$ is called polyhedral if its graph is the union of finitely many sets that can be expressed as the intersection of finitely many closed half-spaces and/or hyperplanes [55]. 
Proposition 3.2.5 (polyhedrality implies metric subregularity [105, Proposition 2.6]). Let $\Lambda \subset \mathcal{E}$ be an affine subspace and $T: \Lambda \rightrightarrows \Lambda$. If $T$ is polyhedral and Fix $T \cap \Lambda$ is an isolated point, $\{\bar{x}\}$, then $\Phi:=T-\mathrm{Id}$ is metrically subregular for 0 on $U$ (metrically regular on $U \times\{0\}$ ) relative to $\Lambda$ with some constant $\kappa$ for some neighborhood $U$ of $\bar{x}$. In particular, $U \cap \Phi^{-1}(0)=\{\bar{x}\}$.

Collecting the concepts we have established so far, we present the following convergence result that goes back to Luke, Tam and Thao in [105, Theorem 2.2] and was later refined in [104] by Luke, Teboulle and Thao to convergence to a specific point.

Theorem 3.2.6 ((sub)linear convergence with metric subregularity). Let $T: \Lambda \rightrightarrows$ $\Lambda$ for $\Lambda \subset \mathcal{E}$, with Fix $T$ nonempty and closed, $\Phi:=T-\operatorname{Id}$ and set $S_{\delta}:=$ (Fix $T+\delta \mathbb{B}) \cap \Lambda$ for $\delta \in \mathbb{R}_{+}$. Suppose that, for all $\bar{\delta}>0$ small enough, there is a $\gamma \in(0,1), \epsilon>0$ and an $\alpha \in(0,1)$, such that,

(i) $T$ is pointwise almost averaged at all $y \in \mathrm{Fix} T \cap \Lambda$ with averaging constant $\alpha$ and violation $\epsilon$ on $S_{\gamma \bar{\delta}}$, and

(ii) for $\bar{S}:=S_{\gamma \bar{\delta}} \backslash$ Fix $T$, $\Phi$ is metrically subregular for 0 on $\bar{S}$ with constant $\kappa$ relative to $\Lambda$.

Then for any $x^{0} \in \Lambda$ close enough to Fix $T \cap \Lambda$, the iterates $x^{j+1} \in T x^{j}$ satisfy

$$
\operatorname{dist}\left(x^{j+1}, \text { Fix } T \cap \Lambda\right) \leq c \operatorname{dist}\left(x^{j}, \text { Fix } T \cap \Lambda\right) \quad \forall x^{j} \in \bar{S},
$$

where $c:=\sqrt{1+\epsilon-\left(\frac{1-\alpha}{\kappa^{2} \alpha}\right)}$. If, in addition $\kappa$ satisfies

$$
\kappa<\sqrt{\frac{1-\alpha}{\epsilon \alpha}},
$$

then $\operatorname{dist}\left(x^{j}, \tilde{x}\right) \rightarrow 0$ for some $\tilde{x} \in \mathrm{Fix} T \cap \Lambda$ at least $R$-linearly with rate at most $c<1$. If Fix $T \cap \Lambda$ is a single point, then convergence is $Q$-linear.

Remark 3.2.7. In both Theorem 3.2.1 as well as Theorem 3.2.6(i) averagedness is the essential property for convergence of iterative algorithms. Whereas assumption (ii) of Theorem 3.2.6 serves to quantify the convergence, the inequality in (3.5) is closely related to the property of being paracontractive. A continuous mapping $T: \mathbb{R}^{n} \rightarrow \mathbb{R}^{n}$ is paracontractive (see [61]) if for any $x \in \mathbb{R}^{n} \backslash$ Fix $T$ and $x^{+}=T x$ it holds

$$
\operatorname{dist}\left(x^{+}, y\right)<\operatorname{dist}(x, y) \quad(\forall y \in \operatorname{Fix} T) .
$$

Hence, if $T$ is single-valued, which is the case at points where $T$ is averaged, and $\mathcal{E}=\mathbb{R}^{n}, \Lambda=$ Fix $T$, then (3.7) implies (3.5). 


\subsection{Regularities of Sets}

In the previous sections we have seen that regularities of a mapping $T$ imply convergence of the iterative algorithm corresponding to $T$. What is missing, is the connection to feasibility problems and projection based algorithms. The link between these two areas is made by regularity concepts on the sets themselves, but also the whole collection of sets involved. The former concept is treated in this section.

There are numerous definitions available to work with nonconvex sets. A recent survey by Kruger et al. [88] sorted the different classes of nonconvex sets to highlight their dependencies and differences. By uniting several concepts of regularities for sets, we propose to use the notion of $\epsilon$-set regularity as introduced in [88] and refined in [48].

Definition 3.3.1 ( $\epsilon$-subregularity [48, Definition 2.2]). A set $\Omega$ is $\epsilon$-subregular relative to $\Lambda \subset \mathcal{E}$ at $\bar{x} \in \Omega$ for $(x, v) \in \operatorname{gph} N_{\Omega}$ if it is locally closed at $\bar{x}$, and there exists an $\epsilon>0$ together with a neighborhood $U_{\epsilon}$ of $\bar{x}$, such that

$$
\left\langle v-\left(y^{\prime}-y\right), y-x\right\rangle \leq \epsilon\left\|v-\left(y^{\prime}-y\right)\right\|\|y-x\| \quad\left(\forall y^{\prime} \in \Lambda \cap U_{\epsilon}\right)\left(\forall y \in P_{\Omega} y^{\prime}\right),
$$

where gph $T$ denotes the graph of the mapping $T: \mathcal{E} \rightrightarrows \mathcal{E}$ defined by $\operatorname{gph} T:=$ $\{(x, y) \in \mathcal{E} \times \mathcal{E} \mid y \in T x\}$. $\Omega$ is called subregular relative to $\Lambda$ at $\bar{x}$ for $(x, v) \in$ gph $N_{\Omega}$ if it is locally closed and for all $\epsilon>0$ there exists $U_{\epsilon}$ such that (3.8) holds. If $\Lambda=\{\bar{x}\}$, then the qualifier "relative to" is dropped.

Example 3.3.2 ( $\epsilon$-subregularity). (i) Circles, [105, Example 3.1.b]. Consider the set

$$
A=\left\{\left(x_{1}, x_{2}\right) \in \mathbb{R}^{2} \mid x_{1}^{2}+x_{2}^{2}=1\right\} .
$$

The set $A$ is subregular at any $\bar{x} \in A$ for all $(\bar{x}, v) \in \operatorname{gph} N_{A}$. To see this, note first that for any $\bar{x} \in A, N_{A}(\bar{x})$ consists of a line passing through the origin and the point $\widehat{x}$. Now, for any $\epsilon \in(0,1)$, we choose $\delta=\epsilon$. Then, for any $x \in A \cap \mathbb{B}_{\delta}(\bar{x})$, it holds $\cos \angle(-\bar{x}, x-\bar{x}) \leq \delta \leq \epsilon$. Hence, for all $x \in A \cap \mathbb{B}_{\delta}(\bar{x})$ and $v \in N_{A}(\bar{x})$,

$$
\begin{aligned}
\langle v, x-\bar{x}\rangle & =\cos \angle(v, x-\bar{x})\|v\|\|x-\bar{x}\| \\
& \leq \cos \angle(-\bar{x}, x-\bar{x})\|v\|\|x-\bar{x}\| \\
& \leq \epsilon\|v\|\|x-\bar{x}\| .
\end{aligned}
$$

(ii) Convex sets. Let $A$ be a closed and convex set. By Proposition 2.2.1 it holds

$$
\langle v, x-\bar{x}\rangle \leq 0 \quad(\forall x \in A)\left(\forall v \in N_{A}(\bar{x})\right) .
$$

Thus, $A$ is subregular with neighborhood $U=\mathcal{E}$. 
Of special interest in this thesis are super-regular sets and their extension superregular sets at a distance. The latter notion is a version of the former relative to points not belonging to the set.

Definition 3.3.3 (super-regularity [92, Definition 4.3]). Let $\Omega \subseteq \mathbb{R}^{n}$ and $\bar{x} \in \Omega$. The set $\Omega$ is said to be super-regular at $\bar{x}$ if it is locally closed at $\bar{x}$, and for every $\epsilon>0$ there is a $\delta>0$ such that for all $(x, 0) \in \operatorname{gph} N_{\Omega} \cap\left\{\left(\mathbb{B}_{\delta}(\bar{x}), 0\right)\right\}$

$$
\left\langle y^{\prime}-y, x-y\right\rangle \leq \varepsilon\left\|y^{\prime}-y\right\|\|x-y\| \quad\left(\forall y^{\prime} \in \mathbb{B}_{\delta}(\bar{x})\right)\left(\forall y \in P_{\Omega} y^{\prime}\right) .
$$

Remark 3.3.4 ( $\epsilon$-subregularity, super-regularity and other notions). By (3.9) in Definition 3.3.1, it is easy to see that every set $\Omega$ that is super-regular at $\bar{x} \in \Omega$, is also $\epsilon$-subregular at $\bar{x}$ for all $(x, 0) \in \operatorname{gph} N_{\Omega} \cap\left\{\left(\mathbb{B}_{\delta}(\bar{x}), 0\right)\right\}$. Thus, the notion of $\epsilon$ subregularity captures super-regular sets. Moreover, there are other notions that fit into the framework of $\epsilon$-subregularity. This was shown in [88] where $\epsilon$-subregularity was called elemental (sub-)regularity. The authors proved that Clarke regularity, prox-regularity, convexity and others imply elemental (sub-)regularity. They also showed that these regularity notions do imply each other in the following order: convexity $\Rightarrow$ prox-regularity $\Rightarrow$ super-regularity $\Rightarrow$ Clarke regularity (see $[88]$ and references therein).

Rewriting Definition 3.3.3 leads to the following equivalent characterization of super-regularity, which is more useful for our purposes.

Proposition 3.3.5 ([92, Proposition 4.4]). The set $\Omega \subseteq \mathcal{E}$ is super-regular at $\bar{x} \in \Omega$ if and only if it is locally closed at $\bar{x}$, and for every $\varepsilon>0$ there exists $\delta>0$ such that

$$
\begin{array}{r}
\langle v, x-y\rangle \leq \varepsilon\|v\|\|x-y\| \\
\left.\qquad \forall(x, v) \in \operatorname{gph} N_{\Omega} \cap\left(\mathbb{B}_{\delta}(\bar{x}) \times \mathcal{E}\right)\right)\left(\forall y \in \Omega \cap \mathbb{B}_{\delta}(\bar{x})\right) .
\end{array}
$$

To extend super-regularity to super-regularity at a distance, we employ the notion of $\epsilon$-subregular sets.

Definition 3.3.6 (super-regularity at a distance). A set $\Omega$ is called $\epsilon$-super-regular at a distance relative to $\Lambda \subset \mathcal{E}$ at $\bar{x}$ if it is $\epsilon$-subregular relative to $\Lambda$ at $\bar{x}$ for all $(x, v) \in V_{\epsilon}$, where

$$
V_{\epsilon}:=\left\{(x, v) \in \operatorname{gph} N_{\Omega}^{\text {prox }} \mid x+v \in U_{\epsilon}, x \in P_{\Omega}(x+v)\right\},
$$

and $U_{\epsilon}$ is a neighborhood of $\bar{x}$. $\Omega$ is called super-regular at a distance relative to $\Lambda$ at $\bar{x}$ if it is $\epsilon$-super-regular at a distance relative to $\Lambda$ at $\bar{x}$ for all $\epsilon>0$.

Comparing Proposition 3.3.5 and Definition 3.3.6 super-regularity is more strict than super-regularity at a distance. Note that implicitly $U_{\epsilon} \cap \Lambda \neq \emptyset$ for all $\epsilon>$ 0 . The notion of super-regularity at a distance is of particular importance for our analysis of the relaxed Douglas-Rachford algorithm. In fact, we will see in Chapter 4 that the fixed points of the relaxed Douglas-Rachford mapping $T_{A, B}^{\lambda}$ need not be contained in $A$ and $B$. Thus, we have to describe the regularity of the sets relative to points not (necessarily) in the sets. 


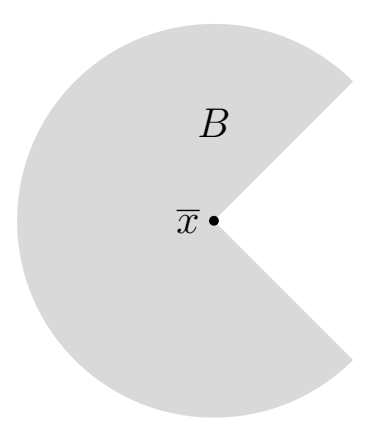

Figure 3.2: Illustration of the sets in Example 3.3.8.

Remark 3.3.7 (super-regularity at a distance relative to $\mathcal{E}$ is equivalent to super-regularity). Super-regularity at a distance relative to $\Lambda=\mathcal{E}$ at some point $\bar{x} \in \Omega$ implies super-regularity at $\bar{x}$. To see this, let $\Omega$ be super-regular at a distance relative to $\Lambda=\mathcal{E}$ at $\bar{x} \in \Omega$. For fixed $\epsilon>0$ note that $(x, 0) \in V_{\epsilon}$ for all $x \in \Omega \cap U_{\epsilon}$. With these, (3.8) becomes

$$
\left\langle y-y^{\prime}, y-x\right\rangle \leq \epsilon\left\|y-y^{\prime}\right\|\|y-x\|
$$

for all $y^{\prime} \in \Lambda \cap U_{\epsilon}, y \in P_{\Omega} y$ and for all $x \in U_{\epsilon} \cap \Omega$. Certainly, there exists an $\delta>0$ such that $\mathbb{B}_{\delta} \subset U_{\epsilon}$. Moreover, since $\Lambda=\mathcal{E}$ (3.12) holds for all $y^{\prime} \in U_{\epsilon}, y \in P_{\Omega} y$ and for all $x \in U_{\epsilon} \cap \Omega$, which is by Definition 3.3.3 super-regularity of $\Omega$ at $\bar{x}$. The other implication follows just by the definition of both regularities.

Note that taking $\Lambda=\mathcal{E}$ was essential to prove the equivalence. If we restrict $\Lambda$ to a proper subset of $\mathcal{E}$, the equivalence does not have to hold anymore, as we see in the next example.

Example 3.3.8 (super-regularity at a distance does not imply super-regularity). This example considers Pac-Man.

$$
\begin{aligned}
& B=\left\{\left(x_{1}, x_{2}\right) \in \mathbb{R}^{2}\left|x_{1}^{2}+x_{2}^{2} \leq 1, x_{1} \leq\right| x_{2} \mid\right\} \subset \mathbb{R}^{2} \\
& \bar{x}=(0,0) .
\end{aligned}
$$

The set $B$ is super regular at a distance relative to $P_{B}^{-1}(\bar{x})$ at $\bar{x}$. But it is not superregular at $\bar{x}$. To show the first assertion, we take in Definition 3.3.6 $\Lambda=P_{B}^{-1}(\bar{x})$, $\epsilon=0$ and the neighborhood $U_{\epsilon}=\mathcal{E}$. Note that $a^{\prime} \in P_{B}^{-1}(\bar{x})$ implies $a^{\prime}=\bar{x}$. Then, for all $(b, v) \in V_{\epsilon}$ and for all $a^{\prime} \in A, a \in P_{B} a^{\prime}$ we note that

$\left\langle v-\left(a^{\prime}-a\right), a-b\right\rangle=\langle v, a-b\rangle-\left\langle a^{\prime}-a, a-b\right\rangle=\langle v, \bar{x}-b\rangle-\langle 0, \bar{x}-b\rangle=0=\epsilon$.

which shows that $B$ is super regular at a distance relative to $P_{B}^{-1}(\bar{x})$ at $\bar{x}$. To prove that $B$ is not super-regular at $\bar{x}$, we argue similar to [48, Example 2.7]. Take any $(0, v) \in N_{B}$ and $y=t v$ with $0 \neq v \in B \cap N_{B}(0)$ and $t \downarrow 0$. Then

$$
\langle v, y\rangle=t\langle v, v\rangle=t\|v\|>0 .
$$

By Proposition 3.3.5, we conclude that $B$ is not super-regular at $\bar{x}$. 
Besides this example the strength of the notion of super-regularity at a distance becomes more obvious in Proposition 3.3.12 where we analyze the regularity of projectors and reflectors on super-regular sets at a distance relative to points not in the set.

Proposition 3.3.9 (convex sets are super-regular at a distance). Let $\Omega \subset \mathcal{E}$ be convex and closed. Then $\Omega$ is super-regular at a distance relative to $\Lambda=\mathcal{E}$ at any $\bar{x} \in \Omega$.

Proof. Fix $\bar{x} \in \Omega$. For convex sets one has

$$
\langle v, x-y\rangle \leq 0 \quad(\forall x, y \in \Omega)\left(\forall v \in N_{\Omega}(y)\right) .
$$

Thus, for any open set $U \subset \mathcal{E}, y^{\prime} \in U$, and $y \in P_{\Omega} y^{\prime}$, which implies that $y^{\prime}-y \in$ $N_{\Omega}(y)$. From this we deduce that $\left\langle y^{\prime}-y, x-y\right\rangle \leq 0$ and thus

$$
\left\langle v-\left(y^{\prime}-y\right), y-x\right\rangle \leq 0 \quad\left(\forall y^{\prime} \in \Lambda \cap U_{\epsilon}\right)\left(\forall y \in P_{\Omega} y^{\prime}\right)
$$

This shows super-regularity at a distance of $\Omega$ relative to $\mathcal{E}$ at all $\bar{x} \in \Omega$ as claimed.

Example 3.3.10 (circles are super-regular at a distance). Consider the set

$$
\Omega:=\left\{\left(x_{1}, x_{2}\right) \in \mathbb{R}^{2} \mid x_{1}^{2}+x_{2}^{2}=1\right\} .
$$

This set is $\epsilon$-subregular relative to $\Lambda=P_{\Omega}^{-1}(\bar{x})$ at any $\bar{x} \in \Omega$ for all $(\bar{x}, v) \in \operatorname{gph} N_{\Omega}$ with $\epsilon=0$ (which implies that $\Omega$ is in fact subregular relative to $\Lambda$ for all $(\bar{x}, v) \in$ gph $\left.N_{\Omega}\right)$ as seen in Example 3.3.2(i). Indeed, for any $\delta \in(0,1)$ we have, for any $y^{\prime} \in \Lambda \cap \mathbb{B}_{\delta}(\bar{x})$, that $y \in P_{\Omega} y^{\prime}$ is given by $y=\bar{x}$ and (3.8) specializes to

$$
\left\langle v-\left(y^{\prime}-y\right), y-\bar{x}\right\rangle=\left\langle v-\left(y^{\prime}-y\right), \bar{x}-\bar{x}\right\rangle=0 \quad\left(\forall y^{\prime} \in \Lambda \cap \mathbb{B}_{\delta}(\bar{x})\left(\forall v \in N_{\Omega}(\bar{x})\right) .\right.
$$

Moreover, the set $\Omega$ is super-regular at a distance relative to $\Lambda=P_{\Omega}^{-1}(\bar{x})$ at any $\bar{x} \in \Omega$. To see this, we first show that $\Omega$ is $\epsilon$-super-regular at a distance relative to $P_{\Omega}^{-1}(\bar{x})$ at $\bar{x}$ for any $\epsilon \in(0,0.5)$. Fix a $\epsilon \in(0,0.5)$ and set $\delta=2 \epsilon$. For any $w \in N_{\Omega}(\bar{x})$ and $x \in \Omega \cap \mathbb{B}_{\delta}(\bar{x})$ it holds $\cos \angle(w, x-\bar{x}) \leq \cos \angle(-\bar{x}, x-\bar{x})$. By the law of cosine we conclude $\cos \angle(-\bar{x}, \bar{x}-x)=\|\bar{x}-x\| / 2<\delta / 2<\epsilon$. Since $v-\left(y^{\prime}-\bar{x}\right) \in N_{\Omega}(\bar{x})$ for all $y^{\prime} \in \Lambda \cap \mathbb{B}_{\delta}(\bar{x})$, by the definition of the inner product on $\mathbb{R}^{2}$, we deduce

$$
\begin{aligned}
& \left\langle v-\left(y^{\prime}-\bar{x}\right), \bar{x}-x\right\rangle \\
& =\cos \angle\left(v-\left(y^{\prime}-\bar{x}\right), \bar{x}-x\right)\left\|v-\left(y^{\prime}-\bar{x}\right)\right\|\|\bar{x}-x\| \\
& \leq \epsilon\left\|v-\left(y^{\prime}-\bar{x}\right)\right\|\|\bar{x}-x\| \quad\left(\forall y^{\prime} \in \Lambda \cap \mathbb{B}_{\delta}(\bar{x})\right)\left(\forall(x, v) \in V_{\delta}\right),
\end{aligned}
$$

where

$$
V_{\delta}:=\left\{(x, v) \in \operatorname{gph} N_{\Omega}^{\text {prox }} \mid x+v \in \mathbb{B}_{\delta}(\bar{x}), x \in P_{\Omega}(x+v)\right\}
$$




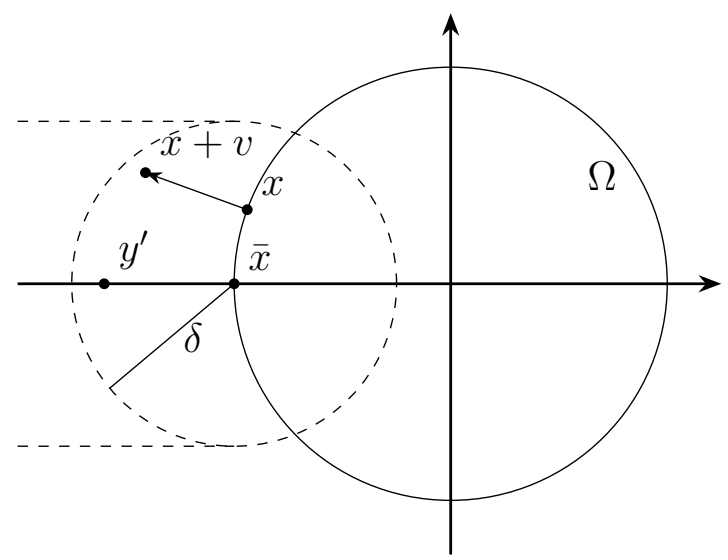

Figure 3.3: Illustration of the sets in Example 3.3.10.

which shows that $\Omega$ is $\epsilon$-super-regular at a distance relative to $P_{\Omega}^{-1}(\bar{x})$ at $\bar{x}$ for any $\epsilon \in(0,0.5)$. Similarly, the same is true for any $\epsilon>0.5$ when taking a ball with radius $\delta$ around $\bar{x}$, where $\delta<1$. Thus, $\Omega$ is super-regular at a distance relative to $P_{\Omega}^{-1}(\bar{x})$ at $\bar{x}$.

In fact, we can enlarge our neighborhood from a ball to a tube. Fix $\bar{x} \in \Omega, \epsilon>0$ and some $\delta \in(0,1)$ such that the above construction is satisfied. Then

$$
U:=\bigcup_{\substack{z \in P_{\Omega}^{-1}(\bar{x}) \\\|z\| \geq 1}} \mathbb{B}_{\delta}(z)
$$

is a neighborhood for $\bar{x}$ such that $\epsilon$-super-regularity relative to $\Lambda=P_{\Omega}^{-1}(\bar{x})$ is satisfied for $\Omega$. Fortunately, our violation $\epsilon$ is not worse compared to the neighborhood being a ball with radius $\delta$ around $\bar{x}$. This allows us to include more points in $\Lambda \cap U$ without violating (3.8).

Proposition 3.3.11 (characterization of super-regularity at a distance). The following hold:

(i) A nonempty set $\Omega \subset \mathcal{E}$ is $\epsilon$-super-regular at a distance relative to $\Lambda$ at $\bar{x}$ if and only if there is a neighborhood $U_{\epsilon}$ of $\bar{x}$ such that

$$
\begin{aligned}
\|x-y\|^{2} \leq \epsilon\left\|\left(y^{\prime}-y\right)-\left(x^{\prime}-x\right)\right\|\|x-y\|+ & \left\langle x^{\prime}-y^{\prime}, x-y\right\rangle \\
& \left(\forall y^{\prime} \in U_{\epsilon} \cap \Lambda\right)\left(\forall y \in P_{\Omega} y^{\prime}\right)
\end{aligned}
$$

with $x^{\prime}=x+v \in U_{\epsilon}$ for all $(x, v) \in V_{\epsilon}$ for $V_{\epsilon}$ defined by (3.11).

(ii) Let $\Omega \subset \mathcal{E}$ be $\epsilon$-super-regular at a distance relative to $\Lambda$ at $\bar{x}$. Then

$$
\|x-y\| \leq \epsilon\left\|\left(y^{\prime}-y\right)-\left(x^{\prime}-x\right)\right\|+\left\|x^{\prime}-y^{\prime}\right\| \quad\left(\forall y^{\prime} \in U_{\epsilon} \cap \Lambda\right)\left(\forall y \in P_{\Omega} y^{\prime}\right)
$$

with $x^{\prime}=x+v \in U_{\epsilon}$ for all $(x, v) \in V_{\epsilon}$. 
Proof. (i). Let $\Omega \subset \mathcal{E}$ be $\epsilon$-super-regular at a distance relative to $\Lambda \subset \mathcal{E}$ at $\bar{x}$. Then, for fixed $\epsilon>0$, there exists a neighborhood $U_{\epsilon}$ of $\bar{x}$ such that for all $(x, v) \in V_{\epsilon}$ for $V_{\epsilon}$ defined by (3.11) and $x^{\prime}=x+v \in U_{\epsilon}$ the following hold:

$$
\begin{aligned}
\|x-y\|^{2}=\langle x-y, x-y\rangle & =\left\langle y^{\prime}-y-\left(x^{\prime}-x\right), x-y\right\rangle+\left\langle x^{\prime}-y^{\prime}, x-y\right\rangle \\
& \leq \epsilon\left\|y^{\prime}-y-\left(x^{\prime}-x\right)\right\|\|x-y\|+\left\langle x^{\prime}-y^{\prime}, x-y\right\rangle .
\end{aligned}
$$

This proves the first part of the equivalence. For the remaining one let $U_{\epsilon}$ be a neighborhood of $\bar{x}$ such that

$$
\|x-y\|^{2} \leq \epsilon\left\|\left(y^{\prime}-y\right)-\left(x^{\prime}-x\right)\right\|\|x-y\|+\left\langle x^{\prime}-y^{\prime}, x-y\right\rangle \quad\left(\forall y^{\prime} \in U_{\epsilon} \cap \Lambda\right)\left(\forall y \in P_{\Omega}\left(y^{\prime}\right)\right)
$$

holds with $x^{\prime}=x+v \in U_{\epsilon}$ for all $(x, v) \in V_{\epsilon}$ for $V_{\epsilon}$ defined by (3.11). (3.14) is equivalent to

$\left\langle y^{\prime}-y-\left(x^{\prime}-x\right), x-y\right\rangle+\left\langle x^{\prime}-y^{\prime}, x-y\right\rangle \leq \epsilon\left\|\left(y^{\prime}-y\right)-\left(x^{\prime}-x\right)\right\|\|x-y\|+\left\langle x^{\prime}-y^{\prime}, x-y\right\rangle$,

$\left(\forall y^{\prime} \in U_{\epsilon} \cap \Lambda\right)\left(\forall y \in P_{\Omega}\left(y^{\prime}\right)\right)$ by the calculations made before. Subtracting $\left\langle x^{\prime}-y^{\prime}, x-y\right\rangle$ from both sides and inserting $v=x^{\prime}-x$ yields

$$
\left\langle y^{\prime}-y-v, x-y\right\rangle \leq \epsilon\left\|\left(y^{\prime}-y\right)-v\right\|\|x-y\|, \quad\left(\forall y^{\prime} \in U_{\epsilon} \cap \Lambda\right)\left(\forall y \in P_{\Omega}\left(y^{\prime}\right)\right) .
$$

Reordering the left-hand side we deduce the definition of $\epsilon$-super-regular for $\Omega$ at $\bar{x}$

$$
\left\langle v-\left(y^{\prime}-y\right), y-x\right\rangle \leq \epsilon\left\|\left(y^{\prime}-y\right)-v\right\|\|x-y\|, \quad\left(\forall y^{\prime} \in U_{\epsilon} \cap \Lambda\right)\left(\forall y \in P_{\Omega}\left(y^{\prime}\right)\right) .
$$

(ii). The second part follows from (i) by applying the Cauchy-Schwarz inequality to the vectors $x^{\prime}-y^{\prime}$ and $x-y$.

Definition 3.3.6 implies pointwise almost nonexpansivity of the projector and reflector on a neighborhood of a point $\bar{x}$ in $\Omega$ relative to points not in $\Omega$. This is of particular interest, since the fixed points of $T_{A, B}^{\lambda}$ (depending on $\lambda<1$ ) need not be in either of the sets $A$ and $B$ if the problem is inconsistent (see Theorem 4.2.3, where we do not require that $A \cap B \neq \emptyset)$.

Proposition 3.3.12 (regularity of projectors and reflectors at a distance). Let $\Omega \subset \mathcal{E}$ be nonempty and closed, and let $U$ be a neighborhood of $\bar{x} \in \Omega$. Let $\Lambda:=P_{\Omega}^{-1}(\bar{x}) \cap U$. If $\Omega$ is $\epsilon$-super-regular at a distance at $\bar{x}$ relative to $\Lambda$ with constant $\epsilon$ on the neighborhood $U$, then the following hold:

(i) If $\epsilon \in[0,1)$, then the projector $P_{\Omega}$ is pointwise almost nonexpansive at each $y^{\prime} \in \Lambda$ with violation $\tilde{\epsilon}$ on $U$ for $\tilde{\epsilon}:=4 \epsilon /(1-\epsilon)^{2}$. That is, at each $y^{\prime} \in \Lambda$

$$
\begin{aligned}
& \|x-y\| \leq \sqrt{1+\widetilde{\epsilon}}\left\|x^{\prime}-y^{\prime}\right\|=\frac{1+\epsilon}{1-\epsilon}\left\|x^{\prime}-y^{\prime}\right\| \\
& \qquad \quad\left(\forall x^{\prime} \in U\right)\left(\forall x \in P_{\Omega} x^{\prime}\right)\left(\forall y \in P_{\Omega} y^{\prime}\right) .
\end{aligned}
$$


(ii) If $\epsilon \in[0,1)$, then the projector $P_{\Omega}$ is pointwise almost firmly nonexpansive at each $y^{\prime} \in \Lambda$ with violation $\widetilde{\epsilon}_{2}$ on $U$ for $\widetilde{\epsilon}_{2}:=4 \epsilon(1+\epsilon) /(1-\epsilon)^{2}$. That is, at each $y^{\prime} \in \Lambda$

$$
\begin{aligned}
\|x-y\|^{2}+\left\|\left(x^{\prime}-x\right)-\left(y^{\prime}-y\right)\right\|^{2} \leq\left(1+\tilde{\epsilon}_{2}\right)\left\|x^{\prime}-y^{\prime}\right\| \\
\\
\left(\forall x^{\prime} \in U\right)\left(\forall x \in P_{\Omega} x^{\prime}\right)\left(\forall y \in P_{\Omega} y^{\prime}\right) .
\end{aligned}
$$

(iii) The reflector $R_{\Omega}$ is pointwise almost nonexpansive at each $y^{\prime} \in \Lambda$ with violation $\widetilde{\epsilon}_{3}:=8 \epsilon(1+\epsilon) /(1-\epsilon)^{2}$ on $U$. That is, for all $y^{\prime} \in \Lambda$

$$
\|x-y\| \leq \sqrt{1+\widetilde{\epsilon}_{3}}\left\|x^{\prime}-y^{\prime}\right\| \quad\left(\forall x^{\prime} \in U\right)\left(\forall x \in R_{\Omega} x^{\prime}\right)\left(\forall y \in R_{\Omega} y^{\prime}\right) .
$$

Proof. Our proof follows that of [105, Theorem 3.1]. Before proving each of the statements individually, note the following. Take any $x^{\prime} \in U$. Then for each $x \in P_{\Omega} x^{\prime}$ we have $\left(x, x^{\prime}-x\right) \in \operatorname{gph} N_{\Omega}^{\text {prox }} \subset \operatorname{gph} N_{\Omega}$. Moreover, by construction $\left(x, x^{\prime}-x\right) \in V_{\epsilon}$, where $V_{\epsilon}$ is defined by (3.11).

(i). By choosing $x^{\prime} \in U$ and $x \in P_{\Omega} x^{\prime}$ we get $\left(x, x^{\prime}-x\right) \in \operatorname{gph} N_{\Omega}^{\text {prox }} \subset \operatorname{gph} N_{\Omega}$. Applying Proposition 3.3.11(ii) yields

$$
\|y-x\| \leq \epsilon\left\|\left(x^{\prime}-x\right)-\left(y^{\prime}-y\right)\right\|+\left\|y^{\prime}-x^{\prime}\right\|,
$$

whenever $y^{\prime} \in U \cap \Lambda$ and $y \in P_{\Omega} y^{\prime}$. Exploiting the triangle inequality we deduce

$$
\|y-x\| \leq \epsilon\left(\left\|y^{\prime}-x^{\prime}\right\|+\|y-x\|\right)+\left\|y^{\prime}-x^{\prime}\right\|
$$

and thus conclude the claimed result.

(ii). By super-regularity at a distance relative to $\Lambda$ of $\Omega$ and Proposition 3.3.11(i) we have

$$
\begin{aligned}
& \|x-y\|^{2}+\left\|\left(x^{\prime}-x\right)-\left(y^{\prime}-y\right)\right\|^{2} \\
& =2\|x-y\|^{2}+\left\|x^{\prime}-y^{\prime}\right\|^{2}-2\left\langle x^{\prime}-y^{\prime}, x-y\right\rangle \\
& \leq\left\|x^{\prime}-y^{\prime}\right\|^{2}+2 \epsilon\left\|\left(x^{\prime}-x\right)-\left(y^{\prime}-y\right)\right\|\|x-y\|,
\end{aligned}
$$

for $\left(x, x^{\prime}-x\right) \in V_{\epsilon}$ and $y^{\prime} \in U \cap \Lambda, y \in P_{\Omega} y^{\prime}$. Together with the triangle inequality this implies

$$
\begin{aligned}
& \|x-y\|^{2}+\left\|\left(x^{\prime}-x\right)-\left(y^{\prime}-y\right)\right\|^{2} \\
& \leq\left\|x^{\prime}-y^{\prime}\right\|^{2}+2 \epsilon\left(\left\|x^{\prime}-y^{\prime}\right\|+\|x-y\|\right)\|x-y\| .
\end{aligned}
$$

Using part (i), we deduce

$$
\|x-y\|^{2}+\left\|\left(x^{\prime}-x\right)-\left(y^{\prime}-y\right)\right\|^{2} \leq\left(1+4 \epsilon \frac{1+\epsilon}{(1-\epsilon)^{2}}\right)\left\|x^{\prime}-y^{\prime}\right\|^{2}
$$


for all $\left(x, x^{\prime}-x\right) \in V_{\epsilon}$ and for all $y \in P_{\Omega} y^{\prime}$ at each $y^{\prime} \in U \cap \Lambda$. Since, as mentioned in the beginning, for all $x^{\prime} \in U$ it holds that $\left(x, x^{\prime}-x\right) \in V_{\epsilon}$ for all $x \in P_{\Omega} x^{\prime},(3.15)$ holds at each $y^{\prime} \in \Lambda=\Lambda \cap U$ for all $x \in P_{\Omega} x^{\prime}$ whenever $x^{\prime} \in U$. By Proposition 3.1.5 with $\alpha=1 / 2$, we conclude that $P_{\Omega}$ is pointwise almost firmly nonexpansive at each $y^{\prime} \in \Lambda$ with violation $4 \epsilon(1+\epsilon) /(1-\epsilon)^{2}$ on $U$.

(iii). By (ii), $P_{\Omega}$ is pointwise almost firmly nonexpansive at each $y^{\prime} \in \Lambda$ with violation $4 \epsilon(1+\epsilon) /(1-\epsilon)^{2}$ on $U$. Thus, by Proposition 3.1.5, the reflector, $R_{\Omega}:=$ $2 P_{\Omega}-\mathrm{Id}$, is pointwise almost nonexpansive at each $y^{\prime} \in \Lambda$ with violation

$$
8 \epsilon(1+\epsilon) /(1-\epsilon)^{2}
$$

on $U$.

Remark 3.3.13. By Remark 3.3.4, the statement in Proposition 3.3.12 is also true for convex and prox-regular sets (this can be deduced for instance from [105, Theorem 3.1]). Moreover, in the case of convex sets, the violation $\epsilon$ is 0 (compare this to Example 3.3.2(ii)). This result can be also found in [15, Proposition 4.8 and Corollary 4.10].

Remark 3.3.14. By Proposition 3.1.7, compositions of pointwise almost averaged mappings are again pointwise almost averaged. Proposition 3.3.12 yields that projectors and reflectors onto sets, that are super-regular at a distance, are pointwise almost averaged. Combining both results, suggests that compositions of projectors and reflectors onto sets, that are super-regular at a distance, are pointwise almost averaged. This observation will be important for our analysis of the relaxed Douglas-Rachford mapping in Chapter 4.

\subsection{Regularities of Collections of Sets}

In Theorem 3.2.6 there were two main ingredients for the quantitative convergence result. First, the mapping $T$ has to be almost averaged. As seen in Section 3.3, this is implied by the regularity of sets involved if the mapping $T$ is composed of projectors. The second assumption made in Theorem 3.2.6 was metric subregularity of $T-\mathrm{Id}$. This section is devoted to the investigation of this property in the context of feasibility problems.

Direct verification of metric subregularity is notoriously difficult. This is no different for the relaxed Douglas-Rachford or its cyclic version. In principle, one must show that the coderivative (the generalized Jacobian) of the $T$ mapping is injective on neighborhoods of Fix $T$ [55, Theorems 4B.1 and 4C.2]. Since the mappings we are interested in this thesis are based on projectors onto sets, another route is available for showing metric subregularity which uses characterizations of the regularity of sets in relation to each other. In the context of consistent set feasibility, metric subregularity of a particular set-valued mapping on the product space has been shown to be equivalent to subtransversality of the collection of sets [88]. This was 
expanded in [105, Definition 3.2] to account for inconsistent set feasibility. Luke, Thao and Teboulle showed in [104] that subtransversallity is indeed necessary for R-linear convergence of alternating projections for consistent feasibility. If the sets are convex, then subtransversality is even necessary and sufficient, i.e. equivalent to R-linear convergence [104, Theorem 8 and 9]. In Chapter 4 we will use the connection between subtransversality of the collection of sets also for the analysis of the relaxed Douglas-Rachford method. Note that this general definition can simplify in special cases such as intersecting sets as is discussed in Proposition 4.5.1.

Definition 3.4.1 (subtransversal collection of sets). Let $\left\{\Omega_{1}, \Omega_{2}, \ldots, \Omega_{m}\right\}$ be a collection of nonempty closed subsets of $\mathcal{E}$ and define $\Psi: \mathcal{E}^{m} \rightrightarrows \mathcal{E}^{m}$ by $\Psi(x):=$ $P_{\Omega}(\Pi x)-\Pi x$, where $\Omega:=\Omega_{1} \times \Omega_{2} \times \cdots \times \Omega_{m}$, the projection $P_{\Omega}$ is with respect to the Euclidean norm on $\mathcal{E}^{m}$ and $\Pi: x=\left(x_{1}, x_{2}, \ldots, x_{m}\right) \mapsto\left(x_{2}, x_{3}, \ldots, x_{m}, x_{1}\right)$ is the permutation mapping on the product space $\mathcal{E}^{m}$. Let $\bar{x}=\left(\bar{x}_{1}, \bar{x}_{2}, \ldots, \bar{x}_{m}\right) \in \mathcal{E}^{m}$ and $\bar{y} \in \Psi(\bar{x})$. The collection of sets is said to be subtransversal with constant $\kappa$ relative to $\Lambda \subset \mathcal{E}^{m}$ at $\bar{x}$ for $\bar{y}$ if $\Psi$ is metrically subregular at $\bar{x}$ for $\bar{y}$ on some neighborhood $U$ of $\bar{x}$ with constant $\kappa$ relative to $\Lambda$.

In contrast to the original model setting, where $\left\{\Omega_{1}, \Omega_{2}, \ldots, \Omega_{m}\right\}$ is a collection of subsets on $\mathcal{E}$, our definition of subtransversality is formulated on the product space $\mathcal{E}^{m}$ where $\Omega_{1} \times \Omega_{2} \times \cdots \times \Omega_{m} \subset \mathcal{E}^{m}$. Note that the original definition in [105] was formulated for gauges. The advantage of the more general formulation in [105] is to allow more generic concepts than only linear structures. Since we are, in the context of this thesis, interested in linear convergence, the version presented here is specialized to linear gauges. As it turns out, it will be useful that subtransversality is preserved, when we add sets to the considered collection of sets, that are the translates of the original sets.

Lemma 3.4.2 (subtransversality under addition). Let $\left\{\Omega_{1}, \Omega_{2} \ldots, \Omega_{m}\right\} \subset \mathcal{E}$ be a subtransversal collection of sets at a point $\bar{x}=\left(\bar{x}_{1}, \bar{x}_{2}, \ldots, \bar{x}_{m}\right)$ for $\bar{y} \in \Psi(\bar{x})$ relative to $\Lambda \subset \mathcal{E}^{m}$ with modulus $\kappa$. Then the collection

$$
\left\{\Omega_{1}, \Omega_{2} \ldots, \Omega_{m}, \Omega_{1}-g, \Omega_{2}-g, \ldots, \Omega_{m}-g\right\} \subset \mathcal{E}
$$

for some $g \in \mathcal{E}$ is subtransversal at

$$
\tilde{x}=\left(\bar{x}_{1}-g, \bar{x}_{2}, \bar{x}_{3}, \ldots, \bar{x}_{m}, \bar{x}_{1}, \bar{x}_{2}-g, \bar{x}_{3}-g, \ldots, \bar{x}_{m}-g\right) \in \mathcal{E}^{2 m}
$$

for

$$
\tilde{y}=(\bar{y}, \bar{y})=\left(\bar{y}_{1}, \bar{y}_{2}, \ldots, \bar{y}_{m}, \bar{y}_{1}, \bar{y}_{2}, \ldots, \bar{y}_{m}\right) \in \mathcal{E}^{2 m}
$$

relative to

$\tilde{\Lambda}=\left\{z \in \mathcal{E}^{2 m} \mid\left(z_{m+1}, z_{2}, z_{3}, \ldots, z_{m}\right) \in \Lambda,\left(z_{1}, z_{m+2}, z_{m+3}, \ldots, z_{2 m}\right) \in \Lambda-(g, \ldots, g)\right\}$

with modulus $\kappa$. 
Proof. We will show the result only for $m=2$ for reasons of simplicity and since one can easily enlarge the number of sets used in the proof by the same pattern shown here. For $s \in \mathbb{N}$ denote by $\Pi_{\mathcal{E}}^{s}$ the permutation mapping on $\mathcal{E}^{s}$.

Let $U \subset \mathcal{E}^{2}$ be a neighborhood of $\bar{x} \in \mathcal{E}^{2}$ such that subtransversality holds at $\bar{x}$ for $\bar{y}$ relative to $\Lambda$. Define $\Omega:=\Omega_{1} \times \Omega_{2}$ and therefore $\left(\Omega_{1}-g\right) \times\left(\Omega_{2}-g\right)=\Omega-(g, g)$. Likewise set

$$
\tilde{U}:=\left\{z \in \mathcal{E}^{4} \mid\left(z_{3}, z_{2}\right) \in U, z_{1}=z_{3}-g, z_{4}=z_{2}-g\right\}
$$

Thus every $z \in \tilde{U} \cap \tilde{\Lambda}$ can be expressed as $\left(x_{1}-g, x_{2}, x_{1}, x_{2}-g\right)^{T}$ for some $\left(x_{1}, x_{2}\right) \in$ $U \cap \Lambda$.

To show subtransversality of $\left\{\Omega_{1}, \Omega_{2}, \Omega_{1}-g, \Omega_{2}-g\right\}$ we have to verify metric subregularity of $\Psi=P_{\Omega}\left(\Pi_{\mathcal{E}}^{4}\right)-\Pi_{\mathcal{E}}^{4}$ for $\tilde{y} \in \Psi(\tilde{x})$ relative to $\tilde{\Lambda}$ on $\tilde{U}$, a neighborhood of $\tilde{x}$.

First, we show that $\tilde{y} \in \Psi(\tilde{x})$, i.e. $\tilde{y} \in P_{\Omega \times \Omega-(0,0, g, g)}\left(\Pi_{\mathcal{E}}^{4}(\tilde{x})\right)-\Pi_{\mathcal{E}}^{4}(\tilde{x})$. Let $\tilde{x}$ be defined by (3.16) then

$$
\begin{aligned}
P_{\Omega \times \Omega-(0,0, g, g)} & \left(\Pi_{\mathcal{E}}^{4}(\tilde{x})\right)-\Pi_{\mathcal{E}}^{4}(\tilde{x}) \\
= & \left(P_{\Omega}\left(\tilde{x}_{2}, \tilde{x}_{3}\right)-\left(\tilde{x}_{2}, \tilde{x}_{3}\right), P_{\Omega-(g, g)}\left(\tilde{x}_{4}, \tilde{x}_{1}\right)-\left(\tilde{x}_{4}, \tilde{x}_{1}\right)\right) \\
= & \left(P_{\Omega}\left(\bar{x}_{2}, \bar{x}_{1}\right)-\left(\bar{x}_{2}, \bar{x}_{1}\right), P_{\Omega-(g, g)}\left(\bar{x}_{2}-g, \bar{x}_{1}-g\right)-\left(\bar{x}_{2}-g, \bar{x}_{1}-g\right)\right) \\
= & \left(P_{\Omega}\left(\bar{x}_{2}, \bar{x}_{1}\right)-\left(\bar{x}_{2}, \bar{x}_{1}\right), P_{\Omega}\left(\bar{x}_{2}, \bar{x}_{1}\right)-\left(\bar{x}_{2}, \bar{x}_{1}\right)\right),
\end{aligned}
$$

where the last equality holds since $P_{C-g}(x-g)=P_{C}(x)-g$ for any set $C$. Then (3.17) yields

$$
\begin{aligned}
P_{\Omega \times \Omega-(0,0, g, g)} & \left(\Pi_{\mathcal{E}}^{4}(\tilde{x})\right)-\Pi_{\mathcal{E}}^{4}(\tilde{x}) \\
& =\left(P_{\Omega}\left(\bar{x}_{2}, \bar{x}_{1}\right)-\left(\bar{x}_{2}, \bar{x}_{1}\right), P_{\Omega}\left(\bar{x}_{2}, \bar{x}_{1}\right)-\left(\bar{x}_{2}, \bar{x}_{1}\right)\right) \\
& \ni(\bar{y}, \bar{y})=\tilde{y}
\end{aligned}
$$

since $\bar{y} \in P_{\Omega}\left(\Pi_{\mathcal{E}}^{2} \bar{x}\right)-\Pi_{\mathcal{E}}^{2} \bar{x}$ by the assumptions on subtransversality of $\left\{\Omega_{1}, \Omega_{2}, \ldots, \Omega_{m}\right\}$. By $\tilde{x} \in \tilde{\Lambda}$ this shows $\tilde{y} \in \Psi(\tilde{x})$ as claimed.

It remains to prove that inequality (3.4) holds for $\Psi$ and at $\tilde{x}$ for $\tilde{y} \in \Psi(\tilde{x})$ relative to $\tilde{\Lambda}$ on $\tilde{U}$. For this, take a $\left(x_{1}-g, x_{2}, x_{1}, x_{2}-g\right)^{T} \in \tilde{U} \cap \tilde{\Lambda}$, then:

$$
\begin{aligned}
& \kappa^{2} \operatorname{dist}^{2}\left(P_{\Omega \times \Omega-(0,0, g, g)}\left(\Pi_{\mathcal{E}}^{4}\left(\begin{array}{c}
x_{1}-g \\
x_{2} \\
x_{1} \\
x_{2}-g
\end{array}\right)\right)-\Pi_{\mathcal{E}}^{4}\left(\begin{array}{c}
x_{1}-g \\
x_{2} \\
x_{1} \\
x_{2}-g
\end{array}\right),\left(\begin{array}{c}
\bar{y}_{1} \\
\bar{y}_{2} \\
\bar{y}_{1} \\
\bar{y}_{2}
\end{array}\right)\right) \\
= & \kappa^{2}\left(\operatorname{dist}^{2}\left(P_{\Omega}\left(\begin{array}{c}
x_{2} \\
x_{1}
\end{array}\right)-\left(\begin{array}{c}
x_{2} \\
x_{1}
\end{array}\right),\left(\begin{array}{c}
\bar{y}_{1} \\
\bar{y}_{2}
\end{array}\right)\right)+\operatorname{dist}^{2}\left(P_{\Omega-(g, g)}\left(\begin{array}{c}
x_{2}-g \\
x_{1}-g
\end{array}\right)-\left(\begin{array}{c}
x_{2}-g \\
x_{1}-g
\end{array}\right),\left(\begin{array}{c}
\bar{y}_{1} \\
\bar{y}_{2}
\end{array}\right)\right)\right),
\end{aligned}
$$


by rewriting the distance on $\mathcal{E}^{4}$ in terms of the distance on $\mathcal{E}^{2}$. Using again that $P_{C-g}(x-g)=P_{C}(x)-g$ for an arbitrary set $C,(3.18)$ ends up as

$$
\begin{aligned}
& \kappa^{2}\left(\operatorname{dist}^{2}\left(P_{\Omega}\left(\Pi_{\mathcal{E}}^{2}\left(\begin{array}{c}
x_{1} \\
x_{2}
\end{array}\right)\right)-\Pi_{\mathcal{E}}^{2}\left(\begin{array}{c}
x_{1} \\
x_{2}
\end{array}\right),\left(\begin{array}{c}
\bar{y}_{1} \\
\bar{y}_{2}
\end{array}\right)\right)+\operatorname{dist}^{2}\left(P_{\Omega}\left(\begin{array}{c}
x_{2} \\
x_{1}
\end{array}\right)-\left(\begin{array}{c}
x_{2} \\
x_{1}
\end{array}\right),\left(\begin{array}{c}
\bar{y}_{1} \\
\bar{y}_{2}
\end{array}\right)\right)\right) \\
= & 2 \kappa^{2}\left(\operatorname{dist}^{2}\left(P_{\Omega}\left(\begin{array}{c}
x_{2} \\
x_{1}
\end{array}\right)-\left(\begin{array}{c}
x_{2} \\
x_{1}
\end{array}\right),\left(\begin{array}{c}
\bar{y}_{1} \\
\bar{y}_{2}
\end{array}\right)\right)\right) \\
\geq & 2 \operatorname{dist}^{2}\left(\left(\begin{array}{c}
x_{1} \\
x_{2}
\end{array}\right),\left(P_{\Omega}\left(\Pi_{\mathcal{E}}^{2}(\cdot)\right)-\Pi_{\mathcal{E}}^{2}(\cdot)\right)^{-1}\left(\begin{array}{c}
\bar{y}_{1} \\
\bar{y}_{2}
\end{array}\right)\right),
\end{aligned}
$$

where the last inequality holds by subtransversality of $\left\{\Omega_{1}, \Omega_{2}\right\}$ at $\left(\bar{x}_{1}, \bar{x}_{2}\right)$ for $\left(\bar{y}_{1}, \bar{y}_{2}\right)$ relative to $\Lambda$ with modulus $\kappa$ on $U$. Rewriting (3.19) in the distance on $\mathcal{E}^{4}$ yields

$$
\begin{aligned}
& \kappa^{2} \operatorname{dist}^{2}\left(P_{\Omega \times \Omega-(0,0, g, g)}\left(\Pi_{\mathcal{E}}^{4}\left(\begin{array}{c}
x_{1}-g \\
x_{2} \\
x_{1} \\
x_{2}-g
\end{array}\right)\right)-\Pi_{\mathcal{E}}^{4}\left(\begin{array}{c}
x_{1}-g \\
x_{2} \\
x_{1} \\
x_{2}-g
\end{array}\right),\left(\begin{array}{c}
\bar{y}_{1} \\
\bar{y}_{2} \\
\bar{y}_{1} \\
\bar{y}_{2}
\end{array}\right)\right) \\
& \geq \operatorname{dist}^{2}\left(\left(\begin{array}{c}
x_{1} \\
x_{2} \\
x_{1} \\
x_{2}
\end{array}\right),\left\{\left(\begin{array}{c}
z_{1} \\
z_{2} \\
z_{3} \\
z_{4}
\end{array}\right) \mid P_{\Omega}\left(\begin{array}{c}
z_{2} \\
z_{1}
\end{array}\right)-\left(\begin{array}{c}
z_{2} \\
z_{1}
\end{array}\right) \ni\left(\begin{array}{c}
\bar{y}_{1} \\
\bar{y}_{2}
\end{array}\right), P_{\Omega-(g, g)}\left(\begin{array}{c}
z_{4} \\
z_{3}
\end{array}\right)-\left(\begin{array}{c}
z_{4} \\
z_{3}
\end{array}\right) \ni\left(\begin{array}{c}
\bar{y}_{1} \\
\bar{y}_{2}
\end{array}\right)\right\}\right) \\
& =\operatorname{dist}^{2}\left(\left(\begin{array}{c}
x_{1} \\
x_{2} \\
x_{1} \\
x_{2}
\end{array}\right),\left\{\left(\begin{array}{c}
z_{1} \\
z_{2} \\
z_{3} \\
z_{4}
\end{array}\right) \mid P_{\Omega}\left(\begin{array}{c}
z_{2} \\
z_{1}
\end{array}\right)-\left(\begin{array}{c}
z_{2} \\
z_{1}
\end{array}\right) \ni\left(\begin{array}{c}
\bar{y}_{1} \\
\bar{y}_{2}
\end{array}\right), P_{\Omega-(g, g)}\left(\begin{array}{c}
z_{4} \\
z_{3}
\end{array}\right)-\left(\begin{array}{c}
z_{4} \\
z_{3}
\end{array}\right) \ni\left(\begin{array}{c}
\bar{y}_{1} \\
\bar{y}_{2}
\end{array}\right)\right\}\right) \\
& =\operatorname{dist}^{2}\left(\left(\begin{array}{c}
x_{1} \\
x_{2} \\
x_{1}-g \\
x_{2}-g
\end{array}\right),\left\{\left(\begin{array}{c}
z_{1} \\
z_{2} \\
z_{3} \\
z_{4}
\end{array}\right) \mid P_{\Omega \times \Omega-(0,0, g, g)}\left(\begin{array}{c}
z_{2} \\
z_{1} \\
z_{4} \\
z_{3}
\end{array}\right)-\left(\begin{array}{c}
z_{2} \\
z_{1} \\
z_{4} \\
z_{3}
\end{array}\right) \ni\left(\begin{array}{c}
\bar{y}_{1} \\
\bar{y}_{2} \\
\bar{y}_{1} \\
\bar{y}_{2}
\end{array}\right)\right\}\right) \\
& =\operatorname{dist}^{2}\left(\left(\begin{array}{c}
x_{1}-g \\
x_{2} \\
x_{1} \\
x_{2}-g
\end{array}\right),\left\{\left(\begin{array}{c}
z_{3} \\
z_{2} \\
z_{1} \\
z_{4}
\end{array}\right) \mid P_{\Omega \times \Omega-(0,0, g, g)}\left(\begin{array}{c}
z_{2} \\
z_{1} \\
z_{4} \\
z_{3}
\end{array}\right)-\left(\begin{array}{c}
z_{2} \\
z_{1} \\
z_{4} \\
z_{3}
\end{array}\right) \ni\left(\begin{array}{c}
\bar{y}_{1} \\
\bar{y}_{2} \\
\bar{y}_{1} \\
\bar{y}_{2}
\end{array}\right)\right\}\right) \\
& =\operatorname{dist}^{2}\left(\left(\begin{array}{c}
x_{1}-g \\
x_{2} \\
x_{1} \\
x_{2}-g
\end{array}\right),\left(P_{\Omega \times \Omega-(0,0, g, g)}\left(\Pi_{\mathcal{E}}^{4}(\cdot)\right)-\Pi_{\mathcal{E}}^{4}(\cdot)\right)^{-1}\left(\begin{array}{c}
\bar{y}_{1} \\
\bar{y}_{2} \\
\bar{y}_{1} \\
\bar{y}_{2}
\end{array}\right)\right) \text {, }
\end{aligned}
$$

where the last three steps were just rearranging the expression to get the claimed result. This completes the proof.

Remark 3.4.3. The points involved in Lemma 3.4.2 depend on the order of the sets involved appearing in $\Omega$. Note that the property of being subtransversal itself does not depend on any order of the sets. Of particular interest for our later analysis is the case of two sets $A$ and $B$, where we change the order on the product space, in comparison to Lemma 3.4.2, in the following way

$$
(B-g) \times(A-g) \times A \times B \subset \mathcal{E}^{4} .
$$

This is in contrast to the order $A \times B \times A-g \times B-g$ as used in Lemma 3.4.2. In the setting of (3.20), the points $\widetilde{x}$ and $\widetilde{y}$ as well as the set $\tilde{\Lambda}$ change to

$$
\begin{aligned}
& \widetilde{x}^{\prime}=\left(\bar{x}_{1}, \bar{x}_{1}-g, \bar{x}_{2}-g, \bar{x}_{2}\right) \\
& \widetilde{y}^{\prime}=\left(-\bar{y}_{1},-\bar{y}_{2}, \bar{y}_{1}, \bar{y}_{2}\right) \\
& \tilde{\Lambda}^{\prime}=\left\{z \in \mathcal{E}^{4} \mid\left(z_{3}, z_{4}\right) \in \Lambda,\left(z_{2}, z_{1}\right) \in \Lambda-(g, g)\right\} .
\end{aligned}
$$


That is, the collection $\{B-g, A-g, A, B\} \subset \mathcal{E}$ is subtransversal at $\tilde{x}^{\prime}$ for $\tilde{y}^{\prime}$ relative to $\tilde{\Lambda}^{\prime}$. Note that the negative part of $\widetilde{y}$ emerged from the changed order of $B$ and $A$ in comparison to Lemma 3.4.2. Since $\{B-g, A-g, A, B\}$ is a set of sets, we do not have to change the order of the sets appearing in comparison to Lemma 3.4.2. By definition there is no order. The only thing that changed was the order of the sets appearing in the product space $\mathcal{E}^{4}$. As a consequence the points $\tilde{x}$ and $\tilde{y}$ changed.

Remark 3.4.4 (historical remarks and other types of set-regularity). The notion of subtransversality routes back to the research area of differential geometry (see for instance [90]). The definition, that is used in this field, is transversality and was originally defined for manifolds. Loosely defined, an intersection of two smooth manifolds is transversal if it is again a smooth manifold. In finite dimensions, subtransversality can be formulated via tangent spaces (see for instance [93]) and is strongly connected to the Conical Hull Intersection Property (CHIP)(first mentioned in [46] by Chui, Deutsch and Ward). Transversality was shown to be one assumption that yields linear convergence of alternating projections on manifolds (see for instance [93]) for manifolds with nonempty intersection. The connection between transversality and metric regularity for alternating projections was established for instance in $[87,93]$. Similarly, the authors in [87] also presented the connection between subtransversality and metric subregularity for consistent feasibility. They were able to prove that subtransversality for the method of alternating projections in a consistent 2 -set feasibility problem is necessary for convergence.

In addition to the historical origin of subtransversality, there are plenty of other notions for the regularity of collection of sets. (Local) linear regularity, for instance, was first used by Bauschke and Borwein as a key assumption for linear convergence of cyclic projections onto closed and convex sets (see for instance [11]). In the context of consistent feasibility, this property is nothing more than subtransversality (see Proposition 4.5.1). Other notions of regularity of collections of sets include bounded (linear) regularity [11], the normal property and Jameson's property (for both see [8] and references therein). Linear regularity for closed convex sets implies CHIP [14, Theorem 3] (we can restrict this to bounded linear regularity). For a recent survey, we refer the reader to [87]. We consider transversality again in the context of subspaces in Section 4.7. 


\section{CHAPTER 4}

\section{Relaxed Douglas-RACHFord}

Having introduced several projection methods and basic concepts for their analysis, this chapter focuses on a specific iterative algorithm - the relaxed Douglas-Rachford method. As defined in Algorithm 2.3.5, the relaxed Douglas-Rachford method generates a sequences of iterates $\left(x^{k}\right)_{k \in \mathbb{N}}$ for an initial point $x^{0} \in \mathcal{E}$ and some parameter $\lambda \in(0,1)$ by

$$
x^{k+1} \in T_{A, B}^{\lambda} x^{k}:=\bigcup_{b \in P_{B} x^{k}}\left\{\frac{\lambda}{2}\left(R_{A}\left(2 b-x^{k}\right)+x^{k}\right)+(1-\lambda) b\right\} \quad(\forall k \in \mathbb{N}) .
$$

The aim of the subsequent analysis is not only to study convergence in general, but to develop a quantitative convergence result for the relaxed Douglas-Rachford method. By extending previous work by Luke [97,98] and Li and Pong [94], we focus on super-regular sets at a distance, as characterized in Definition 3.3.6. The analysis presented here is based on the framework established by Luke, Tam and Thao in [105], which we introduced in Section 3.2. Our basic convergence template, Theorem 3.2.6, requires two main properties of a mapping to generate an R-linear convergent sequence. The first of these is pointwise almost averagedness. We show in Theorem 4.1.2 that the relaxed Douglas-Rachford method does not only satisfy

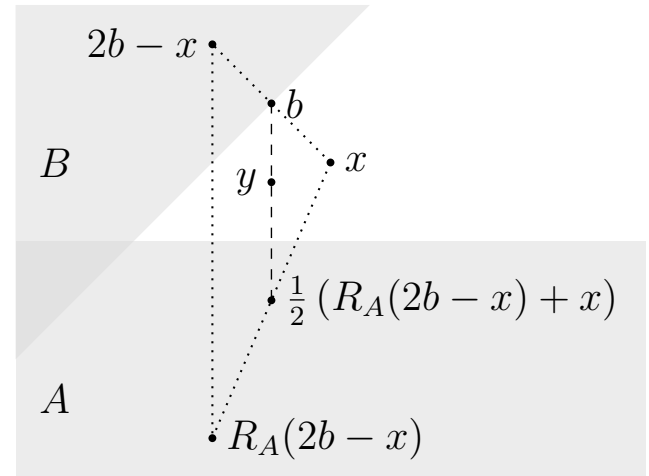

Figure 4.1: Illustration of applying $T_{A, B}^{\lambda}$ on a point $x$ yielding $y=T_{A, B}^{\lambda} x$ for a fixed $\lambda \in(0,1)$. 
this property, but that it is also single-valued at its fixed points. Analyzing the relaxed Douglas-Rachford method at its fixed points involves investigating these explicitly. In Section 4.2, we establish a characterization of the fixed point set. It depends only on single-valuedness of the mapping at these points, but not on the regularity of the set. The second property mentioned in Theorem 3.2.6, that we have to consider, is metric subregularity. This property on its own can be challenging to verify. For this reason, we introduced notions of regularity of collections of sets in Section 3.4 to serve as auxiliary properties for deducing metric subregularity. We devote Section 4.4 to the construction of a product space formulation that assists the actual proof of local convergence of the relaxed Douglas-Rachford method. The main result, Theorem 4.4.2, states in a nutshell that, if we have two closed super-regular sets with nonempty intersection, such that Fix $T_{A, B}^{\lambda} \neq \emptyset$, we can always find a suitable neighborhood on which the method converges $\mathrm{R}$ linearly. Beyond that, we are able to estimate the convergence rate depending on the chosen neighborhood. Applying this result to convex sets, we get global linear convergence (see Corollary 4.5.3). We present elementary examples in Section 4.6 to demonstrate the application of our result. We close this chapter by studying a special class of sets, namely subspaces. For this kind of regularity, the assumptions of our main result Theorem 4.4.2 are always satisfied. Moreover, we are able to reformulate our convergence result in terms of the so-called Friedrichs angle. Using an idea of Bauschke et al. [20], we improve the convergence rate for the method of relaxed Douglas-Rachford applied to subspaces.

Parts of the subsequent chapter can be found online in the preprint [101] by Luke and the present author.

\section{1. $T_{A, B}^{\lambda}$ IS Almost Averaged at Fix $T_{A, B}^{\lambda}$}

The relaxed Douglas-Rachford mapping $T_{A, B}^{\lambda}$ is a composition of projectors and reflectors. The almost averaging property is preserved under compositions of pointwise almost averaged mappings, as we have seen in Proposition 3.1.7. As an auxiliary statement for our main result of this section, Theorem 4.1.2, we start this section with the following lemma. Note that by $U_{\epsilon}(x)$ we denote a neighborhood of the point $x$ where the set, here $\Omega$, is $\epsilon$-super-regular at a distance with constant $\epsilon$.

Lemma 4.1.1. Let $\bar{x} \in \mathcal{E}$ and let $\Omega \subset \mathcal{E}$ be super-regular at a distance relative to $\Lambda \subset P_{\Omega}^{-1}(\bar{\omega})$ at $\bar{\omega}$, where $\bar{\omega} \in P_{\Omega} \bar{x}$ and $\bar{x} \in \Lambda$. In addition, for each $\epsilon>0$, let $\bar{x} \in U_{\epsilon}(\bar{\omega})$, where $U_{\epsilon}(\bar{\omega})$ is a neighborhood of $\bar{\omega}$ on which (3.8) holds. Then $P_{\Omega} \bar{x}=\{\bar{\omega}\}$.

Proof. For some fixed $\epsilon>0$, the assumptions of super-regularity at a distance of $\Omega$ relative to $\Lambda$ and Proposition 3.3.12(i) gives that there exists some neighborhood $U_{\epsilon}(\bar{\omega})$ such that $P_{\Omega}$ is pointwise almost nonexpansive at $\bar{x} \in \Lambda \cap U_{\epsilon}(\bar{\omega})$ on 
$U_{\epsilon}(\bar{\omega})$ with violation $\tilde{\epsilon}=4 \epsilon /(1-\epsilon)^{2}$. This implies single-valuedness of $P_{\Omega}$ at $\bar{x}$ by Proposition 3.1.9, i.e. that $\{\bar{\omega}\}=P_{\Omega} \bar{x}$, as claimed.

Theorem 4.1.2 ( $T_{A, B}^{\lambda}$ is pointwise almost firmly nonexpansive at its fixed points). Let $A, B$ be closed and nonempty, $\lambda \in(0,1)$ and $\bar{x} \in$ Fix $T_{A, B}^{\lambda} \neq \emptyset$. Let $\bar{b} \in P_{B} \bar{x}$ and $\bar{a} \in P_{A}(2 \bar{b}-\bar{x})$. Suppose that $B$ is super-regular at a distance relative to $\Lambda_{\bar{b}}:=$ $P_{B}^{-1}(\bar{b})$ at $\bar{b}$ and, likewise $A$ is super-regular at a distance relative to $\Lambda_{\bar{a}}:=P_{A}^{-1}(\bar{a})$ at $\bar{a}$. Suppose, moreover, that the following hold:

(i) For each $\epsilon>0, \bar{x} \in U_{\epsilon}(\bar{b})$, where $U_{\epsilon}(\bar{b})$ is a neighborhood of $\bar{b}$ on which (3.8) holds for $\epsilon$.

(ii) For each $\epsilon>0,2 \bar{b}-\bar{x} \in U_{\epsilon}(\bar{a})$, where $U_{\epsilon}(\bar{a})$ is a neighborhood of $\bar{a}$ on which (3.8) holds for $\epsilon$.

(iii) $R_{B}\left(\Lambda_{\bar{b}}\right) \subset \Lambda_{\bar{a}}$.

(iv) $R_{B}\left(U_{\epsilon}(\bar{b})\right) \subset U_{\epsilon}(\bar{a})$ for all $\epsilon>0$.

Then, $\{\bar{b}\}=P_{B} \bar{x},\{\bar{a}\}=P_{A} R_{B} \bar{x}, T_{A, B}^{\lambda}$ is single-valued at $\bar{x}$, and for all $\epsilon>0$ there exists a neighborhood $U(B, \epsilon, \bar{x})$ of $\bar{b}$ such that $T_{A, B}^{\lambda}$ is pointwise almost firmly nonexpansive at $\bar{x}$ with violation at most $\epsilon$ on $U(B, \epsilon, \bar{x})$.

Before we begin the proof of this statement, we would like to point out an important feature of our construction. The claimed pointwise almost averagedness of $T_{A, B}^{\lambda}$ at $\bar{x}$ holds on open subsets containing both $\bar{x}$ and $\bar{b}=P_{B} \bar{x}$. This follows from assumption (i). The conclusion of the theorem could have been equivalently stated: for all $\epsilon>0$ there exists a neighborhood $U(B, \epsilon, \bar{x})$ of $\bar{x}$ such that $T_{A, B}^{\lambda}$ is pointwise almost averaged at $\bar{x}$ with violation at most $\epsilon$ on $U$. We have presented the statement in terms of a neighborhood $U(B, \epsilon, \bar{x})$ containing $\bar{b}$ to emphasize the fact that the open sets on which the regularity of $T_{A, B}^{\lambda}$ holds is constructed relative to points $\bar{b}$ at a distance from the point of interest $\bar{x} \in$ Fix $T_{A, B}^{\lambda}$. The usual use of balls for neighborhoods is not convenient or appropriate for this setting.

Proof of Theorem 4.1.2. Under assumptions (i) and (ii), Lemma 4.1.1 yields $\{\bar{b}\}=$ $P_{B} \bar{x}$ and $\{\bar{a}\}=P_{A} R_{B} \bar{x}$, as claimed. From this, one can immediately conclude that $T_{A, B}^{\lambda}$ is single-valued at $\bar{x}$.

For any fixed $\epsilon_{B}>0$, we get by the assumptions on super-regularity at a distance of $B$ relative to $\Lambda_{b}$ and Proposition 3.3.12(ii) that there exists some neighborhood $U_{\epsilon_{B}}(\bar{b})$ such that $P_{B}$ is pointwise almost firmly nonexpansive at $\bar{x} \in \Lambda_{\bar{b}} \cap U\left(B, \epsilon_{B}, \bar{x}\right)$ on $U_{\epsilon_{B}}(\bar{b})$ with violation $\epsilon_{P_{B}}=4 \epsilon_{B}\left(1+\epsilon_{B}\right) /\left(1-\epsilon_{B}\right)^{2}$. Note that this also shows that $P_{B}$ is pointwise almost nonexpansive at $\bar{x}$ on $U_{\epsilon_{B}}(\bar{b})$. Similarly, by Proposition 3.3.12(iii), $R_{B}$ is pointwise almost nonexpansive at $\bar{x}$ with violation $\epsilon_{R_{B}}=$ $8 \epsilon_{B}\left(1+\epsilon_{B}\right) /\left(1-\epsilon_{B}\right)^{2}$ on $U_{\epsilon_{B}}(\bar{b})$. Likewise, for any $\epsilon_{A}>0$ there exists a neighborhood $U_{\epsilon_{A}}(\bar{a})$ of $\bar{a}$ such that $R_{A}$ is pointwise almost nonexpansive at $\bar{a}=2 \bar{b}-\bar{x}$ with violation $\epsilon_{R_{A}}=8 \epsilon_{A}\left(1+\epsilon_{A}\right) /\left(1-\epsilon_{A}\right)^{2}$ on $U_{\epsilon_{A}}(\bar{a})$. 
By (iii) and (iv), the assumptions of Proposition 3.1.7(ii) are satisfied. Hence, we deduce that for any fixed $\epsilon_{A}>0$ there exists a neighborhood $U\left(A, \epsilon_{R_{A} R_{B}}, \bar{x}\right)$ such that $R_{A} R_{B}$ is pointwise almost nonexpansive at $\bar{x}$ with violation at most

$$
\epsilon_{R_{A} R_{B}}=\epsilon_{R_{A}}+\epsilon_{R_{B}}+\epsilon_{R_{A}} \epsilon_{R_{B}}
$$

on $U(B, \epsilon, \bar{x})$.

By Proposition 3.1.5(ii) we get that $1 / 2\left(R_{A} R_{B}+\right.$ Id) is almost firmly nonexpansive at $\bar{x}$ with violation $\epsilon_{R_{A} R_{B}} / 2$ on $U_{\epsilon_{B}}(\bar{b})$. Again applying Proposition 3.1 .7 (i) yields pointwise almost nonexpansivity of $T_{A, B}^{\lambda}$ at $\bar{x}$ on $U_{\epsilon_{B}}(\bar{b})$ with violation at most

$$
\epsilon^{\prime}=\lambda(1 / 2) \epsilon_{R_{A} R_{B}}+(1-\lambda) \epsilon_{P_{B}} .
$$

Since the above properties hold for each $\epsilon_{B}>0$ and $\epsilon_{A}>0$, we can construct the neighborhoods above, given any $\epsilon>0$, so that $\epsilon^{\prime} \leq \epsilon$. We conclude that for any $\epsilon>0$ there is a neighborhood $U(B, \epsilon, \bar{x})$ such that $T_{A, B}^{\lambda}$ is pointwise almost nonexpansive at $\bar{x}$ on $U(B, \epsilon, \bar{x})$ with violation at most $\epsilon$. The corresponding neighborhood $U\left(A, \epsilon_{R_{A} R_{B}}, \bar{x}\right)$ of $\bar{a}$ will be denoted by $U(A, \epsilon, \bar{x})$. This completes the proof.

Corollary 4.1.3. In the setting of Theorem 4.1.2, fix $\bar{\epsilon}>0$ and let $U(B, \bar{\epsilon}, \bar{x})$ and $U(A, \bar{\epsilon}, \bar{x})$ be neighborhoods that satisfy the assumptions (i), (ii) and (iv) such that $T_{A, B}^{\lambda}$ is pointwise almost firmly nonexpansive at $\bar{x}$ with violation $\bar{\epsilon}$ on $U(B, \bar{\epsilon}, \bar{x})$. Then, for all $\epsilon<\bar{\epsilon}$ there exists a neighborhood $U(B, \epsilon, \bar{x})$ and a neighborhood $U(A, \epsilon, \bar{x})$ such that conditions (i), (ii) and (iv) hold in addition to the inclusions $U(A, \epsilon, \bar{x}) \subset U(A, \bar{\epsilon}, \bar{x})$ and $U(B, \epsilon, \bar{x}) \subset U(B, \bar{\epsilon}, \bar{x})$.

Corollary 4.1.3 implies that $T_{A, B}^{\lambda}$ is pointwise almost firmly nonexpansive at $\bar{x}$ with violation $\epsilon$ on $U(B, \epsilon, \bar{x})$. The strength of Corollary 4.1.3, however, is hidden in the proof given below and the explicit construction of the neighborhoods $U(B, \epsilon, \bar{x})$ and $U(A, \epsilon, \bar{x})$. Thus, under the assumptions of Theorem 4.1.2, and given the neighborhoods for some fixed violation $\bar{\epsilon}$, we are always able to restrict these neighborhoods to smaller sets where (3.8) holds with some violation smaller than $\bar{\epsilon}$.

Proof of Corollary 4.1.3. Our approach to prove this statement is based on an explicit construction of the neighborhoods $U(A, \epsilon, \bar{x})$ and $U(B, \epsilon, \bar{x})$.

Let $\epsilon<\bar{\epsilon}$. Then Theorem 4.1.2(i) implies that there exists a neighborhood $U(B, \epsilon, \bar{x})$ of $\bar{b}$ where (3.8) holds such that $U(B, \epsilon, \bar{x}) \subset U(B, \bar{\epsilon}, \bar{x})$. To see this, note that by (i) the existence of $U(B, \epsilon, \bar{x})$ is guaranteed and thus only $U(B, \epsilon, \bar{x}) \subset$ $U(B, \bar{\epsilon}, \bar{x})$ has to be proven. Let $\tilde{U}(B, \epsilon, \bar{x})$ be a neighborhood for $\epsilon$ where (3.8) holds. Then (3.8) is satisfied for $\bar{\epsilon}$ as well. Thus, $U(B, \epsilon, \bar{x}):=\tilde{U}(B, \epsilon, \bar{x}) \cap U(B, \bar{\epsilon}, \bar{x})$ is a neighborhood of $\bar{b}$ where Theorem 4.1.2(iv) holds and (3.8) is satisfied for both $\epsilon$ and $\bar{\epsilon}$, which shows that $U(B, \epsilon, \bar{x}) \subset U(B, \bar{\epsilon}, \bar{x})$ as required. Next, applying the reflection onto $B$ on both of neighborhoods $U(B, \epsilon, \bar{x})$ and $U(B, \bar{\epsilon}, \bar{x})$ yields

$$
R_{B}(U(B, \epsilon, \bar{x})) \subset R_{B}(U(B, \bar{\epsilon}, \bar{x})) .
$$


Let $\tilde{U}(A, \epsilon, \bar{x})$ be a neighborhood of $\bar{a}$ where (3.8) holds for $\epsilon$ such that Theorem 4.1.2(iv) is satisfied. That is

$$
R_{B}(U(B, \epsilon, \bar{x})) \subset \tilde{U}(A, \epsilon, \bar{x}) .
$$

Combining Theorem 4.1.2(iv) for the neighborhoods $U(B, \bar{\epsilon}, \bar{x})$ and $U(A, \bar{\epsilon}, \bar{x})$ and (4.1) we deduce

$$
R_{B}(U(B, \epsilon, \bar{x})) \subset R_{B}(U(B, \bar{\epsilon}, \bar{x})) \subset U(A, \bar{\epsilon}, \bar{x}) .
$$

This and (4.2) imply that

$$
R_{B}(U(B, \epsilon, \bar{x})) \subset \tilde{U}(A, \epsilon, \bar{x}) \cap U(A, \bar{\epsilon}, \bar{x}) .
$$

Set

$$
U(A, \epsilon, \bar{x}):=\tilde{U}(A, \epsilon, \bar{x}) \cap U(A, \bar{\epsilon}, \bar{x}) .
$$

Then, $U(A, \epsilon, \bar{x})$ is a neighborhood of $\bar{a}$ where (3.8) holds with $\epsilon$, since it is a subset of $\tilde{U}(A, \epsilon, \bar{x})$. Moreover, $U(B, \epsilon, \bar{x})$ and $U(A, \epsilon, \bar{x})$ satisfy Theorem 4.1.2(iv) by (4.4). By the construction of $U(A, \bar{\epsilon}, \bar{x})$ and the choice of $U(B, \epsilon, \bar{x})$ both sets satisfy the inclusions $U(A, \epsilon, \bar{x}) \subset U(A, \bar{\epsilon}, \bar{x})$ and $U(B, \epsilon, \bar{x}) \subset U(B, \bar{\epsilon}, \bar{x})$. This completes the proof.

Example 4.1.4. The following three examples illustrate the assumptions of Theorem 4.1.2. For these examples it is easy to determine the sets of fixed points of the mapping $T_{A, B}^{\lambda}$. In Theorem 4.2 .3 we give a precise characterization of Fix $T_{A, B}^{\lambda}$. More intuitively, the fixed points must lie on lines containing local best approximation points between the sets.

(i) (convex sets with empty intersection). Let $A$ and $B$ be closed convex subsets of $\mathcal{E}$. By Proposition 3.3.9 both sets are super-regular relative to $\mathcal{E}$ at any of their points, i.e. $\epsilon$-super-regular for all $\epsilon>0$. In fact, the violation can be set to 0 . Thus, as long as Fix $T_{A, B}^{\lambda} \neq \emptyset$ the mappings $P_{B}, R_{B}$ and $R_{A}$ are nonexpansive (i.e. no violation) at $\bar{x}$ on the whole space $\mathcal{E}$ by Proposition 3.3.12. Moreover, we can apply Theorem 4.1.2 to conclude that $T_{A, B}^{\lambda}$ is firmly nonexpansive at $\bar{x}$ on the neighborhood $\mathcal{E}$. For instance, consider the two sets

$$
\begin{aligned}
& A:=\left\{x=\left(x_{1}, x_{2}\right) \in \mathbb{R}^{2} \mid x_{1}^{2}+x_{2}^{2} \leq 1\right\}, \\
& B:=\left\{x=\left(x_{1}, x_{2}\right) \in \mathbb{R}^{2} \mid\left(x_{1}-3\right)^{2}+x_{2}^{2} \leq 1\right\} .
\end{aligned}
$$

The set of fixed points is given by the unique point

$$
\text { Fix } T_{A, B}^{\lambda}=\{\bar{x}\}=\left\{(2,0)-\frac{\lambda}{1-\lambda}(1,0)\right\}
$$

for fixed $\lambda \in(0,1)$. Moreover, we know by the above discussion that $T_{A, B}^{\lambda}$ is nonexpansive, since it is composed of projections onto these sets. 


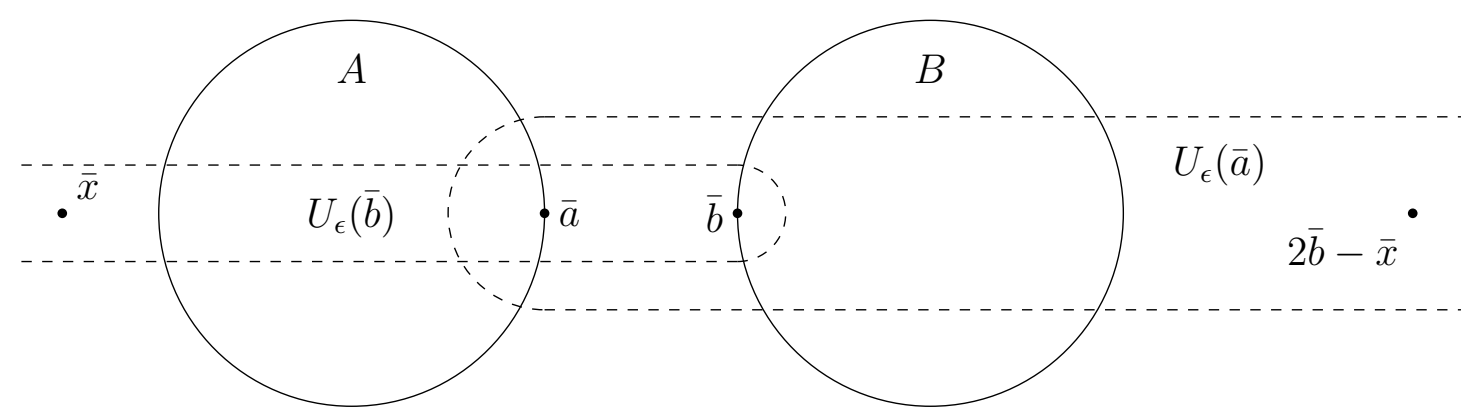

Figure 4.2: Illustration of a possible choice of neighborhoods in Example 4.1.4(ii).

(ii) (super-regular sets with empty intersection). Continuing with the concrete example above, suppose that $A$ and $B$ are spheres instead of balls,

$$
\begin{aligned}
& A:=\left\{x=\left(x_{1}, x_{2}\right) \in \mathbb{R}^{2} \mid x_{1}^{2}+x_{2}^{2}=1\right\} \\
& B:=\left\{x=\left(x_{1}, x_{2}\right) \in \mathbb{R}^{2} \mid\left(x_{1}-3\right)^{2}+x_{2}^{2}=1\right\} .
\end{aligned}
$$

The sets $A$ and $B$ are both nonconvex, but still super-regular. The set of fixed points is again given by the unique point

$$
\text { Fix } T_{A, B}^{\lambda}=\{\bar{x}\}=\left\{(2,0)-\frac{\lambda}{1-\lambda}(1,0)\right\}
$$

for fixed $\lambda \in(0,1)$. As seen in Example 3.3.10 both sets are super regular relative to radial directions. Thus, applying Theorem 4.1.2, we deduce that $T_{A, B}^{\lambda}$ for some fixed $\lambda \in(0,1)$ is only almost firmly nonexpansive at $\bar{x}$ on some neighborhood $U$. As noted before in Example 3.3.10, the neighborhood should be rather chosen as a tube than the more conventional ball. Such a choice of neighborhoods is visualized in Fig. 4.2

(iii) (super-regular sets with nonempty intersection). Next we translate the sets in (ii) such that they have exactly one common point in their intersection.

$$
\begin{aligned}
& A:=\left\{x=\left(x_{1}, x_{2}\right) \in \mathbb{R}^{2} \mid x_{1}^{2}+x_{2}^{2}=1\right\} \\
& B:=\left\{x=\left(x_{1}, x_{2}\right) \in \mathbb{R}^{2} \mid\left(x_{1}-2\right)^{2}+x_{2}^{2}=1\right\}
\end{aligned}
$$

The fixed point set then reduces to Fix $T_{A, B}^{\lambda}=\{(1,0)\}=A \cap B$. By (ii) we know that the assumptions of Theorem 4.1.2 are satisfied. In contrast to (ii), the fixed point is in the intersection of both sets. Thus, balls as neighborhoods are enough to get pointwise almost firm nonexpansivity. We do not need tubes or other constructions of a neighborhood to include points from a distance.

The examples show, that in case of closed balls and circles the assumptions are easily satisfied. Nonetheless, one has to take care of choosing neighborhoods in the right way to get a desired violation. 

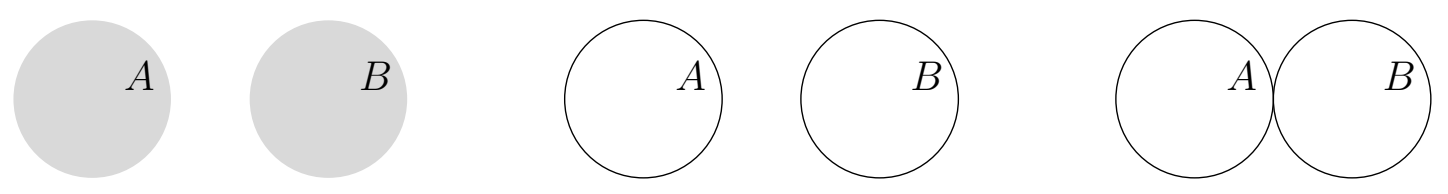

Figure 4.3: Illustration of the sets in Example 4.1.4(i)-(iii).

Example 4.1.4(i) yields the following specialization of Theorem 4.1.2.

Corollary 4.1.5. Let $\lambda \in(0,1)$ and Fix $T_{A, B}^{\lambda} \neq \emptyset$. If $A$ and $B$ are closed and convex, then $T_{A, B}^{\lambda}$ is firmly nonexpansive on $\mathcal{E}$.

Proof. Since $A$ and $B$ are both convex, one has by Proposition 3.3.9 that both sets are super-regular at a distance relative to $\mathcal{E}$ at any of their points. Applying Theorem 4.1.2, we deduce firm nonexpansivity of $T_{A, B}^{\lambda}$ since the violation $\epsilon$ can be set to 0, as seen in the proof of Proposition 3.3.9.

\subsection{Characterization of Fix $T_{A, B}^{\lambda}$}

We collect some facts and identities that will be useful throughout.

Lemma 4.2.1. Let $A$ and $B$ be closed and $T_{A, B}^{\lambda}$ defined as in Algorithm 2.3.5 with $\lambda \in(0,1)$. Let $x \in$ Fix $T_{A, B}^{\lambda} \neq \emptyset$ such that $T_{A, B}^{\lambda}$ is single-valued at $x$. Take $f \in P_{B} x$ and $y:=x-f$. Then, the following hold:

(i) $P_{B} x=\{f\}$, that is, $P_{B}$ is single-valued on Fix $T_{A, B}^{\lambda}$,

(ii) $P_{A}\left(R_{B} x\right)$ is single-valued, hence so is $R_{A}\left(R_{B} x\right)$,

(iii) $P_{A}(2 f-x)=P_{A} R_{B} x$,

(iv) $T_{A, B} x-x=P_{A} R_{B} x-P_{B} x$, where $T_{A, B}$ is the Douglas-Rachford mapping defined in Algorithm 2.3.4.

(v) $f+\frac{1-\lambda}{\lambda} y=P_{A}(2 f-x)$,

(vi) If $A$ is convex, then, for $e=P_{A} f$

$$
P_{A}\left(e+\frac{1}{1-\lambda}(f-e)\right)=e .
$$

Proof. (i)-(ii). Since

$$
T_{A, B}^{\lambda} x=\bigcup_{b \in P_{B} x^{k}}\left\{\frac{\lambda}{2}\left(R_{A}\left(2 b-x^{k}\right)+x^{k}\right)+(1-\lambda) b\right\},
$$

is just a single point, we conclude that $P_{B} x$ is a single point as well. This implies that $P_{A}\left(R_{B} x\right)$ and $R_{A}\left(R_{B} x\right)$ have to be single-valued too, as claimed. 
(iii). This is an easy implication of the single-valuedness of $P_{B}$ at $x$ :

$$
P_{A}(2 f-x)=P_{A}\left(2 P_{B} x-x\right)=P_{A}\left(R_{B} x\right) .
$$

(iv). This follows as well from the single-valuedness of $P_{B}$ at $x$ :

$$
\begin{aligned}
T_{A, B} x-x & =\frac{1}{2}\left(R_{A}\left(R_{B} x\right)+x\right)-x \\
& =\frac{1}{2}\left(R_{A}\left(R_{B} x\right)\right)-\frac{1}{2} x \\
& =P_{A}\left(R_{B} x\right)-\frac{1}{2} R_{B} x-\frac{1}{2} x \\
& =P_{A}\left(R_{B} x\right)-P_{B} x .
\end{aligned}
$$

(v). To see this, note that

$$
\begin{aligned}
& x=T_{A, B}^{\lambda} x=\frac{\lambda}{2}\left(R_{A}\left(R_{B} x\right)+x\right)+(1-\lambda) P_{B} x \\
& \Longleftrightarrow \quad(1-\lambda) x=\lambda\left(T_{D R} x-x\right)+(1-\lambda) P_{B} x \\
& \Longleftrightarrow \quad(1-\lambda)\left(x-P_{B} x\right)=\lambda\left(P_{A} R_{B} x-P_{B} x\right) \text {, }
\end{aligned}
$$

by (iv). Hence, with $f=P_{B} x$, this yields

$$
(1-\lambda)(x-f)=\lambda\left(P_{A}(2 f-x)-f\right) \Longleftrightarrow f+\frac{1-\lambda}{\lambda} y=P_{A}(2 f-x),
$$

by the definition of $y$.

(vi). This follows from the fact that $f-e \in N_{A}^{\text {prox }}(e)$. Since $A$ is convex, then all points in $e+N_{A}^{\text {prox }}(e)$ project back to $e$.

Remark 4.2.2. Note that (i) and (ii) of Lemma 4.2.1 together at some point $x \in \mathcal{E}$ are equivalent to the single-valuedness of $T_{A, B}^{\lambda}$ at $x$.

Theorem 4.2.3 (fixed points of relaxed DR). Let $A, B \subset \mathcal{E}$ both be closed and let $\lambda \in(0,1)$. Let $T_{A, B}^{\lambda}$ be single-valued at its fixed points on an open set $U \subset \mathcal{E}$. Then

$$
\operatorname{Fix} T_{A, B}^{\lambda} \cap U \subset \mathcal{M}
$$

where

$$
\mathcal{M}:=\left\{f-\frac{\lambda}{1-\lambda}(f-e) \mid f \in P_{B}\left(f-\frac{\lambda}{1-\lambda}(f-e)\right), \text { and } e \in P_{A} f\right\} \cap U .
$$

The inclusion is tight if $e \in P_{A}\left(f+\frac{\lambda}{1-\lambda}(f-e)\right)$ is true for the right-hand side. 
Proof. Let $x \in$ Fix $T_{A, B}^{\lambda} \cap U$. By the assumptions $T_{A, B}^{\lambda}$ is single-valued at $x$, and hence the results in Lemma 4.2.1 can be applied. As before denote by $f$ the projection $P_{B}(x)$. Reformulating Lemma 4.2.1(v) yields the desired form of the fixed point $x$.

$$
\begin{array}{rlrl}
x \in \mathrm{Fix} T_{A, B}^{\lambda} & \Longleftrightarrow & f+\frac{1-\lambda}{\lambda} y=P_{A}(2 f-x) \\
& \Longleftrightarrow f+\frac{1-\lambda}{\lambda}(x-f)=P_{A}(2 f-x) \\
& \Longleftrightarrow & x & =\frac{\lambda}{1-\lambda} P_{A}(2 f-x)-\frac{2 \lambda-1}{1-\lambda} f \\
& \Longleftrightarrow & x & =f-\frac{\lambda}{1-\lambda}\left(f-P_{A}(2 f-x)\right)
\end{array}
$$

By comparing with (4.6), we have to show that $P_{A} f=P_{A}(2 f-x)$.

Using this, then (4.7) becomes

$$
x=f-\frac{\lambda}{1-\lambda}\left(f-P_{A} f\right) .
$$

Finally, (4.6) follows from the fact that $f=P_{B} x$, since $x$ is a fixed point.

It remains to show that the inclusion is in fact an equality when

$$
e \in P_{A}\left(f+\frac{\lambda}{1-\lambda}(f-e)\right)
$$

for $e \in P_{A} f$. To see this, let $\widetilde{x} \in \mathcal{M} \cap U$ in (4.6). Then $\widetilde{x}:=f-\frac{\lambda}{1-\lambda}(f-e)$ for some $e \in P_{A} f$ and $f \in P_{B} \tilde{x}$ and

$$
\begin{aligned}
\widetilde{x}-T_{A, B}^{\lambda} \widetilde{x} & =\widetilde{x}-\frac{\lambda}{2}\left(R_{A} R_{B} \widetilde{x}+\widetilde{x}\right)-(1-\lambda) P_{B} \widetilde{x} \\
& =\lambda \widetilde{x}-\frac{\lambda}{2}\left(2 P_{A} R_{B} \widetilde{x}-2 P_{B} \widetilde{x}+2 \widetilde{x}\right)+(1-\lambda)\left(\widetilde{x}-P_{B} \widetilde{x}\right) \\
& =-\lambda\left(P_{A} R_{B} \widetilde{x}-P_{B} \widetilde{x}\right)+(1-\lambda)\left(\widetilde{x}-P_{B} \widetilde{x}\right) \\
& \ni-\lambda\left(P_{A} R_{B} \widetilde{x}-f\right)+(1-\lambda)(\widetilde{x}-f) \\
& =-\lambda\left(P_{A} R_{B} \widetilde{x}-f\right)-\lambda(f-e) \\
& =-\lambda\left(P_{A} R_{B} \widetilde{x}\right)+\lambda e .
\end{aligned}
$$

Thus $0 \in \widetilde{x}-T_{A, B}^{\lambda} \widetilde{x}$ if and only if $e \in P_{A} R_{B} \widetilde{x}$, which is equivalent to

$$
e \in P_{A}\left(f+\frac{\lambda}{1-\lambda}(f-e)\right)
$$

This concludes the proof. 
Remark 4.2.4. (i) Note that $f+\frac{\lambda}{1-\lambda}(f-e)=e+\frac{1}{1-\lambda}(f-e)$, so that for any $e \in P_{A} f, f-e$ is in the normal cone to $A$ at $e$. It follows immediately that, if $A$ is convex, then $P_{A}\left(e+\frac{1}{1-\lambda}(f-e)\right)=e$ for all $\lambda \in(0,1)$ and, by Theorem 4.2.3 the inclusion (4.6) is in fact equality for all $\lambda$ for which $f \in P_{B}\left(f-\frac{\lambda}{1-\lambda}(f-e)\right)$. Compare this to the statement in [98, Lemma $3.8]$, where the tight fixed point characterization holds for $\lambda \in[0,1 / 2]$. This is due to a slightly different characterization. The statement in [98], that $f$ is a local best approximation point, is actually incorrect. Where our description includes $f \in P_{B}\left(f-\frac{\lambda}{1-\lambda}(f-e)\right)$, and $e \in P_{A} f$, the version in [98, Lemma 3.8] states that $f$ is a local best approximation point [98, Definition 3.3]. Instead, what is needed to correct the statement is $f \in P_{B} P_{A} f$, and such a point needs not be a local best approximation point. To see this, consider a unit circle in $\mathbb{R}^{2}$ centered at the origin and a horizontal line passing through the point $(0,3 / 4)$. For the fixed point mapping $T_{A, B}^{\lambda}$ with $A$ the line and $B$ the circle, the point $\left(0,1-\frac{\lambda}{4(1-\lambda)}\right)$ is a fixed point for all $\lambda \in(0,4 / 5)$. However, the corresponding points $f=(0,1)$ and $e=(0,3 / 4)$ are not local best approximation points.

(ii) The condition $e \in P_{A}\left(f+\frac{\lambda}{1-\lambda}(f-e)\right)$ is easier to interpret with the identity $f+\frac{\lambda}{1-\lambda}(f-e)=e+\frac{1}{1-\lambda}(f-e)$. As $\lambda \nearrow 1$ this vector receeds from $A$ in the direction normal to $A$ at $e$. The larger the neighborhood on which the projection onto $A$ exists and is single-valued, the larger $\lambda$ can be before $e \notin$ $P_{A}\left(f+\frac{\lambda}{1-\lambda}(f-e)\right)$. If $A$ is convex, then $\lambda$ can be arbitrarily close to 1 . Still, $\lambda$ may need to be bounded away from 1 in order to ensure the other condition in the fixed point characterization (4.6), namely $f \in P_{B}\left(f-\frac{\lambda}{1-\lambda}(f-e)\right)$.

(iii) By Theorem 4.1.2 we know that $T_{A, B}^{\lambda}$ is single-valued at its fixed points if both $A$ and $B$ are super-regular at a distance and assumptions (i)-(iv) of Theorem 4.1.2 hold. The local gap $f-P_{A} f$ is therefore unique. In [98] uniqueness of such gap vectors was an assumption of the convergence analysis. Our results show that we can remove this assumption.

For two sets it is much simpler to describe the geometry. We denote for two closed and convex sets $A$ and $B$ in $\mathcal{E}$ by $E$ (respectively $F$ ) the points in $A$ (respectively $B$ ) that are closest to $B$ (respectively $A$ ). That is,

$$
\begin{aligned}
& E:=\{a \in A \mid \operatorname{dist}(a, B)=\operatorname{dist}(A, B)\}, \\
& F:=\{b \in B \mid \operatorname{dist}(A, b)=\operatorname{dist}(A, B)\} .
\end{aligned}
$$

We denote by

$$
g:=P_{\overline{B-A}}(0)
$$

the displacement vector (or gap), between the sets $A$ and $B$. This construction appeared in many works on 2-set feasibility such as $[11,12,18,97,98]$. 
Example 4.2.5. To illustrate the sets $E$ and $F$ as well as the displacement vector $g$, we consider three small examples. The first consists in two nonintersecting halfspaces and is shown in Fig. 4.4a. The sets $A$ and $B$ are given by

$$
A=(-\infty, 1] \times \mathbb{R} \text { and } B=[2.5, \infty) \times \mathbb{R} .
$$

Here, the sets $E$ and $F$ are both lines describing the boundary of the sets $A$ and $B$ respectively. In particular, $E=\{1\} \times \mathbb{R}$ and $F=\{2.5\} \times \mathbb{R}$. The displacement vector is given by $g=(1.5,0)$. If the sets change to balls, as in Fig. $4.4 \mathrm{~b}$, the nearest points sets $E$ and $F$ change. The sets $A$ and $B$ are described by

$$
A=\left\{x \in \mathbb{R}^{2} \mid x_{1}^{2}+x_{2}^{2} \leq 1\right\} \quad \text { and } B=\left\{x \in \mathbb{R}^{2} \mid\left(x_{1}-4\right)^{2}+x_{2}^{2} \leq 1.5^{2}\right\},
$$

and thus, $E=(1,0)$ and $F=(2.5,0)$. If we shift the set $B$ closer to the set $A$ such that they intersect in exactly one point, the sets $E$ and $F$ collapse to one point, i.e. $E=F=A \cap B$. For a proper treatment of the constructions $E, F$ and $g$ see for instance $[11,12]$.

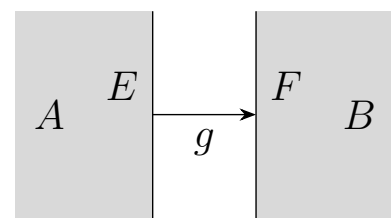

(a) Two nonintersecting sets.

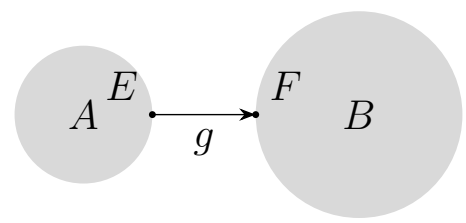

(b) Two nonintersecting balls.

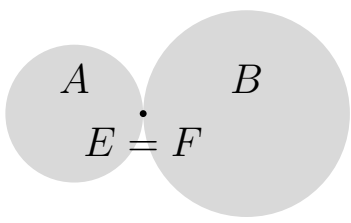

(c) Two intersecting balls.

Figure 4.4: Illustration of the sets $E$ and $F$ and the displacement vector $g$ in the three different settings of Example 4.2.5.

Corollary 4.2.6 (fixed points for closed convex sets [97, Theorem 2.2]). Let $A$ and $B$ be two closed convex subsets of $\mathcal{E}$ and $\lambda \in(0,1)$. Then

$$
\text { Fix } T_{A, B}^{\lambda}=F-\frac{\lambda}{1-\lambda} g \text {. }
$$

In particular, if $A \cap B \neq \emptyset$, then Fix $T_{A, B}^{\lambda}=A \cap B$.

Remark 4.2.7. Although Corollary 4.2.6 was originally proven by Luke in [97], we are able to rediscover it with the presented theory above. The inclusion Fix $T_{A, B}^{\lambda} \subset$ $F-\frac{\lambda}{1-\lambda} g$ for $A$ and $B$ closed and convex is due to convexity of the sets, Theorem 4.2.3 and [11, Fact 1.1]. The other inclusion, $F-\frac{\lambda}{1-\lambda} g \subset$ Fix $T_{A, B}^{\lambda}$, can be verified just by applying $T_{A, B}^{\lambda}$ to a point $x$ in $F-\frac{\lambda}{1-\lambda} g$ and using convexity of the sets.

Corollary 4.2.8 (fixed points of relaxed DR and the corresponding gap). In the setting of Theorem 4.2.3, let $x \in$ Fix $T_{A, B}^{\lambda} \cap U$. Then

$$
\{x\}=P_{B} x-\frac{\lambda}{1-\lambda}\left(P_{B} x-P_{A} P_{B} x\right)
$$


Proof. The result follows directly from the proof of Theorem 4.2.3.

In our statements we require that Fix $T_{A, B}^{\lambda} \neq \emptyset$. Although this assumption is very strong, it is not very restrictive and is satisfied under the assumption of compactness of one of the underlying sets and convexity of both sets.

Proposition 4.2.9 (convexity and compactness imply nonempty fixed point set). Let $\lambda \in(0,1)$. If $A$ and $B$ are convex and closed, and $A$ is bounded, then Fix $T_{A, B}^{\lambda} \neq$ $\emptyset$. Moreover, Fix $T_{A, B}^{\lambda}=\mathcal{M}$, where $\mathcal{M}$ is given by (4.6).

Proof. The proof follows the pattern of the proof in [98, Lemma 2.1], which establishes existence of fixed points for $T_{A, B}^{\lambda}$ by first showing the existence of fixed points of the alternating projections mapping $T:=P_{A} P_{B}$. To see this, note that $T$ is nonexpansive since the projectors $P_{A}$ and $P_{B}$ are nonexpansive, and the composition of nonexpansive mappings is nonexpansive by a similar argument as made in Example 4.1.4(i). Note that $U=\mathcal{E}$. Existence of fixed points of $T$ is then an easy consequence of [35, Theorem 2], which requires that one of the sets, $A$ or $B$, is compact. Let $e \in$ Fix $T$. Then $P_{B} e=f$ and $P_{A} f=e$ and $T_{A, B}^{\lambda}$, by convexity, is single-valued. By Remark 4.2.4(i), we have $f+\frac{\lambda}{1-\lambda}(f-e)=e+\frac{1}{1-\lambda}(f-e)$ and $P_{A}\left(e+\frac{1}{1-\lambda}(f-e)\right)=e$ for all $\lambda \in(0,1)$. Moreover, for all such $\lambda$ we have $f=P_{B}\left(f-\frac{\lambda}{1-\lambda}(f-e)\right)$. Together, for $\bar{x}=f-\frac{\lambda}{1-\lambda}(f-e)$, this yields

$$
\begin{aligned}
T_{A, B}^{\lambda}(\bar{x}) & =\frac{\lambda}{2}\left(R_{A}\left(R_{B}(\bar{x})\right)+\bar{x}\right)+(1-\lambda) P_{B}(\bar{x}) \\
& =\frac{\lambda}{2}\left(R_{A}(2 f-\bar{x})+\bar{x}\right)+(1-\lambda) f \\
& =\frac{\lambda}{2}\left(2 P_{A}\left(e+\frac{1}{1-\lambda}(f-e)\right)-2 f+\bar{x}+\bar{x}\right)+(1-\lambda) f \\
& =\frac{\lambda}{2}(2 e-2 f+2 \bar{x})+(1-\lambda) f \\
& =\lambda\left(f-\frac{\lambda}{1-\lambda}(f-e)\right)+(1-\lambda) f \\
& =f-\lambda \frac{\lambda}{1-\lambda}(f-e)=\bar{x} .
\end{aligned}
$$

Now, applying Theorem 4.2.3 immediately yields Fix $T_{A, B}^{\lambda}=\mathcal{M}$, where $\mathcal{M}$ is given by (4.6). This completes the proof.

The above result on existence relies heavily on convexity. The next example shows a scenario, in which $T_{A, B}^{\lambda}$ has no fixed points.

Example 4.2.10 (empty fixed point set). Let $A$ be the unit circle in $\mathbb{R}^{2}$, i.e.

$$
A:=\left\{x=\left(x_{1}, x_{2}\right) \in \mathbb{R}^{2} \mid x_{1}^{2}+x_{2}^{2}=1\right\},
$$


and $B$ its origin, i.e. $B:=\{(0,0)\}$. In this setting the fixed point set of $T_{A, B}^{\lambda}$ is empty for all $\lambda \in(0,1)$. To prove this we will show by a case distinction that the fixed point set of $T_{A, B}^{\lambda}$ is empty.

First, note that the projectors and reflectors involved in $T_{A, B}^{\lambda}$ are given by

$$
\begin{aligned}
& P_{B}(x)=(0,0) \quad \forall x \in \mathbb{R}^{2} \\
& P_{A}(x)= \begin{cases}\frac{x}{\|x\|} & \forall x \in \mathbb{R}^{2} \backslash(0,0), \\
A & \text { for } x=(0,0) .\end{cases}
\end{aligned}
$$

Now, let $x=(0,0)$. Then

$$
T_{A, B}^{\lambda}(x)=\frac{\lambda}{2}\left(R_{A} R_{B}(x)+x\right)+(1-\lambda) P_{B}(x)=\frac{\lambda}{2}\left(R_{A}(x)\right)=\lambda A .
$$

Thus, $x=(0,0)$ cannot be a fixed point of $T_{A, B}^{\lambda}$. For the other case let $x \neq(0,0)$. Then

$$
T_{A, B}^{\lambda}(x)=\frac{\lambda}{2}\left(R_{A} R_{B}(x)+x\right)+(1-\lambda) P_{B}(x)=\frac{\lambda}{2}\left(R_{A}(-x)+x\right)=\lambda\left(x-\frac{x}{\|x\|}\right) .
$$

If $x$ is a fixed point of $T_{A, B}^{\lambda}$, that is $x=T_{A, B}^{\lambda}(x)$, the following has to hold

$$
x=\lambda\left(x-\frac{x}{\|x\|}\right)
$$

which is equivalent to

$$
\frac{1-\lambda}{\lambda} x=-\frac{x}{\|x\|}
$$

But this is only satisfied when $x=(0,0)$, a contradiction. From which we conclude that $x \notin$ Fix $T_{A, B}^{\lambda}$, and therefore Fix $T_{A, B}^{\lambda}=\emptyset$.

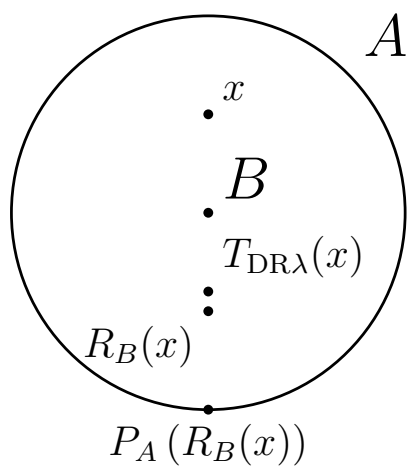

Figure 4.5: Example 4.2.10 for a point $x \in \mathbb{R}^{2}$ and $\lambda=0.8$. 
The following proposition provides a comparison of the fixed points for $T_{A, B}^{\lambda}$ for different values of $\lambda$

Proposition 4.2.11. Let $A$ and $B$ be both closed subsets of $\mathcal{E}$, and $\lambda_{1}, \lambda_{2} \in(0,1)$ such that $\lambda_{1} \leq \lambda_{2}$ and Fix $T_{A, B}^{\lambda_{2}} \neq \emptyset$. Moreover, let $T_{A, B}^{\lambda_{2}}$ be single-valued at its fixed points. Then

$$
P_{B}\left(\text { Fix } T_{A, B}^{\lambda_{2}}\right) \subseteq P_{B}\left(\text { Fix } T_{A, B}^{\lambda_{1}}\right) .
$$

If (4.6) holds for $\lambda_{2}$ with equality instead of just set inclusion, then (4.10) holds with equality.

Proof. Let $x \in$ Fix $T_{\mathrm{DR} \lambda_{2}} \neq \emptyset$. Then, by Corollary 4.2.8, we have the representation

$$
x=P_{B}(x)-\frac{\lambda_{2}}{1-\lambda_{2}}\left(P_{B}(x)-P_{A}\left(P_{B}(x)\right)\right) .
$$

Consider $\tilde{x}:=P_{B}(x)-\frac{\lambda_{1}}{1-\lambda_{1}}\left(P_{B}(x)-P_{A}\left(P_{B}(x)\right)\right)$ and note, as in the statements before, that $P_{B}(x)$ as well as $P_{A}\left(P_{B}(x)\right)$ are single-valued, since $x$ is a fixed point of $T_{\mathrm{DR} \lambda_{2}} \neq \emptyset$. Set $f:=P_{B}(x)$. Then $f \in B$ and $P_{B}(\widetilde{x})=f$. To see this, note that $\frac{\lambda_{2}}{1-\lambda_{2}}\left(P_{A}(f)-f\right) \in N_{B}^{\text {prox }}(f)$. Since $0 \leq \frac{\lambda_{1}}{1-\lambda_{1}} \leq \frac{\lambda_{2}}{1-\lambda_{2}}, \tilde{x}$ is a convex combination of $f$ and $x$, from which we conclude that $P_{B}(\widetilde{x})=f$. Moreover, since $P_{B}(x)=f=P_{B}(\widetilde{x})$, we can conclude that $\widetilde{x} \in$ Fix $T_{\mathrm{DR} \lambda_{1}}$. To see this, evaluate $T_{\mathrm{DR}_{1}}(\widetilde{x})$

$$
\begin{aligned}
T_{\mathrm{DR} \lambda_{1}}(\widetilde{x}) & =\left\{y \mid y \in \lambda_{1}\left(P_{A}\left(R_{B}(\widetilde{x})\right)+\widetilde{x}\right)+\left(1-2 \lambda_{1}\right) P_{B}(\widetilde{x})\right\} \\
& =\left\{y \mid y \in \lambda_{1}\left(P_{A}(2 f-\widetilde{x})+\widetilde{x}\right)+\left(1-2 \lambda_{1}\right) f\right\},
\end{aligned}
$$

since $P_{B}(\widetilde{x})=\{f\} .2 f-\widetilde{x}=2 f-\left(f-\frac{\lambda_{1}}{1-\lambda_{1}}\left(f-P_{A}(f)\right)\right)$, where $P_{A}(f)$ is singlevalued since $x$ is a fixed point of $T_{\mathrm{DR} \lambda_{2}}$. This yields

$$
\begin{aligned}
2 f-\widetilde{x} & =f+\frac{\lambda_{1}}{1-\lambda_{1}}\left(f-P_{A}(f)\right) \\
& =P_{A}(f)+\frac{1}{1-\lambda_{1}}\left(f-P_{A}(f)\right) .
\end{aligned}
$$

Analog to what we have seen before, we can argue that $P_{A}(f) \in P_{A}(2 f-\widetilde{x})$, since $0 \leq \frac{\lambda_{1}}{1-\lambda_{1}} \leq \frac{\lambda_{2}}{1-\lambda_{2}}$ and $P_{A}(f)=P_{A}(2 f-x)=P_{A}\left(f+\frac{\lambda_{2}}{1-\lambda_{2}}\left(f-P_{A}(f)\right)\right)$. This implies that

$$
\begin{aligned}
& \lambda_{1}\left(P_{A}(f)+\widetilde{x}\right)+(1-\left.2 \lambda_{1}\right) f \in T_{\mathrm{DR} \lambda_{1}}(\widetilde{x}) \\
& \Longleftrightarrow \\
& \lambda_{1}\left(P_{A}(f)+f-\frac{\lambda_{1}}{1-\lambda_{1}}\left(f-P_{A}(f)\right)\right)+(1-\left.2 \lambda_{1}\right) f \in T_{\mathrm{DR} \lambda_{1}}(\widetilde{x}) \\
& \Longleftrightarrow \\
& f-\frac{\lambda_{1}}{1-\lambda_{1}}\left(f-P_{A}(f)\right) \in T_{\mathrm{DR} \lambda_{1}}(\widetilde{x}) \\
& \Longleftrightarrow \\
& \widetilde{x} \in T_{\mathrm{DR} \lambda_{1}}(\widetilde{x}),
\end{aligned}
$$

and therefore $\widetilde{x} \in$ Fix $T_{\mathrm{DR} \lambda_{1}}$. In conclusion,

$$
P_{B}\left(\text { Fix } T_{\mathrm{DR} \lambda_{2}}\right) \subseteq P_{B}\left(\text { Fix } T_{\mathrm{DR} \lambda_{1}}\right),
$$

which proves the claim. 


\section{3. $T_{A, B}^{\lambda}$ AT Fix $T_{A, B}^{\lambda}:$ Metric Subregularity}

We now proceed to the main goal of our study, the convergence analysis of the algorithm. Almost all of the key properties of the relaxed Douglas-Rachford fixed point mapping, $T_{A, B}^{\lambda}$, have been established in Section 4.2. In our setting, nonemptiness of the fixed point set and averagedness of the mapping can be identified as the essential properties yielding convergence of the iterative sequence. It was shown in [104], however, that gauge metric subregularity of a fixed point mapping at its fixed points is a necessary condition for quantifiable (by said gauge) rates of convergence of the fixed point iteration. ${ }^{1}$ We have already shown in Theorem 4.1.2 that $T_{A, B}^{\lambda}$ is almost averaged, with any desired violation constant $\epsilon>0$, at its fixed points on certain neighborhoods, when $A$ and $B$ are super-regular at a distance. To achieve local linear convergence, inequality (3.6) in Theorem 3.2.6 must hold. This is where uniformity of almost averagedness with respect to $\epsilon$ is crucial. As long as the mapping $T_{A, B}^{\lambda}-\mathrm{Id}$, or a related mapping (see the discussion below), can be shown to be relatively metrically subregular at 0 on a neighborhood of Fix $T_{A, B}^{\lambda}$ - regardless of the value of the modulus $\kappa$ - suitable neighborhoods can be found in the context of Theorem 4.1.2 where the violation, $\epsilon$, is small enough that (3.6) is satisfied. Hence, local linear convergence is guaranteed. Thus, the main work before us, is to show metric subregularity of the appropriate mapping at points in the product space corresponding to fixed points of $T_{A, B}^{\lambda}$. There are a number of ways to go about this, but all successful strategies we found are based on a characterization of the iterates on neighborhoods of fixed points lifted to a product space where the tools are applied. We were unable to provide a direct approach, involving the $T_{A, B}^{\lambda}$ mapping itself, that guarantees metric subregularity from properties of the regularity of the sets $A$ and $B$ both individually (e.g. relative super-regularity at a distance) or as a collection (e.g. subtransversality discussed below). The characterization of the fixed points in Theorem 4.2.3 allows us to build auxiliary phantom sets that are used in the analysis. To adapt the framework above to the present setting, we build a product space, which represents not only the iterates of $T_{A, B}^{\lambda}$, but also a cyclic projection between the phantom sets. In particular, we define an operator in the product space $\mathcal{E}^{4}$ whose first entry is generated by applying $T_{A, B}^{\lambda}$. The remaining three entries are generated by projecting the prior entry onto the sets $A$ and $B$ as well as phantom versions of these sets shifted by a scaling of the local gap vector between $A$ and $B$ at the reference fixed point.

For the product space formulation, we consider, instead of the two original sets, four sets: the sets $A, B$ and shifted sets $B-\frac{\lambda}{1-\lambda} g$ and $A-\frac{\lambda}{1-\lambda} g$ for some gap vector $g$. Our aim is to show local linear convergence of $T_{A, B}^{\lambda}$ by adapting the approach developed in [105] for cyclic projections, where it was essential that one of the sets involved contains the fixed points of the mapping. The reason for including the set $B-\frac{\lambda}{1-\lambda} g$ in our problem, therefore, lies in the characterization of the fixed point set of the $T_{A, B}^{\lambda}$ mapping. As established in Theorem 4.2 .3 and Corollary 4.2.8, fixed

\footnotetext{
${ }^{1}$ As mentioned in Remark 3.2.4, we focus here on linear gauges.
} 


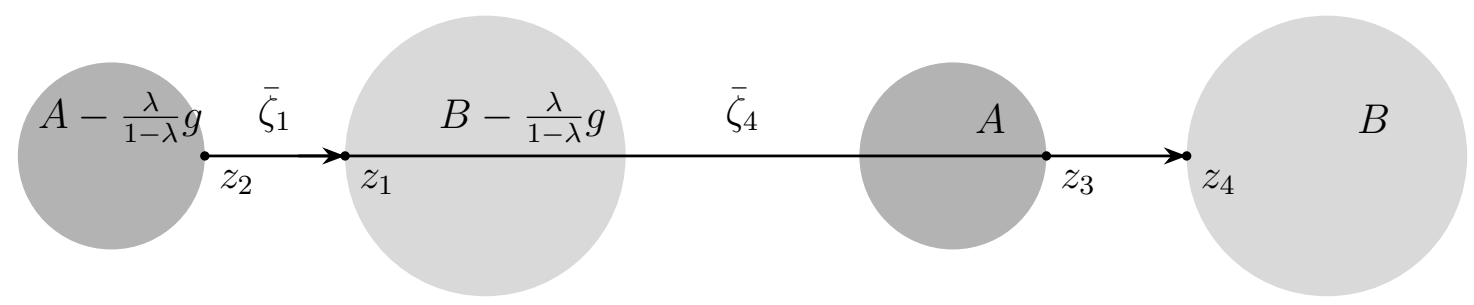

Figure 4.6: Framework for the convergence analysis illustrated in $\mathcal{E}$.

points $\bar{x}$ of $T_{A, B}^{\lambda}$ at which $T_{A, B}^{\lambda}$ is single-valued can be described as

$$
\{\bar{x}\}=P_{B}(\bar{x})-\frac{\lambda}{1-\lambda}\left(P_{B}(\bar{x})-P_{A}\left(P_{B}(\bar{x})\right)\right),
$$

which is an element in $B-\frac{\lambda}{1-\lambda} g$ when $g=P_{B} \bar{x}-P_{A} P_{B} \bar{x}$. Thus, $B-\frac{\lambda}{1-\lambda} g$ contains fixed points of $T_{A, B}^{\lambda}$ that correspond to the gap $g$. In order to apply our results established in Lemma 3.4.2, we have to consider the set $A-\frac{\lambda}{1-\lambda} g$ as well.

We denote by $\Omega_{g}$ the product of the collection of sets $\left\{B-\frac{\lambda}{1-\lambda} g, A-\frac{\lambda}{1-\lambda} g, A, B\right\}$. That is,

$$
\Omega_{g}:=\left(B-\frac{\lambda}{1-\lambda} g\right) \times\left(A-\frac{\lambda}{1-\lambda} g\right) \times A \times B .
$$

Define

$$
\begin{aligned}
W_{0}(g):=\left\{u=\left(u_{1}, u_{2}, u_{3}, u_{4}\right) \in \mathcal{E}^{4} \mid u_{1}\right. & \in P_{B-\frac{\lambda}{1-\lambda} g} u_{2}, \\
u_{2} & \left.\in P_{A-\frac{\lambda}{1-\lambda} g} u_{3}, u_{3} \in P_{A} u_{4}, u_{4} \in P_{B} u_{1}\right\} .
\end{aligned}
$$

This is the set of fixed points of the mapping $P_{\Omega_{g}} \circ \Pi$ in the product space $\mathcal{E}^{4}$ corresponding to a cycle of the cyclic projections operator $P_{B-\frac{\lambda}{1-\lambda} g} P_{A-\frac{\lambda}{1-\lambda} g} P_{A} P_{B}$. By our construction, the set $W_{0}(g)$ could be (and for generic $g$ will be) empty; this would be the case when $g$ does not correspond to a gap vector. The set of difference vectors, $\zeta$, is denoted by $\mathcal{Z}(x, g)$ and defined by

$$
\mathcal{Z}(x, g):=\left\{\zeta:=z-\Pi z \mid z \in W_{0}(g) \subset \mathcal{E}^{4}, z_{1}=x\right\} .
$$

Given an element of the set of difference vector, we define the set

$$
W(\bar{\zeta}):=\left\{u \in \mathcal{E}^{4} \mid u-\Pi u=\bar{\zeta}\right\} .
$$

This set is an affine transformation of the diagonal of the product space and serves as a characterization of the local geometry of the sets in relation to each other at fixed points of $T_{A, B}^{\lambda}$.

These sets, of course, only make sense in the context of local nearest points between the components. In particular, we are interested in points $x \in \mathcal{E}$ associated 
with fixed points of $T_{A, B}^{\lambda}$ and their associated shadow points and gap vectors, respectively $b \in P_{B} x$ and $g \in b-P_{A} b$ (the local gap between $A$ and $B$ ). Note that by Theorem 4.1.2 for fixed points of $T_{A, B}^{\lambda}$ at which $T_{A, B}^{\lambda}$ is single-valued, we have $\{b\}=P_{B} x$ and the gap vector $g$ is unique. When $x$ is a fixed point, the set $\mathcal{Z}(x, g)$ characterizes the distance between the cyclically projected iterates of $T_{A, B}^{\lambda}$ on the individual sets. This enables us to distinguish different fixed points of $T_{A, B}^{\lambda}$ according to their respective difference vectors.

Let the assumptions of Theorem 4.1.2 hold at $\bar{u} \in \mathrm{Fix} T_{A, B}^{\lambda}$ and let $z \in W_{0}(g) \subset \mathcal{E}^{4}$ for $z_{1}=\bar{u}$ and $\{g\}=P_{B}(\bar{u})-P_{A} P_{B}(\bar{u})$. Then Theorem 4.1 .2 yields

(i) $z_{3}-z_{4} \in P_{A}\left(P_{B}\left(z_{1}\right)\right)-P_{B}\left(z_{1}\right)=\{-g\}$

(ii) $z_{4}-z_{1} \in P_{B}\left(z_{1}\right)-\left(P_{B}\left(z_{1}\right)-\frac{\lambda}{1-\lambda} g\right)=\left\{\frac{\lambda}{1-\lambda} g\right\}$,

where (ii) holds by Theorem 4.2 .3 which is applicable since $T_{A, B}^{\lambda}$ is single-valued at $\bar{u}$ by Theorem 4.1.2. Moreover, we get by the assumptions of Theorem 4.1.2 that $P_{A}\left(R_{B}(\bar{u})\right)=P_{A}\left(P_{B}(\bar{u})\right)=z_{3}$. Since $z_{3}+\frac{\lambda}{1-\lambda} g$ lies in a straight line between $R_{B}(\bar{u})=2 P_{B}(\bar{u})-\bar{u}=2 P_{B}(\bar{u})-P_{B}(\bar{u})+\frac{\lambda}{1-\lambda} g=P_{A}\left(P_{B}(\bar{u})\right)+\left(1+\frac{\lambda}{1-\lambda}\right) g$ and $P_{A}\left(R_{B}(\bar{u})\right)$ we also deduce $P_{A}\left(z_{3}+\frac{\lambda}{1-\lambda} g\right)=z_{3}$. Using again Theorem 4.2 .3 yields

(iii) $z_{1}-z_{2} \in P_{B}(\bar{u})-\frac{\lambda}{1-\lambda} g-P_{A-\frac{\lambda}{1-\lambda} g}\left(z_{3}\right)$

$$
\begin{aligned}
& =P_{B}(\bar{u})-\frac{\lambda}{1-\lambda} g-P_{A}\left(z_{3}+\frac{\lambda}{1-\lambda} g\right)+\frac{\lambda}{1-\lambda} g \\
& =P_{B}(\bar{u})-z_{3} \\
& =P_{B}(\bar{u})-P_{A}\left(P_{B}(\bar{u})\right) \\
& =\{g\}
\end{aligned}
$$

(iv) $z_{2}-z_{3}=\left(z_{2}-z_{1}\right)+\left(z_{1}-z_{4}\right)+\left(z_{4}-z_{3}\right)=-\frac{\lambda}{1-\lambda} g$.

Figure 4.6 illustrates the sets and difference vectors above. The individual entries of $z$ relate to the cyclically projected fixed point $x$ on each of the individual sets.

Along with the definitions above, we define the operator

$$
T_{\bar{\zeta}}: \mathcal{E}^{4} \rightrightarrows \mathcal{E}^{4}: u \mapsto\left\{\left(u_{1}^{+}, u_{1}^{+}-\bar{\zeta}_{1}, u_{1}^{+}-\bar{\zeta}_{1}-\bar{\zeta}_{2}, u_{1}^{+}-\bar{\zeta}_{1}-\bar{\zeta}_{2}-\bar{\zeta}_{3}\right) \mid u_{1}^{+} \in T_{A, B}^{\lambda} u_{1}\right\}
$$

for $\bar{\zeta} \in \mathcal{Z}(\bar{x}, g)$, where $\bar{x} \in$ Fix $T_{A, B}^{\lambda}$ and $g=P_{B} \bar{x}-P_{A} P_{B} \bar{x}$. Note that $0=$ $\bar{\zeta}_{1}+\bar{\zeta}_{2}+\bar{\zeta}_{3}+\bar{\zeta}_{4}$, so the expression above can be simplified to

$$
T_{\bar{\zeta}}: \mathcal{E}^{4} \rightrightarrows \mathcal{E}^{4}: u \mapsto\left\{\left(u_{1}^{+}, u_{1}^{+}-\bar{\zeta}_{1}, u_{1}^{+}-\bar{\zeta}_{1}-\bar{\zeta}_{2}, u_{1}^{+}+\bar{\zeta}_{4}\right) \mid u_{1}^{+} \in T_{A, B}^{\lambda} u_{1}\right\} .
$$

The mapping $T_{\bar{\zeta}}$ represents the iterates of $T_{A, B}^{\lambda}$ on the space $\mathcal{E}$ by shifting each iterate by some fixed difference vector $\bar{\zeta}$. We assume, in what follows, that $\bar{\zeta}$ is the difference vector corresponding to the fixed point to which our iteration is converging. Of course, when one does not know the location of the fixed points, it is unlikely that the corresponding difference vector will be known, but this situation is no different than other studies which assume that the problem is consistent, 
and that all fixed points correspond to the zero difference vector. Our aim here is not to determine the difference vector or the fixed point, but rather to provide a quantification of the convergence based on verifiable regularity of the fixed point mapping in neighborhoods of fixed points.

We are now ready to start building our argument. The following lemma establishes a connection between fixed points of $T_{\bar{\zeta}}$ to fixed points of $T_{A, B}^{\lambda}$.

Lemma 4.3.1. Let $\lambda \in(0,1)$ and $A, B \subset \mathcal{E}$ both nonempty and closed. Fix $\bar{x} \in$ Fix $T_{A, B}^{\lambda} \neq \emptyset$ with $T_{A, B}^{\lambda}$ being single-valued at $\bar{x}$ and set $g:=P_{B} \bar{x}-P_{A} P_{B} \bar{x}$. Furthermore, let $\bar{\zeta} \in \mathcal{Z}(\bar{x}, g)$ and define $\Psi_{g}:=\left(P_{\Omega_{g}}\right) \circ \Pi-\Pi$ as well as $\Phi_{\bar{\zeta}}:=T_{\bar{\zeta}}-\mathrm{Id}$. Then the following hold:

(i) $T_{\bar{\zeta}}$ maps $W(\bar{\zeta})$ to itself. Moreover, $u \in$ Fix $T_{\bar{\zeta}}$ if and only if $u \in W(\bar{\zeta})$ with $u_{1} \in \operatorname{Fix} T_{A, B}^{\lambda}$.

(ii)

$$
\Psi_{g}^{-1}(\bar{\zeta}) \cap W(\bar{\zeta}) \cap \mathcal{N} \subseteq \Phi_{\bar{\zeta}}^{-1}(0) \cap W(\bar{\zeta})
$$

where $\mathcal{N}:=\left\{z \in \mathcal{E}^{4} \mid P_{A}\left(z_{4}+\frac{\lambda}{1-\lambda} g\right)=z_{3}\right\}$

(iii) If the distance is with respect to the Euclidean norm, then

$$
\operatorname{dist}\left(0, \Phi_{\bar{\zeta}}(u)\right)=2 \operatorname{dist}\left(u_{1}, T_{A, B}^{\lambda} u_{1}\right)
$$

for $u \in W(\bar{\zeta})$.

Remark 4.3.2. Note that the set $\mathcal{N}$ guarantees equality of the description of the fixed point set in Theorem 4.2.3. In our main result $\mathcal{N}$ will not appear anymore. This is due to the fact that the assumptions of Theorem 4.1.2 assure that the neighborhood we consider will be a subset of $\mathcal{N}$.

Proof of Lemma 4.3.1. (i). The first part of (i) follows immediately by the definition of $T_{\bar{\zeta}}$ and $W(\bar{\zeta})$. Now let $u \in$ Fix $T_{\bar{\zeta}}$,

$$
\begin{array}{ll}
\Longleftrightarrow \quad u_{1} \in \text { Fix } T_{A, B}^{\lambda} \text { and } u_{2}=u_{1}-\bar{\zeta}_{1}, u_{3}=u_{1}-\bar{\zeta}_{1}-\bar{\zeta}_{2}, u_{4}=u_{1}+\bar{\zeta}_{4} \\
\Longleftrightarrow \quad u_{1} \in \text { Fix } T_{A, B}^{\lambda} \text { and } u_{2}=u_{1}-\bar{\zeta}_{1}, u_{3}=u_{2}-\bar{\zeta}_{2}, u_{4}=u_{1}+\bar{\zeta}_{4} \\
\Longleftrightarrow \quad u_{1} \in \text { Fix } T_{A, B}^{\lambda} \text { and } u \in W(\bar{\zeta})
\end{array}
$$

which proves the rest of (i).

(ii). For the second part of the lemma let $z \in \Psi_{g}^{-1}(\bar{\zeta}) \cap W(\bar{\zeta}) \cap \mathcal{N}$. This means nothing more than

$$
\bar{\zeta} \in \Psi_{g}(z) \text { and } z-\Pi z=\bar{\zeta}
$$


which is equivalent to

$$
\bar{\zeta} \in P_{\Omega_{g}} \Pi z-\Pi z \text { and } z-\Pi z=\bar{\zeta} .
$$

This implies

$$
z_{1} \in P_{B-\frac{\lambda}{1-\lambda} g} P_{A-\frac{\lambda}{1-\lambda} g} P_{A} P_{B} z_{1} \text { and } z-\Pi z=\bar{\zeta} .
$$

The mapping $\Phi_{\bar{\zeta}}(z)=T_{\bar{\zeta}} z-z$ has the image $(0,0)$ if $z_{1} \in$ Fix $T_{A, B}^{\lambda} z_{1}$. By $\bar{\zeta}_{4}=z_{4}-z_{1}$ and $\bar{\zeta}_{4} \in P_{B} z_{1}-z_{1}=\frac{\lambda}{1-\lambda} g$ we know that $z_{4} \in P_{B} z_{1}$. This together with the definition of $\mathcal{N}$ yields $P_{A} R_{B} z_{1} \ni P_{A}\left(2 z_{4}-z_{1}\right)=P_{A}\left(z_{4}+\frac{\lambda}{1-\lambda} g\right)=z_{3}$. Inserting this in $T_{A, B}^{\lambda} z_{1}$ yields

$$
\begin{aligned}
T_{A, B}^{\lambda} z_{1} & =\lambda\left(P_{A} R_{B} z_{1}+z_{1}\right)+(1-2 \lambda) P_{B} z_{1} \\
& \ni \lambda\left(z_{3}+z_{1}\right)+(1-2 \lambda) z_{4} \\
& =z_{1}+\lambda\left(z_{3}-z_{4}\right)+(1-\lambda)\left(z_{4}-z_{1}\right) \\
& =z_{1}+\bar{\zeta}_{3}+(1-\lambda) \bar{\zeta}_{4} \\
& =z_{1}
\end{aligned}
$$

since $\bar{\zeta}$ is generated by a fixed point of $T_{A, B}^{\lambda}$. Thus $z_{1} \in$ Fix $T_{A, B}^{\lambda}$, which proves $z \in \Phi_{\bar{\zeta}}^{-1}(0)$ and completes the proof of (ii).

(iii). This part of the proof is a routine calculation:

$$
\begin{aligned}
& \operatorname{dist}\left(0, \Phi_{\bar{\zeta}}(u)\right) \\
= & \operatorname{dist}\left(0, T_{\bar{\zeta}} u-u\right) \\
= & \sqrt{\operatorname{dist}^{2}\left(0, T_{A, B}^{\lambda} u_{1}-u_{1}\right)+\sum_{j=2}^{4} \operatorname{dist}^{2}\left(0, T_{A, B}^{\lambda} u_{1}-\sum_{i=1}^{j-1} \bar{\zeta}_{i}-u_{j}\right)} \\
= & \sqrt{4 \operatorname{dist}^{2}\left(0, T_{A, B}^{\lambda} u_{1}-u_{1}\right)} \\
= & 2 \operatorname{dist}\left(0, T_{A, B}^{\lambda} u_{1}-u_{1}\right) .
\end{aligned}
$$

We present next the main result of this section. In this preliminary result, we show that the mapping $T_{\bar{\zeta}}-$ Id is metrically subregular at its zeros; from this we can conclude that the fixed point iteration generated by the mapping $T_{\bar{\zeta}}$ is locally linear convergent, which implies local linear convergence of $T_{A, B}^{\lambda}$.

Proposition 4.3.3 (metric subregularity of $T_{\bar{\zeta}}$ by subtransversality). Let $\lambda \in$ $(0,1), \bar{x} \in$ Fix $T_{A, B}^{\lambda}$ with $T_{A, B}^{\lambda}$ being single-valued at $\bar{x}$ and set $g:=P_{B} \bar{x}-P_{A} P_{B} \bar{x}$. Furthermore, let $\bar{\zeta} \in \mathcal{Z}(\bar{x}, g)$ and $\bar{u}=\left(\bar{u}_{1}, \bar{u}_{2}, \bar{u}_{3}, \bar{u}_{4}\right) \in W_{0}(g)$ satisfy $\bar{\zeta}=\bar{u}-\Pi \bar{u}$ with $\bar{u}_{1}=\bar{x}$. Let $T_{\bar{\zeta}}$ be defined by (4.15) and define $\Phi_{\bar{\zeta}}:=T_{\bar{\zeta}}-$ Id. Suppose the following hold: 
(i) the collection of sets $\left\{B-\frac{\lambda}{1-\lambda} g, A-\frac{\lambda}{1-\lambda} g, A, B\right\}$ is subtransversal at $\bar{u}$ for $\bar{\zeta}$ relative to $\Lambda \subseteq W(\bar{\zeta})$ with constant $\kappa$ and neighborhood $U$ of $\bar{u}$;

(ii) there exists a positive constant $\sigma$ such that

$$
\operatorname{dist}\left(\bar{\zeta}, \Psi_{g}(u)\right) \leq \sigma \operatorname{dist}\left(0, \Phi_{\bar{\zeta}}(u)\right), \quad \forall u \in \Lambda \cap U \text { with } u_{1} \in B-\frac{\lambda}{1-\lambda} g
$$

Then the mapping $\Phi_{\bar{\zeta}}:=T_{\bar{\zeta}}-$ Id is metrically subregular for 0 on $U$ relative to $\Lambda \cap \mathcal{N}$ with constant $\bar{\kappa}=\kappa \sigma$, where $\mathcal{N}:=\left\{z \in \mathcal{E}^{4} \mid P_{A}\left(z_{4}+\frac{\lambda}{1-\lambda}\right) g=z_{3}\right\}$.

Proof. This is an application of the assumptions and Lemma 4.3.1(ii)

$$
\begin{aligned}
\operatorname{dist}\left(u, \Phi_{\bar{\zeta}}^{-1}(0) \cap \Lambda \cap \mathcal{N}\right) \leq & \operatorname{dist}\left(u, \Psi_{g}^{-1}(0) \cap \Lambda \cap \mathcal{N}\right) \\
\leq & \kappa \operatorname{dist}\left(\bar{\zeta}, \Psi_{g}(u)\right) \\
\leq & \kappa \sigma \operatorname{dist}\left(0, \Phi_{\bar{\zeta}}(u)\right) \\
& \quad\left(\forall u \in U \cap \Lambda \cap \mathcal{N} \text { with } u_{1} \in B-\frac{\lambda}{1-\lambda} g\right)
\end{aligned}
$$

i.e. $\Phi$ is metrically subregular for 0 on $U$ relative to $\Lambda \cap \mathcal{N}$ with constant $\bar{\kappa}$, as claimed.

By Theorem 3.2.6, Proposition 4.3.3 and Theorem 4.1.2, the three ingredients to get convergence are given by the regularity of the sets $A$ and $B$, subtransversality of the collection of sets $\{A, B\}$ and the additional assumption (ii) in Proposition 4.3.3. As seen in [105, Proposition 3.5], this is also true for the alternating projection algorithm. If the intersection $A \cap B$ is nonempty, assuming the stronger property of transversality, super-regularity is enough to show convergence of the DouglasRachford algorithm (see for instance [113, Theorem 6.8] [75, Theorem 3.18]). For alternating projections one only needs transversality at points of intersection and super-regularity of one of the sets [92, Theorem 5.16]. In any case, the additional assumption (ii), is not needed when the assumptions on the fixed points are strong enough. This is also the case for consistent feasibility and the relaxed DouglasRachford method as seen next.

Proposition 4.3.4 (intersecting sets). As before let $\lambda \in(0,1)$. Moreover, assume that the intersection of $A$ and $B$ is nonempty, i.e. $A \cap B \neq \emptyset$. Thus, for every $\bar{x} \in$ $A \cap B \subset$ Fix $T_{A, B}^{\lambda}$ we have $g:=P_{B} \bar{x}-P_{A} P_{B} \bar{x}=0$. Furthermore, let $\bar{\zeta} \in \mathcal{Z}(\bar{x}, g)$. Then (ii) in Proposition 4.3.3 is always satisfied on $\Lambda \subset W(\bar{\zeta})$ with $\sigma=\frac{1}{\sqrt{2} \lambda}$.

Proof. Since $\bar{x} \in A \cap B$ and $g=0$, we get $\bar{\zeta}=(0,0,0,0)$. Moreover, note that for 
every $b \in B$ we gather $T_{A, B}^{\lambda} b-b=\lambda\left(P_{A} b-b\right)$, since

$$
\begin{aligned}
T_{A, B}^{\lambda} b-b & =\frac{\lambda}{2}\left(R_{A} R_{B} b+b\right)+(1-\lambda) P_{B} b-b \\
& =\frac{\lambda}{2}\left(R_{A} b+b\right)+(1-\lambda) b-b \\
& =\lambda P_{A} b-\lambda b \\
& =\lambda\left(P_{A} b-b\right) .
\end{aligned}
$$

Therefore, we deduce for $u \in \Lambda \subset W(\bar{\zeta})=\left\{u \in \mathcal{E}^{4} \mid u_{i}=u_{j}, i, j \in\{1,2,3,4\}\right\}$ with $u_{1} \in B$

$$
\begin{aligned}
T_{0} u-u & =\left(T_{A, B}^{\lambda} u_{1}-u_{1}, T_{A, B}^{\lambda} u_{1}-u_{1}, T_{A, B}^{\lambda} u_{1}-u_{1}, T_{A, B}^{\lambda} u_{1}-u_{1}\right) \\
& =\left(\lambda\left(P_{A} u_{1}-u_{1}\right), \lambda\left(P_{A} u_{1}-u_{1}\right), \lambda\left(P_{A} u_{1}-u_{1}\right), \lambda\left(P_{A} u_{1}-u_{1}\right)\right),
\end{aligned}
$$

and thus

$$
\operatorname{dist}^{2}\left(0, \Phi_{\bar{\zeta}}(u)\right)=\operatorname{dist}^{2}\left(0, T_{0} u-u\right)=4 \operatorname{dist}^{2}\left(0, \lambda\left(P_{A} u_{1}-u_{1}\right)\right) .
$$

On the other hand

$$
\begin{aligned}
\operatorname{dist}^{2}\left(\bar{\zeta}, \Psi_{g}(u)\right) & =\operatorname{dist}^{2}\left((0,0,0,0), \Psi_{0}(u)\right) \\
& =\operatorname{dist}^{2}\left((0,0,0,0), P_{\Omega_{0}} \Pi(u)-\Pi(u)\right) \\
& =2 \operatorname{dist}^{2}\left(0, P_{A} u_{1}-u_{1}\right),
\end{aligned}
$$

since $\Omega_{0}=B \times A \times A \times B$. Combining (4.17) and (4.16) yields (ii) in Proposition 4.3 .3 with $\sigma=\frac{1}{\sqrt{2} \lambda}$.

\subsection{Local Linear Convergence of $T_{A, B}^{\lambda}$}

Lemma 4.4.1 (uniqueness of difference vector for fixed points of $T_{A, B}^{\lambda}$ ). Let $\lambda \in$ $(0,1)$, and let $\bar{x}$ be a point in Fix $T_{A, B}^{\lambda}$, where $A, B \subset \mathcal{E}$ satisfy the assumptions of Theorem 4.1.2 with neighborhoods $U(A, \epsilon, \bar{x})$ and $U(B, \epsilon, \bar{x})$. Then $\{\bar{\zeta}\}=\mathcal{Z}(\bar{x}, g) \subset$ $\mathcal{E}^{4}$ for $\{g\}=P_{B} \bar{x}-P_{A} P_{B} \bar{x}$ is unique and given by

$$
\bar{\zeta}=\left(\bar{\zeta}_{1}, \ldots, \bar{\zeta}_{4}\right)=\left(g,-\frac{\lambda}{1-\lambda} g,-g, \frac{\lambda}{1-\lambda} g\right) .
$$

Proof. By definition (4.13), $\mathcal{Z}(x, g)$ is given by

$$
\mathcal{Z}(x, g):=\left\{\zeta:=z-\Pi z \mid z \in W_{0}(g) \subset \mathcal{E}^{4}, z_{1}=x\right\},
$$

for

$$
W_{0}(g):=\left\{u \in \mathcal{E}^{4} \mid u_{1} \in P_{B-\frac{\lambda}{1-\lambda} g} u_{2}, u_{2} \in P_{A-\frac{\lambda}{1-\lambda} g} u_{3}, u_{3} \in P_{A} u_{4}, u_{4} \in P_{B} u_{1}\right\} .
$$

Thus, the uniqueness of $\bar{\zeta}$ is a direct implication of the uniqueness of $g$ as seen in Remark 4.2.4(iii). 
Next we present the main result. The proof is based on the basic convergence result, Theorem 3.2.6, and the preliminary work done in this chapter.

Theorem 4.4.2 (local linear convergence of $\left.T_{A, B}^{\lambda}\right)$. Let $\lambda \in(0,1)$, and let $\bar{x}$ be a point in Fix $T_{A, B}^{\lambda}$ where $A, B \subset \mathcal{E}$ satisfy the assumptions of Theorem 4.1.2 with neighborhoods $U(A, \epsilon, \bar{x})$ and $U(B, \epsilon, \bar{x})$. Set $\{g\}=P_{B} \bar{x}-P_{A} P_{B} \bar{x}$ and $\{\bar{\zeta}\}=\mathcal{Z}(\bar{x}, g)$ $\left(\bar{\zeta}=\left(\bar{\zeta}_{1}, \ldots, \bar{\zeta}_{4}\right) \in \mathcal{E}^{4}\right)$. Suppose that, at all $x \in$ Fix $T_{A, B}^{\lambda}$ with $g \in P_{B} x-P_{A} P_{B} x$, the sets $A, B \subset \mathcal{E}$ satisfy the assumptions of Theorem 4.1.2 with corresponding neighborhoods $U(A, \epsilon, x)$ and $U(B, \epsilon, x)$. Define the set

$$
S_{0}:=\left\{x \in \operatorname{Fix} T_{A, B}^{\lambda} \mid\{g\}=P_{B} x-P_{A} P_{B} x\right\}
$$

and let

$$
S_{j}:=\left(S_{0}-\sum_{i=1}^{j-1} \bar{\zeta}_{i}\right) \quad(j=1,2,3,4)
$$

Fix some $\epsilon>0$ and define the neighborhood $U_{A}:=\cup_{x \in S_{0}} U(A, \epsilon, x)$ and likewise $U_{B}:=\cup_{x \in S_{0}} U(B, \epsilon, x)$. Then

$$
U:=\left(U_{B}-\frac{\lambda}{1-\lambda} g\right) \times\left(U_{A}-\frac{\lambda}{1-\lambda} g\right) \times U_{A} \times U_{B}
$$

is a neighborhood of $S:=S_{1} \times S_{2} \times S_{3} \times S_{4}$. Suppose that, for $\Lambda \subseteq(W(\bar{\zeta}))$ satisfying $S \subset \Lambda$ with $T_{\bar{\zeta}}: \Lambda \rightrightarrows \Lambda$, the following hold for all $\bar{u}=\left(\bar{u}_{1}, \bar{u}_{2}, \bar{u}_{3}, \bar{u}_{4}\right) \in S$ :

(i) for all $\left(\bar{u}_{3}, \bar{u}_{4}\right) \in S_{3} \times S_{4}$, the collection of sets $\{A, B\}$ is subtransversal at $\left(\bar{u}_{3}, \bar{u}_{4}\right)$ for $\left(\bar{u}_{3}, \bar{u}_{4}\right)-\Pi\left(\bar{u}_{3}, \bar{u}_{4}\right)$ relative to

$$
\Lambda^{\prime}:=\left\{u=\left(u_{1}, u_{2}\right) \in \mathcal{E}^{2} \mid\left(u_{2}-\frac{\lambda}{1-\lambda} g, u_{1}-\frac{\lambda}{1-\lambda} g, u_{1}, u_{2}\right) \in \Lambda\right\}
$$

with constant $\kappa$ on the neighborhood $U_{A} \times U_{B}$;

(ii) for $\Phi_{\bar{\zeta}}:=T_{\bar{\zeta}}-\mathrm{Id}$ and $\Psi_{g}:=P_{\Omega_{g}} \Pi-\Pi$ there exists a positive constant $\sigma$ such that

$$
\operatorname{dist}\left(\bar{\zeta}, \Psi_{g}(u)\right) \leq \sigma \operatorname{dist}\left(0, \Phi_{\bar{\zeta}}(u)\right)
$$

holds whenever $u \in \tilde{\Lambda} \cap U$ with $u_{1} \in B-\frac{\lambda}{1-\lambda} g$ and

$$
\tilde{\Lambda}:=\left\{u \in \Lambda \mid u=\left(x_{2}-\frac{\lambda}{1-\lambda} g, x_{1}-\frac{\lambda}{1-\lambda} g, x_{1}, x_{2}\right) \text { for some } x_{1}, x_{2} \in \mathcal{E}\right\} .
$$

Then there exists an $\epsilon^{\prime} \leq \epsilon$ and a neighborhood $U^{\prime} \subset U\left(U^{\prime}=U_{1}^{\prime} \times U_{2}^{\prime} \times U_{3}^{\prime} \times U_{4}^{\prime} \subset \mathcal{E}^{4}\right)$ of $S$, on which the sequence $\left(u^{k}\right)_{k \in \mathbb{N}}$ generated by $u^{k+1} \in T_{\bar{\zeta}} u^{k}$ seeded by a point $u^{0} \in W(\bar{\zeta}) \cap U^{\prime}$ with $u_{1}^{0} \in U_{1}^{\prime} \cap\left(B-\frac{\lambda}{1-\lambda} g\right)$ satisfies

$$
\operatorname{dist}\left(u^{k+1}, \operatorname{Fix} T_{\bar{\zeta}} \cap S\right) \leq c \operatorname{dist}\left(u^{k}, S\right) \quad(\forall k \in \mathbb{N})
$$


for

$$
c:=\sqrt{1+\epsilon^{\prime}-\frac{1}{\bar{\kappa}^{2}}}<1
$$

where $\bar{\kappa}=\kappa \sigma$ with $\kappa$ and $\sigma$ given by (i) and (ii). Consequently, $\operatorname{dist}\left(u^{k}, \tilde{u}\right) \rightarrow 0$ for some $\tilde{u} \in$ Fix $T_{\bar{\zeta}} \cap S$, and hence

$$
\operatorname{dist}\left(u_{1}^{k}, \tilde{u}_{1}\right) \rightarrow 0
$$

at least $R$-linearly with rate $c<1$. If Fix $T_{A, B}^{\lambda} \cap S_{1}$ is a singleton, then convergence is Q-linear.

Remark 4.4.3 (atlas for the assumptions). At first sight, the assumptions in Theorem 4.4.2 might seem overwhelming. To provide some insight into the statement, we discuss the most important parts of the setting.

1. The assumptions of Theorem 4.1.2 are needed to conclude almost averagedness of $T_{A, B}^{\lambda}$.

2. The requirement that the assumptions of Theorem 4.1.2 hold at all fixed points with the same gap vector is achieved by restricting our analysis to the set $S_{0}$. This also implies that we are considering only fixed points that are isolated relative to $\Lambda$.

3. Although we were not able to prove metric subregularity for a mapping related to $T_{A, B}^{\lambda}$ directly, we can show this property for $T_{\bar{\zeta}}$ on $\mathcal{E}^{4}$. In particular, assumptions (i) and (ii) are used to guarantee metric subregularity from Proposition 4.3.3. Assumption (i) guarantees subtransversality of the collection $\left\{B-\frac{\lambda}{1-\lambda} g, A-\frac{\lambda}{1-\lambda} g, A, B\right\}$ since we have seen in Lemma 3.4.2 that subtransversality is preserved under the addition of some constant vector, here $\frac{\lambda}{1-\lambda} g$.

4. The definitions of $\Lambda^{\prime}$ and $\tilde{\Lambda}$ relate to the construction of the lifted product space version of the problem.

5. The violation $\epsilon$ depends on the violations in Definition 3.3.6 as seen in Theorem 4.1.2. Thus, fixing some violation $\epsilon$ corresponds to certain choices of neighborhoods $U(A, \epsilon, \bar{x})$ and $U(B, \epsilon, \bar{x})$ and violations $\epsilon_{A}$ and $\epsilon_{B}$ of (3.8) for the sets $A$ and $B$ respectively.

Proof of Theorem 4.4.2. First, note that $U$ is a neighborhood of $S$ since $U_{A} \times U_{B}$ is a neighborhood of $S_{3} \times S_{4}$, since for every $(u, \tilde{u}) \in S_{3} \times S_{4}$ there exist $x, \tilde{x} \in S_{0}$ such that $U(A, \epsilon, x) \times U(B, \epsilon, \tilde{x}) \subset U_{A} \times U_{B}$ is a neighborhood of $(u, \tilde{u})$.

The neighborhood $U$ can be replaced by an enlargement of $S$, hence the result follows from Theorem 3.2.6 once it can be shown that the assumptions are satisfied for the mapping $T_{\bar{\zeta}}$ on the product space $\mathcal{E}^{4}$ restricted to $\tilde{\Lambda}$. 
To do so, we note that $T_{A, B}^{\lambda}$ is almost averaged at each $\tilde{y} \in S_{1}$ on $U_{B}$ by Theorem 4.1.2 since the assumptions (i)-(iv) of Theorem 4.1.2 are satisfied. Moreover, following the proof of Theorem 4.1.2 and Proposition 3.1.7 the averaging constant is given by $\alpha:=1 / 2$. Similarly, the violation is given by $\epsilon$ on $U_{B}$. Since $T_{\bar{\zeta}}$ is just $T_{A, B}^{\lambda}$ shifted by $\bar{\zeta}$ on the product space, it follows that $T_{\bar{\zeta}}$ is pointwise almost averaged at $y \in S:=S_{1} \times S_{2} \times S_{3} \times S_{4}$ with the same violation $\epsilon$ and averaging constant $\alpha=1 / 2$ on $U$.

By Lemma 3.4.2 and Remark 3.4.3 assumption (i) therefore implies that for

$$
\bar{u}=\left(\bar{u}_{1}, \bar{u}_{2}, \bar{u}_{3}, \bar{u}_{4}\right) \in S,
$$

the collection of sets

$$
\left\{B-\frac{\lambda}{1-\lambda} g, A-\frac{\lambda}{1-\lambda} g, A, B\right\}
$$

is subtransversal at $\bar{u}$ for $\bar{\zeta}:=\bar{u}-\Pi \bar{u}$ relative to $\tilde{\Lambda}$ with constant $\kappa$ on the neighborhood $U$, hence Theorem 3.2.6(i) is satisfied. Moreover, assumption (ii) of Theorem 4.4.2 and Proposition 4.3.3 with $\mathcal{N}:=\left\{z \in \mathcal{E}^{4} \mid P_{A}\left(z_{4}+\frac{\lambda}{1-\lambda} g\right)=z_{3}\right\} \subset U$ by Theorem 4.1.2(iv) yield assumption Theorem 3.2.6(ii). Altogether, the assumptions of Theorem 3.2.6 are all satisfied for $T_{\bar{\zeta}}$ on $\mathcal{E}^{4}$ restricted to $\tilde{\Lambda}$, and thus we conclude that (3.5) holds.

It remains to show that (3.6) holds, which would imply at least R-linear convergence. To achieve this, choose some $\epsilon^{\prime}>0$ with $\epsilon^{\prime}<\epsilon$ such that (3.6) is satisfied. By Corollary 4.1.3, we can always find neighborhoods $U\left(B, \epsilon^{\prime}, x\right) \subset U(B, \epsilon, x)$ and $U\left(A, \epsilon^{\prime}, x\right) \subset U(A, \epsilon, x)$ for all $x \in S_{0}$ that satisfy the assumptions of Theorem 4.1.2. Following the constructions above, we define $U_{A}^{\prime}:=\cup_{x \in S_{0}} U\left(A, \epsilon^{\prime}, x\right)$ and $U_{B}^{\prime}:=\cup_{x \in S_{0}} U\left(B, \epsilon^{\prime}, x\right)$ and get $U_{A}^{\prime} \subset U_{A}$ as well as $U_{B}^{\prime} \subset U_{B}$. Thus, all the properties that we have shown to be true on $U$ also hold on the subset $U^{\prime}$ defined by

$$
U^{\prime}:=\left(U_{B}^{\prime}-\frac{\lambda}{1-\lambda} g\right) \times\left(U_{A}^{\prime}-\frac{\lambda}{1-\lambda} g\right) \times U_{A}^{\prime} \times U_{B}^{\prime} .
$$

In particular, the constants $\kappa$ and $\sigma$ in (i) and (ii) also suffice for the smaller neighborhoods $U_{A}^{\prime} \times U_{B}^{\prime}$ and $U^{\prime}$. As a consequence, the assumptions of Theorem 3.2.6 are all satisfied and (3.6) holds which implies at least R-linear convergence to $\tilde{u}$. Since $\tilde{u}_{1} \in$ Fix $T_{A, B}^{\lambda} \cap S_{1}$, this completes the proof.

Remark 4.4.4 (a closer look at the convergence statement). The gap vector $g$ and difference vector $\bar{\zeta}$ in Theorem 4.4 .2 rely on the structure of the intersection of the sets $A$ and $B$. The consistent case, that is $A \cap B \neq \emptyset$, leads to a simplification of the problem. Here, the gap is 0. Similarly, the related difference vector is of the form $\bar{\zeta}=\{0,0,0,0\}$. Hence, the assumptions which involve at least one of these vectors can be simplified. When the intersection $A \cap B$ is empty, namely the inconsistent case, the value of $\bar{\zeta}$ is dependent on the choice of $\lambda$. We distinguish three important cases. 
1. $\lambda=\frac{1}{2}$. Here $\frac{\lambda}{1-\lambda}$ reduces to 1 . As a result the phantom sets are shifted by the entire gap $g$ such that $A$ and $B-g$ have a common point. The difference vector is of the form $\bar{\zeta}=\{g,-g,-g, g\}$.

2. $\lambda \rightarrow 1$. Then $\frac{\lambda}{1-\lambda} \rightarrow+\infty$. That is, the phantom sets recede to the horizon in the direction $-g$.

3. $\lambda \rightarrow 0$. In this case $\frac{\lambda}{1-\lambda}$ converges to 0 and the phantom sets coincide in the limit with the original ones. So, $\Omega_{g}=B \times A \times A \times B$. Cyclic projections for these sets $\{B, A, A, B\}$ in the given order is nothing more than alternating projections between the sets $A$ and $B$. At $\lambda=0$, however, Fix $T_{A, B}^{\lambda}=B$, which is clearly larger than the fixed point set for alternating projections.

\subsection{Global Linear Convergence of $T_{A, B}^{\lambda}$}

Although the individual assumptions of Theorem 4.4.2 can be challenging to verify, as we see in Section 4.6, they can reduce to a simpler form if we consider a convex and consistent setting. The reason for this is twofold. First, subtransversality at points in the intersection is nothing more than local linear regularity of the collection of sets [105, Proposition 3.3]. Moreover, it was shown that local linear regularity is equivalent to the global property of linear regularity in the setting of closed convex sets, as seen in [8, Theorem 6.1]. Thus, assuming (i) locally for closed and convex sets, implies that this property holds globally. Second, the technical assumption in (ii) of Theorem 4.4.2 is always satisfied if the feasibility problem is consistent by Proposition 4.3.4. To prove this statement, we first present the auxiliary statements, which are essential to show the global convergence result.

Proposition 4.5.1 (subtransversality at common points [105, Proposition 3.3]). Let $\mathcal{E}^{m}$ be endowed with the 2-norm, that is, $\left\|\left(x_{1}, \ldots, x_{m}\right)\right\|_{2}=\left(\sum_{j=1}^{m}\left\|x_{j}\right\|_{\mathcal{E}}^{2}\right)^{1 / 2}$. A collection $\left\{\Omega_{1}, \Omega_{2}, \ldots, \Omega_{m}\right\}$ of nonempty and closed subsets of $\mathcal{E}$ is subtransversal relative to

$$
\Lambda:=\left\{x=(u, u, \ldots, u) \in \mathcal{E}^{m}|| u \in \mathcal{E}\right\}
$$

at $\bar{x}=(\bar{u}, \bar{u}, \ldots, \bar{u})$ with $\bar{u} \in \cap_{j=1}^{m} \Omega_{j}$ for $\bar{y}=0$ with constant $\kappa$ if there exist a neighborhood $U^{\prime}$ of $\bar{u}$ together with a constant $\kappa^{\prime}$ satisfying $\sqrt{m} \kappa^{\prime} \leq \kappa$ such that

$$
\operatorname{dist}\left(u, \cap_{j=1}^{m} \Omega_{j}\right) \leq \kappa^{\prime} \max _{j=1, \ldots, m} \operatorname{dist}\left(u, \Omega_{i}\right), \quad \forall u \in U^{\prime} .
$$

Conversely, if $\left\{\Omega_{1}, \Omega_{2}, \ldots, \Omega_{m}\right\}$ is subtransversal relative to $\Lambda$ at $\bar{x}$ for $\bar{y}=0$ with constant $\kappa$, then (4.22) is satisfied with any constant $\kappa^{\prime}$ for which $\kappa \leq \kappa^{\prime}$.

The property in (4.22) is called local linear regularity at $\bar{u}$. If the inequality holds for all $u \in \mathcal{E}$, the collection of sets $\{A, B\}$ is said to be linearly regular at $\bar{u}$.

Bakan, Deutsch and Li showed in [8] the equivalence of both properties when the sets are closed and convex. 
Lemma 4.5.2 ([8, Theorem 6.1]). Let the sets $A$ and $B$ be nonempty closed convex sets with $A \cap B \neq \emptyset$. Then the following are equivalent:

(i) There is a $\delta>0$ such that the collection of sets is locally linearly regular at $\bar{x} \in A \cap B$ on $\mathbb{B}_{\delta}(\bar{x})$.

(ii) The collection of sets is linearly regular at $\bar{x} \in A \cap B$.

Having Proposition 4.5.1 and Lemma 4.5.2, we are now ready to state a global convergence result for closed convex sets.

Corollary 4.5.3 (global convergence in the consistent and convex setting). Let $\lambda \in(0,1)$, and let $\bar{x}$ be a point in Fix $T_{A, B}^{\lambda}$. Moreover, let both $A$ and $B$ be closed and convex with $A \cap B \neq \emptyset$ and therefore Fix $T_{A, B}^{\lambda}=A \cap B$. Then $\{g\}=P_{B} \bar{x}-P_{A} P_{B} \bar{x}=$ 0 and $\{\bar{\zeta}\}=\mathcal{Z}(\bar{x}, g)=\{0\}\left(\bar{\zeta}=\left(\bar{\zeta}_{1}, \bar{\zeta}_{2}, \bar{\zeta}_{3}, \bar{\zeta}_{4}\right) \in \mathcal{E}^{4}\right)$. Define the set

$$
S_{0}:=\operatorname{Fix} T_{A, B}^{\lambda}=A \cap B .
$$

Suppose that the following hold for all $\bar{u}=\left(\bar{u}_{1}, \bar{u}_{2}\right) \in S:=S_{0} \times S_{0}$ :

(i) the collection of sets $\{A, B\}$ is subtransversal at $\left(\bar{u}_{1}, \bar{u}_{2}\right)$ for $\left(\bar{u}_{1}, \bar{u}_{2}\right)-\Pi\left(\bar{u}_{1}, \bar{u}_{2}\right)$ relative to $\Lambda^{\prime} \subset\left\{u \in \mathcal{E}^{2} \mid u_{1}=u_{2}\right\}$ with constant $\kappa$ on some neighborhood $U^{\prime} \subset \mathcal{E}^{2}\left(U^{\prime}=U_{A} \times U_{B}\right)$;

Then the sequence $\left(x^{k}\right)_{k \in \mathbb{N}}$ generated by $x^{k+1} \in T_{A, B}^{\lambda} x^{k}$ seeded by a point $x^{0} \in \Lambda^{\prime} \cap U_{B}$ satisfies

$$
\operatorname{dist}\left(x^{k+1}, \operatorname{Fix} T_{A, B}^{\lambda}\right) \leq c \operatorname{dist}\left(x^{k}, \operatorname{Fix} T_{A, B}^{\lambda}\right) \quad(\forall k \in \mathbb{N})
$$

for

$$
c:=\sqrt{1-\frac{\lambda^{2}}{\kappa^{2}}}<1
$$

with $\kappa$ by (i). Consequently, $\operatorname{dist}\left(x^{k}, \tilde{x}\right) \rightarrow 0$ for some $\tilde{x} \in$ Fix $T_{A, B}^{\lambda}$ at least $R$ linearly with rate $c<1$. If Fix $T_{A, B}^{\lambda}$ is a singleton, then convergence is Q-linear.

Remark 4.5.4 (global convergence for convex sets). There are only two changes from Theorem 4.4.2 to Corollary 4.5.3. First, the sets are required to be convex. Thus, convergence in general is guaranteed as stated in Theorem 3.2.1, since convexity of the sets implies averagedness of $T_{A, B}^{\lambda}$ by Proposition 3.3.9, Proposition 3.3.12 and Proposition 3.1.7. Moreover, the local assumption (i) in this case is a global one, i.e. $U^{\prime}=\mathcal{E}^{2}$, by Proposition 4.5.1 and Lemma 4.5.2. The second difference, assumption (ii) in Theorem 4.4.2, is always satisfied by Proposition 4.3.4 since Fix $T_{A, B}^{\lambda}=A \cap B$. 
Proof of Corollary 4.5.3. Since $A$ and $B$ are convex, not only the difference vector is unique, as seen in Lemma 4.4.1, but too is the gap vector $g$ for any fixed point in Fix $T_{A, B}^{\lambda}$. Thus, $S_{0}=$ Fix $T_{A, B}^{\lambda}$. Furthermore, Fix $T_{A, B}^{\lambda}=A \cap B$ by Corollary 4.2.6. With these observations, we get immediately that the sets involved in Theorem 4.4.2 simplify to the following

$$
\begin{aligned}
S & =S_{0} \times S_{0} \times S_{0} \times S_{0}, \\
W(\bar{\zeta}) & =\left\{u \in \mathcal{E}^{4} \mid u-\Pi u=0\right\}=\left\{u \in \mathcal{E}^{4} \mid u_{1}=u_{2}=u_{3}=u_{4}\right\}, \\
U & =U_{B} \times U_{A} \times U_{A} \times U_{B}, \\
\Lambda^{\prime} & \subset\left\{u \in \mathcal{E}^{2} \mid u_{1}=u_{2}\right\},
\end{aligned}
$$

since $\Lambda \subset W(\bar{\zeta})$. Thus, assuming (i) in Corollary 4.5.3 is equivalent to assuming Theorem 4.4.2(i) in the convex and consistent setting. Moreover, since the sets $A$ and $B$ are convex, the projector and reflector across these sets are single-valued (see Proposition 2.2.1 and Corollary 2.2.2). Additionally, the projector is firmly nonexpansive (see Proposition 3.3.9 and Proposition 3.3.12) and thus the reflector is nonexpansive, (see [15, Proposition 4.2]), which implies that $T_{A, B}^{\lambda}$ is averaged with constant $\alpha=(1 / 2)$. The conditions of Theorem 4.1.2 are therefore satisfied with neighborhoods chosen to be $\mathcal{E}$. Also, since the sets $A$ and $B$ are convex, they are super-regular at a distance by Proposition 3.3.9 with $\epsilon=0$. Since every fixed point is an element of the intersection $A \cap B$, we deduce by Proposition 4.3.4 that assumption (ii) of Theorem 4.4.2 holds. The local convergence result follows then from Theorem 4.4.2. What is left to show, is the global convergence property.

By (i) and Proposition 4.5.1, the collection of sets $\{A, B\}$ is locally linearly regular on $U^{\prime}$. Thus, there exists a $\delta>0$ such that $\{A, B\}$ is locally linearly regular on $\mathbb{B}_{\delta}(\bar{x})$. Using Lemma 4.5 .2 we get that $\{A, B\}$ is linearly regular since $A$ and $B$ are convex sets. Altogether, (i) holds with $U^{\prime}=\mathcal{E}^{2}$. That is, the assumption holds globally. Since (ii) of Theorem 4.4.2 holds globally as well by Proposition 4.3.4, the assumptions of the underlying convergence framework in Theorem 3.2.6 hold on $\mathcal{E}$. Therefore, the sequence converges globally, which completes the proof.

Remark 4.5.5 (linking our results to already existing literature). As noted in the introduction, the works $[49,75,94,105,113]$ all analyze the Douglas-Rachford algorithm for consistent nonconvex feasibility. In [105] the framework used here was applied to Douglas-Rachford for structured nonconvex optimization. In [49] the authors showed local R-linear convergence for superregular sets intersecting linear regular. Our analysis of relaxed Douglas-Rachford includes or subsumes that of all previous studies in the context of set feasibility, with the exception of [94], which addresses global convergence guarantees for consistent feasibility. The assumptions of that paper, namely compactness and semi-algebraicity (not to mention nonempty intersection) are different than the notions that we work with. Certainly compactness is a regularity assumption, as is semi-algebraicity or its more general Kurdyka-Łojasiewicz-type regularity, but these notions serve a different purpose. Indeed, even convex sets need not be semi-algebraic or compact. This suggests 
that Kurdyka-Łojasiewicz-type regularity and compactness could be properties in addition to the ones we use in order to arrive at global statements. Nevertheless, as shown in Corollary 4.5.3, in the convex case, the local analysis suffices to infer global convergence properties. A more thorough study of the relationship between the different notions of regularity would be fruitful, but is beyond the scope of our paper.

Our results could be extended to sets with even weaker regularity, namely $\epsilon$ subregular sets instead of super-regular sets at a distance under the additional assumption that suitable neighborhoods exist. But, the present setting is technical enough - increased generality would have only made the details even more difficult to parse. Moreover, the advantage of this specific type of nonconvexity is that we are not only able to present existence results on neighborhoods where we get local convergence, but we are able to construct the neighborhoods explicitly.

\subsection{Elementary Examples}

We demonstrate in this section explicit verification of the assumptions of Theorem Theorem 4.4.2 for a typical class of problems. In particular, we consider the configurations that arise with feasibility problems involving intersecting and nonintersecting spheres in $\mathbb{R}^{2}$. This is of particular interest for the source localization problem and the phase retrieval problem, especially the nonintersecting case. The idealized source localization problem amounts to finding the intersection of spheres that are determined by distance measurements to receivers whose locations are known. When the distance measurements are noisy, or the given locations of the receivers are inaccurate, the intersection over all spheres will be empty almost always. For phase retrieval, the measurements are pointwise amplitude measurements in the Fourier domain of an unknown object. In other words, the constraint sets are two-dimensional spheres in the image of a linear transformation. Since both the measurements and the object are assumed to have compact support, the phase retrieval problem in diffraction imaging is fundamentally inconsistent. In our development below, we carry out the explicit calculations to verify the assumptions of Theorem Theorem 4.4.2 for circles (spheres in $\mathbb{R}^{2}$ ) which was shown in [103] to be the fundamental geometry for phase retrieval and source location problems. Affine subspaces are included as spheres centered at infinity.

There are 5 distinct cases to consider: 1. intersecting circles, 2. nonintersecting separable circles, 3. nonintersecting, nonseparable, nonconcentric circles, 4. nonintersecting concentric circles, and 5. tangential circles. We show that the verification can be carried out "by hand" in the first example. For the sake of brevity, the verification is carried out in the remaining examples with the help of symbolic computation. We were unable to prove or disprove that the required conditions hold in Example 4. In Example 5 we determine that the assumptions are not satisfied, and therefore the algorithm cannot converge linearly. 
The symbolic worksheets where our calculations were carried out are available at http://vaopt.math.uni-goettingen.de/en/publications.php.

For this entire section let $R$ be a positive real-valued number and $\lambda \in(0,1)$ if not specified. To verify subtransversality and the technical condition (ii) in Theorem 4.4.2 we often did not calculate the constants explicitly but bounded them from below. That is,

$$
\begin{aligned}
& \kappa> \frac{\operatorname{dist}\left(u, \Psi_{g}^{-1}(\bar{\zeta}) \cap W(\bar{\zeta})\right)}{\operatorname{dist}\left(\bar{\zeta}, \Psi_{g}(u)\right)}, \\
& \sigma>\frac{\operatorname{dist}\left(\bar{\zeta}, \Psi_{g}(u)\right)}{\operatorname{dist}\left(0, \Phi_{\bar{\zeta}}(u)\right)}
\end{aligned}
$$

where $\kappa$ was the constant of subtransversality and $\sigma$ describes the technical condition. In this section we deal with neighborhoods of fixed points. As a consequence, the constants computed bound the rate of linear convergence from below in such cases. Note that we can always find a neighborhood such that the convergence is linear for examples consisting of two circles by Theorem 4.1.2 and Example 4.1.4.

Example 4.6.1 (two intersecting circles). The first example consists of two circles intersecting at exactly two points. Without loss of generality we can restrict the analysis to the following setting

$$
\begin{aligned}
& A:=\left\{x=\left(x_{1}, x_{2}\right) \in \mathbb{R}^{2} \mid x_{1}^{2}+x_{2}^{2}=1\right\} \\
& B:=\left\{x=\left(x_{1}, x_{2}\right) \in \mathbb{R}^{2} \mid x_{1}^{2}+\left(x_{2}-a\right)^{2}=R^{2}\right\},
\end{aligned}
$$

where $a \in \mathbb{R} \backslash\{0\}$ and $R \in\left(\min _{y \in A} \operatorname{dist}((0, a), y), \max _{y \in A} \operatorname{dist}((0, a), y)\right)$. Note

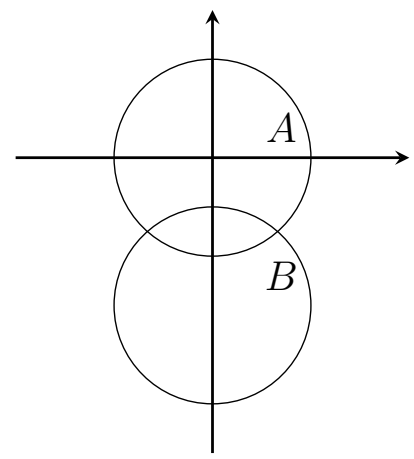

Figure 4.7: Illustration of the sets in Example 4.6.1 for $a=-1.5$ and $R=1$.

that the endpoints of the interval for $R$ correspond to the setting of two touching circles, see Example 4.6.5.

First, we consider the points in the intersection $A \cap B$, namely

$$
\left( \pm \sqrt{1-\left(\frac{1-R^{2}+a^{2}}{2 a}\right)^{2}}, \frac{1-R^{2}+a^{2}}{2 a}\right) .
$$


Due to the symmetry of the problem we restrict the analysis to the point

$$
\bar{u}:=\left(+\sqrt{1-\left(\frac{1-R^{2}+a^{2}}{2 a}\right)^{2}}, \frac{1-R^{2}+a^{2}}{2 a}\right) .
$$

The following statements regarding the assumptions made in Theorem 4.4.2 are easily verified either by hand or with the help of symbolic computation.

(i) $S_{0}:=\left\{\left(+\sqrt{1-\left(\frac{1-R^{2}+a^{2}}{2 a}\right)^{2}}, \frac{1-R^{2}+a^{2}}{2 a}\right)\right\} \in$ Fix $T_{A, B}^{\lambda}$

(ii) In $\mathbb{R}_{+} \times \mathbb{R}$ there is a unique fixed point. $\bar{x}=(\bar{u}, \bar{u}, \bar{u}, \bar{u})$.

(iii) The difference vector is unique and given by $\bar{\zeta}=((0,0),(0,0),(0,0),(0,0))$, since $\bar{u} \in A \cap B$.

(iv) The sets $A$ and $B$ satisfy the assumptions of Theorem 4.1 .2 at $\bar{u}$ with neighborhoods $U_{1}$ and $U_{2}$ being open balls around $\bar{u}$, that is $\mathbb{B}_{\delta}(\bar{u})$, for $\delta \in(0,1)$. This can be shown similar to Example 4.1.4.

(v) This example considers a setting with nonempty intersection. As seen in Proposition 4.5.1 showed by Luke, Thao and Tam, one can equivalently prove linear regularity to get subtransversality in such instances.

Our aim is to use Proposition 4.5.1, proving that Example 4.6.1 satisfies (4.22).

For this we assume that $u \in U_{1}=U_{2}$ is an element of $A$. Additionally, we take the value of $u_{1}$ larger than 0 . We can do this since the statements in Theorem 4.4.2 are all with respect to the set $\Lambda$ which is a subset of $W(\bar{\zeta})$. Thus, the restriction to one of the sets is no contradiction. $u_{1}>0$ ensures that we always project onto the chosen point in the intersection, $\bar{u}$. Then, we have to show

$$
\operatorname{dist}(u, A \cap B) \leq \kappa^{\prime} \max \{\operatorname{dist}(u, A), \operatorname{dist}(u, B)\} \text {, }
$$

which simplifies to

$$
\operatorname{dist}(u, A \cap B) \leq \kappa^{\prime} \operatorname{dist}(u, B) \text {, }
$$

which we reformulate in the following to

$$
\left\|u-P_{A \cap B} u\right\| \leq \kappa^{\prime}\left\|u-P_{B} u\right\| .
$$

Note that (4.23) is equivalent to (4.22) since $u \in A$ and thus implies linear regularity. 
Next, we show (4.23).

$$
\begin{aligned}
\left\|u-P_{A \cap B} u\right\| & \leq\left\|u-P_{B} u\right\|+\left\|P_{B} u-P_{A \cap B} u\right\| \\
& \leq\left\|u-P_{B} u\right\|\left(1+\frac{\left\|P_{B} u-P_{A \cap B} u\right\|}{\left\|u-P_{B} u\right\|}\right)
\end{aligned}
$$

Thus, it remains to show that $1+\frac{\left\|P_{B} u-P_{A \cap B} u\right\|}{\left\|u-P_{B} u\right\|}$ is bounded above by a nonnegative constant.

By construction we get for the individual projections

$$
\begin{aligned}
P_{A \cap B} u & =\left(+\sqrt{1-\left(\frac{1-R^{2}+a^{2}}{2 a}\right)^{2}}, \frac{1-R^{2}+a^{2}}{2 a}\right), \\
P_{B} u & =(0, a)+\frac{u-(0, a)}{\|u-(0, a)\|} R .
\end{aligned}
$$

Inserting this in the above expression yields

$$
\begin{aligned}
& 1+\frac{\left\|P_{B} u-P_{A \cap B} u\right\|}{\left\|u-P_{B} u\right\|} \\
&= 1+\frac{\left\|(0, a)+\frac{u-(0, a)}{\|u-(0, a)\|} R-\left(\sqrt{1-\left(\frac{1-R^{2}+a^{2}}{2 a}\right)^{2}}, \frac{1-R^{2}+a^{2}}{2 a}\right)\right\|}{\left\|u-(0, a)+\frac{u-(0, a)}{\|u-(0, a)\|} R\right\|} \\
& \leq 2+\frac{\left\|u-\left(\sqrt{1-\left(\frac{1-R^{2}+a^{2}}{2 a}\right)^{2}}, \frac{1-R^{2}+a^{2}}{2 a}\right)\right\|}{\left\|u-(0, a)+\frac{u-(0, a)}{\|u-(0, a)\|} R\right\|} \\
&<2+\frac{1}{\left\|u-(0, a)+\frac{u-(0, a)}{\|u-(0, a)\|} R\right\|},
\end{aligned}
$$

since $u \in A$ and thus $\left\|u-\left(\sqrt{1-\left(\frac{1-R^{2}+a^{2}}{2 a}\right)^{2}}, \frac{1-R^{2}+a^{2}}{2 a}\right)\right\|<1$. The denominator

$$
\left\|u-(0, a)+\frac{u-(0, a)}{\|u-(0, a)\|} R\right\|
$$

can be bounded as follows.

Since $u \in A$, we get

$$
\min _{y \in A} \operatorname{dist}((0, a), y) \leq\|u-(0, a)\| \leq \max _{y \in A} \operatorname{dist}((0, a), y) .
$$

And equivalently

$$
\frac{1}{\min _{y \in A} \operatorname{dist}((0, a), y)} \geq \frac{1}{\|u-(0, a)\|} \geq \frac{1}{\max _{y \in A} \operatorname{dist}((0, a), y)} .
$$


Thus,

$$
1-\frac{R}{\|u-(0, a)\|} \geq 1-\frac{R}{\max _{y \in A} \operatorname{dist}((0, a), y)}
$$

which implies

$$
\rho\left(1-\frac{R}{\|u-(0, a)\|}\right) \geq \min _{y \in A} \operatorname{dist}((0, a), y)\left(1-\frac{R}{\max _{y \in A} \operatorname{dist}((0, a), y)}\right),
$$

for $\rho:=\|u-(0, a)\|$. Hence

$$
\frac{1}{\rho\left(1-\frac{R}{\|u-(0, a)\|}\right)} \leq \frac{1}{\min _{y \in A} \operatorname{dist}((0, a), y)\left(1-\frac{R}{\max _{y \in A} \operatorname{dist}((0, a), y)}\right)}=: \kappa^{\prime} .
$$

Since

$$
R \in\left(\min _{y \in A} \operatorname{dist}((0, a), y), \max _{y \in A} \operatorname{dist}((0, a), y)\right),
$$

$\kappa^{\prime}$ is bounded above.

Altogether, $A \cap B$ is locally linear regular at $\left(+\sqrt{1-\left(\frac{1-R^{2}+a^{2}}{2 a}\right)^{2}}, \frac{1-R^{2}+a^{2}}{2 a}\right)$.

By Proposition 4.5.1 we deduce subtransversality with constant $\kappa:=\kappa^{\prime} \sqrt{2}$.

(vi) The technical condition (ii) in Theorem 4.4.2 is satisfied with

$$
\sigma^{2}=\frac{1}{2 \lambda^{2}}
$$

by Proposition 4.3.4.

Thus, the assumptions of Theorem 4.4.2 are satisfied and the relaxed DouglasRachford algorithm converges locally linear to $\bar{u}$ with rate $1>c>\sqrt{1-\frac{\lambda^{2}}{\kappa^{\prime 2}}}$ as long as the starting point is close enough to $\bar{u}$.

Similarly, this argument can be repeated for $\left(-\sqrt{1-\left(\frac{1-R^{2}+a^{2}}{2 a}\right)^{2}}, \frac{1-R^{2}+a^{2}}{2 a}\right)$, which shows that, in this situation, both subtransversality and the technical condition at the two points in the intersection are satisfied.

Note that the point $(0,-1)$ does not lead to a fixed point of the relaxed DouglasRachford algorithm. Whereas, for the Alternating Projection method, defined by the operator $P_{A} P_{B},(0,-1)$ is always a fixed point. In particular, for any $\lambda \in(0,1)$ the fixed point set of $T_{A, B}^{\lambda}$ does not contain any point of the form $(0, y)$ for $y \in \mathbb{R}$.

Example 4.6.2 (nonintersecting separable circles). This example consists of two circles in $\mathbb{R}^{2}$ that are shifted by some vector in $\mathbb{R}^{2}$ such that they do not intersect in any point. Let $R>0$ and define

$$
\begin{aligned}
A & :=\left\{x=\left(x_{1}, x_{2}\right) \in \mathbb{R}^{2} \mid x_{1}^{2}+x_{2}^{2}=1\right\} \\
\text { and } \quad B & :=\left\{x=\left(x_{1}, x_{2}\right) \in \mathbb{R}^{2} \mid\left(x_{1}-(2+R)\right)^{2}+x_{2}^{2}=R^{2}\right\} .
\end{aligned}
$$




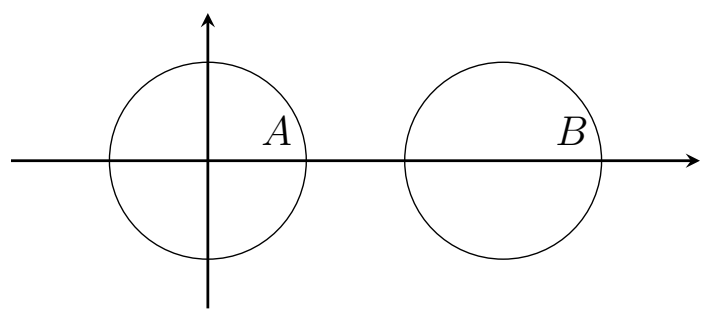

Figure 4.8: Illustration of the sets in Example 4.6.2 for $R=1$.

The only fixed point of $T_{A, B}^{\lambda}$ on $A$ and $B$ is given by

$$
\bar{u}=(2,0)-\frac{\lambda}{1-\lambda}(1,0)
$$

for $\lambda \in(0,1)$. The following statements regarding the assumptions made in Theorem 4.4 .2 are easily verified either by hand or with the help of symbolic computation.

(i) $S_{0}:=\operatorname{Fix} T_{A, B}^{\lambda}=\{\bar{u}\}$.

(ii) The difference vector is unique as well and given by

$$
\bar{\zeta}=\left((1,0),-\frac{\lambda}{1-\lambda}(1,0),(-1,0), \frac{\lambda}{1-\lambda}(1,0)\right) .
$$

(iii) As noted in Example 4.1.4(ii) the assumptions of Theorem 4.1.2 are satisfied for neighborhoods chosen as tubes.

(iv) The modulus of subtransversality $\kappa$ bounded below as follows

$$
\kappa^{2}>\frac{8\left(R^{2}+2 R+1\right)}{R^{2}+2 R+5} .
$$

(v) The technical assumption (ii) in Theorem 4.4.2 is bounded below as follows

$$
\sigma^{2}>\gamma_{1} / 16 \gamma_{2}
$$

where the constants $\gamma_{1}$ and $\gamma_{2}$ were calculated with SageMath and are given by

$$
\begin{aligned}
\gamma_{1}=4\left(R^{4}-\right. & \left.2 R^{2}+1\right) \lambda^{6}-8\left(3 R^{4}+R^{3}-5 R^{2}-R+2\right) \lambda^{5} \\
& +\left(53 R^{4}+40 R^{3}-54 R^{2}-32 R+25\right) \lambda^{4}+4 R^{4} \\
& -2\left(27 R^{4}+33 R^{3}+7 R^{2}-21 R+18\right) \lambda^{3}+8 R^{3} \\
+ & \left(29 R^{4}+42 R^{3}+69 R^{2}-40 R+36\right) \lambda^{2}+20 R^{2} \\
& -4\left(3 R^{4}+4 R^{3}+11 R^{2}-10 R\right) \lambda
\end{aligned}
$$




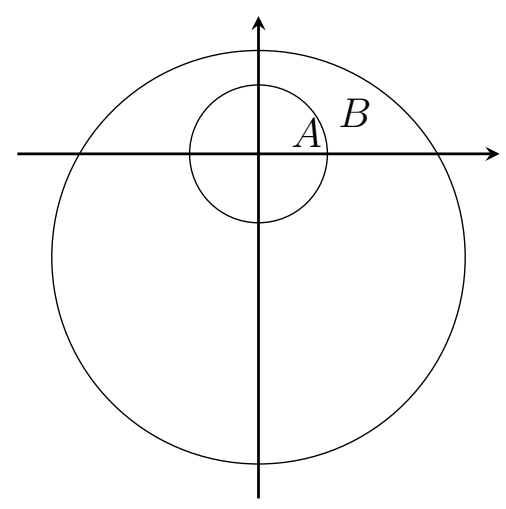

Figure 4.9: Illustration of the sets in Example 4.6.3 for $R=1$.

$$
\begin{aligned}
\gamma_{2}=\left(R^{4}+6 R^{3}+\right. & \left.13 R^{2}+12 R+4\right) \\
-2\left(R^{4}+6 R^{3}+\right. & \left.13 R^{2}+12 R+4\right) \lambda^{3} \\
& +\left(R^{4}+6 R^{3}+13 R^{2}+12 R+4\right) \lambda^{2} .
\end{aligned}
$$

Thus, the assumptions of Theorem 4.4 .2 are satisfied and the relaxed DouglasRachford algorithm converges locally linear to $\bar{u}$ with rate $1>c>\sqrt{1-\frac{1}{(\kappa \sigma)^{2}}}$ as long as the starting point is close enough to $\bar{u}$.

Example 4.6.3 (nonintersecting, nonseparable and nonconcentric circles). This example consists of two circles having not the same center with one surround the other. Let $R>0$ and set

$$
\begin{aligned}
& A:=\left\{x=\left(x_{1}, x_{2}\right) \in \mathbb{R}^{2} \mid x_{1}^{2}+x_{2}^{2}=1\right\} \\
& B:=\left\{x \in \mathbb{R}^{2} \mid x_{1}^{2}+\left(x_{2}-\left(-\frac{1}{2}-R\right)\right)=(2+R)^{2}\right\} .
\end{aligned}
$$

Our analysis considers the fixed point

$$
\bar{u}=\left(0, \frac{3}{2}\right)-\frac{\lambda}{1-\lambda}\left(0, \frac{1}{2}\right)
$$

of $T_{A, B}^{\lambda}$ on $A$ and $B$ for $\lambda<2 / 3$. The following statements regarding the assumptions made in Theorem 4.4 .2 are easily verified either by hand or with the help of symbolic computation.

(i) $S_{0}:=\{\bar{u}\} \in \operatorname{Fix} T_{A, B}^{\lambda}$.

(ii) The difference vector is unique as well and given by

$$
\bar{\zeta}=\left(\left(0, \frac{1}{2}\right),-\frac{\lambda}{1-\lambda}\left(0, \frac{1}{2}\right),-\left(0, \frac{1}{2}\right),-\frac{\lambda}{1-\lambda}\left(0, \frac{1}{2}\right)\right) .
$$

(iii) Similar to the analysis made in Example 4.1.4(ii) the assumptions of Theorem 4.1.2 are satisfied for neighborhoods chosen as tubes. 
(iv) The modulus of subtransversality $\kappa$ is bounded below as follows

$$
\kappa^{2}>\frac{9\left(4 R^{2}+12 R+9\right)}{2 R^{2}+6 R+9}
$$

(v) The technical assumption (ii) of Theorem 4.4.2 is bounded below by

$$
\sigma^{2}>\frac{\gamma_{1}}{9 \gamma_{2}}
$$

where the constants $\gamma_{1}$ and $\gamma_{2}$ were calculated with SageMath and are given by

$$
\begin{aligned}
\gamma_{1}= & 4\left(40 R^{4}+320 R^{3}+958 R^{2}+1272 R+639\right) \lambda^{6} \\
& -4\left(216 R^{4}+1688 R^{3}+5000 R^{2}+6675 R+3438\right) \lambda^{5} \\
& +\left(1864 R^{4}+14208 R^{3}+41826 R^{2}+56844 R+30591\right) \lambda^{4}+144 R^{4} \\
- & \left(2112 R^{4}+15736 R^{3}+46466 R^{2}+65427 R+37485\right) \lambda^{3}+1008 R^{3} \\
+ & \left(1432 R^{4}+10504 R^{3}+31387 R^{2}+46164 R+28071\right) \lambda^{2}+2952 R^{2} \\
& -24\left(26 R^{4}+188 R^{3}+563 R^{2}+843 R+522\right) \lambda+4320 R+2592
\end{aligned}
$$

and

$$
\begin{aligned}
\gamma_{2}=\left(16 R^{4}+64\right. & \left.R^{3}+88 R^{2}+48 R+9\right) \lambda^{6} \\
- & 6\left(16 R^{4}+64 R^{3}+88 R^{2}+48 R+9\right) \lambda^{5} \\
+ & 13\left(16 R^{4}+64 R^{3}+88 R^{2}+48 R+9\right) \lambda^{4} \\
- & 12\left(16 R^{4}+64 R^{3}+88 R^{2}+48 R+9\right) \lambda^{3} \\
& +4\left(16 R^{4}+64 R^{3}+88 R^{2}+48 R+9\right) \lambda^{2} .
\end{aligned}
$$

Thus, the assumptions of Theorem 4.4.2 are satisfied and the relaxed DouglasRachford algorithm converges locally linear to $\bar{u}$ with rate $1>c>\sqrt{1-\frac{1}{(\kappa \sigma)^{2}}}$, as long as the starting point is close enough to $\bar{u}$.

Example 4.6.4 (nonintersecting, nonseparable concentric circles). In comparison to Example 4.6.3, the only thing we change is that we do not allow the circles to have different centers. Let $R>0$ and define

$$
\begin{aligned}
& A:=\left\{x=\left(x_{1}, x_{2}\right) \in \mathbb{R}^{2} \mid x_{1}^{2}+x_{2}^{2}=1\right\} \\
& B:=\left\{x=\left(x_{1}, x_{2}\right) \in \mathbb{R}^{2} \mid x_{1}^{2}+x_{2}^{2}=R^{2}\right\},
\end{aligned}
$$

where we restrict $R$ to be strictly greater that 1 , i.e. $R>1^{2}$. Our analysis focuses

\footnotetext{
${ }^{2}$ For $R<1$ we can change the roles of $A$ and $B$, which results in the situation presented here.
} 


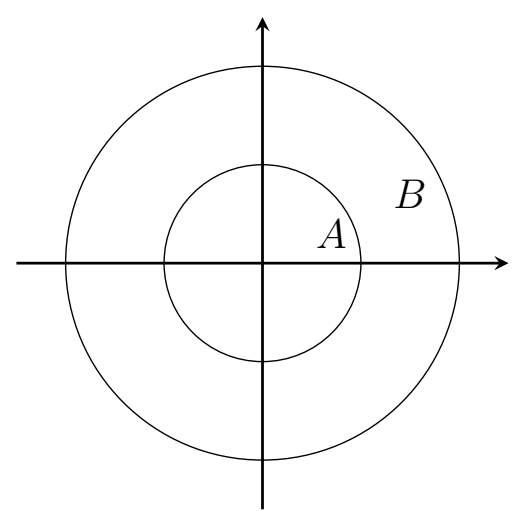

Figure 4.10: Illustration of the sets in Example 4.6.4 for $R=2$.

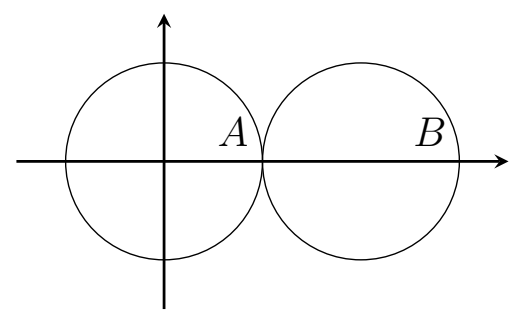

Figure 4.11: Illustration of the sets in Example 4.6.5 for $R=1$.

on the fixed point

$$
\bar{u}=(0, R)-\frac{\lambda}{1-\lambda}(0, R-1)
$$

of $T_{A, B}^{\lambda}$ on $A$ and $B$. Note that it is enough to consider $\bar{u}$ to get the analysis for any other fixed point due to the symmetry of the problem instance.

Unfortunately, we were unable to verify the technical assumption (ii) in Theorem 4.4.2.

Nevertheless, this example is subtransversal. The modulus of subtransversality $\kappa$ is bounded as follows

$$
\kappa^{2}>\frac{2 R^{2}}{R^{4}-2 R^{3}+2 R^{2}-2 R+1} .
$$

Example 4.6.5 (tangential circles). Example 4.6.5 consists of 2 circles touching at a single point. Let $R>0$ and define

$$
\begin{aligned}
& A:=\left\{x=\left(x_{1}, x_{2}\right) \in \mathbb{R}^{2} \mid x_{1}^{2}+x_{2}^{2}=1\right\} \\
& B:=\left\{x=\left(x_{1}, x_{2}\right) \in \mathbb{R}^{2} \mid\left(x_{1}-(R+1)\right)^{2}+x_{2}^{2}=R^{2}\right\} .
\end{aligned}
$$

Our convergence analysis focuses on the only point in the intersection of those two sets, namely

$$
\bar{u}=(1,0) .
$$


The following statements regarding the assumptions made in Theorem 4.4.2 are easily verified either by hand or with the help of symbolic computation.

(i) $S_{0}:=\{\bar{u}\} \in \operatorname{Fix} T_{A, B}^{\lambda}$.

(ii) The difference vector is unique as well and given by

$$
\bar{\zeta}=((0,0),(0,0),(0,0),(0,0)) \text {. }
$$

(iii) The sets $A$ and $B$ satisfy the assumptions of Theorem 4.1.2 at $\bar{u}$ with neighborhoods $U_{1}$ and $U_{2}$ being open balls around $\bar{u}$, that is $\mathbb{B}_{\delta}(\bar{u})$, for $\delta \in(0,1)$.

(iv) The technical condition (ii) in Theorem 4.4.2 is satisfied with

$$
\sigma^{2}=\frac{1}{2 \lambda^{2}}
$$

by Proposition 4.3.4.

(v) However, this example is not subtransversal when examining it in $\mathbb{R}^{2}$. Since $\bar{u}$ is a point in the intersection of $A$ and $B$, we equivalently check linear regularity of the two sets (see Proposition 4.5.1). This yields a constant of linear regularity of

$$
\frac{2 R}{(R+1) b}
$$

where we parametrized a point in the neighborhood of $\bar{u}$ intersected with $A$ as

$$
\left(\sqrt{1-b^{2}}, b\right), b \in[1,-1] \text {. }
$$

Letting $b$ going to 0 (respectively a point close to $\bar{u}$ ) yields a value of $\infty$ in (4.24). This implies that Example 4.6 .5 can not be linearly regular at the point $\bar{u}=(1,0)$ and thus is not subtransversal.

The assumptions of Theorem 4.4.2 therefore are not satisfied. In light of the necessity of metric subregularity for linear convergence [104, Theorem 2], we conclude that $T_{A, B}^{\lambda}$ cannot be linearly convergent in this case (though it might be sublinearly convergent).

Remark 4.6.6. As shown in the examples above the constants involved for both subtransversality and the technical condition (ii) in Theorem 4.4 .2 can be cumbersome although the actual problem might look relatively easy. We also see in Example 4.6.2-Example 4.6.4 that the presence of subtransversality in the inconsistent case can come as a surprise. Our inability to show the technical condition (ii) in Example 4.6.4 indicates that this condition characterizes the regularity or nondegeneracy of the underlying model space for the algorithm. Further investigation of this property is needed. 


\subsection{Special Case: Subspaces}

In the previous sections, we considered super-regular sets at a distance and specialized to convex sets in Corollary 4.5.3. The statements so far about linear convergence depend on the regularity of the individual sets and the whole collection of sets. The aim of this section is to present a class of sets that always satisfies all of the assumptions we require to conclude convergence. In fact, we analyze the relaxed Douglas-Rachford method for two intersecting linear subspaces. As is the case for results on the method of alternating projections for subspaces [2,51-54] and the Douglas-Rachford algorithm for subspaces [19], the notion of the Friedrichs angle is of great importance. In the following, we consider (linear) subspaces. Moreover, note that in finite dimensions subspaces are always closed (see for example [51, Lemma 9.36]). Whenever this specification of subspaces is omitted, we mean closed subspaces. To start, we note the following two facts.

Lemma 4.7.1 (subspaces are super-regular at a distance). Let $\Omega \subset \mathcal{E}$ be a subspace. Then $\Omega$ is super-regular at a distance relative to $\Lambda=\mathcal{E}$ at any $\bar{x} \in \Omega$ and convex.

Proof. Since subspaces are convex by definition, this follows immediately by Proposition 3.3.9.

Lemma 4.7.2 (subspaces always intersect linear regularly). Let $A$ and $B$ be two subspaces in $\mathcal{E}$. Then $\{A, B\}$ is linear regular at every $\bar{x} \in A \cap B$.

Proof. By [14, Proposition 5], $\{A, B\}$ has the Jameson's property, which is equivalent to linear regularity for closed convex cones by [14, Theorem 10]. Since every subspace in $\mathcal{E}$ is a closed convex cone, the result follows.

Lemma 4.7.2 can be also found in [10, Theorem 5.5.4(v)]. While the first of these statements is not surprising at all, since subspaces are convex sets, the second is not a direct result of the tools introduced in this thesis. Using both results, we deduce the following convergence statement.

Corollary 4.7.3 (global convergence for subspaces and consistent feasibility). Let $\lambda \in(0,1)$, and let $\bar{x}$ be a point in Fix $T_{A, B}^{\lambda}$. Moreover, let $A, B \subset \mathcal{E}$ be subpaces with $A \cap B \neq \emptyset$, and therefore Fix $T_{A, B}^{\lambda}=A \cap B$. Then $\{g\}:=P_{B} \bar{x}-P_{A} P_{B} \bar{x}=0$ and the sequence $\left(x^{k}\right)_{k \in \mathbb{N}}$ generated by $x^{k+1} \in T_{A, B}^{\lambda} x^{k}$ seeded by a point $x^{0} \in B$ satisfies

$$
\operatorname{dist}\left(x^{k+1}, \text { Fix } T_{A, B}^{\lambda}\right) \leq c \operatorname{dist}\left(x^{k}, \text { Fix } T_{A, B}^{\lambda}\right) \quad(\forall k \in \mathbb{N})
$$

for

$$
c:=\sqrt{1-\frac{\lambda^{2}}{\kappa^{2}}}<1,
$$


with $\kappa$ being the modulus of linear regularity. Consequently, $\operatorname{dist}\left(x^{k}, \tilde{x}\right) \rightarrow 0$ for some $\tilde{x} \in \mathrm{Fix} T_{A, B}^{\lambda}$ at least $R$-linearly with rate $c<1$. If Fix $T_{A, B}^{\lambda}$ is a singleton, then convergence is Q-linear.

Proof. The statement is a direct implication of Corollary 4.5.3 using Lemma 4.7.1 and Lemma 4.7.2.

Comparing Corollary 4.7.3 to the convergence results before (Corollary 4.5.3 and Theorem 4.4.2), we have identified a class of sets that always satisfies the assumptions of the convergence template in Theorem 3.2.6. This observation matches other results for projection methods applied to subspaces. We compare the results in the following remark.

Remark 4.7.4 (comparison of convergence rate to other projection methods). The global convergence rate stated in Corollary 4.7.3 for the method of relaxed DouglasRachford on two intersecting subspaces is bounded above by

$$
c \leq \sqrt{1-\frac{\lambda^{2}}{\kappa^{2}}}
$$

In [92] Lewis, Luke and Malick analyzed the method of alternating projections and averaged projections. They show for super-regular sets, a regularity notion that captures subspaces by Lemma 4.7.1, that the convergence rates for both algorithms are bounded above as follows

$$
\begin{aligned}
c_{M A P} & \leq 1-\frac{1}{\kappa^{2}} \\
c_{\text {averP }} & \leq 1-\frac{1}{2 \kappa^{2}},
\end{aligned}
$$

(see [92, Remark 7.5]). Comparing these three bounds, one would always prefer alternating projections before averaged projections and averaged projections before relaxed Douglas-Rachford when $\lambda<\sqrt{1-\frac{1}{4 \kappa^{2}}}$ if only these bounds are taken into consideration. Thus, the method of relaxed Douglas-Rachford does not seem to be better than the method of alternating projections for subspaces. Section 6.6 illustrates that this is not always the case for nonconvex settings. Moreover, the rate we established here is evolved from a convergence statement that included less restrictive regularity types (see for Theorem 4.4.2). Hence, by emphasizing the regularity of subspaces, we would expect a better convergence result for the method of relaxed Douglas-Rachford (compare this also to Remark 4.7.14).

When working with subspaces, there is a different notion to encounter regularity of the collection of sets $\{A, B\}$, namely angles. In particular, we are focusing here on the Friedrichs angle.

Definition 4.7.5 (Friedrichs angle [68]). The Friedrichs angle between two subspaces $A$ and $B$ is defined as the angle in $[0, \pi / 2]$ whose cosine is given by

$$
c(A, B):=\sup \left\{|\langle a, b\rangle| \mid a \in A \cap(A \cap B)^{\perp},\|a\|=1, b \in B \cap(A \cap B)^{\perp},\|b\|=1\right\} .
$$


Note that the Friedrichs angle is just one choice of many possibilities to describe the geometry of two intersecting sets. For a survey on angles between subspaces, we refer the reader to [50]. Lewis and Malick gave a generalization for manifolds in $\mathcal{E}$ depending on a specific point in the intersection and the tangent spaces at that point [93]. In the case of subpaces their definition coincides with Definition 4.7.5. Our goal is to express the modulus of linear regularity in terms of the Friedrichs angle. For this we need the following preliminary result including the notion of transversality.

Definition 4.7.6 (transversality [87, Definition 2]). Let $A, B \subset \mathcal{E}$. The collection of sets $\{A, B\}$ is transversal at a point $\bar{x} \in A \cap B$ if there exists numbers $\kappa>0, \delta>0$ and a neighborhood $U$ of $\bar{x}$ such that

$$
\begin{gathered}
\operatorname{dist}\left(x,\left(A-x_{1}\right) \cap\left(B-x_{2}\right)\right) \leq \kappa \max \left\{\operatorname{dist}\left(x, A-x_{1}\right), \operatorname{dist}\left(x, B-x_{2}\right)\right\} \\
(\forall x \in U)\left(\forall x_{1}, x_{2} \in \mathbb{B}_{\delta}\right) .
\end{gathered}
$$

We call the lower bound of all $\kappa$ that satisfy (4.25) the modulus of transversality.

Note that as compared to linear regularity as defined in Proposition 4.5.1 (i.e. subtransversality at points in the nonempty intersection), we have additional points $x_{1}$ and $x_{2}$ that translate the sets $A$ and $B$ respectively. This makes transversality a stricter property than linear regularity. The notion of transversality that is presented here was studied under several names. For a comprehensive study see $[87,88]$. For a historical commentary, see Remark 3.4.4.

Proposition 4.7 .7 (transversality and linear regularity). Let $A$ and $B$ be two subspaces in $\mathcal{E}$. Then the following hold:

(i) If the collection $\{A, B\}$ is transversal at a point $\bar{x} \in A \cap B$, then it is linear regular at $\bar{x}$ as well and the modulus of transversality is greater or equal the modulus of subtransversality.

(ii) If in addition $A+B=\mathcal{E}$, then transversality of $\{A, B\}$ at $\bar{x} \in A \cap B$ is always satisfied and the moduli of transversality and linear regularity coincide.

Proof. (i). The first part of the proposition can be found in [88, Theorem 4(i)]. We have seen in Lemma 4.7.2 that for two subspaces we always expect subtransversality, i.e. linear regularity. What is new in this context is the implication of linear regularity from a different property and the relationship of the moduli. Note that the modulus of transversality and subtransversality, respectively, are the inverse of the moduli used in [88].

(ii). For the second part assume that $A+B=\mathcal{E}$. Note that $A+B$ is closed (see for example [51, Lemma 9.36]). Therefore, we know by Lemma 4.7.2 that $\{A, B\}$ is linear regular with some modulus $\kappa_{s}$. We show next that this implies transversality of $\{A, B\}$. Since $\{A, B\}$ is linear regular at $\bar{x} \in A \cap B$ with modulus $\kappa_{s}$ we have

$$
\operatorname{dist}(x, A \cap B) \leq \kappa_{s} \max \{\operatorname{dist}(x, A), \operatorname{dist}(x, B)\}
$$


for all $x \in \mathcal{E}$. In the following let $x \in \mathcal{E}$ as well as $x_{1}, x_{2} \in \mathcal{E}$. Since $A+B=\mathcal{E}$, for every $y \in \mathcal{E}$ there exists $a \in A$ and $b \in B$ such that $y=a+b$. Applying this to $x_{1}$ and $x_{2}$ yields

$$
\begin{aligned}
\operatorname{dist}\left(x,\left(A-x_{1}\right) \cap\left(B-x_{2}\right)\right) & =\operatorname{dist}\left(x,\left(A-a_{1}-b_{1}\right) \cap\left(B-b_{2}-a_{2}\right)\right) \\
& =\operatorname{dist}\left(x,\left(A-b_{1}\right) \cap\left(B-a_{2}\right)\right),
\end{aligned}
$$

since $A$ and $B$ are both subspaces. Next, we show that

$$
\left(A-b_{1}\right) \cap\left(B-a_{2}\right)=(A \cap B)-a_{2}-b_{1} .
$$

The inclusion $\left(A-b_{1}\right) \cap\left(B-a_{2}\right) \supset(A \cap B)-a_{2}-b_{1}$ is a simple implication since $A$ and $B$ are subspaces. Thus, $A-a_{2}=A$ and respectively $B-b_{1}=B$. We show the reverse inclusion explicitly. Let $y \in\left(A-b_{1}\right) \cap\left(B-a_{2}\right)$. Therefore

$$
\begin{aligned}
y & =a-b_{1} \\
\text { and } y & =b-a_{2},
\end{aligned}
$$

for some $a \in A$ and $b \in B$. Rewriting this yields

$$
\begin{aligned}
y & =\left(a+a_{2}\right)-a_{2}-b_{1} \\
\text { and } y & =\left(b+b_{1}\right)-a_{2}-b_{1} .
\end{aligned}
$$

Since both $A$ and $B$ are subspaces, $a+a_{2} \in A$ and $b+b_{1} \in B$. Furthermore,

$$
\left(a+a_{2}\right)-a_{2}-b_{1}=y=\left(b+b_{1}\right)-a_{2}-b_{1} .
$$

From which we conclude that $a+a_{2}=b+b_{1}$. Combining these observations, we deduce that $y \in(A \cap B)-a_{2}-b_{1}$. This proves the reverse implication and therefore shows (4.27). Inserting this in (4.26) yields

$$
\operatorname{dist}\left(x,\left(A-x_{1}\right) \cap\left(B-x_{2}\right)\right)=\operatorname{dist}\left(x,(A \cap B)-a_{2}-b_{1}\right) .
$$

Rewriting the distance and using linear regularity, we deduce

$$
\begin{aligned}
\operatorname{dist}\left(x,\left(A-x_{1}\right) \cap\left(B-x_{2}\right)\right) & =\operatorname{dist}\left(x+a_{2}+b_{1},(A \cap B)\right) \\
& \leq \kappa_{s} \max \left(\operatorname{dist}\left(x+a_{2}+b_{1}, A\right), \operatorname{dist}\left(x+a_{2}+b_{1}, B\right)\right) \\
& \leq \kappa_{s} \max \left(\operatorname{dist}\left(x, A-b_{1}\right), \operatorname{dist}\left(x, B-a_{2}\right)\right) \\
& \leq \kappa_{s} \max \left(\operatorname{dist}\left(x, A-x_{1}\right), \operatorname{dist}\left(x, B-x_{2}\right)\right),
\end{aligned}
$$

which is the inequality describing transversality defined in (4.25). Denote by $\kappa_{t}$ the modulus of transversality. Then we have just shown $\kappa_{t} \leq \kappa_{s}$. By (i) we get $\kappa_{s} \leq \kappa_{t}$ and in total $\kappa_{s}=\kappa_{t}$.

Remark 4.7.8. Note that in Proposition 4.7.7(ii) the assumption $A+B=\mathcal{E}$ was crucial. Kruger, Luke and Thao gave an example of two subspaces in $\mathbb{R}^{2}$ that intersect subtransversally but not transversally [88, Example 5]. In particular, they considered the two sets $A=B=\mathbb{R} \times\{0\}$. But these two sets do not satisfy $A+B=\mathcal{E}$. This is still in line with Lemma 4.7.2. 
Lemma 4.7.9 (regularity modulus in terms of the Friedrichs angle). Let $A$ and $B$ be two subspaces in $\mathcal{E}$. If $A+B=\mathcal{E}$, the modulus of linear regularity $\kappa$ for the collection of sets $\{A, B\}$ can be described by the Friedrichs angle $c(A, B)$, namely,

$$
\kappa=\sqrt{\frac{2}{1-c(A, B)} .}
$$

Proof. We know, by Proposition 4.7.7, that the collection of sets is linear regular and transversal. Moreover, the moduli of regularity coincide. Thus, we can focus equivalently on the modulus of subtransversality instead of the modulus of linear regularity. The result then follows from [88, Proposition 8] and [88, Theorem $2(\mathrm{vi})]$.

Corollary 4.7.10. In the setting of Corollary 4.7.3 the convergence rate $c$ can be described by

$$
\sqrt{1-\frac{\lambda^{2}}{2}+\frac{\lambda^{2}}{2} c(A, B)}
$$

Proof. By Lemma 4.7.9,

$$
\kappa=\sqrt{\frac{2}{1-c(A, B)}},
$$

which is equivalent to

$$
-\frac{1}{\kappa^{2}}=-\frac{1-c(A, B)}{2} .
$$

Thus, the constant $c$ in Corollary 4.7 .3 can be reformulated as follows

$$
1>c:=\sqrt{1-\frac{\lambda^{2}}{\kappa^{2}}}=\sqrt{1-\lambda^{2} \frac{1-c(A, B)}{2}}=\sqrt{1-\frac{\lambda^{2}}{2}+\frac{\lambda^{2}}{2} c(A, B)},
$$

which proves the claim.

The motivation for the inclusion of Corollary 4.7.10 is twofold. The more apparent reason is to present the connection of the regularity of the collection of sets $\{A, B\}$ with an angle described by both subspaces. This link serves as an intuition when we should expect linear regularity. This relationship was also developed for manifolds in the context of the method of alternating projections by Lewis and Malick [93]. Deutsch and Hundal [52-54] introduced a generalization of the Friedrichs angle for more than two closed convex sets and applied their results to the method of cyclic projections. Both works demonstrate that the rate of convergence relies strongly on the angle between the sets. The other reason why we introduced the Friedrichs angle goes back to Remark 4.7.4, where we compared the rate of convergence of the relaxed Douglas-Rachford method on two subsets that is implied by Theorem 4.4.2. As we see next, this rate is not optimal. We focus on the setting when $A+B=\mathcal{E}$. In fact, we show that in this case the method of relaxed Douglas-Rachford converges R-linearly with rate at least $c(A, B)$. To prove this result, we collect the following preparatory facts. 
Lemma 4.7.11 (known but useful facts). Let $A$ and $B$ be two subspaces of $\mathcal{E}$ and denote their intersection by $M:=A \cap B$. Then

(i) The orthogonal complement $A^{\perp}$ of the subspace $A$ is again a subspace, and for every $x \in \mathcal{E}$ we can write $x=P_{A} x+P_{A^{\perp}} x$,

(ii) Fix $T_{A, B}=A \cap B \Leftrightarrow A+B=\mathcal{E}$, where $T_{A, B}$ is the mapping defined by the Douglas-Rachford algorithm in Algorithm 2.3.4,

(iii) $\left\|T_{A, B}-P_{\mathrm{Fix} T_{A, B}}\right\|=c(A, B)$,

(iv) $\left\|P_{A} P_{B}-P_{A \cap B}\right\|=c(A, B)$,

(v) $c(A, B)<1$.

Proof. (i). See for instance [51, Theorem 5.8 (1) and (2)].

(ii). See for example [19, Proposition 3.6] since $A+B$ is closed by the finite dimension of $\mathcal{E}$ (see for instance [51, Lemma 9.36]).

(iii). This was shown in [19, Theorem 4.1].

(iv). See for instance [51, Lemma 9.5(7)].

(v). This is an application of [19, Fact 2.3(i)] since $A+B$ is closed by the finite dimension of $\mathcal{E}$.

Theorem 4.7.12. Let $A$ and $B$ be two subspaces with $A \cap B \neq \emptyset$. If $A+B=\mathcal{E}$, then

$$
c(A, B) \leq\left\|T_{A, B}^{\lambda}-P_{\mathrm{Fix} T_{A, B}^{\lambda}}\right\| \leq \lambda c(A, B)+(1-\lambda),
$$

where $c(A, B)$ denotes the Friedrichs angle between $A$ and $B$ defined by Definition 4.7.5. Furthermore,

$$
\left\|T_{A, B}^{\lambda} x-P_{\mathrm{Fix} T_{A, B}^{\lambda}} x\right\| \leq(\lambda c(A, B)+(1-\lambda))\left\|x-P_{\mathrm{Fix} T_{A, B}^{\lambda}} x\right\|,
$$

for all $x \in \mathcal{E}$.

Proof. By Corollary 4.2.6, the fixed points of $T_{A, B}^{\lambda}$ are given by the intersection $A \cap B$ and hence $P_{\mathrm{Fix} T_{A, B}^{\lambda}}=P_{A \cap B}$. Using the triangle inequality, it holds for all $x \in \mathcal{E}$

$$
\begin{aligned}
\| T_{A, B}^{\lambda} x-P_{\mathrm{Fix} T_{A, B}^{\lambda} x \|} & =\left\|T_{A, B}^{\lambda} x-P_{A \cap B} x\right\| \\
& =\left\|\lambda T_{A, B} x+(1-\lambda) P_{B} x-P_{A \cap B} x\right\| \\
& =\left\|\lambda\left(T_{A, B} x-P_{A \cap B} x\right)+(1-\lambda)\left(P_{B} x-P_{A \cap B} x\right)\right\| \\
& \leq \lambda\left\|\left(T_{A, B} x-P_{A \cap B} x\right)\right\|+(1-\lambda)\left\|\left(P_{B} x-P_{A \cap B} x\right)\right\| .
\end{aligned}
$$

By Lemma 4.7.11(ii), Fix $T_{A, B}=A \cap B=$ Fix $T_{A, B}^{\lambda}$ using that $A+B=\mathcal{E}$. Thus, Lemma 4.7.11(iii) reduces to $\left\|T_{A, B}-P_{A \cap B}\right\|=c(A, B)$. Furthermore,

$$
\left\|P_{B} x-P_{A \cap B} x\right\|=\left\|P_{B}\left(x-P_{A \cap B} x\right)\right\|=\left\|P_{B} P_{(A \cap B)^{\perp}} x\right\|,
$$


implying that $\left\|\left(P_{B}-P_{A \cap B}\right)\right\| \leq 1$. Applying both result on (4.28) yields

$$
\left\|T_{A, B}^{\lambda} x-P_{\mathrm{Fix} T_{A, B}^{\lambda}} x\right\| \leq \lambda c(A, B)\|x\|+(1-\lambda)\|x\|,
$$

which proves that

$$
\left\|T_{A, B}^{\lambda}-P_{\mathrm{Fix} T_{A, B}^{\lambda}}\right\| \leq \lambda c(A, B)+(1-\lambda) .
$$

On the other hand,

$$
\begin{aligned}
& T_{A, B}^{\lambda} x-P_{\mathrm{Fix} T_{A, B}^{\lambda}} x \\
& =\lambda\left(P_{A} P_{B} x+P_{A^{\perp}} P_{B^{\perp}} x\right)+(1-\lambda) P_{B} x-P_{A \cap B} x \\
& =\lambda\left(P_{A} P_{B} x+P_{A^{\perp}} P_{B^{\perp}} x\right)+(1-\lambda)\left(P_{A} P_{B} x+P_{A^{\perp}} P_{B} x\right)-P_{A \cap B} x \\
& =\lambda P_{A} P_{B} x+(1-\lambda) P_{A} P_{B} x-P_{A \cap B} x+\lambda P_{A^{\perp}} P_{B^{\perp}} x+(1-\lambda) P_{A^{\perp}} P_{B} x \\
& =\underbrace{P_{A} P_{B} x-P_{A \cap B} x}_{\in A}+\underbrace{\lambda P_{A^{\perp}} P_{B^{\perp}} x+(1-\lambda) P_{A^{\perp}} P_{B} x}_{\in A^{\perp}} .
\end{aligned}
$$

Using Lemma 4.7.11(i), this implies that

$$
\begin{aligned}
\left\|T_{A, B}^{\lambda} x-P_{\text {Fix } T_{A, B}^{\lambda}} x\right\|^{2} & =\left\|P_{A} P_{B} x-P_{A \cap B} x\right\|^{2}+\left\|\lambda P_{A^{\perp}} P_{B^{\perp}} x+(1-\lambda) P_{A^{\perp}} P_{B} x\right\|^{2} \\
& \geq\left\|P_{A} P_{B} x-P_{A \cap B} x\right\|^{2}
\end{aligned}
$$

From which we deduce

$$
\left\|T_{A, B}^{\lambda} x-P_{\text {Fix } T_{A, B}^{\lambda}}\right\| \geq\left\|P_{A} P_{B}-P_{A \cap B}\right\|=c(A, B),
$$

by using Lemma 4.7.11(iv). Combining (4.30) and (4.29) yields

$$
c(A, B) \leq\left\|T_{A, B}^{\lambda}-P_{\text {Fix } T_{A, B}^{\lambda}}\right\| \leq \lambda c(A, B)+(1-\lambda) .
$$

Using (4.31), we deduce by [20, Lemma 3.14(1)]

$$
\left\|T_{A, B}^{\lambda} x-P_{\mathrm{Fix} T_{A, B}^{\lambda}} x\right\| \leq(\lambda c(A, B)+(1-\lambda))\left\|x-P_{\mathrm{Fix} T_{A, B}^{\lambda}} x\right\|,
$$

for all $x \in \mathcal{E}$, which concludes the proof.

Corollary 4.7.13 (linear convergence of $T_{A, B}^{\lambda}$ with $A+B=\mathcal{E}$ ). Let $A, B \subset$ Euclid be subspaces, then $\left(T_{A, B}^{\lambda}\right)^{n} x \rightarrow P_{A \cap B} x$. If $A+B=\mathcal{E}$, then convergence is at least $Q$-linear with rate $\lambda c(A, B)+(1-\lambda)<1$.

Proof. By [15, Corollary 5.16 and Proposition 5.27], we deduce $\left(T_{A, B}^{\lambda}\right)^{n} x \rightarrow P_{A \cap B} x$ since $A, B$ are subspaces, which implies that $T_{A, B}^{\lambda}$ is linear, and $T_{A, B}^{\lambda}$ is nonexpansive by Theorem 4.1.2 and Lemma 4.7.1. $T_{A, B}^{\lambda}$ is firmly nonexpansive, which we deduce by Proposition 3.3.12(ii), since $A, B$ are convex and thus $\epsilon=0$. Since $A+B=\mathcal{E}$, we can apply Theorem 4.7.12 and get by Lemma 4.7.11(v) Q-linear convergence with rate $\lambda c(A, B)+(1-\lambda)$ since $c(A, B)<1$. 
Remark 4.7.14 (improved convergence rate for subspaces). By comparing the results in Corollary 4.7.3 and Corollary 4.7.13, we deduce that the rate

$$
\lambda c(A, B)+(1-\lambda)
$$

estimated in the special case of two subspaces $A$ and $B$ such that $A+B=\mathcal{E}$ is always better than the expression

$$
\sqrt{1-\frac{\lambda^{2}}{2}+\frac{\lambda^{2}}{2} c(A, B)}
$$

regardless of the choice of the parameter $\lambda \in(0,1)$. Thus, we expect in the special case of two intersecting subspaces $A$ and $B$ with $A+B=\mathcal{E}$ that the convergence rate is not worse than $\lambda c(A, B)+(1-\lambda)$. According to a study by Bauschke et al. [19], the Douglas-Rachford method on subspaces converges linear with rate not worse than $c(A, B)$. Considering the relaxed Douglas-Rachford method as a convex relaxation between Douglas-Rachford and some projection $\left(\left\|P_{B}\right\| \leq 1\right.$ for a subspace $B$ ), the result in Corollary 4.7.13 is not surprising. Moreover, Bauschke et al. are not restricted to a setting with $A+B=\mathcal{E}$. They also do their convergence analysis in a Hilbert space instead of a finite-dimensional Euclidean space. We decided to stay finite-dimensional to be consistent with the chapters before. Note that Corollary 4.7.13 and its auxiliary results are also true in Hilbert spaces with appropriate changes, such as $A+B$ being closed for two closed subspaces. In Hilbert space, we either have to require one of the subspaces to be finite-dimensional, or $\overline{A+B}=\mathcal{E}$ (compare this to [51, Lemma 9.36] and [19, Fact 2.3]).

Example 4.7.15 (two lines in the Euclidean plane $\mathbb{R}^{2}$ ). We close this section with a small example illustrating our result in Corollary 4.7.13. In fact we consider two lines in $\mathbb{R}^{2}$, namely,

$$
\begin{aligned}
B & =\mathbb{R} \times\{0\} \\
\text { and } A & =R_{\theta}(B),
\end{aligned}
$$

where $R_{\theta}$ is the (counter-clockwise) rotator defined by

$$
R_{\theta}:=\left(\begin{array}{cc}
\cos (\theta) & -\sin (\theta) \\
\sin (\theta) & \cos (\theta)
\end{array}\right), \quad(\forall \theta \in(0, \pi / 2]) .
$$

Then, by following [19, Section 5],

$$
A \cap B=\{0\} \quad \text { and } \quad c(A, B)=\cos (\theta),
$$

as well as

$$
P_{B}=\left(\begin{array}{ll}
1 & 0 \\
0 & 0
\end{array}\right), \quad P_{A}=\left(\begin{array}{cc}
\cos ^{2}(\theta) & \sin (\theta) \cos (\theta) \\
\sin (\theta) \cos (\theta) & \sin ^{2}(\theta)
\end{array}\right)
$$

and

$$
\frac{1}{2}\left(R_{A} R_{B}+I d\right)=\cos (\theta) R_{\theta}
$$




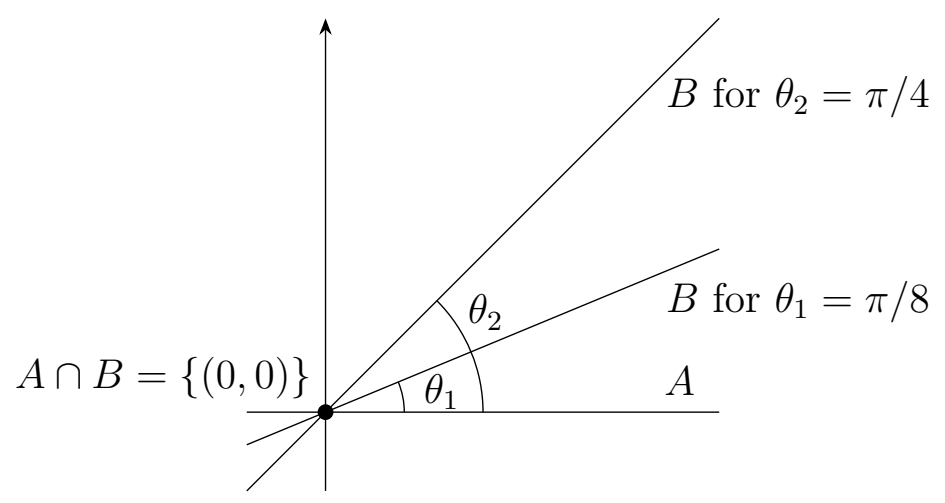

Figure 4.12: Illustration of the sets $A$ and $B$ in Example 4.7.15.

We thus deduce

$$
T_{A, B}^{\lambda}=\lambda \cos (\theta) R_{\theta}+(1-\lambda)\left(\begin{array}{ll}
1 & 0 \\
0 & 0
\end{array}\right)
$$

and expect a convergence rate at least $\lambda \cos (\theta)+(1-\lambda)$ by Corollary 4.7.13. To calculate

$$
\left\|T_{A, B}^{\lambda}-P_{A \cap B}\right\|=\left\|T_{A, B}^{\lambda}\right\|,
$$

we take $z=(x, y) \in \mathbb{R}^{2}$ with $\|z\|=1$. Then,

$$
\begin{aligned}
&\left\|T_{A, B}^{\lambda} z\right\|^{2} \\
&=\left\|\lambda \cos (\theta) R_{\theta} z+(1-\lambda)\left(\begin{array}{ll}
1 & 0 \\
0 & 0
\end{array}\right) z\right\|^{2} \\
&=\left\|\lambda \cos (\theta)\left(\begin{array}{cc}
\cos (\theta) x & -\sin (\theta) y \\
\sin (\theta) x & \cos (\theta) y
\end{array}\right)+(1-\lambda)\left(\begin{array}{l}
x \\
0
\end{array}\right)\right\|^{2} \\
&=(\lambda \cos (\theta)(\cos (\theta) x-\sin (\theta) y)+(1-\lambda) x)^{2}+(\lambda \cos (\theta)(\sin (\theta) x+\cos (\theta) y))^{2} \\
&=\lambda^{2} \cos ^{2}(\theta)\left(\cos ^{2}(\theta) x^{2}+\sin ^{2}(\theta) y^{2}\right)+(1-\lambda)^{2} x^{2} \\
&+2 \lambda(1-\lambda) x \cos (\theta)(\cos (\theta) x-\sin (\theta) y)+\lambda^{2} \cos ^{2}(\theta)\left(\sin ^{2}(\theta) x^{2}+\cos ^{2}(\theta) y^{2}\right) \\
&+2 \lambda^{2} \cos ^{3}(\theta) \sin (\theta) x y \\
&=\lambda^{2} \cos ^{2}(\theta)+(1-\lambda)^{2} x^{2}+2 \lambda(1-\lambda) x \cos (\theta)(\cos (\theta) x-\sin (\theta) y) \\
&+2 \lambda^{2} \cos ^{3}(\theta) \sin (\theta) x y,
\end{aligned}
$$

since $\sin ^{2}+\cos ^{2}=1$ and $x^{2}+y^{2}=\|z\|^{2}=1$. By $\theta \in(0, \pi, 2]$, we deduce that both $\sin (\theta) \geq 0$ and $\cos (\theta) \geq 0$. Thus, $\left\|T_{A, B}^{\lambda} z\right\|^{2}$ takes it largest value when we eliminate the only negative term in the expression. Hence, $y=0$ and $x=1$, which yields

$$
\left\|T_{A, B}^{\lambda}\right\|^{2}=\lambda^{2} \cos ^{2}(\theta)+(1-\lambda)^{2}+2 \lambda(1-\lambda) \cos ^{2}(\theta),
$$

and therefore,

$$
\left\|T_{A, B}^{\lambda}\right\|=\sqrt{\lambda^{2} \cos ^{2}(\theta)+(1-\lambda)^{2}+2 \lambda(1-\lambda) \cos ^{2}(\theta)} .
$$


This result is in line with Corollary 4.7.13, since the convergence rate, as indicated by [20, Lemma 3.14(1)] and used in the proof of Theorem 4.7.12, is described by

$$
\begin{aligned}
\left\|T_{A, B}^{\lambda} x-P_{\mathrm{Fix} T_{A, B}^{\lambda}} x\right\| & \leq\left\|T_{A, B}^{\lambda}-P_{\mathrm{Fix} T_{A, B}^{\lambda}}\right\|\left\|x-P_{\mathrm{Fix} T_{A, B}^{\lambda}} x\right\| \\
& =\left\|T_{A, B}^{\lambda}\right\|\left\|x-P_{\mathrm{Fix} T_{A, B}^{\lambda}} x\right\|,
\end{aligned}
$$

for all $x \in \mathcal{E}$. 



\section{CHAPTER 5}

\section{Cyclic Relaxed Douglas-Rachford}

The relaxed Douglas-Rachford method is limited to 2-set feasibility. As soon as more than two sets are involved, a different approach is needed. One way to do so is Pierra's product space trick presented in Section 2.3. By lifting the original sets to a product space, the $m$-set feasibility problem is reduced to a 2 -set feasibility problem. Nevertheless, this approach has its drawbacks such as the increased dimension as discussed in Section 2.3. A different idea is to cyclically compose $m$ mappings that act individually on one or two sets. A simple example for this approach is the method of cyclic projections, a more sophisticated one is the method of cyclic Douglas-Rachford by Borwein and Tam (see for instance [32,33]). This section analyzes the cyclic relaxed Douglas-Rachford as defined in Algorithm 2.3.6. That is, given some point $x^{0} \in \mathcal{E}$, a parameter $\lambda \in(0,1)$ and a finite collection of sets $\left\{\Omega_{0}, \Omega_{1}, \ldots, \Omega_{m}\right\}$, we define the relaxed Douglas-Rachford operator for two convex sets $\Omega_{j}, \Omega_{i}$ for $i, j \in\{1,2, \ldots, m\}$ similar as in Algorithm 2.3.5 to be

$$
T_{i, j}^{\lambda}:=T_{\Omega_{i}, \Omega_{j}}^{\lambda}=\frac{\lambda}{2}\left(R_{\Omega_{j}} R_{\Omega_{i}}+\mathrm{Id}\right)+(1-\lambda) P_{\Omega_{i}} .
$$

The cyclic relaxed Douglas-Rachford operator is then defined by

$$
T_{[1 \ldots m]}^{\lambda}:=T_{m, 1}^{\lambda} \circ \cdots \circ T_{2,3}^{\lambda} \circ T_{1,2}^{\lambda} .
$$

The cyclic relaxed Douglas-Rachford method generates a sequence $\left(x^{k}\right)_{k \in \mathbb{N}}$ satisfying $x^{k+1} \in T_{[1 \ldots m]}^{\lambda} x^{k}$. This method was introduced in [103] by Luke, Sabach and Teboulle. The authors discussed the algorithm only in the context of a numerical comparison to other projection methods indicating a good numerical performance of it, and did not present a detailed theoretical discussion, as we aim to give here. For $\lambda=0$, the method of cyclic relaxed Douglas-Rachford coincides with the cyclic Douglas-Rachford algorithm. If $\lambda=1$, the algorithm simplifies to cyclic projections. We study the general convergence behavior of $T_{[1 \ldots m]}^{\lambda}$ in Section 5.1 and establish an explicit description of its fixed points for the case of 2 closed and convex sets in Section 5.2. Preliminary results for the analysis of the fixed points of the $m$-set cyclic relaxed Douglas-Rachford method $(m \geq 2)$ for inconsistent feasibility 
on subspaces are presented in Section 5.3. Note that although the analysis in the subsequent sections was made in a Euclidean space $\mathcal{E}$, the results used and statements shown are not restricted to finite dimensions. Hence, the complete chapter Chapter 5 can be read as if we work on a, possibly infinite dimensional, Hilbert space $\mathcal{H}$. In this case we have to take care of distinguishing between weak and strong convergence. We decided to be consistent with the previous chapters, and keep $\mathcal{E}$ as the working space.

Parts of the subsequent chapter have its origins in discussions and joint work with Matthew K. Tam or are published in the paper [102] by Luke, Tam and the present author.

\subsection{General Convergence Analysis}

When analyzing convergence of an iterative method defined by some mapping, the classical statements rely on the corresponding fixed point set (see also Theorem 3.2.1). The same is true for compositions of operators. Given that the composition has a nonempty fixed point set, we only have to ensure that the individual operators are averaged to obtain convergence. The following theorem was originally formulated for Hilbert spaces and guarantees weak convergence. Since strong and weak convergence coincide in Euclidean spaces, we get the following version.

Theorem 5.1.1 ([15, Theorem 5.22]). Let $\alpha_{1}, \alpha_{2}, \ldots, \alpha_{m}$ be real numbers in $(0,1)$. Suppose $T_{1}, T_{2}, \ldots, T_{m}$ are nonexpansive operators, such that each $T_{i}$ is $\alpha_{i}$-averaged for $i \in\{1,2, \ldots, m\}$, and Fix $\left(T_{1} T_{2} \cdots T_{m}\right) \neq \emptyset$. Let $x^{0} \in \mathcal{E}$ and set

$$
(\forall n \in \mathbb{N}) \quad x^{n+1}=T_{m} \cdots T_{1} x^{n} .
$$

Then $x^{n}-\left(T_{m} \cdots T_{1}\right) x^{n} \rightarrow 0$ and there exists points

$$
y_{1} \in \operatorname{Fix}\left(T_{m} \cdots T_{1}\right), y_{2} \in \operatorname{Fix}\left(T_{1} T_{m} \cdots T_{2}\right), \cdots, y_{m} \in \operatorname{Fix}\left(T_{m-1} \cdots T_{1} T_{m}\right),
$$

such that

$$
\begin{aligned}
x^{n} & \rightarrow y_{1}=T_{m} y_{m}, \\
T_{1} x^{n} \rightarrow y_{2} & =T_{1} y_{1}, \\
T_{2} T_{1} x^{n} \rightarrow y_{3} & =T_{2} y_{2}, \\
& \vdots \\
T_{m-2} \ldots T_{1} x^{n} \rightarrow y_{m-1} & =T_{m-2} y_{m-2}, \\
T_{m-1} T_{m-2} \ldots T_{1} x^{n} \rightarrow y_{m} & =T_{m-1} y_{m-1} .
\end{aligned}
$$

As shown next, the operator we focus on is averaged since it is a composition of firmly nonexpansive operators if the sets are closed and convex. 
Lemma 5.1.2. Let $C_{1}, C_{2} \ldots, C_{m}$ be closed convex sets in $\mathcal{E}$. Then $T_{i, i+1}^{\lambda}$ is firmly nonexpansive and $T_{[1 \ldots m]}^{\lambda}$ is $\frac{m}{m+1}$-averaged.

Proof. We start proving that each $T_{i, i+1}$ is firmly nonexpansive. By Remark 3.3.13, we know that reflections are nonexpansive, which implies that $\frac{1}{2}\left(R_{i+1} R_{i}+\mathrm{Id}\right)$ is firmly nonexpansive by Proposition 3.1.7. Since the projection onto closed convex sets is firmly nonexpansive (see Remark 3.3.13) and the convex combination of firmly nonexpansive operators is again firmly nonexpansive (see Proposition 3.1.7), we get that each $T_{i, i+1}^{\lambda}$ is firmly nonexpansive. The averagedness of $T_{[1,2, \ldots, m]}^{\lambda}$ is an application of Combettes and Yamada's result on compositions of averaged operators in [47]. By [47, Proposition 2.5] $T_{[1 \ldots m]}^{\lambda}$ is averaged with constant

$$
\frac{1}{1+\frac{1}{\sum_{i=1}^{m} \frac{1 / 2}{1-1 / 2}}}=\frac{1}{1+\frac{1}{m}}=\frac{m}{m+1},
$$

which proves the claim.

Applying Theorem 5.1.1, we get that, if Fix $T_{[1,2, \ldots, m]}^{\lambda} \neq \emptyset$, then the cyclic relaxed Douglas-Rachford method converges. The question of convergence thus reduces to a question about the fixed point set.

Proposition 5.1.3. Let $\lambda \in(0,1)$ and let $C_{1}, C_{2}, \ldots, C_{m}$ be closed convex subsets of $\mathcal{E}$ with $\cap_{i=1}^{m} C_{i} \neq \emptyset$. Then

$$
\text { Fix } T_{[1 \ldots m]}^{\lambda}=\cap_{i=1}^{m} C_{i}
$$

Proof. Applying Corollary 4.2.6, we deduce

$$
\cap_{i=1}^{m} \operatorname{Fix} T_{i, i+1}^{\lambda}=\cap_{i=1}^{m}\left(C_{i} \cap C_{i+1}\right)=\cap_{i=1}^{m} C_{i} \neq \emptyset
$$

where $T_{m, m+1}^{\lambda}:=T_{m, 1}^{\lambda}$ and $C_{m+1}:=C_{1}$. Using [15, Corollary 4.37], yields

$$
\text { Fix } T_{[1 \ldots m]}^{\lambda}=\cap_{i=1}^{m} \text { Fix } T_{i, i+1}^{\lambda},
$$

since by Lemma 5.1.2 $T_{i, i+1}^{\lambda}$ is averaged for each $k=1,2, \ldots, m$, which concludes the proof.

Using Proposition 5.1.3, convergence to a point in the intersection $\cap_{i=1}^{m} C_{i}$ is guaranteed if the intersection is nonempty.

Corollary 5.1.4. Let $C_{1}, C_{2}, \ldots, C_{m} \subset \mathcal{E}$ be closed and convex and their intersection nonempty, i.e. $\cap_{i=1}^{m} C_{i} \neq \emptyset$. Then, for any starting point $x^{0} \in \mathcal{E}$, the sequence defined by

$$
x^{k+1}=T_{[1 \ldots m]}^{\lambda} x^{k} \quad(\forall n \in \mathbb{N})
$$

converges to a point $x$ such that

$$
x \in C=\cap_{i=1}^{m} C_{i},
$$

. If the $\mathcal{E}$ is a Hilbert space we get additionally that $\left(x^{k}-x^{k+1}\right)_{k \in \mathbb{N}}$ converges to 0 . 
Proof. Combining Lemma 5.1.2 and Proposition 5.1.3, we deduce that $T_{i, i+1}^{\lambda}$ is an averaged mapping with $\cap_{i=1}^{m}$ Fix $T_{i, i+1}^{\lambda}=\cap_{i=1}^{m} C_{i} \neq \emptyset$. The result thus follows as an application from [15, Proposition 5.15].

Remark 5.1.5. Note that the only assumptions in Theorem 5.1.1 were averagedness of the mappings $T_{i}$ and nonemptyness of the fixed point set. Hence, a similar analysis can be made for other cyclic variants of the (relaxed) Douglas-Rachford method. In [33], this was shown for the cyclic Douglas-Rachford algorithm by Borwein and Tam. Bauschke, Noll and Phan [25] used this idea to analyze the cyclically anchored Douglas-Rachford algorithm (CADRA).

When the intersection of the sets $C_{i}$ is empty, i.e. $\cap_{i=1}^{m}=\emptyset$, the situation is more delicate. Unfortunately, this case is common in a lot of applications. Therefore, the next section is dedicated to the analysis of the fixed points of $T_{[1,2, \ldots, m]}$ in a, possibly, inconsistent setting.

Remark 5.1.6 (local convergence analysis). As mentioned in [103], we believe that a local convergence analysis is possible using the framework given in [105]. We used this idea before, in Chapter 4, to prove local linear convergence of the relaxed Douglas-Rachford method. As seen there, the actual proof of convergence, in contrast to the framework, can be quite tricky and may needs auxiliary sets to show the behavior of the involved sequence. Therefore, we omit a proof for the cyclic relaxed Douglas-Rachford method using the same strategy as in Chapter 4 here.

\subsection{Fixed Points for Two Convex Sets}

In order to analyze the convergence behavior of the cyclic relaxed Douglas-Rachford method, we rely on a description of the fixed points of the original algorithm. To do so, we recall the definition of the set $E$ (respectively $F$ ), which denotes the points in $A$ (respectively $B$ ) that are closest to $B$ (respectively $A$ ) as introduced in (4.8). Moreover,

$$
g:=P_{\overline{B-A}}(0)
$$

stands for the displacement vector (or gap), between the sets $A$ and $B$ as introduced in Section 4.2. Furthermore, recall Corollary 4.2 .6 about the fixed points of relaxed Douglas-Rachford for convex sets.

Corollary 4.2.6 (fixed points for closed convex sets [97, Theorem 2.2]). Let $A$ and $B$ be two closed convex subsets of $\mathcal{E}$ and $\lambda \in(0,1)$. Then

$$
\operatorname{Fix} T_{A, B}^{\lambda}=F-\frac{\lambda}{1-\lambda} g .
$$

In particular, if $A \cap B \neq \emptyset$, then Fix $T_{A, B}^{\lambda}=A \cap B$. 


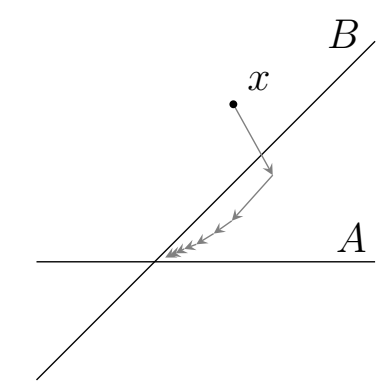

(a) Two intersecting lines.

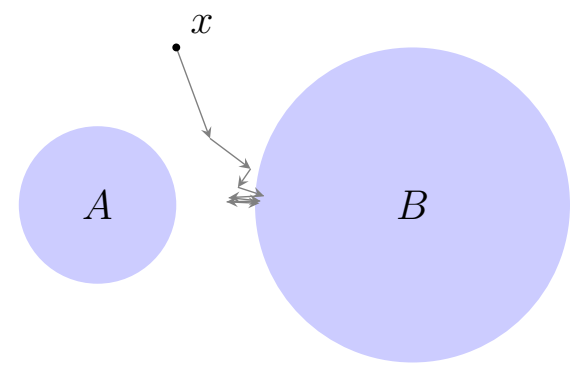

(b) Two nonintersecting balls.

Figure 5.1: Illustration of cyclic relaxed DR $(\lambda=0.4)$ applied to two affine sets $A=\mathbb{R} \times\{0\}$ and $B=\left\{x \in \mathbb{R}^{2} \mid x_{1}=x_{2}\right\}$ and applied to two balls $A=\left\{x \in \mathbb{R}^{2} \mid x_{1}^{2}+x_{2}^{2} \leq 1\right\}$ and $B=\left\{x \in \mathbb{R}^{2} \mid\left(x_{1}^{2}-4\right)+x^{2} \leq 4\right\}$ with initial point $x=(1,2) \in \mathbb{R}^{2}$ showing 4 iterations each. Each arrow represents a 2 -set relaxed Douglas-Rachford iteration.

The subsequent sections take a closer look at the fixed points of the cyclic relaxed Douglas-Rachford method in the 2-set case and the many-set case. Different statements can be made by restricting the properties of the involved sets. To develop an intuition for the behavior of the cyclic relaxed Douglas-Rachford method and its fixed points, we begin our analysis with the simplest representative case. In particular, we consider 2 closed convex sets $A, B \subset \mathcal{E}$, with possibly empty intersection. For two sets $A$ and $B$, the corresponding cyclic relaxed Douglas-Rachford method becomes

$$
\begin{aligned}
T_{[A B]}^{\lambda} & :=T_{B, A}^{\lambda} T_{A, B}^{\lambda} \\
& =\left(\frac{\lambda}{2}\left(R_{A} R_{B}+\mathrm{Id}\right)+(1-\lambda) P_{B}\right)\left(\frac{\lambda}{2}\left(R_{B} R_{A}+\mathrm{Id}\right)+(1-\lambda) P_{A}\right),
\end{aligned}
$$

where $\lambda \in(0,1)$. Even in the 2-set case the cyclic version does not coincide with the classical relaxed Douglas-Rachford because of the sequential definition. As was the case in Corollary 4.2.6 the displacement vector between the sets $A$ and $B$ is of great importance for the description of the fixed points of the cyclic relaxed Douglas-Rachford method. In fact, we show that the fixed points of the cyclic relaxed Douglas-Rachford algorithm are points in $A$ shifted by some scaled displacement vector $g$. For the proof, we require the following preliminary results.

Proposition 5.2.1 (Geometry of two sets). The following assertions hold.

(i) $\|g\|=d(A, B)$, and $E+g=F$.

(ii) $E=\operatorname{Fix}\left(P_{A} P_{B}\right)=A \cap(B-g)$, and $F=\operatorname{Fix}\left(P_{B} P_{A}\right)=B \cap(A+g)$.

(iii) Let $\gamma>0$. Then

$$
P_{B} e=P_{F} e=e+\gamma g \quad(\forall e \in E),
$$


and

$$
P_{A} f=P_{E} f=f-\gamma g \quad(\forall f \in F) .
$$

Proof. (i) and (ii). See for instance [11, Fact 1.1(i)-(ii)].

(iii). We only prove the first equality (the proof of the second is performed similarly). Let $\lambda>0$ and $e \in E$. By [11, Fact 1.1(iii)], we have $P_{B} e=P_{F} e=e+g$. Thus, for any $f \in F$, the characterization of $P_{F}$ provided by Proposition 2.2.1(ii) yields

$$
\begin{aligned}
\left\langle(e+\gamma g)-P_{F} e, f-P_{F} e\right\rangle= & \left\langle(e+g)-P_{F} e, f-P_{F} e\right\rangle \\
& +(1-\gamma)\left\langle-g, f-P_{F} e\right\rangle \\
= & \left\langle(e+g)-P_{F} e, f-P_{F} e\right\rangle \\
& +(1-\gamma)\left\langle e-P_{F} e, f-P_{F} e\right\rangle \\
\leq & 0+(1-\gamma) 0=0 .
\end{aligned}
$$

This shows that $P_{F}(e+\gamma g)=P_{F} e$, from which the result follows.

Lemma 5.2.2. Suppose $E \neq \emptyset$ and $\lambda \in(0,1)$. Then $E+\frac{1-\lambda}{1+\lambda} g \subseteq$ Fix $T_{[A B]}^{\lambda}$.

Proof. Fix $e \in E$, denote $x:=e+\frac{1-\lambda}{1+\lambda} g$ and denote $f:=e+g$. Applying Proposition 5.2.1(iii), yields $f \in F, P_{A} x=f$ and $P_{B}(f-\gamma g)=f$ for all $\gamma>0$. We therefore deduce that

$$
\begin{aligned}
R_{A} x=2 P_{B} x-x & =2 e-\left(e+\frac{1-\lambda}{1+\lambda} g\right) \\
& =e-\frac{1-\lambda}{1+\lambda} g \\
& =f-\frac{2 \lambda}{1+\lambda} g,
\end{aligned}
$$

and hence that

$$
P_{B} R_{A} x=P_{B}\left(f-\frac{2 \lambda}{1+\lambda} g\right)=f .
$$

From this it follows that

$$
\begin{aligned}
y:=T_{A, B}^{\lambda} x & =\lambda P_{B} R_{A} x+\lambda x+(1-2 \lambda) P_{A} x \\
& =\lambda f+\lambda\left(e+\frac{1-\lambda}{1+\lambda} g\right)+(1-2 \lambda) e \\
& =\lambda f+\lambda\left(f-g+\frac{1-\lambda}{1+\lambda} g\right)+(1-2 \lambda)(f-g) \\
& =f-\frac{1-\lambda}{1+\lambda} g .
\end{aligned}
$$


An analogous argument with reversed roles of $A$ and $B$ yields

$$
T_{B, A}^{\lambda} y=e+\frac{1-\lambda}{1+\lambda} g=x .
$$

Altogether, this shows that $T_{[A B}^{\lambda}{ }^{\lambda} x=x$, from which the claim follows.

Lemma 5.2.3. Suppose $E \neq \emptyset$ and $\lambda \in(0,1)$. Let $x \in$ Fix $T_{[A B]}^{\lambda}$ and denote $y:=T_{A, B}^{\lambda} x$. Then

$$
x-y=\frac{1-3 \lambda}{1+\lambda} g .
$$

Proof. Take $e \in E$, set $x^{\prime}:=e+\frac{1-\lambda}{1+\lambda} g \subseteq$ Fix $T_{\left[\begin{array}{ll}A & B\end{array}\right]}^{\lambda}$ and denote $y^{\prime}=T_{A, B}^{\lambda} x^{\prime}$. Since $T_{A, B}^{\lambda}$ is firmly nonexpansive and $T_{B, A}^{\lambda}$ is nonexpansive, we deduce that

$$
\begin{aligned}
\left\|x-x^{\prime}\right\|^{2} & \geq\left\|(x-y)-\left(x^{\prime}-y^{\prime}\right)\right\|^{2}+\left\|y-y^{\prime}\right\|^{2} \\
& \geq\left\|(x-y)-\left(x^{\prime}-y^{\prime}\right)\right\|^{2}+\left\|x-x^{\prime}\right\|^{2},
\end{aligned}
$$

and $\left\|(x-y)-\left(x^{\prime}-y^{\prime}\right)\right\|=0$. Applying Proposition 5.2.1 to $x^{\prime}$ gives $P_{A} x^{\prime}=e$ and arguing as in (5.3) gives $P_{B} R_{A} x^{\prime}-P_{A} x^{\prime}=f-e=g$. Altogether, we have

$$
\begin{aligned}
x-y=x^{\prime}-y^{\prime} & =x^{\prime}-\lambda\left(x^{\prime}+P_{B} R_{A} x^{\prime}\right)-(1-2 \lambda) P_{A} x^{\prime} \\
& =(1-\lambda) x^{\prime}-\lambda\left(P_{B} R_{A} x^{\prime}-P_{A} x^{\prime}\right)-(1-\lambda) P_{A} x^{\prime} \\
& =(1-\lambda)\left(e+\frac{1-\lambda}{1+\lambda} g\right)-\lambda g-(1-\lambda) e \\
& =\frac{1-3 \lambda}{1+\lambda} g,
\end{aligned}
$$

which proves the result.

Lemma 5.2.4. Suppose $E \neq \emptyset$ and $\lambda \in(0,1)$. Then Fix $T_{[A B]}^{\lambda} \subset E+\frac{1-\lambda}{1+\lambda} g$.

Proof. Let $x \in$ Fix $\left.T_{[A}^{\lambda} B\right]$ and set $y:=T_{A, B}^{\lambda} x$. Let $e \in E$, set $x^{\prime}:=e+\frac{1-\lambda}{1+\lambda} g$ and $y^{\prime}:=T_{A, B}^{\lambda}$. Then by Proposition 5.2.1(iii) and Lemma 5.2.2, we have $e:=P_{A} x^{\prime}$ and $x^{\prime} \in$ Fix $T_{[A B]}^{\lambda}$. Now, on one hand, since $T_{A, B}^{\lambda}$ and $T_{B, A}^{\lambda}$ are nonexpansive, we deduce that

$$
\left\|x-x^{\prime}\right\| \leq\left\|T_{A, B}^{\lambda} x-T_{A, B}^{\lambda} x^{\prime}\right\| \leq\left\|x-x^{\prime}\right\| \Longrightarrow\left\|x-x^{\prime}\right\|=\left\|y-y^{\prime}\right\| .
$$

On the other hand, convexity of $\|\cdot\|^{2}$ and firm nonexpansivity of $P_{A}$ give

$$
\begin{aligned}
\left\|y-y^{\prime}\right\|^{2} & =\left\|\lambda\left(T_{A, B} x-T_{A, B} x^{\prime}\right)+(1-\lambda)\left(P_{A} x-P_{A} x^{\prime}\right)\right\|^{2} \\
& \leq \lambda\left\|T_{A, B} x-T_{A, B} x^{\prime}\right\|^{2}+(1-\lambda)\left\|P_{A} x-P_{A} x^{\prime}\right\|^{2} \\
& \leq \lambda\left\|x-x^{\prime}\right\|^{2}+(1-\lambda)\left[\left\|x-x^{\prime}\right\|^{2}-\left\|\left(x-P_{A} x\right)-\left(x^{\prime}-P_{A} x^{\prime}\right)\right\|^{2}\right] \\
& =\left\|x-x^{\prime}\right\|^{2}-(1-\lambda)\left\|\left(x-P_{A} x\right)-\left(x^{\prime}-P_{A} x^{\prime}\right)\right\|^{2} .
\end{aligned}
$$


Altogether, we have $\left\|\left(x-P_{A} x\right)-\left(x^{\prime}-P_{A} x^{\prime}\right)\right\|^{2}=0$ and hence

$$
x=P_{A} x+x^{\prime}-P_{A} x^{\prime}=P_{A} x+\frac{1-\lambda}{1+\lambda} g .
$$

An anolguous argument gives

$$
y=P_{B} y+y^{\prime}-P_{B} y^{\prime}=P_{B} y-\frac{1-\lambda}{1+\lambda} g .
$$

Subtracting the last two equations and applying Lemma 5.2.3, we deduce

$$
P_{B} y-P_{A} x=(y-x)+\frac{2(1-\lambda)}{1+\lambda} g=\frac{3 \lambda-1}{1+\lambda} g+\frac{2(1-\lambda)}{1+\lambda} g=g .
$$

That is to say, $\left(P_{A} x, P_{B} y\right)$ attains the minimum distance between $A$ and $B$ and, consequently, we have $P_{A} x=P_{E} x$ (and $P_{B} y=P_{F} y$ ). Substituting this back into (5.4) gives

$$
x=P_{E} x+\frac{1-\lambda}{1+\lambda} g \in E+\frac{1-\lambda}{1+\lambda} g,
$$

which proves the result.

Theorem 5.2.5. Suppose $\lambda \in(0,1)$ and assume $E \neq \emptyset$. Then

$$
\text { Fix } T_{[A B]}^{\lambda}=E+\frac{1-\lambda}{1+\lambda} g \text {. }
$$

Proof. The result follows by combining Lemma 5.2.2 and Lemma 5.2.4.

Example 5.2.6. To illustrate Fix $T_{[A B]}^{\lambda}$, we consider again the sets presented in Example 4.2.5. Note that $\lambda=0.4$. By Theorem 5.2.5 and the definitions in Example 4.2.5, the fixed point sets turn into Fix $T_{[A B]}^{\lambda}=(1.6,0) \times \mathbb{R}$ as shown in Fig. 5.2a, Fix $T_{[A B]}^{\lambda}=(1.6,0)$ for the second example as in Fig. 5.2b and Fix $T_{[A B]}^{\lambda}=(1,0)=A \cap B$ for the last case shown in Fig. 5.2c.

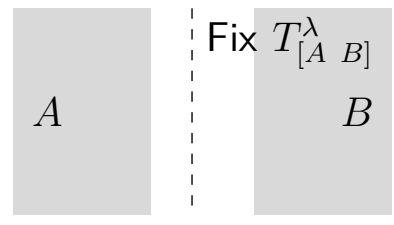

(a) Two nonintersecting sets.

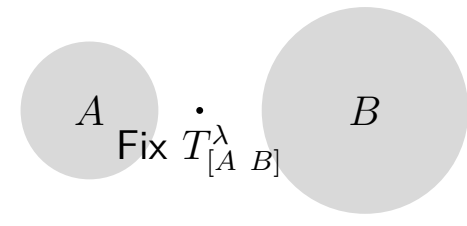

(b) Two nonintersecting balls.

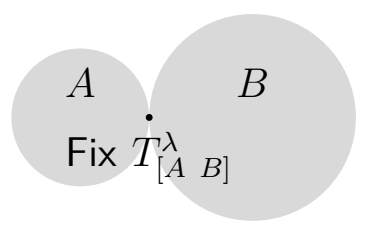

(c) Two intersecting balls.

Figure 5.2: Illustration of Fix $\left.T_{[A B}^{\lambda} B\right]$ for two closed convex sets $A$ and $B(\lambda=0.4)$.

Remark 5.2.7. Note that Theorem 5.2.5 assumes that the set of nearest points in $A$ to $B$, that is $E$, has to be nonempty. We conjecture that, if $E=\emptyset$ no fixed points of $T_{\left[\begin{array}{ll}A & B\end{array}\right]}^{\lambda}$ exist and expect that a similar dichotomy analysis can be made for the cyclic relaxed Douglas-Rachford method on two sets as for the cyclic Douglas-Rachford method in [32] by Borwein and Tam and for the method of cyclic projections in [13, Theorem 5.2.1.]. 


\subsection{Fixed Points for $m$ Subspaces}

In the section above, we were able to characterize the fixed point set of the cyclic relaxed Douglas-Rachford method on 2 closed convex sets $A$ and $B$ explicitly. The consistent $m$-set case was studied in Proposition 5.1.3. The inconsistent case remains open. While it is hard to investigate the general setting of closed sets, we are able to make some statements on (linear) subspaces. Although this is a restriction, it is nevertheless a first step to prove results on the set of fixed points in the inconsistent setting for general sets. To this end, we analyze difference vectors. Similar to the definition of the difference vectors in Section 4.3, we set for $y \in \mathcal{E}$

$$
x_{0}:=y \quad \text { and } \quad x_{i+1}:=T_{i, i+1} x_{i} \quad \forall i \in\{0, \ldots, m-1\}
$$

Then $d_{i}:=x_{i}-x_{i+1}$ for $i \in\{1, \ldots, m\}$ are referred to as difference vectors, $\left\{x_{i}\right\}_{i \in\{1, \ldots, m\}}$ is called the corresponding cycle of inner iterations of the point $y$. Note that, in contrast to Chapter 4 , the different vectors used here are elements of $\mathcal{E}$ instead of $\mathcal{E}^{4}$. Despite this conceptual difference, we decided to call both objects difference vectors.

Lemma 5.3.1. Let the sets $\left\{\Omega_{1}, \ldots, \Omega_{m}\right\} \subset \mathcal{E}$ be subspaces. Then

$$
P_{i+1} x_{i+1}=P_{i+1} P_{i} x_{i} \quad(\forall i \in\{0,1, \ldots, m-1\}) .
$$

Proof. We begin computing $P_{i+1} x_{i+1}$ by using the definition of $x_{i+1}$.

$$
\begin{aligned}
P_{i+1} x_{i+1} & =P_{i+1}\left(T_{i, i+1}^{\lambda} x_{i}\right) \\
& =P_{i+1}\left(\lambda\left(P_{i+1} R_{i} x_{i}+x_{i}\right)+(1-2 \lambda) P_{i} x_{i}\right) \\
& =\lambda P_{i+1} R_{i} x_{i}+\lambda P_{i+1} x_{i}+(1-2 \lambda) P_{i+1} P_{i} x_{i},
\end{aligned}
$$

since a projector onto a (linear) subspace is linear by Proposition 2.2.4. Using Proposition 2.2.4 a second time and applying the definition of the reflector yields

$$
\begin{aligned}
P_{i+1} x_{i+1} & =P_{i+1}\left(T_{i, i+1}^{\lambda} x_{i}\right) \\
& =\lambda P_{i+1}\left(2 P_{i} x_{i}-x_{i}\right)+\lambda P_{i+1} x_{i}+(1-2 \lambda) P_{i+1} P_{i} x_{i} \\
& =2 \lambda P_{i+1} P_{i} x_{i}-\lambda P_{i+1} x_{i}+\lambda P_{i+1} x_{i}+(1-2 \lambda) P_{i+1} P_{i} x_{i} \\
& =P_{i+1} P_{i} x_{i},
\end{aligned}
$$

which completes the proof.

Remark 5.3.2. From Lemma 5.3.1, we deduce that the projection of the inner iterations onto the corresponding sets yield the cycle of inner iterations of the fixed points for $\lambda=0$, i.e. the fixed points and its inner iterations of the corresponding 
cyclic projections mapping $P_{m} P_{m-1} \cdots P_{1}$. In particular, using Lemma 5.3.1, we obtain

$$
\begin{aligned}
P_{m} y & =P_{m} P_{m-1} x_{m-1} \\
& =P_{m} P_{m-1} P_{m-1} x_{m-2} \\
& =\cdots=P_{m} P_{m-1} \cdots P_{1} x_{1}=P_{m} P_{m-1} \cdots P_{1} P_{m} y,
\end{aligned}
$$

for $y$ and $x_{1}, x_{2}, \ldots, x_{m-1}$ defined as in (5.5).

Lemma 5.3.3. Let the sets $\left\{\Omega_{1}, \ldots, \Omega_{m}\right\}$ be subspaces. Fix $\lambda \in(0,1)$ and take $y \in$ Fix $T_{[1 \ldots m]}^{\lambda}$ and denote the corresponding cycle of inner iterations by $\left\{x_{i}\right\}_{i \in\{1, \ldots, m\}}$. Then, for any choice of the relaxation parameter $\lambda$, the fixed points lie on the line

$$
\left\{t P_{m} x_{m-1}+(1-t) P_{1} P_{m} x_{m-1} \mid t \in \mathbb{R}^{n}\right\} .
$$

Similarly, the corresponding inner iterations $x_{m-1}, x_{m-2}, \ldots, x_{1}$ lie on the lines

$$
\begin{aligned}
& \left\{t P_{m-1} x_{m-2}+(1-t) P_{m} P_{m-1} x_{m-2} \mid t \in \mathbb{R}^{n}\right\} \\
& \left\{t P_{m-2} x_{m-3}+(1-t) P_{m-1} P_{m-2} x_{m-3} \mid t \in \mathbb{R}^{n}\right\} \\
& \vdots \\
& \left\{t P_{1} y+(1-t) P_{2} P_{1} y \mid \in \mathbb{R}^{n}\right\} .
\end{aligned}
$$

Proof. Fix $\lambda \in(0,1)$ and let $\tilde{\lambda} \in(0,1)$ be a relaxation parameter different than $\lambda$. Let $\tilde{y} \in \operatorname{Fix} T_{[1 \ldots m]}^{\tilde{\lambda}}$ and $\left\{\tilde{x}_{i}\right\}_{i \in\{1, \ldots, m\}}$ the corresponding inner iterations to $\tilde{\lambda}$. Then

$$
\begin{aligned}
\tilde{y} & =\tilde{\lambda}\left(P_{1} R_{m} x_{m-1}\right)+(1-2 \tilde{\lambda}) P_{m} \tilde{x}_{m-1} \\
\text { and } \quad P_{1} \tilde{y} & =P_{1} P_{m} \tilde{x}_{m-1},
\end{aligned}
$$

as before. From Remark 5.3.2, we get that the projection of $\tilde{y}$ coincides with the fixed point of cyclic projections. But this is also true for $P_{1} y$. Therefore,

$$
P_{1} \tilde{y}=P_{1} y, \quad \text { and similarly } \quad P_{1} y=P_{1} P_{m} x_{m-1}
$$

which implies

$$
P_{1} \tilde{y}=P_{1} P_{m} x_{m-1}
$$

Thus, it remains to show that the normal cone at $P_{1} \tilde{y}$ of the set $C_{1}$ can be identified with

$$
\left\{t P_{m} x_{m-1}-t P_{1} P_{m} x_{m-1} \mid t \in \mathbb{R}^{n}\right\}
$$

To this end, note that

$$
N_{C_{1}}\left(P_{1} P_{m} x_{m-1}\right)=\text { cone }\left(P_{1}^{-1}\left(P_{1} P_{m} x_{m-1}\right)-P_{1} P_{m} x_{m-1}\right),
$$

since $C_{1}$ is a subspace and thus convex. Moreover,

$$
P_{m} x_{m-1} \in P_{1}^{-1}\left(P_{1} P_{m} x_{m-1}\right) .
$$


By Proposition 2.2.4, the projector onto a (linear) subspace is a linear operator. Thus, we deduce

$$
t P_{m} x_{m-1} \in P_{1}^{-1}\left(P_{1} P_{m} x_{m-1}\right)
$$

for $t \in \mathbb{R}$. By the same argument, one can show by contradiction that these are the only points on $P_{1}^{-1}\left(P_{1} P_{m} x_{m-1}\right)$. This implies

$$
N_{C_{1}}\left(P_{1} P_{m} x_{m-1}\right)=\left\{t P_{m} x_{m-1}-t P_{1} P_{m} x_{m-1} \mid t \in \mathbb{R}^{n}\right\} .
$$

Translating the normal cone to $P_{1} P_{m} x_{m-1}$ yields the claimed result for $y$. The claim for $x_{m-1}$ to $x_{1}$ can be proven by the same strategy.

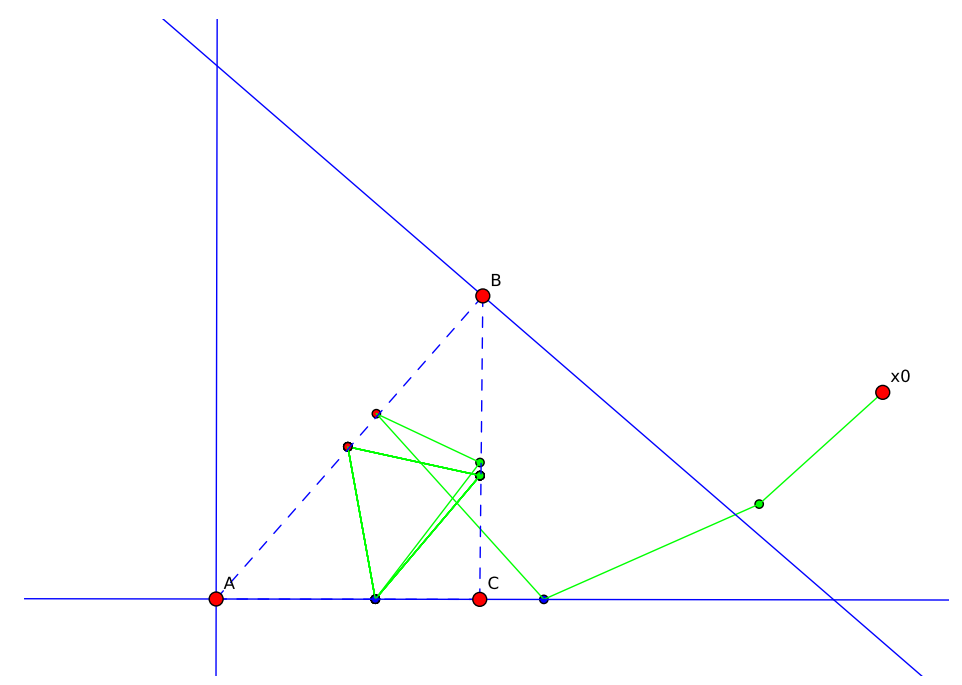

Figure 5.3: Cyclic relaxed DR $(\lambda=0.4)$ applied to three lines in $\mathbb{R}^{2}$. Shown are the iterations and the fixed points for cyclic projections $(A, B, C)$ for some initial point $x^{0}$. Each green line represents a 2-set relaxed Douglas-Rachford iteration.

Remark 5.3.4. We conjecture that this statement can be further refined. Assuming that the method of cyclic projections has fixed points, we believe that a fixed point of $T_{[1 \cdots m]}^{\lambda}$ for closed and convex sets $C_{1}, C_{2}, \ldots, C_{m}$ and its inner iterations lie in the convex combination of a corresponding fixed point of the cyclic projections algorithm and its inner iterations. Evidence for this conjecture is provided by experiments made using interactive geometry applets in Cinderella as shown in Fig. 5.3 and Fig. 5.4. On the other hand, similar results on the existence of fixed points are shown for the cyclic Douglas-Rachford method in [32]. Borwein and Tam identified the fixed points of the cyclic Douglas-Rachford algorithm with the fixed points of a cyclic projections scheme. Since the cyclic Douglas-Rachford method is an extreme case of its relaxed version, it is reasonable that the existence of fixed points of the cyclic relaxed Douglas-Rachford method depends on the fixed points of cyclic projections as well. Unfortunately, the fixed points of cyclic projections lack a description of their fixed points for more than 2 sets. Baillon, Combettes 
and Cominetti showed that, in general, there does not exists a variational characterisation of these cycles (as for example in the 2-set case) [7]. Thus, even if our conjecture regarding fixed points of cyclic relaxed Douglas-Rachford is true, we still rely on the assumption that there exist fixed points of the cyclic projection method.

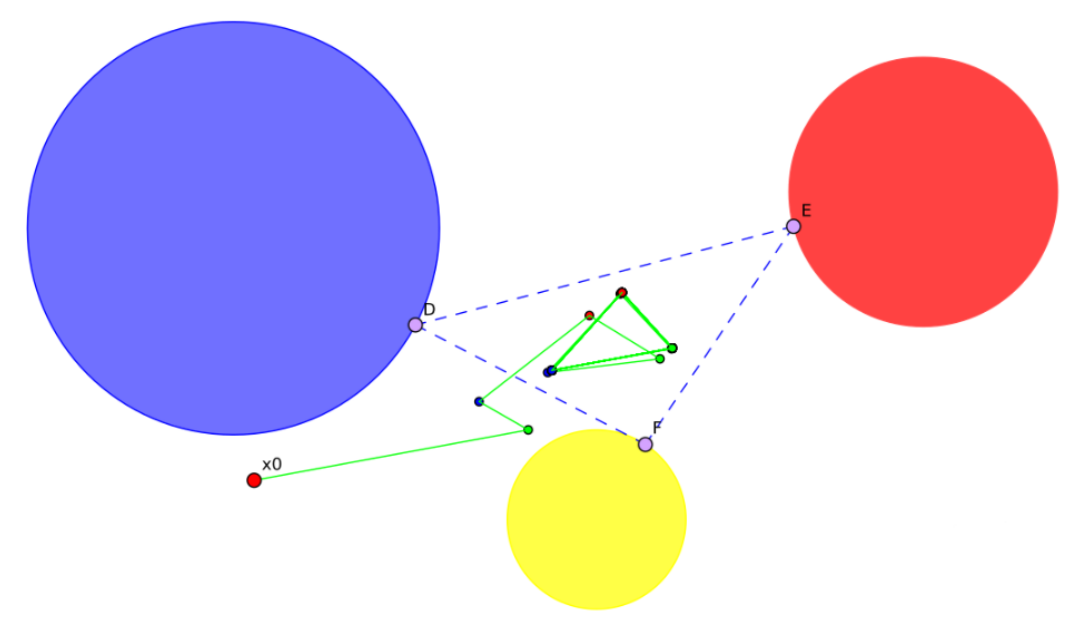

Figure 5.4: Cyclic relaxed DR $(\lambda=0.4)$ applied to three balls in $\mathbb{R}^{2}$. Shown are the iterations and the fixed points for cyclic projections $(D, E, F)$ for some initial point $x^{0}$. Each green line represents a 2-set relaxed Douglas-Rachford iteration. 


\section{CHAPTER 6}

\section{Phase Retrieval}

The phase retrieval problem is known in physics for decades ${ }^{1}$. In mathematics this problem got the scientists interest in the 1950s and since than has been a problem that produced a considerable amount of findings in various fields of mathematics. Physicists invented a diverse range of algorithms with a striking good performance to solve this problem, but couldn't explain theoretically why the performance is that good, whereas mathematicians tried to find an explanation and came up with more algorithmic ideas to create (theoretically) even better results. Acknowledging that both areas of research have produced a wide range of results, we focus here on the most prominent algorithms like the error reduction algorithm or Fienup's hybrid input output method (HIO). These algorithms, and almost all other frequently applied methods to solve the phase retrieval problem, fit into the framework of iterative projection algorithms as introduced in Section 2.3. That is, to remind the reader, given some (set-valued) mapping $T: \mathcal{E} \rightrightarrows \mathcal{E}$ composed of projectors, the iterative algorithm defines a sequence $\left(x^{k}\right)_{k \in \mathbb{N}}$ by

$$
x^{k+1} \in T x^{k} \quad(\forall k \in \mathbb{N}) .
$$

Algorithms used to solve the phase retrieval problem generate sequences of points that, as one hopes, deliver an adequate solution to the problem. Given such a sequence, convergence of it to a fixed point of $T$ is desired. Physically the convergence of the sequence $\left(x^{k}\right)_{k \in \mathbb{N}}$ to a point $x$ [...means that the energy of the residual signal $\left\|x^{k}-x\right\|^{2}$ becomes arbitrarily small as $k$ increases...] [16].

Solving the phase retrieval problem with a projection method, the mapping $T$ has to suit the physical setup of the underlying experiment. Additionally, to deduce convergence of the sequence $\left(x^{k}\right)_{k \in \mathbb{N}}$, one has to assure that regularity conditions, as discussed in Chapter 3, are satisfied. We focus here on treating the physical constraints as well as the convergence analysis of the algorithms theoretically. In other words, we treat the question, what results the experimenter can expect from its numerical data.

\footnotetext{
${ }^{1}$ One of the first references is a letter of Lord Rayleigh to A.Michelson [116].
} 
The phase retrieval problem consists in reconstructing an object given only the magnitude of the object under some transformation. Thus, the solution and the given measurements usually are elements in different spaces, but they are connected by some transformation that represents the experimental setup. Moreover, one is often given some a priori information that includes, for example, knowledge about the support or the values of the object. Common qualitative constraints, besides a support constraint, are sparsity, amplitude constraints, or nonnegativity. All this information can be represented in constraint sets, each information in an individual one. Seeking a solution to the phase retrieval problem is then nothing more but finding a solution that satisfies all the constraints. Mathematically this fits into the framework of feasibility problems.

A survey on the most prominent algorithms for the phase retrieval problem, in the context of feasibility problems, was, for instance, done by Bauschke et al. in 2002 [16] and by Marchesini in 2007 [106]. It appears that most of the algorithms relate to a mapping $T$ that consists of proximal operators as defined in (2.13). Although their description is essential to understand the problem in the context of proximal mappings, the convergence analysis given, lacks nonconvexity. A property that is often present in real-life applications - such as in the phase retrieval problem. The set of measurements is highly nonconvex, as we discuss in Section 6.3, and can thus lead to ambiguities of related projections and local minima of algorithms. Following the framework introduced by Luke, Thao and Tam in [105], we gave a proof for local R-linear convergence of the relaxed Douglas-Rachford method for nonconvex feasibility problems (see Chapter 4). Their strategy to prove convergence included two essential properties. On the one hand the regularity of the sets themselves, and on the other hand a regularity notion of the collection of all involved sets. The better the regularities of the sets and their collection, the better the (local) convergence results. Following the ideas of [16], [106] and [105], our aim here is to carry out a nonconvex convergence analysis of projection based algorithms to solve the phase retrieval problem. This is not only showing that a lot of the wellknown algorithms fit into a certain framework, but also, that we can provide a local convergence analysis of these. The most popular class of phase retrieval algorithms are projection methods. Nonetheless, there are numerous ways to investigate or solve the phase retrieval problem. Other approaches consist in using semidefinite programming and suitable relaxations (see for instance [36-38, 125]), sparsity-based algorithms (see [26,95]), Wirtinger Flow algorithms (see for instance [42]) and many others. For more references the reader is referred to the following surveys $[82,99,106,120]$.

\subsection{Phase Retrieval as a Feasibility Problem}

The phase retrieval problem consists in reconstructing a complex valued object in a plane (the object plane) from measurements of its amplitude under a unitary mapping in a plane somewhere downstream from the object plane (the image plane). 
Thus, the phase information of the measurements is missing. This problem is of great importance in various areas in physics and engineering, such as electron microscopy [57], astronomy [100], optical design and holography $[65,85]$. The problem just described can be broadly separated into two classes-near field and far field imaging. Near field imaging, as its name suggests, describes a setting where the measurements are relatively close to the object plane. Whereas in far field imaging, the measurements are relatively far from the object plane. Both settings lead to different types of unitary mappings that are involved in the phase retrieval problem. In the near field setup it is the Fresnel propagator, in the far field the Fourier transform. Fortunately, both of these mappings can be computed by coupling the Fourier transform $\mathcal{F}$ with some unitary linear mapping $\mathcal{P}$, that contains the physical details, such as magnification and translation. The image that is measured in the experiment contains discrete data like the photons counted in the image plane. Hence, the Fourier transform, which we denote by $\mathcal{F}$, is discrete, i.e. $\mathcal{F} \in \mathbb{C}^{n \times n}$. In particular, let $x=\left(x_{1}, x_{2}, \ldots, x_{n}\right) \in \mathbb{C}^{n}$, then $\mathcal{F} x=z$, where

$$
z_{j}:=\frac{1}{\sqrt{n}} \sum_{k=0}^{n-1} x_{k+1} \exp \left(-i 2 \pi \frac{(j-1) k}{n}\right) \quad(\forall j=1,2, \ldots, n) .
$$

Thus, the underlying space we consider in this chapter is mostly $\mathbb{C}^{n}$ and $\mathcal{F}$ is unitary.

Following Luke, Sabach and Teboulle in [103], the physical model of the measurements can be described by

$$
\left|\left(\mathcal{F} \cdot \mathcal{P}_{j} z\right)_{k}\right|=b_{k j}, \quad(\forall j=1,2, \ldots, m)(\forall k=1,2, \ldots, n),
$$

where $z \in \mathbb{C}^{n}$ is the complex-valued vector, that we wish to find and $m$ is the number of measurements taken. The measurements are recorded in the $b_{k j}$ 's. One image of $n$ pixels is given by a vector $b_{j} \in \mathbb{R}_{+}^{n}$, where $\mathbb{R}_{+}^{n}$ is the set of $n$-dimensional real-valued vectors with nonnegative entries. Hence, $b_{k j}$ is the $k$-th pixel of the $j$-th image, $b_{j}$, taken. We choose $\mathcal{P}_{j}$ instead of $\mathcal{P}$ to describe experiments with different settings in each of the actual measurements, as discussed for instance in $[72,100]$. Again, all mappings $\mathcal{P}_{j}$ are unitary and linear.

We model this problem as a feasibility problem. That is, given a collection of sets we want to find a point in the intersection of these, or, if this intersection is empty, a point that is close to each of the sets, i.e. an approximation. Thus, we have to formulate the phase retrieval problem with respect to sets. The most important one is the set describing the measurements as in (6.2), which we denote by

$$
M_{j}:=\left\{z \in \mathbb{C}^{n}||\left(\mathcal{F} \cdot \mathcal{P}_{j} z\right)_{k} \mid=b_{k j}, \forall k=1,2, \ldots, n\right\} .
$$

Similarly, one can use the set

$$
M_{j}^{\prime}:=\left\{z \in \mathbb{C}^{n}||(\mathcal{F} z)_{k} \mid=b_{k j}, \forall k=1,2, \ldots, n\right\}
$$


or

$$
\widehat{M}_{j}^{\prime}:=\left\{z \in \mathbb{C}^{n}|| z_{k} \mid=b_{k j}, \forall k=1,2, \ldots, n\right\} .
$$

Note that the three sets are related to each other via the following identity

$$
M_{j}=\mathcal{P}_{j}^{*} M_{j}^{\prime}=\mathcal{P}_{j}^{*} \mathcal{F}^{*} \widehat{M}_{j} \quad(\forall j=1,2, \ldots, m),
$$

where $\mathcal{P}_{j}^{*}$ and $\mathcal{F}^{*}$ denote the adjoints of $\mathcal{P}_{j}$ and $\mathcal{F}$, respectively. Using (6.3),(6.4) or (6.5) for the description of the measurement constraint however, depends on ones personal choice. Since all three sets are related to each other using unitary mappings, as seen in (6.6), there exists a unitary isomorphism between the three different underlying spaces [103].

In addition to the measurement constraint, there are certain a priori known qualitative constraints that might be added, depending on the type of experiment. This additional information about the model can be of various character. Common examples are the support of the object, real-valuedness, nonnegativity, sparsity or the information about an amplitude. We refer to these sets by the following notation.

$$
\begin{aligned}
\text { support constraint } & \mathfrak{S}:=\left\{y \in \mathbb{C}^{n} \mid y_{i}=0 \forall i \notin I\right\} \\
\text { support and real-valued constraint } & \mathfrak{S}_{r}:=\left\{y \in \mathbb{R}^{n} \mid y_{i}=0 \forall i \notin I\right\} \\
\text { support and nonnegative constraint } & \mathfrak{S}_{+}:=\left\{y \in \mathbb{R}_{+}^{n} \mid y_{i}=0 \forall i \notin I\right\} \\
\text { amplitude constraint } & A:=\left\{y \in \mathbb{C}^{n}|| y_{k} \mid=a_{k}, 1 \leq k \leq n\right\} \\
\text { sparsity constraint } & \mathcal{A}_{s}:=\left\{y \in \mathbb{R}^{n} \mid\|x\|_{0} \leq s\right\}
\end{aligned}
$$

for a set of indices $I \subset\{1,2, \ldots, n\}, a \in \mathbb{R}^{n}$ nonnegative and $s \in\{1,2, \ldots, n\}$.

Using the introduced constraint sets, we can formulate the phase retrieval problem by finding a point $x \in \mathbb{C}^{n}$ that satisfies two of the introduced sets. In particular,

$$
\text { find } x \in \cap_{j=1}^{m} M_{j} \cap \mathcal{O} \text {, }
$$

where $\mathcal{O}$ is one of the qualitative constraints in (6.7)-(6.11). We restrict our analysis in this chapter to a 2 -set feasibility problem. If one wants to include more than one of the a priori assumptions specified in (6.7)-(6.11), they can be combined in one set represented here by $\mathcal{O}$.

The problem of recovering the phase from just measurements of the objects modulus is impossible to solve uniquely. It is ill-posed. One ambiguity that arises is the shift by a global phase. If a point $x \in \cap_{j=1}^{m} \mathcal{M}_{j}$ then $\exp (i \phi) x$ for any $\phi \in \mathbb{R}$ is also a point in $\cap_{j=1}^{m} \mathcal{M}_{j}$. To see this, note the following

$$
\left(\mathcal{F} \cdot \mathcal{P}_{j}(\exp (i \phi) x)\right)_{k}=\exp (i \phi)\left(\mathcal{F} \cdot \mathcal{P}_{j}(x)\right)_{k} \quad(\forall k=1,2, \ldots,),
$$

since both $\mathcal{F}$ and $\mathcal{P}_{j}$ are linear. But not only the lack of information contributes to this property of the problem. When taking real problems, measurement errors and 
noisy data play a big role. Often the measurements indicate all different solutions that do not coincide. A solution from the provided information is then only an approximation to the actual object. Mathematically these characteristics translate into an inconsistent feasibility problem. That is, the intersection of the involved sets $M_{j}$ and $\mathcal{O}$ is empty.

\subsection{Projectors onto Constraint Sets}

Since we want to analyze projection methods for the phase retrieval problem, this section is devoted to the projectors onto the constraint sets introduced in Section 6.1. The projector onto the measurements, $M_{j}$, as defined in (6.3), can be described as follows.

Lemma 6.2.1 (projector onto measurement constraint). Let $z \in \mathbb{C}^{n}$. Then the following are equivalent for all $j \in\{1,2, \ldots, m\}$ :

$$
y \in P_{M_{j}} z \Leftrightarrow y=\mathcal{P}_{j}^{*} \mathcal{F}^{*} \widehat{y}
$$

where the entries of $\widehat{y}$ are given by

$$
\widehat{y}_{k} \in \begin{cases}b_{k j} \frac{\left(\mathcal{F} \mathcal{P}_{j} z\right)_{k}}{\left|\left(\mathcal{F} \mathcal{P}_{j} z\right)_{k}\right|}, & \text { if }\left(\mathcal{F} \mathcal{P}_{j} z\right)_{k} \neq 0 \\ \left\{b_{k j} \exp (i \phi) \mid \phi \in[0,2 \pi]\right\}, & \text { if }\left(\mathcal{F} \mathcal{P}_{j} z\right)_{k}=0\end{cases}
$$

for all $k \in\{1,2, \ldots, n\}$.

Proof. See for example [16, Example 3.6].

The sets $M_{j}$ are not convex. In the Fourier domain the set $M_{j}$ can be seen coordinatewise as spheres in $\mathbb{R}^{2}$ when all other elements are fixed (see for instance [100]). The projector of the origin onto $M_{j}$ is multi-valued, and thus leads to infinitely many phases. Thus, whenever $y_{j}=0$, any phase will be a solution. In practice one avoids such ambiguities by selecting a specific phase like $\phi=0$, which leads to $z_{j}=b_{j}$. Despite being nonconvex, the sets $M_{j}$ are closed (see for instance [16, Example 3.15]), and its projector is still easy to compute. We analyze the regularities of the involved sets in more detail in Section 6.3. The projectors onto the constraint sets are even simpler to compute than the projector onto the measurement constraint.

Lemma 6.2.2 (projectors onto object domain constraints). Let $y \in \mathbb{C}^{n}, I \subset$ $\{1,2, \ldots, n\}$ be a finite index set and $a \in \mathbb{R}^{n}$ be nonnegative. Then the projector onto the support constraint, the projector onto support and real-valued constraint 
and the projector onto the support and nonnegative constraint are, respectively, given by

$$
\begin{aligned}
& P_{\mathfrak{S}} y=z \quad \text { where } z_{j}= \begin{cases}y_{j} & \text { if } j \in I \\
0 & \text { otherwise }\end{cases} \\
& P_{\mathfrak{S}_{r}} y=z \quad \text { where } z_{j}= \begin{cases}\operatorname{Re}\left(y_{j}\right) & \text { if } j \in I \\
0 & \text { otherwise }\end{cases} \\
& P_{\mathfrak{S}_{+}} y=z \quad \text { where } z_{j}=\left\{\begin{array}{ll}
\max \left\{\operatorname{Re}\left(y_{j}\right), 0\right\} & \text { if } j \in I \\
0 & \text { otherwise }
\end{array} \quad \text { for } i=1,2, \ldots, n .\right. \\
& \text { for } i=1,2, \ldots, n \text {, } \\
& \text { for } i=1,2, \ldots, n \text {, }
\end{aligned}
$$

The projector onto the amplitude constraint is given by

$$
P_{A} y=z,
$$

where

$$
z_{j}=\left\{\begin{array}{ll}
a_{j} \frac{y_{j}}{\left|y_{j}\right|} & \text { if } z_{j} \neq 0 \\
\left\{\exp (i \phi) a_{j} \mid \phi \in[0,2 \pi]\right\} & \text { otherwise }
\end{array} \quad \text { for } i=1,2, \ldots, n .\right.
$$

Whereas the projector onto the sparsity constraint is given by

$$
P_{\mathcal{A}_{s}} y=\left\{z \in \mathbb{R}^{n} \mid z_{i}=\left\{\begin{array}{ll}
y_{i}, & j \in \mathbb{J}, \\
0, & j \notin \mathbb{J},
\end{array}\right\} \quad \text { for some } \mathbb{J} \in \mathcal{J}_{s}(y),\right.
$$

where

$$
J_{s}(y):=\left\{\mathbb{J} \subset\{1,2, \ldots, n\}|| \mathbb{J}\left|=s, \min _{j \in \mathbb{J}}\right| x_{j}\left|\geq \max _{j \notin \mathbb{J}}\right| x_{j} \mid\right\} .
$$

Proof. (6.14)-(6.17) is a specification of [16, Example 3.14]. (6.18) can be found, for instance, in [76] or [122].

Except for the amplitude constraint and sparsity constraint all other presented constraint sets are closed and convex. To see that the amplitude constraint is nonconvex, take an element $x \in A$. Then $-x \in A$ as well. Assuming that $A$ is convex, the convex combination $\frac{1}{2} x+\frac{1}{2}(-x)=0$ has to be an element of $A$ too, which is a contradiction. This serves as an explanation why the sets $M_{j}$ are nonconvex as well. The sparsity constraint is nonconvex as well. It is known to be not prox-regular at all of its points. This and the type of regularity of the constraint sets is later discussed in Remark 6.3.3. 


\subsection{Regularities of Constraint Sets}

The phase retrieval algorithms we will analyze in Section 6.4 and Section 6.5 are projection methods. As discussed in Chapter 3 the regularities of sets have an impact on the regularity of the corresponding projector and thus on the projection method. Moreover, the regularity of the method influences the convergence behavior of the sequence generated by the projection method. This section is, therefore, devoted to set-regularities as in Section 3.3, but also to the regularities of sets involved in the phase retrieval problem as introduced in Section 6.1.

In the phase retrieval problem one type of nonconvexity, that is also covered by $\epsilon$-subregularity, is prox-regularity.

Definition 6.3.1 (prox-regular sets). A closed set $\Omega$ is called prox-regular at $\bar{x} \in \Omega$ if for $\bar{v} \in N_{\Omega}(\bar{x})$ there exist $\epsilon, \delta>0$ such that

$$
\frac{\epsilon}{2}\|x-c\|^{2} \geq\langle v, x-c\rangle \quad\left(\forall x, c \in \Omega \cap \mathbb{B}_{\delta}(\bar{x})\right)\left(\forall v \in N_{\Omega}(c) \cap \mathbb{B}_{\delta}(\bar{v})\right) .
$$

This definition dates back to Federer [62] who called the property sets with positive reach. The definition presented here is taken from [115, Proposition 1.2]. The authors in [115] showed that their definition of prox-regularity at $\bar{x} \in C$ is equivalent to several statements. One of the most prominent might be local singlevaluedness of the projector [115, Theorem 1.3] around $\bar{x}$. Kruger et al. showed that prox-regularity implies $\epsilon$-subregularity in [88, Proposition 4(vi)]. Moreover, by Example 3.3.2(ii) it is easy to see that every convex set is also prox-regular.

Lemma 6.3.2 (prox-regularity implies super-regularity at a distance). Let $\Omega$ be prox-regular at a point $\bar{x} \in \Omega$. Then $\Omega$ is super-regular at a distance at $\bar{x}$ relative to $\Lambda=\mathcal{E}$.

Proof. By [88, Proposition 4(vi)] $\Omega$ is super-regular at $\bar{x}$. Applying Remark 3.3.7, we deduce super regularity at a distance relative to $\Lambda=\mathcal{E}$.

Note that, as explained in Remark 3.3.7, we cover more than just super-regularity with the theory developed in Chapter 4 . We need super regularity at a distance to incorporate the fixed points that may not lie in any of the sets involved and achieve a desired averagedness of the mapping (see for instance Proposition 3.3.12 and Theorem 4.2.3). As the next remark shows, all constraint sets involved in the phase retrieval problem are, in fact, prox-regular, and thus super-regular at a distance by Lemma 6.3.2.

Remark 6.3.3 (phase retrieval constraint sets are prox-regular, hence super-regular at distance). Super-regularity at a distance of the measurement sets defined in (6.3),(6.4) and (6.5) is of great importance for the convergence analysis of the introduced algorithms. As noted in (6.6) all three sets are related by unitary mappings to each other. Thus, all three sets in fact describe coordinatewise circles 
of different radius in $\mathbb{R}^{2}$ when all other elements are fixed as noted before for $\mathcal{M}_{j}$. By Example 3.3.10 a circle $\Omega$ in $\mathbb{R}^{2}$ is super-regular at a distance relative to $P_{\Omega}^{-1}(\bar{x})$ at any of its points $\bar{x}$ and hence, (6.3),(6.4) and (6.5) are super-regular at a distance. As mentioned before $\epsilon$-subregularity covers a divers range of regularity notions for sets. The measurement sets investigated here are not only super-regular at a distance. In fact they are shown to be semi-algebraic [77, Proposition 3.5] and prox-regular by [115, Theorem 1.3] and the description of the projector given in Lemma 6.2.1.

The other sets that are involved in the phase retrieval problem are the qualitative constraints introduced in (6.7)-(6.11). Except for the amplitude constraint and the sparsity constraint all of these sets are convex and thus by Proposition 3.3.9 superregular at distance relative to $\mathcal{E}$. Fortunately, the amplitude constraint describes a circle in each coordinate like the measurement constraint. Hence, the amplitude constraint is super-regular at a distance as well (and additionally semi-algebraic and prox-regular). The sparsity constraint $\mathcal{A}_{s}$ is prox-regular at all points $\bar{x}$ satisfying $\|\bar{x}\|_{0}=s$. This can be seen by a similar argument like in the proof of $[122$, Proposition 4.4]).

\subsection{Phase Retrieval Algorithms}

Having a problem, one is seeking for a solution to solve it. Numerically we do this by using algorithms, and here, in particular, projection algorithms. Among these, the most prominent, and probably one of the easiest to compute, is the method of alternating projections. Given a point, it generates the next iterate by consecutively projecting onto each of the individual sets (see Algorithm 2.3.3 and Algorithm 2.3.2). Applying the alternating projection algorithm to the phase retrieval problem one set, $\mathcal{O}$, is identified with some of the additional constraint sets defined in (6.7)-(6.10). The other set represents one of the measurements $M_{j}$. If only one image is taken, i.e. $m=1$, one can define the famous error reduction algorithm via the method of alternating projections.

Algorithm 6.4.1 (error reduction). The error reduction algorithm produces the same sequence of iterates as the method of alternating projections on the sets $M_{1}$ and $\mathfrak{S}$. That is, given some $x^{0} \in \mathbb{C}^{n}$ generate the sequence $\left(x^{k}\right)_{k \in \mathbb{N}}$ by

$$
x^{k+1} \in P_{\mathfrak{S}} P_{M_{1}} x^{k} .
$$

The name of the algorithm goes back to Fienup [63-67], and preparatory work by Gerchberg and Saxton and their Gerchberg-Saxton algorithm [69]. The GerchbergSaxton algorithm was originally defined for retrieving the phase from two measurements. The connection of error reduction to alternating projections was made by Levi and Stark in [91]. Having again only a single measurement, we introduce the prominent hybrid input output method by Fienup [66]. 
Algorithm 6.4.2 (Fienup's hybrid input output method (HIO)). Given some point $x^{0} \in \mathbb{C}^{n}$ the $H I O$ algorithm is defined by

$$
x_{j}^{k+1} \in \begin{cases}P_{M_{1}} x^{k}{ }_{j} & \text { if } j \in I \\ x_{j}^{k}-P_{M_{1}} x^{k}{ }_{j} & \text { otherwise. }\end{cases}
$$

Rewriting this yields

$$
x^{k+1} \in\left(P_{\mathfrak{S}}\left(2 P_{M_{1}}-\mathrm{Id}\right)+\left(\mathrm{Id}-P_{M_{1}}\right)\right)\left(x^{k}\right),
$$

which is in fact the Douglas-Rachford algorithm, defined in Algorithm 2.3.4, applied to the sets $M_{1}$ and $\mathfrak{S}$.

The connection of HIO and Douglas-Rachford was observed by Bauschke et al. [16].

The main focus of the chapters above was on analyzing the relaxed DouglasRachford method. This algorithm enjoys great popularity in application to the phase retrieval problem (see for instance $[9,44,84,119]$ and many others).

Algorithm 6.4.3 (relaxed Douglas-Rachford applied to the phase retrieval problem). Given an initial point $x^{0} \in \mathcal{E}$ and a parameter $\lambda \in(0,1)$ the relaxed DouglasRachford method applied to the phase retrieval problem generates the sequence $\left(x^{k}\right)_{k \in \mathbb{N}}$ by

$$
x^{k+1} \in T_{\mathcal{O}, M_{1}}^{\lambda} x:=\bigcup_{b \in P_{M_{1}} x}\left\{\frac{\lambda}{2}\left(R_{\mathcal{O}}(2 b-x)+x\right)+(1-\lambda) b\right\} .
$$

This method, in the physics community, is also known by the term Relaxed Averaged Alternating Reflections, which was used by Luke in [97,98].

Remark 6.4.4. For the sake of completeness we want to mention other projection methods for solving the phase retrieval problem than the just introduced algorithms. These are, for example, the Hybrid Projection Reflection algorithm (HPR) [17], difference map [59], solvent flipping algorithm [1], and Fienup's Basic Input-Output algorithm (BIO) which is, in fact, nothing more than Dykstra's algorithm, see [16].

Remark 6.4.5. In addition to the formulation as a feasibility problem, one can also define the phase retrieval problem as an optimization problem as mentioned in Remark 2.3.7. To see this, we square the equation in (6.2) and deduce

$$
\left|\left(\mathcal{F} \cdot \mathcal{P}_{j} z\right)_{k}\right|^{2}=b_{k j}^{2}, \quad(\forall j=1,2, \ldots, m)(\forall k=1,2, \ldots, n) .
$$

A solution $z$ to these equations minimizes each of the following squared distances

$$
F_{j k} z:=\left\|\left|\left(\mathcal{F} \cdot \mathcal{P}_{j} z\right)_{k}\right|^{2}-b_{k j}^{2}\right\|^{2}, \quad(\forall j=1,2, \ldots, m)(\forall k=1,2, \ldots, n) .
$$


Thus, a solution $z$ to (6.2) is also a solution to the following minimization problem

$$
\operatorname{minimize}_{z \in C_{0}} F z:=\sum_{\substack{j \in\{1,2, \ldots, m\} \\ k \in\{1,2, \ldots, n\}}} F_{j k} z .
$$

The reformulation as an optimization problem in (6.22), where we minimize a function over some domain, is commonly addressed by forward-backward procedures. That is, given some initial point $x^{0}$ define the sequence $\left(x^{k}\right)_{k \in \mathbb{N}}$ via

$$
x^{k+1}=P_{M_{0}}\left(x^{k}-\lambda \nabla F\left(x^{k}\right)\right),
$$

where $\lambda$ is a nonnegative constant (see also Remark 2.3.7).

\subsection{Convergence Results}

In Chapter 4, we analyzed how to apply our basic convergence framework in Theorem 3.2.6 on the method of relaxed Douglas-Rachford. This section is devoted to the analysis of other algorithms, which we introduced in Section 6.4. While the analysis in Chapter 4 was detailed and focused on the assumptions of Theorem 3.2.6 in the context of set-feasibility, this section aims to present results to provide a broad intuition of the convergence of projection based algorithms used to solve the phase retrieval problem. This explains also, why the statements given next are presented in a cartoon like manner. Their statements include only the most important parts that yield local convergence. But not, how to construct it nor at which rate. Nevertheless, these are verifiable by following the approach by Luke, Tam and Thao in [105], which we used in Chapter 4 as a template for our convergence result.

Corollary 6.5.1 (convergence of the error reduction algorithm). Let $\bar{x}$ be a fixed point of the error reduction method. That is, $\bar{x} \in$ Fix $P_{\mathfrak{S}} P_{M_{1}}$. Then, the error reduction algorithm, defined in Algorithm 6.4.1, converges locally linearly to a point $\tilde{x} \in$ Fix $P_{\mathfrak{S}} P_{M_{1}}$ whenever the mapping $\Phi=P_{\mathfrak{S}} P_{M_{1}}$ - Id is locally metrically subregular at its zeros.

Proof. Following Burke, Luke and Lyon in [100, Section 3.2.2.], we represent $\mathbb{C}$ as $\mathbb{R}^{2}$ and reformulate the phase retrieval problem as a feasibility problem with entrywise values in $\mathbb{R}^{2}$. Then we can apply Theorem 3.2.6 using Remark 6.3.3, which concludes the proof.

A detailed analysis of the method of cyclic projections in the framework of Theorem 3.2.6 can be found in [105]. 
Remark 6.5.2. In contrast to Theorem 4.4.2 metric subregularity is required directly in Corollary 6.5.1. Equivalently, we could demand subtransversality of the collection of sets $\left\{\mathfrak{S}, M_{1}\right\}$ plus the additional assumption (ii) in Theorem 4.4.2. The problem here is that, up till now, it is not clear when and where these two assumptions are satisfied. Illustrative examples and numerical simulations indicate that they hold in lots of instances (see for instance Section 4.6). Nevertheless, there are certain situations when at least one of the two assumptions is violated. Moreover, allowing metric subregularity under some gauge, describes the reality sometimes better than restricting the analysis to a linear setting (see Definition 3.2.3). One example is the setting of alternating projections applied to the sphere $\mathbb{S}$ and a line tangent to $\mathbb{S}$ at $\bar{x}=(0,-1)$. In this instance the algorithm does not converge linearly to $\bar{x}$, although it converges depending on the initial point (see [105]). This problem is not only interesting for the type of convergence, but also when it comes to the actual numerical implementation of algorithms. Although sets in real-life applications almost never intersect tangentially, they do so because of numerical errors. In an instance where the sets are almost tangential to each other, they appear to be tangential in computation when the machine precision is too coarse in relation to being almost tangential. Thus, strong inconsistency and therefore measurement errors, that seem to be bad at first sight, can be actually an advantage for the numerical performance of an algorithm. We discuss this in more detail in Section 6.6.

Theorem 6.5.3 (convergence of Fienup's HIO method). Let $\bar{x}$ be a fixed point of Fienup's HIO method. That is, $\bar{x} \in \mathrm{Fix}\left(P_{\mathfrak{S}}\left(2 P_{M_{1}}-\mathrm{Id}\right)+\left(\mathrm{Id}-P_{M_{1}}\right)\right)$. Then, the HIO algorithm, defined in Algorithm 6.4.2 converges locally linearly to a point

$$
\tilde{x} \in \operatorname{Fix}\left(P_{\mathfrak{S}}\left(2 P_{M_{1}}-\mathrm{Id}\right)+\left(\mathrm{Id}-P_{M_{1}}\right)\right)
$$

whenever the mapping $\Phi=\left(P_{\mathfrak{S}}\left(2 P_{M_{1}}-\mathrm{Id}\right)+\left(\mathrm{Id}-P_{M_{1}}\right)\right)-\mathrm{Id}$ is locally metrically subregular at its zeros.

Proof. Since Fienup's HIO can be identified with the Douglas-Rachford method the result follows from [105, Theorem 3.4], using the same representation of $\mathbb{C}$ as $\mathbb{R}^{2}$ as mentioned in the proof of Corollary 6.5.1.

Noisy measurements make the phase retrieval problem become almost always an inconsistent feasibility problem. Hence, it is not likely that

$$
\Phi=\left(P_{\mathfrak{S}}\left(2 P_{M_{1}}-\mathrm{Id}\right)+\left(\mathrm{Id}-P_{M_{1}}\right)\right)-\mathrm{Id}
$$

is metrically subregular at its zeros. Instead of the HIO, one can use a relaxed version of Douglas-Rachford that we introduced in Algorithm 2.3.5 to adapt to the inconsistent setting.

Theorem 6.5.4 (convergence of relaxed Douglas-Rachford applied to the phase retrieval problem). Let $\bar{x} \in$ Fix $T_{\mathcal{O}, M_{1}}^{\lambda}$ for $T_{\mathcal{O}, M_{1}}^{\lambda}$ defined in Algorithm 2.3.5. The 
relaxed Douglas-Rachford applied to a phase retrieval problem converges locally linearly to a point $\tilde{x} \in$ Fix $T_{\mathcal{O}, M_{1}}^{\lambda}$ whenever the mapping $\Phi=\frac{\lambda}{2}\left(R_{\mathcal{O}} R_{M_{1}}+\mathrm{Id}\right)+(1-$ ג) $P_{M_{1}}$ - Id is locally metrically subregular at its zeros.

A detailed convergence analysis for the relaxed Douglas-Rachford algorithm was carried out in Chapter 4. In contrast to the original Douglas-Rachford method, the big advantage of its relaxed version is that the set of fixed points does not have to be empty in an inconsistent setting. Which is in fact the case for the Douglas-Rachford method, see for instance [18, Theorem 3.13]. This indicates that the relaxed Douglas-Rachford method is somehow more stable with respect to inconsistency. Connecting this observation to the convergence analysis presented here, we claim that in practice the Douglas-Rachford method has less chances to work than its relaxed version in regard to consistency. This becomes more clear when considering the function $\Phi=T$-Id that has to be locally metrically regular at its zeros. But the zeros of $\Phi$ are nothing more than the fixed points of the chosen method $T$. Thus, if no fixed points exist, the method has no chance to satisfy this assumption of Theorem 3.2.6 and therefore to converge. When considering the fixed point set of relaxed Douglas-Rachford itself, there is another benefit of the procedure. The fixed points of the method do not have to be points in the intersection as seen in Theorem 4.2.3. Varying the parameter $\lambda$, we can wander as far away from the intersection, or local gap, as we want. Thus, chances are low that we get stuck in a local minimum as it can happen with alternating projections. Although we did not give detailed proofs for our convergence statements, we refer the reader to Section 4.6 for an intuition of the assumptions and when to expect them to be satisfied. The examples considered there were circles representing the entries of a vector satisfying the measurement constraint $M_{1}$.

Other algorithms that we mentioned were the cyclic relaxed Douglas-Rachford method, and Fienup's BIO algorithm. Following the ideas made before, it is not hard to show that both methods are defined by almost averaged mappings when applied to the phase retrieval problem. Nevertheless, understanding the property of metric subregularity in the context of feasibility problems fully is still an open problem. Thus, local convergence on its own can be easily verified but it is hard to quantify it.

\subsection{Numerical AnAlysis}

In addition to the theoretical investigation of an algorithm it is worth discussing its actual numerical performance when it comes to real-life applications. Therefore, we devote this section to the numerical analysis of the introduced projection methods with a particular focus on the relaxed Douglas-Rachford method. All experiments carried out below were created with Matlab using data sets provided by the ProxToolbox of Luke and coauthors, which can be found online via http://num.math.uni-goettingen.de/proxtoolbox/. 
We already noticed in Remark 6.5.2 that the rate of convergence, or convergence at all, depends on the numerical precision in computation. But also inconsistency has an impact on the numerical performance. Closely related to this, we want to stress another feature of the presented analysis. That is, less information can improve the performance of your algorithm. For a demonstration we analyze a data set called "tasse" recorded by undergraduates at the X-Ray Physics Institute at the University of Göttingen. It is an optical diffraction image with model constraints $b_{i 1}, \quad i=1,2, \ldots, n$, as in (6.3) with $m=1$ and $n$ the dimension of the image describing far field data and an additional support constraint (see Fig. 6.1).

The full data set has dimension $n=1392 \times 1040$, the limited, or cropped, data set $n=128^{2}$. The following graphs are created by applying the relaxed DouglasRachford method to the problem instance for both the full data set and the limited data set. In both instances we used a dynamic relaxation parameter $\lambda$, generated by a smoothed step function that evolves from 0.95 to 0.5 after 30 iterations as suggested by Luke in [97]. The relaxed Douglas-Rachford method applied to the full data set shows a worse convergence behavior than the relaxed Douglas-Rachford method applied to the limited data set (see Fig. 6.2). Not only the algorithm needs more iterations to reach a certain accuracy $\left(9.0147 \times 10^{4}\right.$ instead of 947$)$, but also the rate of linear convergence, when the iterates reach a suitable neighborhood, is worse. As shown in Fig. 6.3 the relaxed Douglas-Rachford method applied to the full data set needs more than $5 \times 10^{4}$ iterations to show a linear slope. Noteworthy, is the observed gap in both problem instances. In the full data set version the gap is smaller than in the version with a limited data set (see Fig. 6.4). We conjecture that this observed behavior is due to the property of metric subregularity, or in the context of set feasibility, subtransversality. The more and better information one has, the closer the constraint sets come to each other. Thus, they are more likely to intersect. This also includes cases, in which the sets intersect transversally as well. In these cases, the relaxed Douglas-Rachford method does not have to converge
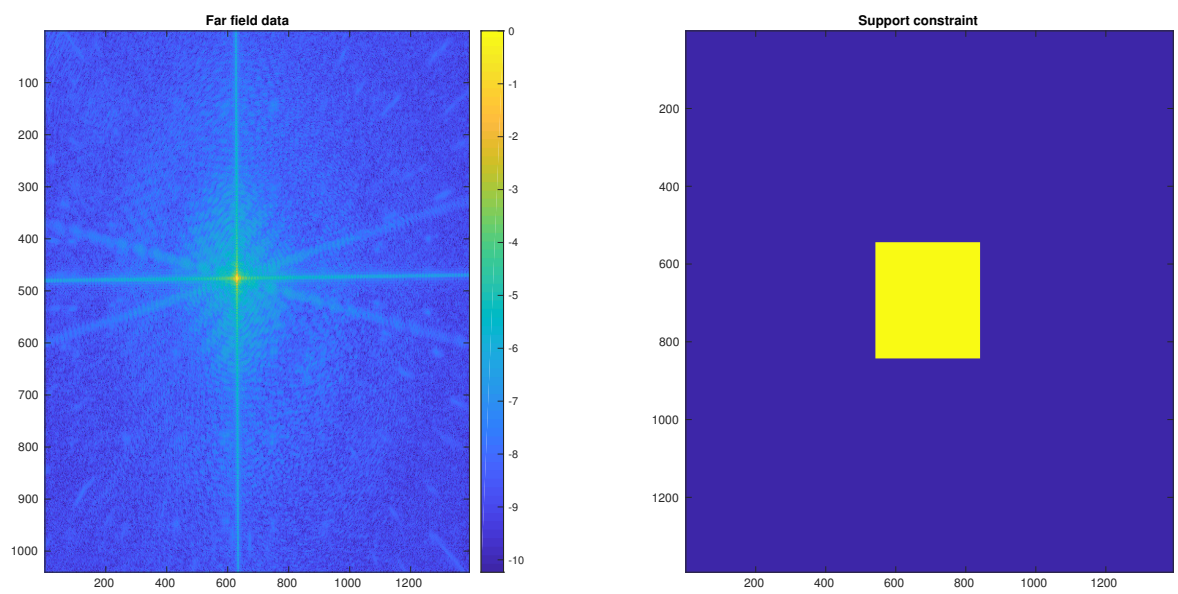

Figure 6.1: Measurement and support constraint. 


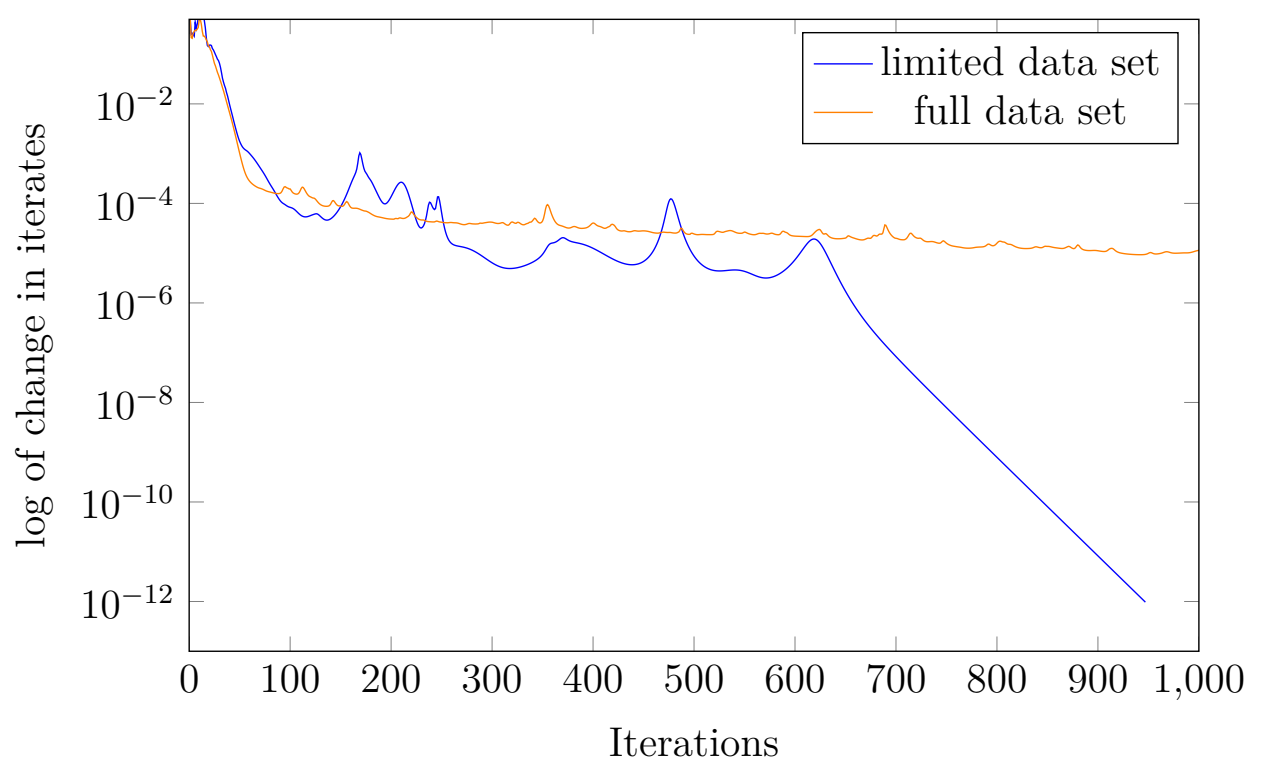

Figure 6.2: Change in iterates for data set "tasse" until iteration 1000.

locally linear but can show sublinear convergence (see for instance [105, Remark 3.2] for the method of alternating projections). The take home message in this context is that more information does not have to yield a better image, when applying numerical algorithms. Note that these results are not limited to the method of relaxed Douglas-Rachford. We observed similar results with alternating projections applied to the same data set.

In Chapter 4 we discussed the performance of the relaxed Douglas-Rachford method and compared it to other algorithms in the context of subspaces (see Section 4.7).

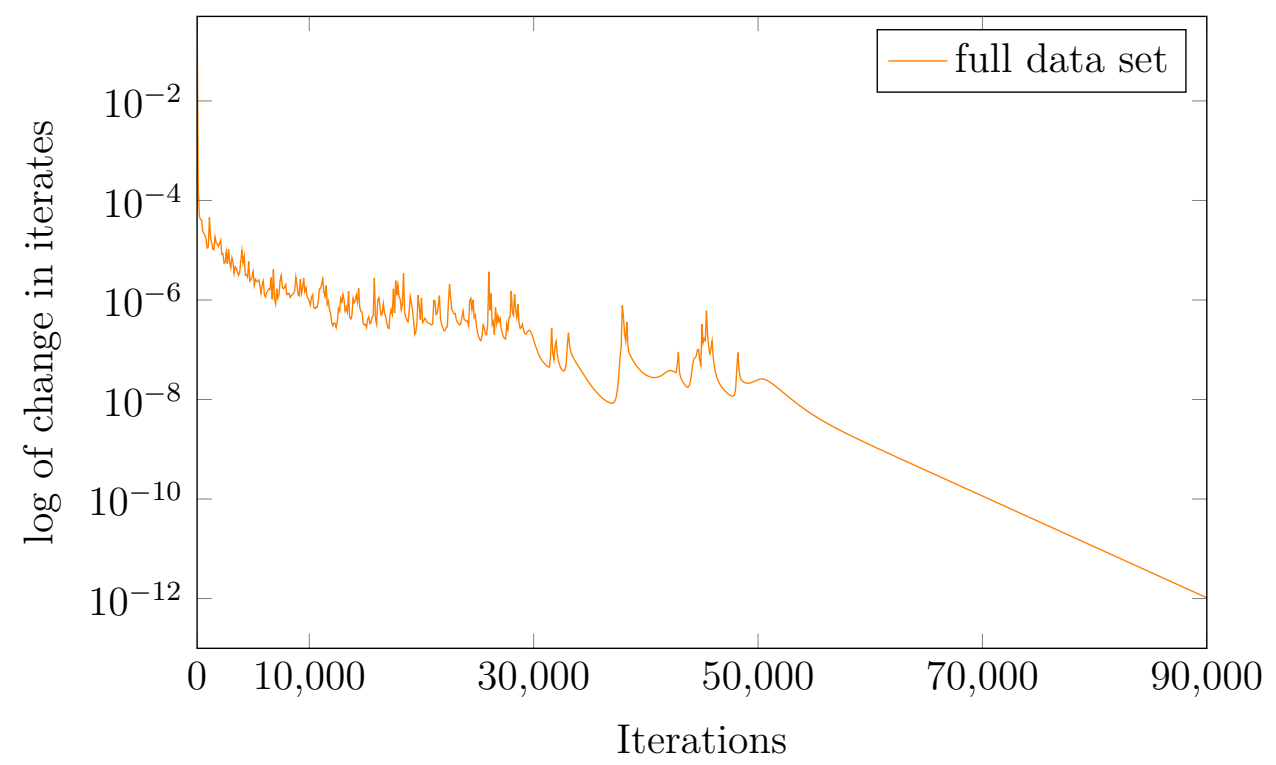

Figure 6.3: Change in iterates for full data set "tasse". 


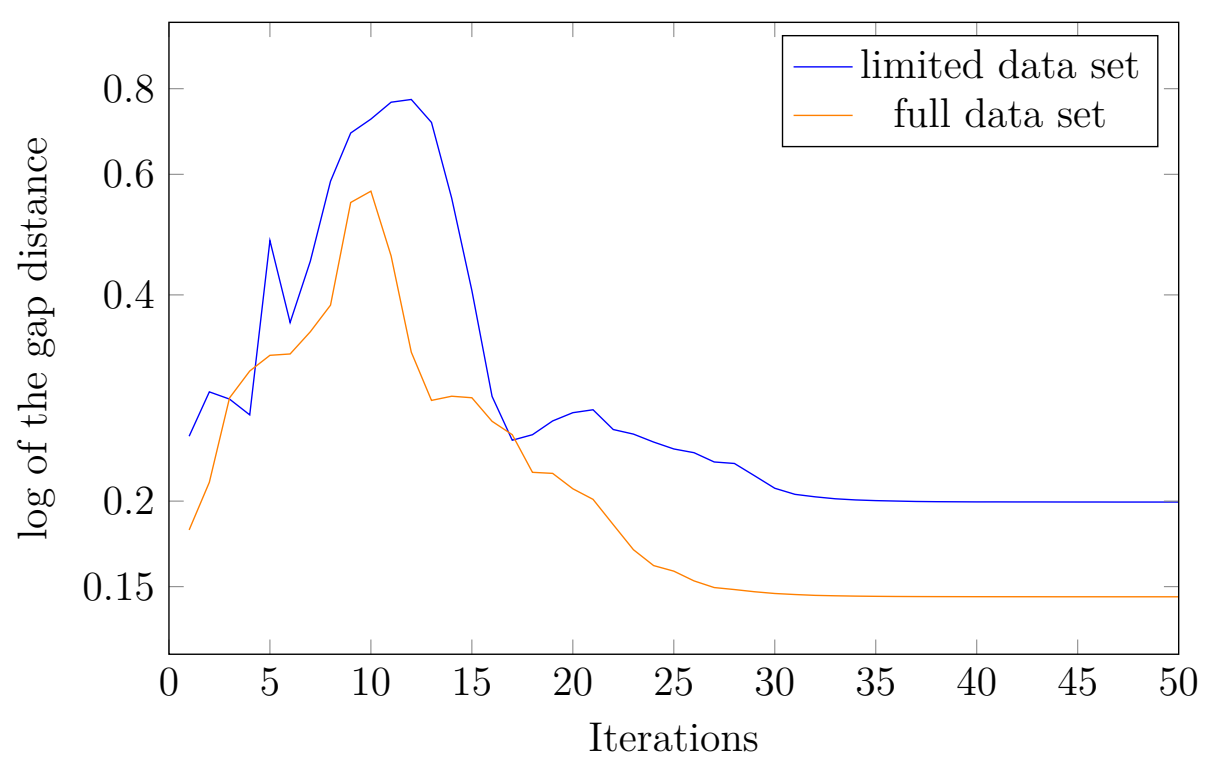

Figure 6.4: Gap for data set "tasse" until iteration 50.

In this convex regime the convergence rates we calculated were not promising when compared to standard procedures, like the method of alternating projections or the Douglas-Rachford method. The aim of this paragraph is to show the strength of relaxed Douglas-Rachford and its cyclic version in nonconvex feasibility instances. We use again the data set "tasse" introduced before in the size $n=128^{2}$. Note that the strength of this data set is that it is experimental data not artificially constructed data. We applied alternating projections to it as well as cyclic relaxed Douglas-Rachford $(\lambda=0.9)$ and the relaxed Douglas-Rachford method with a fixed relaxation parameter $\lambda=0.5$ and a dynamic parameter. As before, the relaxation parameter is generated by a smoothed step function that evolves from 0.95 to 0.5 after 30 iterations. The log of the change in the iterates for one randomly generated, but for all algorithms the same, instance are displayed in Fig. 6.5. We observe that in this particular instance the cyclic relaxed Douglas-Rachford method reached the stopping criterium (log of change in iterates is less or equal than $10^{-12}$ or the maximum number of iterations $\left(10^{5}\right)$ is exceeded) the fastest (see also Table 6.1). Note that all four algorithms show a linear slope after some iterations. This is in line with the results in this thesis and the one by Luke, Thao and Tam in [105]. All four algorithms find the same gap of roughly 0.2 as illustrated in Fig. 6.6. While relaxed Douglas-Rachford with a dynamic parameter needs roughly 35 iterations till the iterations attain the gap of 0.2 , the other algorithms reach it sooner. We conjecture that this has to do with the dynamical change of the parameter after 30 iterations. Luke conjectured that, due to the dynamic relaxation, a suitable neighborhood of convergence might be reached faster. Nevertheless, this algorithm was by far the slowest algorithm of the four used in this example. Even though we have to emphasize that this is just one example. Therefore, we extended this experiment to 100 randomly generated instances to better compare the four algorithms. The average number of iterations till the stopping criterium is reached as well as the 


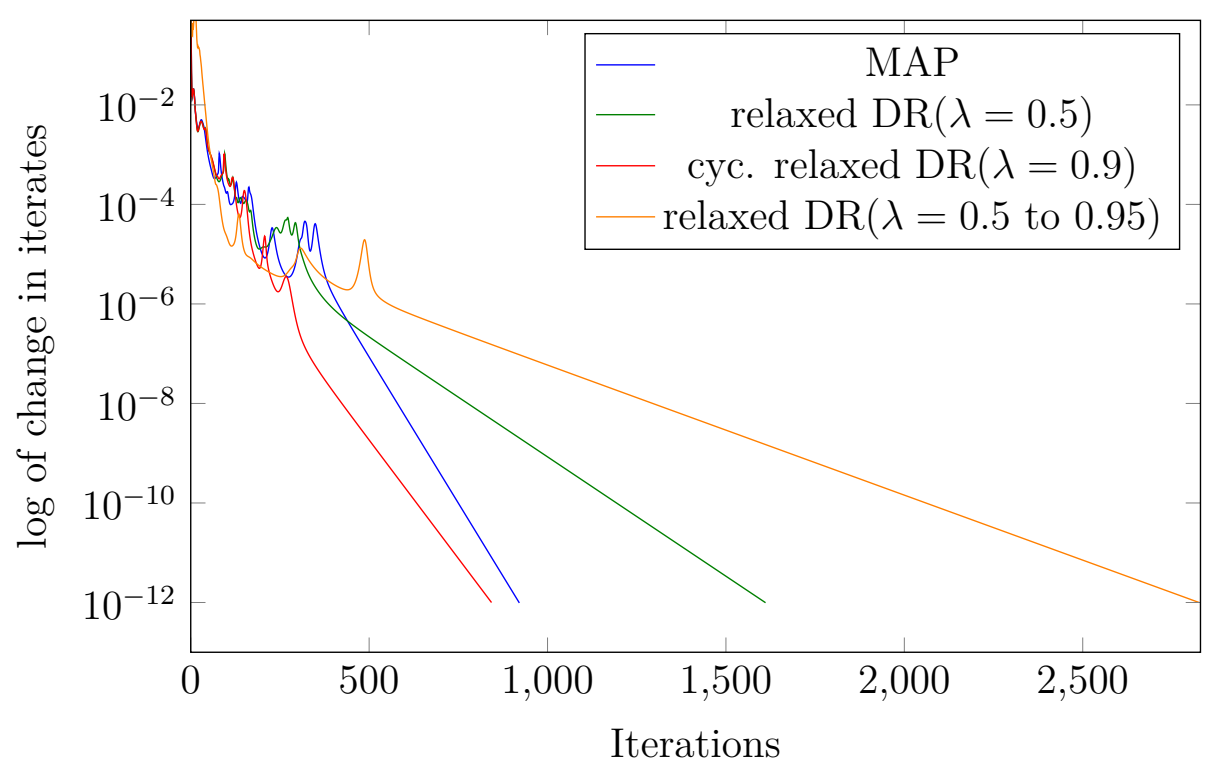

Figure 6.5: Change in iterates for limited data set ("tasse").

minimal amount and maximal amount of iterations needed are shown in Table 6.2. It becomes apparent that the four algorithms roughly show need the same number of iterations. Nevertheless, the relaxed Douglas-Rachford method with dynamic parameter shows the best performance. It needed the smallest number of iterations on average, but also generated sequences whose maximum and minimum number of iterations is less than the numbers of the other algorithms. This observation supports Luke conjecture on the behavior of the relaxed Douglas-Rachford method with dynamic relaxation parameter. There was no instance, under the 100 randomly generated, were one of the algorithms did not converge.

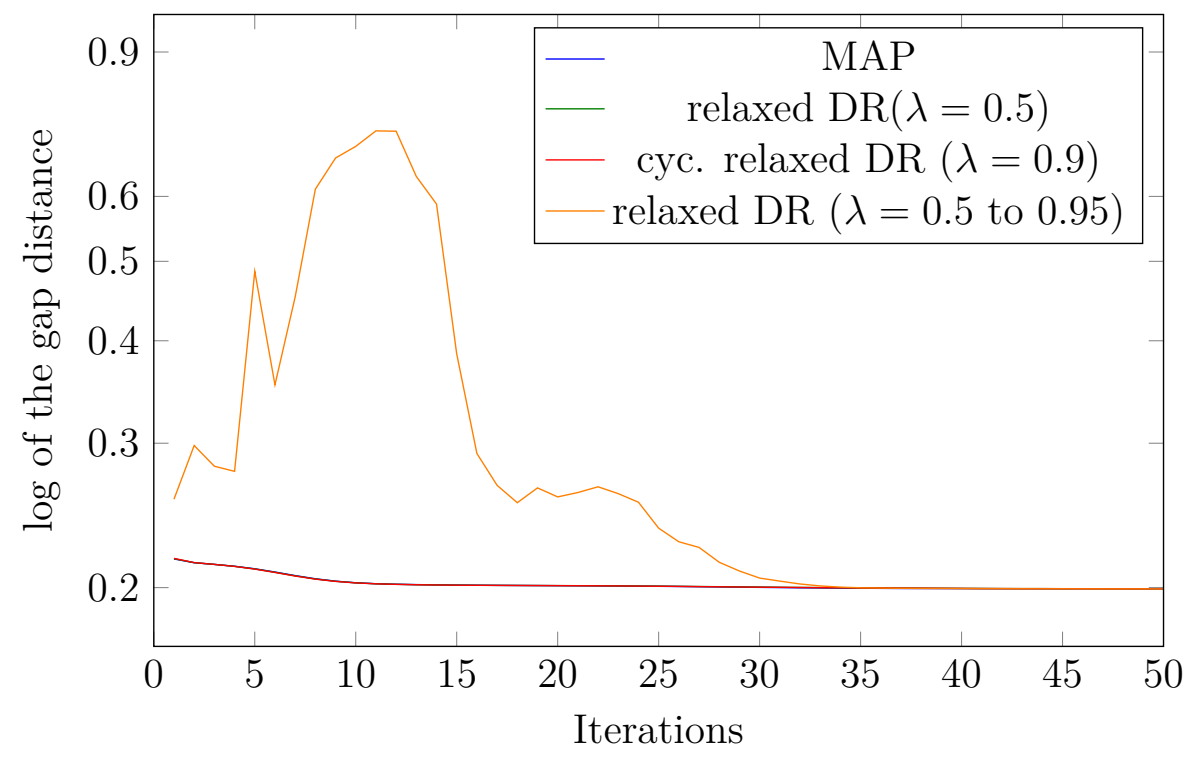

Figure 6.6: Gap for limited data set ("tasse") until iteration 50. 


\begin{tabular}{l|l}
\hline algorithm & iteration counts \\
\hline \hline cyc. relaxed $\operatorname{DR}(\lambda=0.9)$ & 843 \\
MAP & 921 \\
relaxed $\operatorname{DR}(\lambda=0.5)$ & 1611 \\
relaxed $\operatorname{DR}(\lambda=0.5$ to 0.95$)$ & 2826
\end{tabular}

Table 6.1: Comparison of the number of iterations till stopping criterium is reached of MAP, relaxed DR and its cyclic version.

We observed that the relaxed Douglas-Rachford algorithm and its cyclic version in our experimental setup were unstable with respect to the relaxation parameter. The choice of the relaxation parameter for the relaxed Douglas-Rachford method and its cyclic version present rather good results. When varying the relaxation parameter a bit, the results can look way worse. Further investigation of this behavior is needed.

Although we are able to observe if the iterates of the algorithms used do converge or not, we cannot say if the reconstructions made are "good" from the behavior of the iterates. The reconstructions in Fig. 6.7 look very similar to each other for the human eye. Nevertheless, the focus of this thesis was not on an error analysis of the relaxed Douglas-Rachford method. Therefore, we omit it here and refer the reader to the following two studies on the discussed projection methods: in [97] Luke compared the relaxed Douglas-Rachford method to HIO and HPR using an error measure going back to Fineup [66]; in [17] Bauschke, Combettes and Luke compared the HIO and HPR on noisy and noisless data using the same measure.

To compare the algorithms accuracy besides their iteration count, we focus next on a synthetic data set. In fact, we consider the James Webb Space Telescope wavefront reconstruction data set first used in [100] by Burke, Luke and Lyon. This data set was constructed to simulate the actual telescope that has yet not been installed. The dimension of the image is $n=128 \times 128$. Given are two out-of-focus images and one in-focus images $(m=3)$ and an additional amplitude constraint, which sets the amplitude of the wavefront to be constructed to one across the aperture of the telescope (see [100]). Like before, we run the different algorithms 100 times on

\begin{tabular}{l|l|l|l}
\hline & \multicolumn{3}{|c}{ iteration counts } \\
algorithm & mean & maximum & minimum \\
\hline \hline relaxed DR $(\lambda=0.5$ to 0.95) & 990 & 5004 & 449 \\
cyc. relaxed DR $(\lambda=0.9)$ & 1215 & 5352 & 509 \\
MAP & 1274 & 6142 & 507 \\
relaxed DR $(\lambda=0.5)$ & 1306 & 6854 & 585
\end{tabular}

Table 6.2: Mean, minimum, and maximum number of iterations of MAP, relaxed DR and its cyclic version for 100 random instances. 


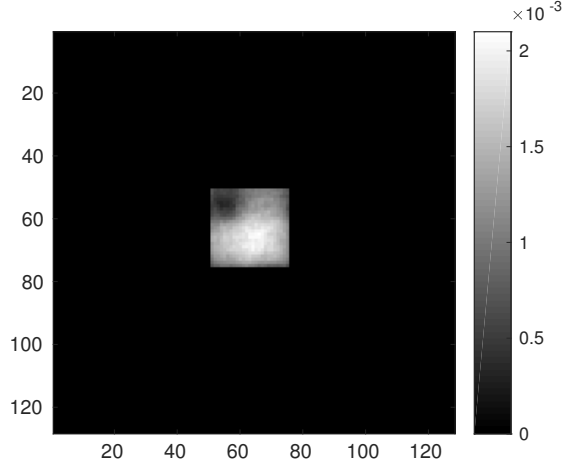

(a) MAP

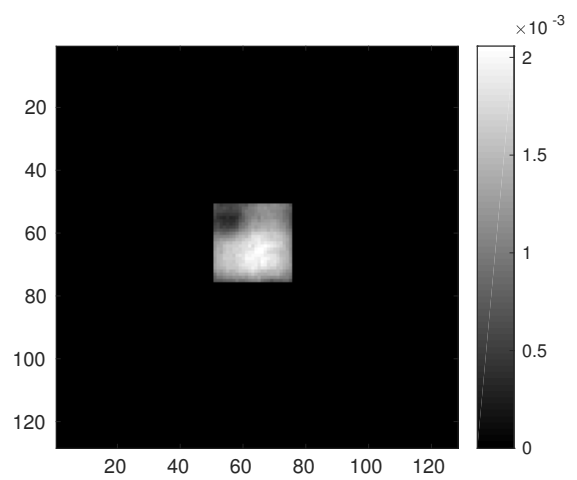

(c) relaxed DR $(\lambda=0.5$ to 0.95$)$

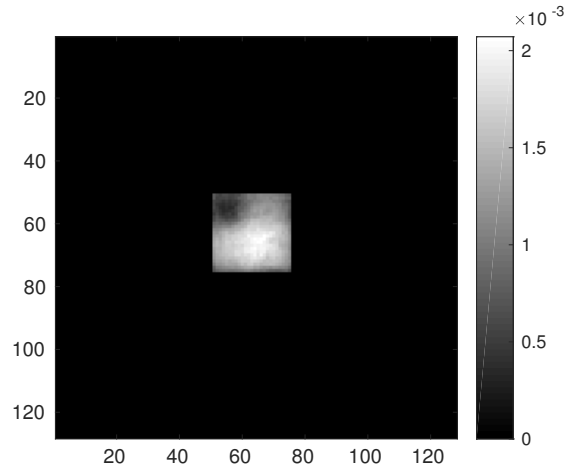

(b) relaxed DR $(\lambda=0.5)$

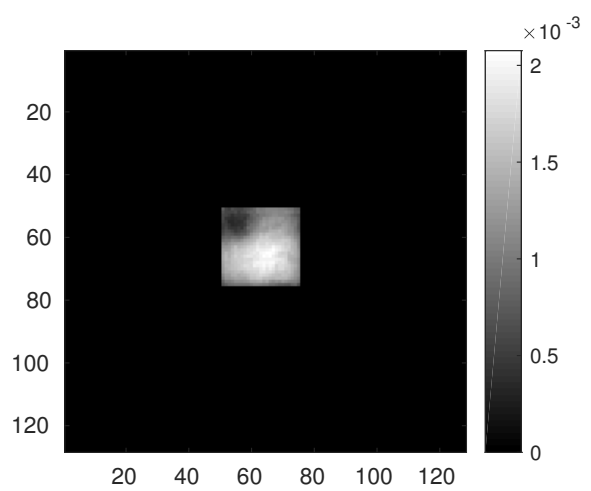

(d) cyclic relaxed DR

Figure 6.7: Reconstruction of the optical diffraction experiment "tasse" using MAP, relaxed DR and its cyclic version.

randomly chosen initial points. The stopping criteria is reached once the log of the difference between successive iterates falls below $5 \times 10^{-5}$, or a maximum iteration count of 6,000 is reached. We decided for relaxed Douglas-Rachford to switch from $\lambda=0.5$ to $\lambda=0.9$ dynamically after 20 iterations. Table 6.3 presents the results concerning the number of iterations. The smallest mean shows the cyclic relaxed Douglas-Rachford instances with 24 iterations. While cyclic projections produced an instance with the smallest amount of iterations with 17 iterations. This is close to the minimum iteration count of cyclic relaxed Douglas-Rachford (20 iterations). Overall the table shows that over 100 random instances cyclic relaxed DouglasRachford outperforms the other three algorithms. Again, the choice of $\lambda$ had a great impact on the results. Taking into account the relative error to the true solution, as shown in Table 6.4, the cyclic relaxed Douglas-Rachford outperforms the other three algorithms by far. Noteworthy is that the solution the iterates converge to, independent of the algorithm, is not a specific point, but to a fixed amplitude and fixed relative phase with a constant global phase shift from one iterate to the 


\begin{tabular}{l|l|l|l}
\hline & \multicolumn{3}{|c}{ iteration counts } \\
algorithm & mean & maximum & minimum \\
\hline \hline relaxed DR $(\lambda=0.5$ to 0.9$)$ & 93 & 133 & 75 \\
cyc. relaxed DR $(\lambda=0.9)$ & 24 & 43 & 20 \\
CP & 37 & 172 & 17 \\
relaxed DR $(\lambda=0.9)$ & 81 & 161 & 69
\end{tabular}

Table 6.3: Mean, minimum, and maximum number of iterations of CP, relaxed DR and its cyclic version for 100 random instances on synthetic data.

\begin{tabular}{l|l|l|l}
\hline & \multicolumn{3}{|c}{ error to true solution } \\
algorithm & mean & maximum & minimum \\
\hline \hline relaxed DR $(\lambda=0.5$ to 0.9) & 1.0651 & 1.1547 & 0.96205 \\
cyc. relaxed DR $(\lambda=0.9)$ & $1.5204 \times 10^{-} 6$ & $5.7247 \times 10^{-} 6$ & $1.1238 \times 10^{-} 7$ \\
CP & 3.4675 & 5.1304 & 2.0157 \\
relaxed DR $(\lambda=0.9)$ & 1.0580 & 1.2132 & 0.94077
\end{tabular}

Table 6.4: Mean, minimum, and maximum relative error to the true solution $\times 10^{-} 5$ of $\mathrm{CP}$, relaxed $\mathrm{DR}$ and its cyclic version for 100 random instances on synthetic data.

next as mentioned in [103]. We refer the reader for more experiments to [103], where the authors extensively compare the discussed algorithms.

We have seen that relaxed Douglas-Rachford does not have to perform better in "easy" setups, such as intersecting subspaces, or more sophisticated setups like the optical diffraction example in this section. Nevertheless, the relaxed DouglasRachford algorithm can be faster than other projection methods when the relaxation parameter is chosen wisely. Thus, it can outperform other algorithms in nonconvex inconsistent feasibility instances. While these observations might be not new (see for instance $[97,103]$ ), it emphasizes the importance of the theoretical analysis we gave in Chapter 4. Moreover, it highlights the need for an analysis of the relaxation parameter for the relaxed Douglas-Rachford algorithm and its cyclic version. 



\section{CHAPTER 7}

\section{A Matrix World Approach}

The theory presented in Chapter 4 and Chapter 5 focused on local convergence results to fixed points of the introduced algorithms. If the sets are nonconvex these fixed points that are approximations to the phase retrieval problem do not have to be solutions to the problem. Therefore, this chapter is devoted to the question of whether fixed points of algorithms can be unique solutions. By examining different approaches from the literature, we present assumptions that imply existence of a (up to a global phase) unique solution of the phase retrieval problem.

Focusing on intensity measurements only, Candès, Li and Soltanolkotabi introduced the idea of the phase lift for the phase retrieval problem in [39]. Instead of solving a nonconvex problem in $\mathbb{C}^{n}$, they reformulated the phase retrieval problem as a rank constrained affine problem in $\mathbb{C}^{n \times n}$. Acknowledging the fact that this "new" problem is NP hard [108], Candès and several coauthors came up with relaxation ideas for the problem (see for instance $[36,38,39]$ ). Their approach involves relaxing the phase retrieval problem to a trace-norm minimization problem and benefits from semidefinite programming. Even though the phase retrieval problem is ill-posed when including only measurement constraints, they decided to take this formulation and propose using different diffraction patterns according to a certain rule. In combination with their phase lift approach, they were able to show that their path can recover the object exactly (up to a global phase factor) with high probability if the number of measurements is of order $n \log (n)$ [39]. They refined their results in [36], showing that 3 measurements can be enough to achieve perfect recovery of the phase. Even though these results are impressive, the actual application of the algorithm is not reasonable because of the computational drawbacks; lifting the phase retrieval problem from a space of dimension $n$ to a space of dimension $n^{2}$ increases the time one needs for computation.

The route, we follow here is inspired by the approach of Candès and his coauthors, but different. Instead of relaxing the problem, which is, as we think, not necessary to achieve global results, we employ a restricted isometry property (RIP) (see Definition 7.0.1) assumption to state results on solutions to the phase retrieval problem. Moreover, our results are of a theoretical nature in the lifted space. For 
applications, we leave the problem, in contrast to Candès, in the original space $\mathbb{C}^{n}$ and thus we do not suffer from any computational drawbacks. We begin by lifting our problem from the $n$-dimensional space $\mathbb{C}^{n}$ to the matrix space $\mathbb{C}^{n \times n}$ of dimension $n^{2}$. For this we remind the reader on the phase retrieval problem with only measurement constraints, which are given by

$$
M_{j}:=\left\{z \in \mathbb{C}^{n}||\left(\mathcal{F} \cdot \mathcal{P}_{j} z\right)_{k} \mid=b_{k j}, \forall k=1,2, \ldots, n\right\},
$$

for $j=1,2, \ldots, m$. Then, the phase retrieval problem is given by

$$
\text { find } x \in \cap_{j=1}^{m} M_{j} \text {. }
$$

For simplicity, we denote $\mathcal{F} \cdot \mathcal{P}_{j}$ by $A_{j}$ and define $\mathcal{A}_{j k}:=\left(A_{j}\right)_{k}\left(A_{j}\right)_{k}^{*}$ for all $j=$ $1,2, \ldots, m$. To express $(7.2)$ in $\mathbb{C}^{n \times n}$, we rewrite (7.1) as follows.

$$
\begin{aligned}
\left|\left(\mathcal{F} \cdot \mathcal{P}_{j} z\right)_{k}\right|^{2} & =\left|\left(A_{j} z\right)_{k}\right|^{2} \\
& =\left|\left\langle\left(A_{j}\right)_{k}, z\right\rangle\right|^{2} \\
& =\operatorname{Tr}\left(z^{*}\left(A_{j}\right)_{k}\left(A_{j}\right)_{k}^{*} y\right) \\
& =\operatorname{Tr}\left(\left(A_{j}\right)_{k}\left(A_{j}\right)_{k}^{*} z z^{*}\right) \\
& =\operatorname{Tr}\left(\mathcal{A}_{j k} z z^{*}\right) \\
& =\operatorname{Tr}\left(\mathcal{A}_{j k} Z\right),
\end{aligned}
$$

where $Z:=z z^{*}$ and $\operatorname{Tr}(X)$ denotes the trace of the matrix $X \in \mathbb{C}^{n \times n}$. Note that $z z^{*}$ is a symmetric rank-one matrix. The mapping $\operatorname{Tr}\left(\mathcal{A}_{j k} \cdot\right)$ is linear and the sets

$$
\mathcal{M}_{j}:=\left\{X \in \mathbb{C}^{n \times n} \mid \operatorname{Tr}\left(\mathcal{A}_{j k} X\right)=b_{k j}, \forall k=1,2, \ldots, n\right\},
$$

for $j=1,2, \ldots, m$ are affine subspaces. With these observations we formulate the following feasibility problem

$$
\text { find } X \in S_{+}(n, 1) \cap\left(\bigcap_{j=1}^{m} \mathcal{M}_{j}\right) \text {, }
$$

where $S_{+}(n, 1)$ is the set of symmetric rank-one matrices. That is,

$$
S_{+}(n, 1):=\left\{X \in \mathbb{C}^{n \times n} \mid X=x x^{*} \text { for some } x \in \mathbb{C}^{n}\right\} .
$$

Now, a solution $z$ to (7.2) defines a solution to the problem (7.3) by $z z^{*}$. Beyond that, a solution $Z$ to (7.3) can be always represented by $z z^{*}$ for some $z \in \mathbb{C}^{n}$. The vector $z$ is a solution to (7.2) up to a global phase since $(z \exp (i \phi))(z \exp (i \phi))^{*}=$ $(z \exp (i \phi)) z^{*} \exp (-i \phi)=z z^{*}$ for any phase $\phi \in[0,2 \pi]$. Altogether, we can say that the feasibility problem (7.2) in $\mathbb{C}^{n}$ and (7.3) in $\mathbb{C}^{n \times n}$ are equivalent up to a global phase. Hence, we continue studying the feasibility problem (7.3).

(7.3) is a problem of finding a matrix in essentially two different types of sets: a rank constraint and an affine constraint. This problem class is closely related 
to sparse affine feasibility which asks for a point in the intersection of an affine constraint and a sparsity constraint. For more on the similarity of both problems, see for instance [122]. Although there is a rich literature which studies relaxations of this problem (see for instance $[31,41,89]$ ), we concentrate on uniqueness results for the feasibility problem, rather than on new algorithms. In [76] Luke, Hesse and Neumann studied the method of alternating projections and Douglas-Rachford applied to sparse affine feasibility. In addition to local convergence results, they were able to state a global convergence result for alternating projections under a restricted isometry property. The restricted isometry property was introduced by Candès and Tao [40] for sparse real-valued vectors. In this work, we use the following version for complex valued matrices defined as follows.

Definition 7.0.1 (restricted isometry property on $\mathbb{C}^{n \times n}$ ). Let $M$ be a matrix in $\mathbb{C}^{m \times(n \times n)}$ of full rank $\left(m \leq n^{2}\right)$. That is, $M$ is a linear mapping $M: \mathbb{C}^{n \times n} \rightarrow \mathbb{C}^{m}$. Then $M$ is said to satisfy the restricted isometry property of order $s$ if there exists $\delta \in[0,1]$ such that

$$
(1-\delta)\|X\|^{2} \leq\left\|M^{\dagger} M X\right\|^{2} \leq\|X\|^{2} \quad \forall X \in \mathbb{C}^{n \times n} \text { with } \operatorname{rank}(X) \leq s,
$$

where $M^{\dagger}$ denotes the Moore-Penrose inverse $M^{*}\left(M M^{*}\right)^{-1}$. The infimum over all such constants $\delta$ is called the restricted isometry constant.

The first generalization of Candès and Tao's RIP for the vector case to the matrix case dates back to Recht, Fazel and Parillo [117, Definition 3.1]. In contrast to our version, their matrices are real-valued. Moreover, the restricted isometry property used here is not scaled, which some authors favor. Scaled restricted isometry properties were for instance used in [27] or [76].

Next is our main result of this chapter. Following a result by Luke, Hesse and Neumann in [76] and a generalization of their work in Neumann's PhD thesis [109], we prove convergence of alternating projections between the two sets $S_{+}(n, 1)$ and $\left(\cap_{j=1}^{m} \mathcal{M}_{j}\right)$ as specified in (7.3). This result does not only include convergence but also uniqueness of the intersection of both sets. This part of the statement is much more interesting for our purposes, since our goal is to analyze when fixed points of algorithms are solutions to the phase retrieval problem and not just approximations.

Theorem 7.0.2 (global linear convergence of alternating projections in lifted space). Denote by $M$ the affine mapping describing the affine set $\left(\cap_{j=1}^{m} \mathcal{M}_{j}\right)$. That is,

$$
\bigcap_{j=1}^{m} \mathcal{M}_{j}=\left\{X \in \mathbb{C}^{n \times n} \mid M X=b\right\}
$$

for some suitable $b$. If $M$ satisfies the RIP of order 2 with $\delta \in[0,1 / 2)$, then $S_{+}(n, 1) \cap\left(\bigcap_{j=1}^{m} \mathcal{M}_{j}\right)$ is a singleton. Moreover, for any initial value $X^{0} \in S:=$ 
$\left\{X \in \mathbb{C}^{n \times n} \mid X^{*}=X\right\}$, the sequence $\left(X^{k}\right)_{k \in \mathbb{N}}$ generated by alternating projections on the two sets $S_{+}(n, 1)$ and $\bigcap_{j=1}^{m} \mathcal{M}_{j}$ converges to $S_{+}(n, 1) \cap\left(\bigcap_{j=1}^{m} \mathcal{M}_{j}\right)$ with dist $_{\cap_{j=1}^{m} \mathcal{M}_{j}}\left(X^{k}\right) \rightarrow 0$ as $k \rightarrow \infty$ at a linear rate with constant bounded above by $\sqrt{\frac{\delta}{1-\delta}}$.

Proof. Our proof is based on [76, Theorem III.15] of Luke, Hesse and Neumann and the generalization by Neumann in [109, Theorem 5.2.3.] between an affine set and the lower level set of a lower semicontinuous and subadditive function $\phi$ using a generalization of the RIP including $\phi$. Whereas Neumann proved his result for $\mathbb{R}^{n}$, we show the result for Hermitian matrices in $\mathbb{C}^{n \times n}$. We restrict the analysis made here to the rank function of a matrix. Nevertheless, following Neumann's approach a generalization to lower semicontinous and subadditive functions $\phi: \mathbb{C}^{n \times n} \rightarrow \mathbb{R}$ is possible.

We start by showing that $S_{+}(n, 1) \cap\left(\bigcap_{j=1}^{m} \mathcal{M}_{j}\right)$ is a singleton. Assume there exist $X, Y \in S_{+}(n, 1) \cap\left(\bigcap_{j=1}^{m} \mathcal{M}_{j}\right)$ with $X \neq Y$. Then, we have

$$
M X=M Y=b,
$$

where $b$ describes the constants $b_{k j}$ in relation to $M$. Since both $X$ and $Y$ are of rank one, their difference $X-Y$ is of rank at most two. Moreover, by the linearity of $\mathcal{A}_{j}$ and thus $M$ we have $M(X-Y)=M X-M Y=b-b=0$. This implies $\left\|M^{\dagger} M(X-Y)\right\|^{2}=0$, a contradiction to the assumption of $M$ satisfying the RIP of order 2. Therefore, $X=Y$ and $S_{+}(n, 1) \cap\left(\bigcap_{j=1}^{m} \mathcal{M}_{j}\right)$ is a singleton.

To prove convergence to $S_{+}(n, 1) \cap\left(\cap_{j=1}^{m} \mathcal{M}_{j}\right)$ of the sequence $\left(X^{k}\right)_{k \in \mathbb{N}}$ generated by

$$
X^{k+1}=P_{S_{+}(n, 1)} P\left(\bigcap_{j=1}^{m} \mathcal{M}_{j}\right)^{X^{k}} \quad(\forall k \in \mathbb{N})
$$

we define the following two auxiliary functions $g$ and $q$.

$$
\begin{aligned}
g(X) & :=\frac{1}{2} \operatorname{dist}\left(X,\left(\bigcap_{j=1}^{m} \mathcal{M}_{j}\right)\right)^{2}=\frac{1}{2}\left\|X-P_{\left(\bigcap_{j=1}^{m} \mathcal{M}_{j}\right)} X\right\|^{2}, \\
q(X, Y) & :=g(Y)+\left\langle X-Y, M^{\dagger}(M Y-b)\right\rangle+\frac{1}{2}\|X-Y\|^{2} .
\end{aligned}
$$

By the definition of the projector,

$$
X^{k+1} \in \operatorname{argmin}_{X \in S_{+}(n, 1)}\left\{q\left(X, X^{k}\right)\right\} .
$$

To see this, note the following

$$
\begin{aligned}
& q\left(X, X^{k}\right) \\
& =g\left(x^{k}\right)+\left\langle X-X^{k}, M^{\dagger}\left(M X^{k}-b\right)\right\rangle+\frac{1}{2}\left\|X-X^{k}\right\|^{2} \\
& =\frac{1}{2}\left\|X^{k}-P\left(\bigcap_{j=1}^{m} \mathcal{M}_{j}\right) X^{k}\right\|^{2}+\left\langle X-X^{k}, M^{\dagger}\left(M X^{k}-b\right)\right\rangle+\frac{1}{2}\left\|X-X^{k}\right\|^{2},
\end{aligned}
$$


by the definition of $g$. Then

$$
\begin{aligned}
& q\left(X, X^{k}\right) \\
& =\frac{1}{2}\left\|X^{k}-P\left(\bigcap_{j=1}^{m} \mathcal{M}_{j}\right) X^{k}\right\|^{2}+\left\langle X-X^{k}, M^{\dagger}\left(M X^{k}-b\right)\right\rangle+\frac{1}{2}\left\|X-X^{k}\right\|^{2} \\
& =\frac{1}{2}\left\|\left(X^{k}-P\left(\bigcap_{j=1}^{m} \mathcal{M}_{j}\right) X^{k}\right)-\left(X^{k}-X\right)\right\|^{2} \\
& =\frac{1}{2}\left\|\left(X-X^{k}+\left(X^{k}-P\left(\bigcap_{j=1}^{m} \mathcal{M}_{j}\right) X^{k}\right)\right)\right\|^{2} .
\end{aligned}
$$

Now, by the definition of the alternating projection sequence,

$$
\left.X^{k+1}=P_{S_{+}(n, 1)} P\left(\bigcap_{j=1}^{m} \mathcal{M}_{j}\right) X^{k}=P_{S_{+}(n, 1)}\left(X^{k}-\left(\operatorname{Id}-P\left(\bigcap_{j=1}^{m} \mathcal{M}_{j}\right)\right)\right) X^{k}\right),
$$

which together with (7.4), yields

$$
\begin{aligned}
X^{k+1} & \in \operatorname{argmin}_{X \in S_{+}(n, 1)}\left\{\| X-\left(X^{k}-\left(\operatorname{Id}-P\left(\cap_{j=1}^{m} \mathcal{M}_{j}\right) X^{k}\right) \|^{2}\right\}\right. \\
& =\operatorname{argmin}_{X \in S_{+}(n, 1)}\left\{q\left(X, X^{k}\right)\right\} .
\end{aligned}
$$

That is, $X^{k+1}$ is a minimizer of $q\left(X, X^{k}\right)$ in $S_{+}(n, 1)$. Furthermore, by the definition of the $g$ we have $g\left(X^{k+1}\right)=\frac{1}{2}\left\|M^{\dagger}\left(M X^{k+1}-b\right)\right\|^{2}$. Rewriting this term yields

$$
\begin{aligned}
& g\left(X^{k+1}\right) \\
& =\frac{1}{2}\left\|M^{\dagger}\left(M X^{k+1}-b\right)\right\|^{2} \\
& =\frac{1}{2}\left\|M^{\dagger} M\left(X^{k+1}-X^{k}\right)+M^{\dagger}\left(M X^{k}-b\right)\right\|^{2} \\
& =g\left(X^{k}\right)+\left\langle M^{\dagger} M\left(X^{k+1}-X^{k}\right), M^{\dagger}\left(M X^{k}-b\right)\right\rangle+\frac{1}{2}\left\|M^{\dagger} M\left(X^{k+1}-X^{k}\right)\right\|^{2} \\
& \leq f\left(X^{k}\right)+\left\langle M^{\dagger} M\left(X^{k+1}-X^{k}\right), M^{\dagger}\left(M X^{k}-b\right)\right\rangle+\frac{1}{2}\left\|\left(X^{k+1}-X^{k}\right)\right\|^{2},
\end{aligned}
$$


since $M$ satisfies the RIP of order 2. Then, by the definition of $M^{\dagger}$,

$$
\begin{aligned}
& g\left(X^{k+1}\right) \\
& \leq f\left(X^{k}\right)+\left\langle M^{\dagger} M\left(X^{k+1}-X^{k}\right), M^{\dagger}\left(M X^{k}-b\right)\right\rangle+\frac{1}{2}\left\|\left(X^{k+1}-X^{k}\right)\right\|^{2} \\
& \leq f\left(X^{k}\right)+\left\langle M^{*}\left(M M^{*}\right)^{-1} M\left(X^{k+1}-X^{k}\right), M^{*}\left(M M^{*}\right)^{-1}\left(M X^{k}-b\right)\right\rangle \\
&+\frac{1}{2}\left\|\left(X^{k+1}-X^{k}\right)\right\|^{2} \\
& \leq f\left(X^{k}\right)+\left\langle M M^{*}\left(M M^{*}\right)^{-1} M\left(X^{k+1}-X^{k}\right),\left(M M^{*}\right)^{-1}\left(M X^{k}-b\right)\right\rangle \\
&+\frac{1}{2}\left\|\left(X^{k+1}-X^{k}\right)\right\|^{2} \\
& \leq f\left(X^{k}\right)+\left\langle M\left(X^{k+1}-X^{k}\right),\left(M M^{*}\right)^{-1}\left(M X^{k}-b\right)\right\rangle+\frac{1}{2}\left\|\left(X^{k+1}-X^{k}\right)\right\|^{2} \\
& \leq f\left(X^{k}\right)+\left\langle X^{k+1}-X^{k}, M^{*}\left(M M^{*}\right)^{-1}\left(M X^{k}-b\right)\right\rangle+\frac{1}{2}\left\|\left(X^{k+1}-X^{k}\right)\right\|^{2} \\
& \leq f\left(X^{k}\right)+\left\langle X^{k+1}-X^{k}, M^{\dagger}\left(M X^{k}-b\right)\right\rangle+\frac{1}{2}\left\|\left(X^{k+1}-X^{k}\right)\right\|^{2} \\
&= q\left(X^{k+1}, X^{k}\right),
\end{aligned}
$$

implying that $g\left(X^{k+1}\right) \leq q\left(X^{k+1}, X^{k}\right)$. But since $X^{k+1}$ minimizes $q\left(X, X^{k+1}\right)$ over $S_{+}(n, 1)$, we know that for $\{\bar{X}\}=S_{+}(n, 1) \cap\left(\cap_{j=1}^{m} \mathcal{M}_{j}\right)$,

$$
q\left(X^{k+1}, X^{k}\right) \leq q\left(\bar{X}, X^{k}\right)
$$

Moreover, by the RIP assumption we have

$$
\begin{aligned}
q\left(\bar{X}, X^{k}\right) & =g\left(X^{k}\right)+\left\langle\bar{X}-X^{k}, M^{\dagger}\left(M X^{k}-b\right)\right\rangle+\frac{1}{2}\left\|\bar{X}-X^{k}\right\|^{2} \\
& \leq g\left(X^{k}\right)+\left\langle\bar{X}-X^{k}, M^{\dagger}\left(M X^{k}-b\right)\right\rangle+\frac{1}{2(1-\delta)}\left\|M^{\dagger} M\left(\bar{X}-X^{k}\right)\right\|^{2} \\
& =g\left(X^{k}\right)+\left\langle\bar{X}-X^{k}, M^{\dagger}\left(M X^{k}-b\right)\right\rangle+\frac{1}{2(1-\delta)}\left\|M^{\dagger}\left(b-M X^{k}\right)\right\|^{2} .
\end{aligned}
$$

Using the definition of $g$, we deduce

$$
\begin{aligned}
q\left(\bar{X}, X^{k}\right) & \leq\left(1+\frac{1}{2(1-\delta)}\right) g\left(X^{k}\right)+\left\langle\bar{X}-X^{k}, M^{\dagger}\left(M X^{k}-b\right)\right\rangle \\
& =\left(1+\frac{1}{2(1-\delta)}\right) g\left(X^{k}\right)+\left\langle M^{\dagger} M\left(\bar{X}-X^{k}\right), M^{\dagger}\left(M X^{k}-b\right)\right\rangle,
\end{aligned}
$$

where we used that $M^{\dagger} M=\mathrm{Id}$, since $M\left(M^{\dagger} M\right)=M\left(M^{*}\left(M M^{*}\right)^{-1} M\right)=M$. 
Utilizing again the definition of $g$, yields

$$
\begin{aligned}
q\left(\bar{X}, X^{k}\right) & \leq\left(1+\frac{1}{2(1-\delta)}\right) g\left(X^{k}\right)+\left\langle M^{\dagger} M\left(\bar{X}-X^{k}\right), M^{\dagger}\left(M X^{k}-b\right)\right\rangle \\
& =\left(1+\frac{1}{2(1-\delta)}\right) g\left(X^{k}\right)-\left\langle M^{\dagger}\left(M X^{k}-b\right), M^{\dagger}\left(M X^{k}-b\right)\right\rangle \\
& =\left(1+\frac{1}{2(1-\delta)}\right) g\left(X^{k}\right)-2 g\left(X^{k}\right) \\
& =\left(\frac{\delta}{1-\delta}\right) g\left(X^{k}\right)
\end{aligned}
$$

Combining our results, we deduce

$$
g\left(X^{k+1}\right) \leq q\left(X^{k+1}, X^{k}\right) \leq q\left(\bar{X}, X^{k}\right) \leq\left(\frac{\delta}{1-\delta}\right) g\left(X^{k}\right) .
$$

Since we assumed $\delta \in[0,1 / 2)$, we have $\frac{\delta}{1-\delta} \in[0,1)$, and thus $\operatorname{dist}\left(X^{k}, \bigcap_{j=1}^{m} \mathcal{M}_{j}\right) \rightarrow$ 0 linearly as $k \rightarrow \infty$, with rate bounded above by $\sqrt{\frac{\delta}{1-\delta}}<1$. Since the iterates $X^{k}$ all lie in $S_{+}(n, 1)$ by definition, this proves convergence to the intersection $S_{+}(n, 1) \cap\left(\bigcap_{j=1}^{m} \mathcal{M}_{j}\right)=\{\bar{X}\}$ as claimed.

Note that by the description of our problem in (7.3) and the choice of the initial matrix $X^{0}$ in Theorem 7.0.2 we are only considering symmetric matrices in the proof of Theorem 7.0.2. Therefore, it would be enough to assume a RIP for symmetric matrices only, instead for all matrices $X \in \mathbb{C}^{n \times n}$. Although such a restriction would be less demanding, we decided to formulate the RIP in Definition 7.0.1 for general $X \in \mathbb{C}^{n \times n}$. The conclusion of Theorem 7.0.2 is that, when certain RIP assumptions are satisfied, there exists a unique solution to the phase retrieval problem in matrix space. By the relationship between the matrix problem (7.3) and our original problem (7.2), this implies that there is a unique solution (up to a global phase) to the original problem. Note that, in this chapter, we study the phase retrieval problem with only measurement constraints. Also, one has to keep in mind that alternating projection onto the sets in (7.3) in the space $\mathbb{C}^{n \times n}$ is a different algorithm than alternating projections in the vector space $\mathbb{C}^{n}$. Thus the iterates themselves, in contrast to the limiting result, do not help us to make statements on the problem (7.2) in $\mathbb{C}^{n}$. Moreover, computing the restricted isometry constant in general is NP-hard [123]. Nevertheless, there is hope that one might compute a RIP constant $\delta$ with a desirable value. A number of studies have shown that Gaussian matrices satisfy the RIP with high probability (see for instance $[6,28]$ ). Moreover, there are deterministic studies dedicated to the construction of matrices satisfying the RIP (see $[34,81]$ ). What remains open is a proof that the matrix $M$ in Theorem 7.0.2 satisfies the RIP or, alternatively, under which conditions $M$ satisfies the RIP. 
Comparing the statements just made with our discussion in Chapter 6, we collect the following. Unique determination of the phase object is not a given fact for the phase retrieval problem. Assuming additional RIP conditions imply that there exists a unique solution to the phase retrieval problem (up to a global phase). This is still true when adding additional a priori assumptions. However, a connection between fixed points of the algorithms we discussed and the unique solution of the phase retrieval problem is missing. We believe that there is a connection between the fixed points of alternating projections (or any other suitable projection method) in $\mathbb{C}^{n \times n}$ and the fixed points of corresponding projection methods in $\mathbb{C}^{n}$. A different method analyzed was alternating projections in $\left(\mathbb{C}^{n \times n}\right)^{m}$ by Neumann in $[109$, Section 10.4]. His analysis can be as well interpreted as an alternating projection step between averaged projection method on the sets $\mathcal{M}_{1}, \mathcal{M}_{2}, \ldots, \mathcal{M}_{m}$ and the set $S_{+}(n, 1)$ in $\mathbb{C}^{n \times n}$. Thus, instead of using the projectors onto the intersection of the sets $\mathcal{M}_{i}$, the individual projectors onto the sets are used. Although, the connection between this approach and averaged projections in $\mathbb{C}^{n}$ applied to the sets $M_{1}, M_{2}, \ldots, M_{m}$ is not direct, we believe that a further investigation of their fixed points, respectively, yields a deeper insight into the relation between RIP conditions in $\mathbb{C}^{n \times n}$ and the fixed points of projection methods in $\mathbb{C}^{n}$. Thus, further research in this direction would approach the question when fixed points of projection methods are not only approximations but solutions to the phase retrieval problem. 


\section{CHAPTER 8}

\section{Conclusion And Future Work}

This thesis contributes to the existing literature on feasibility problems. In particular, we studied the relaxed Douglas-Rachford method and its cyclic version in detail to solve feasibility problems. We formulated a new regularity notion for nonconvex sets in the framework of existing regularity schemes, super-regularity at a distance, to prove local linear convergence of the relaxed Douglas-Rachford method. Relying on the regularity of the sets as well as the regularity of their intersection (we used the notion of subtransversality), we were able to quantify the rate of convergence and gave illustrative examples to create an intuition for the involved assumptions and constants. To complete our work, we highlighted special cases that satisfy either an individual assumption of our main convergence theorem (convexity and consistency) or fully satisfy all the assumptions (intersecting subspaces). To overcome the problem that the relaxed Douglas-Rachford method is only applicable in a 2-set feasibility instance, we focused on the cyclic relaxed Douglas-Rachford algorithm. Motivated by its good numerical performance, we first proved convergence result for closed and convex sets, and were able to characterize the fixed point set of the underlying operator explicitly for two sets. First results for more than two subspaces closed this chapter. In Chapter 6, we showed, how the phase retrieval problem fits into the framework of feasibility problems. Moreover, we gave an overview of commonly used projection methods to solve this problem and applied our results from the previous chapters to the phase retrieval problem. We illustrated our theoretical results with some numerical examples. The last chapter closed our survey with a discussions about solutions on the phase retrieval problem and the analyzed algorithms.

In the subsequent sections, we briefly state open questions in the respective topics and outline possible directions for further research. The section titles are loosely oriented towards the main chapters of this thesis. 


\subsection{Relaxed Douglas-RaChFord}

When analyzing the main result in Chapter 4 on local linear convergence, Theorem 4.4.2, there is one assumption that lacks an interpretation in the context of set-feasibility. This is the technical assumption in (4.20) given by

$$
\operatorname{dist}\left(\bar{\zeta}, \Psi_{g}(u)\right) \leq \sigma \operatorname{dist}\left(0, \Phi_{\bar{\zeta}}(u)\right)
$$

This assumption serves as a connection between subtransversality and metric subregularity as seen in Proposition 4.3.3. In the consistent setting, this assumption is always satisfied (see Proposition 4.3.4). In inconsistent cases, however, the technical assumption has to be shown for the individual problem instances. It is not known if there are more instances where this property is always satisfied. However, it is reasonable to assume that there is a trade-off relationship between the occurrence of the technical assumption and the regularity of the collection of sets. We conjecture that, if the regularity of the intersection and the sets themselves are strong enough, the technical assumption is redundant. This is in line with other results in the literature on alternating projections [92] and the Douglas-Rachford method [75,113]. Another direction for further research is the study of the relaxed Douglas-Rachford method on subspaces. In Section 4.7, we were able to state convergence results with rates dependent on the Friedrichs angle between the two subpaces, although our analysis was restricted to the setting with $A+B=\mathcal{E}$. Therefore, we can't cover simple constructions like two lines in $\mathbb{R}^{3}$. A generalization of our result without restricting the Minkowski sum to span the whole space would remove this drawback.

\subsection{Cyclic Relaxed Douglas-Rachford}

Our analysis of the cyclic relaxed Douglas-Rachford was limited to convex sets. A natural extension of our results are generalizations to nonconvex feasibility problems. For this, a study of the fixed points for more than two sets seems to be necessary, since one has to rely on their existence as well as the regularity of the cyclic relaxed Douglas-Rachford mapping to analyze its convergence behavior. This is more obvious when recalling the approach to show local linear convergence for the relaxed Douglas-Rachford method in Chapter 4. As mentioned in Section 5.3, we suggest starting the analysis of the fixed points for "easy" sets like subspaces (or convex sets), since in this case experiments show that the fixed points lie in certain areas like lines or convex hulls. 


\subsection{Phase RETRIEVAL}

As an application, this thesis dealt with the phase retrieval problem and different algorithms to solve it. Most of them can be identified with well-known projection methods. Thus, the focus might be not on inventing new algorithms, but rather to better understand when to choose a particular algorithm and how to set the involved relaxation parameters. As seen in Section 6.6, it appears that for the relaxed Douglas-Rachford method, a smooth change of the relaxation parameter speeds up the algorithm. This was already observed by Luke in [97]. The same need not be true for the cyclic relaxed Douglas-Rachford method, illustrating the unstable behavior with respect to the relaxation parameter. Further research in this direction explaining this behavior would assists the numerical analysis of the relaxed Douglas-Rachford method. Chapter 7 dealt with uniqueness in the phase retrieval problem. The assumption of the restricted isometry property in Theorem 7.0.2 is not verified for our problem instance yet. It therefore remains open whether the matrix $M$ in Theorem 7.0.2 satisfies the RIP or, alternatively, under which assumptions the matrix $M$ is satisfying the RIP. Furthermore, an investigation of the relationship between fixed points of projection methods in $\mathbb{C}^{n}$ and the fixed points of projection methods in $\mathbb{C}^{n \times n}$ is needed as discussed at the end of Chapter 7. 



\section{BIBLIOGRAPHY}

[1] J. P. Abrahams and A. G. W. Leslie, Methods used in the structure determination of bovine mitochondrial f1 atpase, Acta Crystallographica Section D: Biological Crystallography 52 (1996), no. 1, 30-42.

[2] N. Aronszajn, Theory of reproducing kernels, Transactions of the American Mathematical Society 68 (1950), no. 3, 337-404.

[3] F. J. A. Artacho, J. M. Borwein, and M. K. Tam, Douglas-Rachford feasibility methods for matrix completion problems, The ANZIAM Journal 55 (2014), no. 4, 299-326.

[4] _ Recent results on Douglas-Rachford methods for combinatorial optimization problems, Journal of Optimization Theory and Applications 163 (2014), no. 1, 1-30.

[5] D. Aze, A unified theory for metric regularity of multifunctions, Journal of Convex Analysis 13 (2006), no. 2, 225.

[6] B. Bah and J. Tanner, Improved bounds on restricted isometry constants for gaussian matrices, SIAM Journal on Matrix Analysis and Applications 31 (2010), no. 5, 2882-2898.

[7] J.-B. Baillon, P. L. Combettes, and R. Cominetti, There is no variational characterization of the cycles in the method of periodic projections, Journal of Functional Analysis 262 (2012), no. 1, 400-408.

[8] A. Bakan, F. Deutsch, and W. Li, Strong chip, normality, and linear regularity of convex sets, Transactions of the American Mathematical Society 357 (2005), no. 10, 3831-3863.

[9] A. Barty, S. Boutet, M. J. Bogan, S. Hau-Riege, S. Marchesini, K. SokolowskiTinten, N. Stojanovic, H. Ehrke, A. Cavalleri, S. Düsterer, et al., Ultrafast single-shot diffraction imaging of nanoscale dynamics, Nature Photonics 2 (2008), no. 7, 415.

[10] H. H. Bauschke, Projection algorithms and monotone operators, dissertation, Simon Fraser University, 1996. 
[11] H. H. Bauschke and J. M. Borwein, On the convergence of von Neumann's alternating projection algorithm for two sets, Set-Valued Analysis 1 (1993), no. 2, 185-212.

[12] _ Dykstra's alternating projection algorithm for two sets, Journal of Approximation Theory 79 (1994), no. 3, 418-443.

[13] H. H. Bauschke, J. M. Borwein, and A. S. Lewis, The method of cyclic projections for closed convex sets in Hilbert space, Contemporary Mathematics 204 (1997), 1-38.

[14] H. H. Bauschke, J. M. Borwein, and W. Li, Strong conical hull intersection property, bounded linear regularity, Jameson's property (g), and error bounds in convex optimization, Mathematical Programming 86 (1999), no. 1, 135160.

[15] H. H. Bauschke and P. L. Combettes, Convex analysis and monotone operator theory in Hilbert spaces, CMS Books Math./Ouvrages Math. SMC, Springer, New York, 2011.

[16] H. H. Bauschke, P. L. Combettes, and D. R. Luke, Phase retrieval, error reduction algorithm, and Fienup variants: a view from convex optimization, Optical Society of America 19 (2002), no. 7, 1334-45.

[17] _ Hybrid projection-reflection method for phase retrieval, Journal of the Optical Society of America A 20 (2003), no. 6, 1025-1034.

[18] _ Finding best approximation pairs relative to two closed convex sets in Hilbert spaces, Journal of Approximation Theory 127 (2004), no. 2, 178-192.

[19] H. H. Bauschke, J. Y. B. Cruz, Tran. T. A. Nghia, H. M. Phan, and X. Wang, The rate of linear convergence of the Douglas-Rachford algorithm for subspaces is the cosine of the friedrichs angle, Journal of Approximation Theory 185 (2014), 63-79.

[20] H. H. Bauschke, F. Deutsch, H. Hundal, and S.-H. Park, Accelerating the convergence of the method of alternating projections., Transactions of the American Mathematical Society 355 (2003), no. 9, 3433-3461.

[21] H. H. Bauschke and V. R. Koch, Projection methods: Swiss army knives for solving feasibility and best approximation problems with halfspaces, Infinite products of operators and their applications. A research workshop of the Israel Science Foundation, Haifa, Israel, May 21-24, 2012, vol. 636, Providence, RI: American Mathematical Society (AMS), 2015, pp. 1-40.

[22] H. H. Bauschke, D. R. Luke, H. M. Phan, and X. Wang, Restricted normal cones and the method of alternating projections: Applications, Set-Valued and Variational Analysis 21 (2013), no. 3, 475-501.

[23] _ Restricted normal cones and the method of alternating projections: theory, Set-Valued and Variational Analysis 21 (2013), no. 3, 431-473. 
[24] _ Restricted normal cones and sparsity optimization with affine constraints, Foundations of Computational Mathematics 14 (2014), no. 1, 63-83.

[25] H. H. Bauschke, D. Noll, and H. M. Phan, Linear and strong convergence of algorithms involving averaged nonexpansive operators, Journal of Mathematical Analysis and Applications 421 (2015), no. 1, 1-20.

[26] A. Beck and Y. Eldar, Sparsity constrained nonlinear optimization: Optimality conditions and algorithms, SIAM Journal on Optimization 23 (2013), no. 3, 1480-1509.

[27] A. Beck and M. Teboulle, A linearly convergent algorithm for solving a class of nonconvex/affine feasibility problems, Springer Optimization and Its Applications 49 (2011), 33-48.

[28] J. Blanchard, C. Cartis, and J. Tanner, Compressed sensing: How sharp is the restricted isometry property?, SIAM Review 53 (2011), no. 1, 105-125.

[29] J. Bolte, A. Daniilidis, O. Ley, and L. Mazet, Characterizations of łojasiewicz inequalities: subgradient flows, talweg, convexity, Transactions of the American Mathematical Society 362 (2010), no. 6, 3319-3363.

[30] J. Bolte, S. Sabach, and M. Teboulle, Proximal alternating linearized minimization or nonconvex and nonsmooth problems, Mathematical Programming 146 (2014), no. 1-2, 459-494.

[31] J. M. Borwein and D. R. Luke, Entropic regularization of the lo function, pp. 65-92, Springer New York, New York, NY, 2011.

[32] J. M. Borwein and M. K. Tam, A cyclic Douglas-Rachford iteration scheme, Journal of Optimization Theory and Applications 160 (2014), no. 1, 1-29.

[33] - The cyclic Douglas-Rachford method for inconsistent feasibility problems, Journal of Nonlinear and Convex Analysis 16 (2015), no. 4, 537-584.

[34] J. Bourgain, S. Dilworth, K. Ford, S. Konyagin, and D. Kutzarova, Explicit constructions of rip matrices and related problems, Duke Math. J. 159 (2011), no. $1,145-185$.

[35] F. E. Browder, Existence and approximation of solutions of nonlinear variational inequalities, Proceedings of the National Academy of Sciences 56 (1966), no. 4, 1080-1086.

[36] E. J. Candès, Y. C. Eldar, T. Strohmer, and V. Voroninski, Phase retrieval via matrix completion, SIAM Review 57 (2015), no. 2, 225-251.

[37] E. J. Candès and X. Li, Solving quadratic equations via phaselift when there are about as many equations as unknowns, Foundations of Computational Mathematics 14 (2014), no. 5, 1017-1026. 
[38] E. J. Candès, X. Li, and M. Soltanolkotabi, Phase retrieval from coded diffraction patterns, Applied and Computational Harmonic Analysis 39 (2015), no. 2, 277-299.

[39] E. J. Candès, T. Strohmer, and V. Voroninski, Phaselift: Exact and stable signal recovery from magnitude measurements via convex programming, Communications on Pure and Applied Mathematics 66 (2013), no. 8, 1241-1274.

[40] E. J. Candès and T. Tao, Decoding by linear programming, IEEE Transactions on Information Theory 51 (2005), no. 12, 4203-4215.

[41] E. J. Candès, M. B. Wakin, and S. P. Boyd, Enhancing sparsity by reweighted $\ell 1$ minimization, Journal of Fourier Analysis and Applications 14 (2008), no. 5, 877-905.

[42] E. J. Candès, X. Li, and M. Soltanolkotabi, Phase retrieval via Wirtinger flow: Theory and algorithms, IEEE Transactions on Information Theory $\mathbf{6 1}$ (2015), no. 4, 1985-2007.

[43] Y. Censor, W. Chen, P. L. Combettes, R. Davidi, and G. T. Herman, On the effectiveness of projection methods for convex feasibility problems with linear inequality constraints, Computational Optimization and Applications 51 (2012), no. 3, 1065-1088.

[44] H. N. Chapman, A. Barty, S.Marchesini, A. Noy, S. P. Hau-Riege, C. Cui, M. R. Howells, R. Rosen, H. He, J. C. H. Spence, U. Weierstall, T. Beetz, C. Jacobsen, and D. Shapiro, High-resolution ab initio three-dimensional $x$ ray diffraction microscopy, Journal of the Optical Society of America A 23 (2006), no. 5, 1179-1200.

[45] W. Cheney and A. A. Goldstein, Proximity maps for convex sets, Proceedings of the American Mathematical Society 10 (1959), no. 3, 448-450.

[46] C. K. Chui, F. Deutsch, and J. D. Ward, Constrained best approximation in Hilbert space, Constructive Approximation 6 (1990), no. 1, 35-64.

[47] P. L. Combettes and I. Yamada, Compositions and convex combinations of averaged nonexpansive operators., Journal of Mathematical Analysis and Applications 425 (2015), no. 1, 55-70.

[48] A. Daniilidis, D. R. Luke, and M. K. Tam, Characterizations of superregularity and its variants, arXiv: 1808.04978 (2018).

[49] M. N. Dao and H. M. Phan, Linear convergence of the generalized DouglasRachford algorithm for feasibility problems, Journal of Global Optimization 72 (2018), no. 3, 443-474.

[50] F. Deutsch, The angle between subspaces of a Hilbert space, Approximation theory, wavelets and applications, Kluwer Academic Publishers, Dordrecht, 1995, pp. 107-130. 
[51] _ Best approximation in inner product spaces, vol. 7, Springer Science \& Business Media, 2001.

[52] F. Deutsch and H. Hundal, The rate of convergence for the cyclic projections algorithm I: angles between convex sets, Journal of Approximation Theory 142 (2006), no. 1, 36-55.

[53] _ The rate of convergence for the cyclic projections algorithm II: norms of nonlinear operators, Journal of Approximation Theory 142 (2006), no. 1, $56-82$.

[54] _ The rate of convergence for the cyclic projections algorithm III: Regularity of convex sets, Journal of Approximation Theory 155 (2008), no. 2, $155-184$.

[55] A. L. Dontchev and R. T. Rockafellar, Implicit functions and solution mapppings, second ed., Springer-Verlag, Dordrecht, 2014.

[56] J. Douglas and H. H. Rachford, On the numerical solution of heat conduction problems in two and three space variables, Transactions of the American Mathematical Society 354 (2002), no. 1, 151-178.

[57] A. J. J. Drenth, A. M. J. Huiser, and H. A. Ferwerda, The problem of phase retrieval in light and electron microscopy of strong objects, Optica Acta: International Journal of Optics 22 (1975), no. 7, 615-628.

[58] D. Drusvyatskiy, C.-K. Li, D. C. Pelejo, Y.-L. Voronin, and H. Wolkowicz, Projection methods for quantum channel construction, Quantum Information Processing 14 (2015), no. 8, 3075-3096.

[59] V. Elser, Phase retrieval by iterated projections, Journal of the Optical Society of America A 20 (2003), no. 1, 40-55.

[60] V. Elser and I. Rankenburg, Deconstructing the energy landscape: Constraintbased algorithms for folding heteropolymers, Physical Review E 73 (2006), no. 2, 026702 .

[61] L. Elsner, I. Koltracht, and M. Neumann, Convergence of sequential and asynchronous nonlinear paracontractions, Numerische Mathematik 62 (1992), no. 1, 305-319.

[62] H. Federer, Curvature measures, Transactions of the American Mathematical Society 93 (1959), no. 3, 418-491.

[63] J. R. Fienup, Reconstruction of an object from the modulus of its fourier transform, Optics Letters 3 (1978), no. 1, 27-29.

[64] Space object imaging through the turbulent atmosphere, Optical Engineering 18 (1979), 529-534.

[65] _ Iterative method applied to image reconstruction and to computergenerated holograms, Optical Engineering 19 (1980), no. 3, 193297. 
[66] - Phase retrieval algorithms: a comparison, Applied Optics 21 (1982), no. $15,2758-2769$.

[67] J. R. Fienup and C. C. Wackerman, Phase-retrieval stagnation problems and solutions, Journal of the Optical Society of America A 3 (1986), no. 11, 1897-1907.

[68] K. Friedrichs, On certain inequalities and characteristic value problems for analytic functions and for functions of two variables, Transactions of the American Mathematical Society 41 (1937), no. 3, 321-364.

[69] R. W. Gerchberg and W. O. Saxton, A practical algorithm for the determination of phase from image and diffraction plane pictures, Optik 35 (1972), $237-246$.

[70] N. I. M. Gould, How good are projection methods for convex feasibility problems?, Computational optimization and applications 40 (2008), no. 1, 1-12.

[71] _ How good are extrapolated bi-projection methods for linear feasibility problems?, Computational optimization and applications 51 (2012), no. 3, 1089-1095.

[72] J. Hagemann, A-L. Robisch, D. R. Luke, C. Homann, T. Hohage, P. Cloetens, H. Suhonen, and T. Salditt, Reconstruction of wave front and object for inline holography from a set of detection planes, Optics Express 22 (2014), no. 10, 11552-11569.

[73] I. Halperin, The product of projection operators, 1962, pp. 96-99.

[74] R. Hesse and D. R. Luke, Nonconvex notions of regularity and convergence of fundamental algorithms for feasibility problems, SIAM Journal on Optimization 23 (2013), no. 4, 2397-2419.

[75] _ Nonconvex notions of regularity and convergence of fundamental algorithms for feasibility problems, SIAM Journal on Optimization 23 (2013), no. 4, 2397-2419.

[76] R. Hesse, D. R. Luke, and P. Neumann, Alternating projections and DouglasRachford for sparse affine feasibility, IEEE Transactions on Signal Processing 62 (2014), no. 18, 4868-4881.

[77] R. Hesse, D. R. Luke, S. Sabach, and M. K. Tam, Proximal heterogeneous block implicit-explicit method and application to blind ptychographic diffraction imaging, SIAM Journal on Imaging Sciences 8 (2015), no. 1, 426-457.

[78] A. D. Ioffe, Regularity on a fixed set, SIAM Journal on Optimization 21 (2011), no. 4, 1345-1370.

[79] _ Nonlinear regularity models, Mathematical Programming 139 (2013), no. 1-2, 223-242. 
[80] A. D. Ioffe and J. V. Outrata, On metric and calmness qualification conditions in subdifferential calculus, Set-Valued Analysis 16 (2008), no. 2-3, 199-227.

[81] M. A. Iwen, Simple deterministically constructible rip matrices with sublinear fourier sampling requirements, 2009 43rd Annual Conference on Information Sciences and Systems, March 2009, pp. 870-875.

[82] K. Jaganathan, Y. C. Eldar, and B. Hassibi, Phase retrieval: An overview of recent developments, arXiv:1510.07713 (2015).

[83] S. Kaczmarz, Angenäherte Auflösung von Systemen linearer Gleichungen., Bull. Int. Acad. Pol. Sic. Let., Cl. Sci. Math. Nat. A 35 (1937), 355-357.

[84] T. Kimura, Y. Joti, A. Shibuya, C. Song, S. Kim, K. Tono, M. Yabashi, M. Tamakoshi, T. Moriya, T. Oshima, et al., Imaging live cell in microliquid enclosure by $x$-ray laser diffraction, Nature communications 5 (2014), 3052 .

[85] M. Krenkel, M. Toepperwien, F. Alves, and T. Salditt, Three-dimensional single-cell imaging with $x$-ray waveguides in the holographic regime, Acta Crystallographica. Section A, Foundations of Crystallography 73 (2017), no. 4, 282-292.

[86] A. Y. Kruger, Error bounds and metric subregularity, Optimization 64 (2015), no. $1,49-79$.

[87] A. Y. Kruger, D. R. Luke, and N. H. Thao, About subtransversality of collections of sets, Set-Valued and Variational Analysis 25 (2017), no. 4, 701-729.

[88] _ Set regularities and feasibility problems, Mathematical Programming 168 (2018), no. 1-2, 279-311.

[89] M. Lai and J. Wang, An unconstrained $\ell_{q}$ minimization with $0<q \leq 1$ for sparse solution of underdetermined linear systems, SIAM Journal on Optimization 21 (2011), no. 1, 82-101.

[90] J. M. Lee, Smooth manifolds, 2 ed., Springer, 2003.

[91] A. Levi and H. Stark, Image restoration by the method of generalized projections with application to restoration from magnitude, Journal of the Optical Society of America A 1 (1984), no. 9, 932-943.

[92] A. S. Lewis, D. R. Luke, and J. Malick, Local linear convergence for alternating and averaged nonconvex projections, Foundations of Computational Mathematics 9 (2009), no. 4, 485-513.

[93] A. S. Lewis and J. Malick, Alternating projections on manifolds, Mathematics of Operations Research 33 (2008), no. 1, 216-234.

[94] G. Li and T. K. Pong, Douglas-rachford splitting for nonconvex optimization with application to nonconvex feasibility problems, Mathematical Pogramming 159 (2016), no. 1-2, 371-401. 
[95] X. Li and V. Voroninski, Sparse signal recovery from quadratic measurements via convex programming, SIAM Journal on Mathematical Analysis 45 (2013), no. 5, 3019-3033.

[96] P.-L. Lions and B. Mercier, Splitting algorithms for the sum of two nonlinear operators, SIAM Journal on Numerical Analysis 16 (1979), no. 6, 964-979.

[97] D. R. Luke, Relaxed averaged alternating reflections for diffraction imaging, Inverse problems 21 (2005), no. 1, 37.

[98] _ Finding best approximation pairs relative to a convex and proxregular set in a Hilbert space, SIAM Journal on Optimization 19 (2008), no. 2, 714-739.

[99] no. $1,1-5$.

[100] D. R. Luke, J. V. Burke, and R. G. Lyon, Optical wavefront reconstruction: Theory and numerical methods, SIAM Review 44 (2002), no. 2, 169.

[101] D. R. Luke and A-L. Martins, Convergence analysis of the relaxed DouglasRachford algorithm, arXiv:1811.11590 (2018).

[102] D. R. Luke, A-L. Martins, and M. K. Tam, Relaxed cyclic Douglas-Rachford algorithms for nonconvex optimization, ICML 2018 Workshop: Modern Trends in Nonconvex Optimization for Machine Learning, July 2018.

[103] D. R. Luke, S. Sabach, and M. Teboulle, Optimization on spheres: Models and proximal algorithms with computational performance comparisons, arXiv:1810.02893 (2018).

[104] D. R. Luke, M. Teboulle, and N. H. Thao, Necessary conditions for linear convergence of iterated expansive, set-valued mappings, Mathematical Programming (2018), 1-31.

[105] D. R. Luke, N. H. Thao, and M. K. Tam, Quantitative convergence analysis of iterated expansive, set-valued mappings, Mathematics of Operations Research 43 (2018), 1143-1176.

[106] S. Marchesini, A unified evaluation of iterative projection algorithms for phase retrieval, Review of Scientific Instruments 78 (2007), no. 1, 011301.

[107] B. S. Mordukhovich, Variational analysis and applications, Springer Monographs in Mathematics, Springer International Publishing, 2018.

[108] B. Natarajan, Sparse approximate solutions to linear systems, SIAM Journal on Computing 24 (1995), no. 2, 227-234.

[109] P. Neumann, Projection methods in sparse and low rank feasibility, dissertation, Georg-August-Universität Göttingen, 2015. 
[110] Z. Opial, Weak convergence of the sequence of successive approximations for nonexpansive mappings, Bulletin of the American Mathematical Society 73 (1967), no. 4, 591-597.

[111] J. M. Ortega and W. C. Rheinboldt, Iterative solution of nonlinear equations in several variables, Computer science and applied mathematics, Acad. Press, New York, 1970.

[112] J.-P. Penot, Metric regularity, openness and Lipschitzian behavior of multifunctions, Nonlinear Analysis: Theory, Methods \& Applications 13 (1989), no. $6,629-643$.

[113] H. Phan, Linear convergence of the Douglas-Rachford method for two closed sets, Optimization 65 (2016), no. 2, 369-385.

[114] G. Pierra, Decomposition through formalization in a product space, Mathematical Programming 28 (1984), no. 1, 96-115.

[115] R. A. Poliquin, R.T. Rockafellar, and L. Thibault, Local differentiability of distance functions, Transactions of the American Mathematical Society 352 (2000), no. 11, 5231-5249.

[116] Lord Rayleigh, XLVII. on the interference bands of approximately homogeneous light; in a letter to prof. a. michelson, The London, Edinburgh, and Dublin Philosophical Magazine and Journal of Science 34 (1892), no. 210, 407-411.

[117] B. Recht, M. Fazel, and P. Parrilo, Guaranteed minimum-rank solutions of linear matrix equations via nuclear norm minimization, SIAM Review 52 (2010), no. 3, 471-501.

[118] R. T. Rockafellar and R. J. Wets, Variational analysis, Springer, Berlin, 1998.

[119] D. A. Shapiro, Y.-S. Yu, T. Tyliszczak, J. Cabana, R. Celestre, W. Chao, K. Kaznatcheev, A. L. D. Kilcoyne, F. Maia, S. Marchesini, et al., Chemical composition mapping with nanometre resolution by soft x-ray microscopy, Nature Photonics 8 (2014), no. 10, 765.

[120] Y. Shechtman, Y. C. Eldar, O. Cohen, H. N. Chapman, J. Miao, and M. Segev, Phase retrieval with application to optical imaging: A contemporary overview, IEEE Signal Processing Magazine 32 (2015), no. 3, 87-109.

[121] B. F. Svaiter, On weak convergence of the Douglas-Rachford method, SIAM Journal on Control and Optimization 49 (2011), no. 1, 280-287.

[122] M. K. Tam, Regularity properties of non-negative sparsity sets, Journal of Mathematical Analysis and Applications 447 (2017), no. 2, 758 - 777. 
[123] A. M. Tillmann and M. E. Pfetsch, The computational complexity of the restricted isometry property, the nullspace property, and related concepts in compressed sensing, IEEE Transactions on Information Theory 60 (2014), no. 2, 1248-1259.

[124] J. Von Neumann, Functional operators, volume 2: The geometry of orthogonal spaces., vol. 2, Princeton University Press, 1951.

[125] I. Waldspurger, A. d'Aspremont, and S. Mallat, Phase recovery, maxcut and complex semidefinite programming, Mathematical Programming 149 (2015), no. $1,47-81$. 
$\epsilon$-subregularity, 23, 24, 26

alternating projections, 10

asymptotic regularity, 19

averaged, 17, 20

characterization, 18

composition, 18

pointwise almost averaged, 16

convergence

framework, 22

of composed mappings, 84

Q-linear convergence, 20

R-linear convergence, 20

convex set, 7

cyclic projections, 11

cyclic relaxed Douglas-Rachford, 13,

83

averaged, 85

convergence, 85

fixed points, 85, 88-90, 92

on subspaces, 91

on two closed convex sets, 86

difference vector, 50,55

distance function, 6

Douglas-Rachford, 11

error reduction, 102, 104

feasibility problem, 5

as minimization problem, 13

Fejér monotonicity, 19

fixed point set, 15

Friedrichs angle, 73, 76 hybrid input output method, 103, 105

inner iterations, 91

iterative algorithm, 10

linear regularity, 59, 72, 74

metric subregularity, 21, 22

nearest points, 87

nonexpansive, 17

pointwise almost nonexpansive, 16

normal cone, 9

limiting normal cone, 9

proximal normal cone, 9

optimization problem, 5

Phase Retrieval, 95

a priori constraint, 98

projection onto a priori

constraint, 99

prox-regular, 101

lifted, 116, 117

measurement constraint, 97

projection onto measurement constraint, 99

prox-regular, 101

problem, 96, 98

Pierra's product space trick, 12

polyhedrality, 22

projector, 6

affine set, 7

affine subspace, 9 
convex set, 7,18

linear subspace, 9

super-regular set at a distance, 28

translation formula, 8 prox-regularity, 101

reflector, 7 convex set, 8 relaxed Douglas-Rachford, 11, 41 firmly nonexpansive, 85 fixed points, 42, 45, 46, 48, 86 global linear convergence, 60 local linear convergence, 56 metric subregularity, 53

numerical analysis, 106

on phase retrieval, 103, 105

on subspaces, 72

pointwise almost nonexpansive, 37

subspaces, 76-78

restricted isometry property, 117

subtransversality, 31, 74

at common points, 59

super-regularity, 24

at a distance, 24-28, 36, 72

transversality, 74 


\section{Notation AND Symbols}

\begin{tabular}{|c|c|}
\hline $\mathbb{B}$ & open unit ball \\
\hline $\mathbb{B}_{\delta}(x)$ & open ball with radius $\delta$ centered around a point $x \in \mathcal{E}$ \\
\hline $\bar{\Omega}$ & closure of the set $\Omega$ \\
\hline $\mathbb{C}$ & set of complex numbers \\
\hline $\mathbb{C}^{n}$ & set of $n$-dimensional complex-valued vectors \\
\hline $\mathcal{O}$ & a priori constraint in the phase retrieval problem \\
\hline$A$ & amplitude constraint \\
\hline $\mathcal{A}_{s}$ & sparsity constraint \\
\hline $\mathfrak{S}$ & support constraint \\
\hline $\mathfrak{S}_{+}$ & support and non-negative constraint \\
\hline $\mathfrak{S}_{r}$ & support and real-valued constraint \\
\hline $\operatorname{dist}(x, y)$ & and $y$ \\
\hline $\operatorname{dist}(x, \Omega)$ & distance between the point $x$ and the set $\Omega$ \\
\hline $\operatorname{dist}\left(\Omega_{1}, \Omega_{2}\right)$ & distance between the sets $\Omega_{1}$ and $\Omega_{2}$ \\
\hline$T_{A, B}$ & the Douglas-Rachford mapping for the sets $A$ and $B$ \\
\hline$T_{A, B}^{\lambda}$ & $\begin{array}{l}\text { the relaxed Douglas-Rachford mapping for the sets } \\
A \text { and } B \text { and relaxation parameter } \lambda\end{array}$ \\
\hline$T_{[1 \ldots m]}^{\lambda}$ & $\begin{array}{l}\text { the cyclic relaxed Douglas-Rachford mapping for the } \\
\text { sets } \Omega_{1}, \Omega_{2}, \ldots, \Omega_{m} \text { and relaxation parameter } \lambda\end{array}$ \\
\hline $\mathcal{E}$ & real Euclidean space \\
\hline Fix $T$ & fixed point set of the mapping $T$ \\
\hline $\mathcal{F}$ & Fourier transform \\
\hline$f: D \rightarrow \Omega$ & single-valued mapping fr \\
\hline $\begin{array}{l}f: D \rightrightarrows \Omega \\
\operatorname{gph} T\end{array}$ & $\begin{array}{l}\text { set-valued mapping from the set } D \text { to the set } \Omega \\
\text { graph of the mapping } T\end{array}$ \\
\hline $\mathcal{H}$ & real \\
\hline$\langle\cdot, \cdot\rangle$ & oduct on Fuclide \\
\hline$M_{j}$ & $\begin{array}{l}j \text {-th measurement taken in the phase retrieval prob- } \\
\text { lem }\end{array}$ \\
\hline & Minkowski difference of the sets $\Omega_{1}$ and $\Omega_{2}$ \\
\hline$\Omega_{1}+\Omega_{2}$ & Minkowski sum of the sets $\Omega_{1}$ and $\Omega_{2}$ \\
\hline
\end{tabular}


$\mathbb{N} \quad$ set of natural numbers $\{0,1,2, \ldots\}$

$\|\cdot\| \quad$ norm induced by the inner product on Euclidean space

$\|\cdot\|_{0} \quad \ell_{0}$ norm

$N_{\Omega} \quad$ limiting (proximal) normal cone of $\Omega$ at $x$

$N_{\Omega}^{\text {prox }} \quad$ proximal normal cone of $\Omega$ at $x$

$U^{\perp} \quad$ orthogonal complement of the subspace $U$

$E \quad$ points in the set $A$ closest to the set $B$

$F \quad$ points in the set $B$ closest to the set $A$

$W(\bar{\zeta})$ affine transformation of the diagonal of the product space

$T_{\bar{\zeta}} \quad$ lifted relaxed Douglas-Rachford mapping

$\zeta \quad$ difference vector

$W_{0}(g) \quad$ set of fixed points of the mapping $P_{\Omega_{g}} \circ \Pi$

$\Omega_{g} \quad$ product of the collection of sets in convergence proof for $T_{A, B}^{\lambda}$

$\mathcal{Z}(x, g)$ set of difference vectors

$P_{\Omega} x \quad$ projector of the point $x$ with respect to the set $\Omega$

$\mathbb{R} \quad$ set of real numbers

$\mathbb{R}_{+} \quad$ set of nonnegative real numbers

$\mathbb{R}_{++} \quad$ set of positive real numbers

$\mathbb{R}^{n} \quad$ set of $n$-dimensional real-valued vectors

$\mathbb{R}_{+}^{n} \quad$ set of $n$-dimensional real-valued vectors with nonnegative entries

$\mathbb{R}_{++}^{n} \quad$ set of $n$-dimensional real-valued vectors with positive entries

$R_{\Omega} x \quad$ reflector of the point $x$ with respect to the set $\Omega$

$\mathbb{S} \quad$ unit sphere 